$$
38
$$




\section{Ditslar of Coleophare}

Department of Entonologs UB. National Museum 




\section{$Q L$}

bark H.Wrinarot

Entrikhis

Ameno

LIST

OF THE

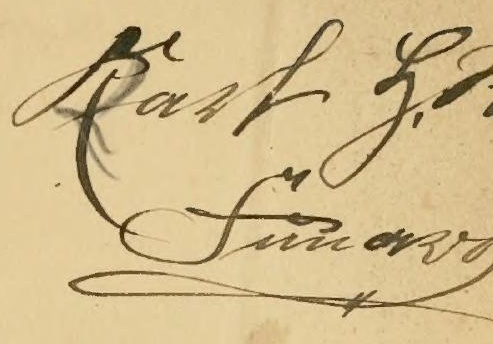

R.D. PERKINS

\section{COLEOPIERA}

America, Noxth of Litexico,

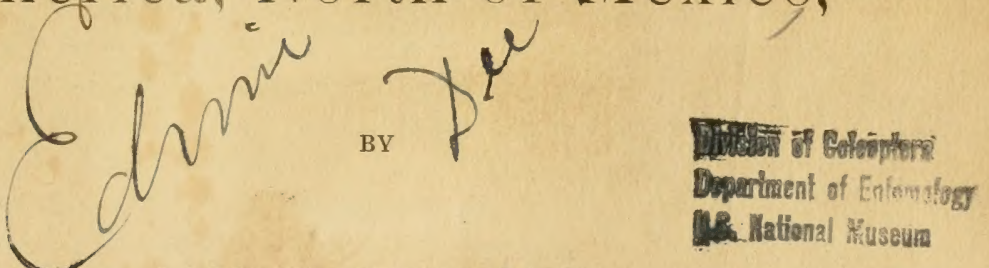

SAMUEL HENSHAW.

PHILADELPHIA:

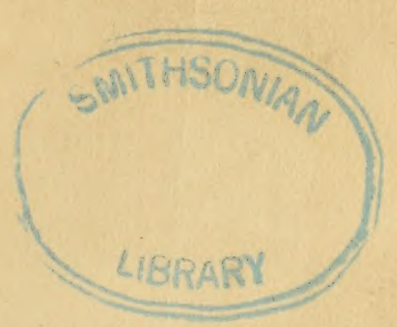

American Entomological Society.

I 885 . 
PRESS OF

“ GeO. B. CRESSON, PHILADELPHIA. 


\section{PREFACE.}

The twelve years which have passed since the publication of the Check List by Mr. G. R. Crotch have proved its usefulness to students of our fauna. By facilitating exchanges among collectors in different parts of our country it has made the diverse forms of our extensive fauna known in regions remote from their habitats.

In the present List the families and genera are arranged strictly in accordance with the Classification of the Coleoptera of North America by Drs. LeConte and Horn (Washington, 1883), the species following according to the most recent studies.

Synonyms are included only when necessary to connect the present with preceding lists. Species of uncertain value and position are added, unnumbered, at the end of the genera to which they are supposed to belong.

Genera inserted without specific names are known to occur here, but the species are undetermined.

Much of the synonomy in the present List is the result of a special study, chiefly by Dr. Horn, of the writings of the authors who have treated exclusively of the species of our fauna. In this manner the species of Beauvois (1805), Kirby (1837), Randall (1838), Newman (1838-42), Ziegler (1844-45), Bland (1862-64), Walker (1866) and Casey (1884-85) have been collated, and their value or synonomy determined frequently from the types themselves.

It is my intention to prepare for the pages of "Entomologica Americana" annual supplements. I shall, therefore, be indebted for information concerning any errors or omissions which may be noticed in the present List.

Owing to the length and the advantage of annotations the list of species erroneously accredited to our fauna will be printed elsewhere.

In conclusion I would acknowledge my indebtedness to Dr. G. H. Horn, whose kind interest and assistance have rendered the List more authoritative and valuable and very much lightened my work.

\section{SAMUEL HENSHAW.}

Boston, Sept. 15, 1885. 


\section{EXPLANATIONS:}

Synonyms are unnumbered.

Varieties are numbered with a letter added.

Pre-occupied names are marked with a $\|$.

Names cited erroneously are marked with a $\underset{\ddagger}{.}$

\section{CORRIGENDA.}

Page 9, No. 577, dele parallelus Mots.

“ 18, No. 1180, for Dej. read Say.

“ 18, No. 1201, for Mén. read Lec.

" 20, No. 1301, for Harr. read G. \& H.

< “ 21, No. 1384, for Lee. read Aubé.

ح "28, No. 1840, is $\|=$ Lecontei Schauff.

$\checkmark \quad$ " 28, No. $1853,=1854$.

“ "36, No. 2342, for scabrosus read seabiosus.

$\checkmark$ " 44, No. 2916, for Lec. read Mots.

$\checkmark$ " 44, No. 2940, for Matth. read G.\& $H$.

“ 45, No. $2975 a$ is printed $3975 a$.

$\checkmark$ " 45 , No. 2979 belongs in Bæocera.

“ 50, No. 3234, for Lec. read Lac.

$\checkmark$ " 55, No. 3486 is printed 3386 .

$\checkmark 59$, No. $3747,=$ pallidus Beauv.

$\checkmark$ “ 68 , under Elater dele semivittatus Say. $=$ No. 4483.

$\checkmark$ " 73, No. $4610,=$ aurulenta Linn.

$\checkmark$ “ 73, after No. 4610 insert $4610 \frac{1}{2}$ villosa Lec.

" 75, No. 4776, authority for typieum, for Lec. read Newm.

$\checkmark \quad$ “ 79 , No. 5023 is printed 5053 .

$\checkmark$ "81, authority for Trogodendron for Horn read Guér.

$\checkmark$ “. 100, insert No. 6242 nubjfer Jieg. , s

$\checkmark$ "105, authority for Donacia dentata should reád Hoppe.

$\checkmark$ “ 113, No. 7008, for Lec. read Melsh.

$\checkmark$ “ 113, No. 7053, for $\mathrm{Cr}$. read Melsh.

$\checkmark$ " 121, No. 7502, for Horn read Lap.

/0 $\checkmark$ "131, No. 8062 , for torta read torsa.

J " 134, No. 826\%, for Lac. read Lec. 


\section{Coleoptera of North America.}

\section{CICINDELIDAE.}

Amblychila Say.

1. cylindriformis Say.

Omus Esch.

2. Dejeani Reiche.

3. Edwardsii $\mathrm{Cr}$.

4. Audouini Reiche.

5. submetallicus Horn.

6. Hornii Lec.

7. californicus Esch.

8. sequoiarum $\mathrm{Cr}$.

9. ambiguus Schaupp.

10. Lecontei Horn.

11. lævis Horn.

Tetracha Hope.

- 12. carolina Linn.

- 13. virginica Linn.

Cicindela Linn.

14. Pilatei Guér.

14a. Belfragei Sallé.

C 15. celeripes Lec.

C 15a. cursitans Lec.

16. obsoleta Say.

C $\sigma \cdot 1$ ba. vulturina Lec.

16b. prasina Lec.

G/4 17. unipunctata Fab.

C 18. longilabris Say.

18a. perviridis Schaupp.

18b. Laurentii Schaupp.

18c. montana Lec.

19. scutellaris Say.

19a. unicolor Dej.
196. nigrior Schaupp.

19c. modesta Dej.

$19 d$. rugifrons $D e j$.

Cla 19e. Lecontei Hald.

20. nigrocærulea Lec.

C 21. pulchra Say.

22. Hornii Schaupp. anthracina || Horn.

2?. pimeriana Lec.

Ch 24. 6-guttata Fab.

C. 24a. violacea $F a b$.

Co 24b. patruela $D e j$.

2te. consentanea Dej.

Ch 25. purpurea Oliv.

Cb $25 \%$. Audubonii Lec.

25b. graminea Schaupp.

25c. cimarrona Lec.

25d. 10-notata Say.

Ch $25 e$. limbalis Lec.

$25 f$. limbalis $K l$.

$25 \%$. spreta Lec.

Ch 25h. amoena Lec.

C 25i. splendida Hentz.

Co 21. formosa Say.

c. 26a. generosa Dej.

26b. venusta $L e c$.

27. latesignata Lec.

27\%. tenuicincta Schaupp.

C 28. fulgida Say.

28a. Willistoni Lec.

29. senilis Horn.

30. hyperborea Lec.

30). limbata Say.

C. 31. ancocisconensis Harr.

et 32. vulgaris Say. 
C $32 \pi$. obliquata Kirby. $32 \mathrm{~b}$. vibex Horn.

Ch $3 \%$ repanda $D e j$.

C $3.3 a$ oregona Lec. 33b. guttifera Lec.

Ch isc. 12-guttata Dej.

(l) 34. pusilla Say. 34a. terricola $S a y$. $\therefore$ 4b. cyanella Lec.

(h 35. hirticollis Say.

C 36. cinctipennis Lec. :3ia. imperfecta Lec.

i7. lunalonga Schaupp.

sis. rectilatera Chd.

89. tenuisignata Lec.

Ch 40. punctulata $F a b$.

C tha. micans $F a b$.

C 41. tortuosa $D e j$. 41 u. serpens Lec. 41b. ascendens Lec. 41c. sigmoidea Lec.

C 12. dorsalis Say. 42a. media Lec. 42b. Saulcyi Guér.

4:. hamata Brullé.

44. marginata Fab.

C 4.) cuprascens Lec.

41. puritana Horn.

Ch 4h. macra Lec.

48. Wapleri Lec.
49. blanda $D e j$.

50. nevadica Lec.

51. sperata $L \varepsilon c$.

52. Gabbii Horn.

Ch 53. hirtilabris Lec. "

C 54. gratiosa Guér.

$\mathrm{Ch}$ 55. lepida Dej.

56. viridistictr Bates.

57. lemniseata Lec.

C 58. circumpicta Laf. ealifornica Mén.

58a. protextata Lec.

C 59. togata Laf.

60. pamphila Lec.

61. severa Laf.

62. striga Lec.

63. hæmorrhagica Lec.

6üa. pacifica Schaupp.

C 64. rufiventris $D e j$.

64a. cumatilis Lec.

64b. 16-punctata $K l$.

C 64c. Hentzii Dej.

65. marginipennis Dej.

66. Schauppii Horn.

67. abdominalis Fab.

67a. seabrosa Schaupp.

68. politula Lec.

69. Sommeri Mann.

bisignata Dokh.

\section{CARABID.}

Omophron Lat.

70. labiatum $F a b$.

C 71. nitidum Lec.

72. gilæ Lec,

73. obliteratum Horn.

74. robustum Horn.

75. dentatum Lec.

76. americanum Dej.

7\%. tessellatum Say.

7s. ovale Horn.

\section{Trachypachys Mots.}

79. inermis Mots.

80. Gibbsii Lec.

Cychrus Fab.

81. nitidicollis Chev. 81a. Brevoorti Lec.

82. stenostomus Web.

82a. Lecoutei Dej.

82b. bicarinatus Lec.

83. canadensis Chd.

84. relictus Horn.

85. regularis Lec.

86. elevatus $F a b$.

86a. unicolor Oliv.

87. viduus $D e j$.

87a. violaceus Lec.

88. Snowi Lec.

89. Guyoti Lec.

90. Ridingsii Bland.

91. Andrewsii Harr.

92. angusticollis Fisch.

93. cristatus Harr.

94. rugiceps Horn. 

95. cordatus Lec.
36. màrginatus Fisch.
96a. Fulleri Horn.
97. dissolutus Schaım.
98. interruptus Mén.
99. obliquus Lec.
100. striatus $L e c$.
101. ventricosus $D e j$.
102. mimus Horn.
103. punctatus Lec.
104. subtilis Schaum.
105. striatopunctatus Chd.
106. tuberculatus Harr.
107. Rickseckeri Lec.
108. Hemphillii Horn.
109. angulatus Harr.

\section{- Nomaretus Lec.}

110. bilobus Say.

111. fissicollis Lec.

112. cavicollis Lec.

113. imperfectus Horn.

114. debilis Lee.

\section{Carabus Linn.}

115. Vietinghovii Adams.

Ch 116. Maeander Fisch.

117. truncaticollis Fisch.

118. Chamissonis Fisch.

119. tædatus $F a b$.

119a. baccivorus Fisch.

119b. Agassii Lec.

119c. oregonensis Lec.

ch 120. sylvosus Say.

120a. finitimus Hald.

c 121. serratus Say.

ch 122. limbatus Say.

C 123. vinctus $W e b$.

123a. carinatus $D e j$.

123b. ligatus Germ.

__ ealifornicus Mots.

\section{Calosoma Web.}

Ch 124. externum Say.

125. macrum Lec.

126. protractum Lec.

$\mathrm{Ch}-127$. scrutator $\mathrm{Fab}$.

Ch 128. Willeoxi Lec.

Ch 129. frigidum Kirby.
C 130. Sayi Dej.

armatum Lap.

131. angulatum Chev.

132. prominens Lec.

133. peregrinator Guér. carbonatum Lec.

Ch 134. lugubre Lee.

135. Palmeri Horn.

136. triste Lec.

137. obsoletum Say.

138. Morrisoni Horn.

139. semilæve Lec.

140. simplex tec.

141. Haydeni Horn.

Cr 142. calidum Fab.

142a. tepidum Lec.

143. cancellatum Esch.

144. subæneum Chd.

145. moniliatum Le

146. discors Lec.

147. ilkesii Lec.

148. luxatum Say.

148a. striatulum Lec.

148b. Zimmermanni Lec.

149. latipenne Horn.

\section{Elaphrus Fab.}

150. Clairvillei Kirby.

151. lævigatus Lec.

152. olivaceus Lec.

153. cicatricosus Lec.

154. obliteratus Mann.

155. fuliginosus Say.

156. Lecontei $C r$.

157. riparius Linn.

158. viridis Horn.

159. pallipes Horn.

160. ruscarius Say.

\section{Diachila Mots.}

161. arctica Gyll.

162. subpolaris Lec.

\section{Blethisa Bon.}

163. Julii Lec.

164. quadricollis Hald.

165. multipunctata Linn.

166. oregonensis Lec. 


\section{Loricera Lat.}

167. crerulescens Linn.

168. californica Lec.

169. 10-punctata Esch.

170. foveata Lec.

171. congesta Mann.

Opisthius Kirby.

172. Richardsoni Kirby.

Notiophilus Dum.

17\%. æneus Hbst.

174. semiopacus Esch.

175. nitens Lec.

176. sylvaticus Esch.

177. semistriatus Say.

178. sibiricus Mots.

179. Hardyi Putz.

\section{Leistus Fröh.}

180. ferruginosus Mann.

181. piceus Frö̈h.

\section{Nebria Lat.}

182. ingens Horn.

183. ovipennis Lec.

184. diversa $L e c$.

185. virescens Horn.

186. purpurata Lec.

187. gregaria Fisch.

188. metallica Fisch.

189. Gebleri $D c j$.

190. viridis Horn.

191. obtusa Lec.

192. obliqua Lec. longula $L e c$.

193. suturalis Lec.

194. hudsonica Lec.

195. Sahlbergi Fisch.

196. bifaria Mann.

197. trifaria Lec.

198. Rathvoni Lec.

199. Mannerheimii Fisch.

200. Eschscholtzii Mén.

201. pallipes Say.

202. carbonaria Esch.

203. nivalis Payk.

__ elias Mots.

_- mollis Mrots.
Pelophila Dej.

204. Eschscholtzii Mann.

205. rudis Lec.

206. Ulkei Horn.

__ californica Mots.

\section{Metrius Esch.}

207. contractus Esch.

\section{Promecognathus Chd.}

208. lævissimus Dej. crassus Lee.

Pasimachus Bon.

-209. strenuus $L e c$.

210. sublævis Beauv.

211. marginatus $F a b$.

212. suksulcatus Say.

213. mexicanus Gray.

214. depressus Fab.

215. duplicatus Lee.

215a. costifer Lec.

216. obsoletus Lec.

217. elongatus Lec.

218. punetulatus Hald.

219. californicus Chd.

Scarites Fab.

-220. subterraneus Fab.

220a. substriatus Hald.

\section{Dyschirius Bon.}

221. tridentatus Lec.

222. patruelis Lec.

223. basalis Lec.

224. integer Lec.

225. nigripes Lec.

226. consobrinus Lec.

-227. gibbipennis Lec.

228. levifasciatus Horn.

229. Dejeanii Putz.

230. reneolus Lec.

231. longulus Lec.

232. globulosus Say.

233. hremorrhoidalis $D e j$.

234. terminatus Lec.

235. analis Lec.

236. brevispinus $L e c$. 
237. sphæricollis Say.

238. edentulus Putz.

239. truncatus Lec.

240. erythrocerus Lec.

241. marinus Lec.

242. obesus Lec.

243. sellatus Lec.

244. pallipennis Say.

245. salivagans Lec.

246. curvispinus Putz.

247. montanus Lec.

248. filiformis Lec.

249. sublevis Putz.

250. aratus Lec.

251. pumilus Dej.

252. abbreviatus Putz.

253. pilosus Lec.

254. hispidus Lec.

255. setosus $L e c$.

256. frigidus Mann.

257. transmarinus Mann.

\section{Clivina Lat.}

258. dentipes Dej.

259. impressifrons Lec.

260. texana Lec.

261. planicollis Lec.

262. punctulata Lec.

263. punctigera Lec.

264. rubicunda Lec.

265. pallida Say.

266. rufr LeC.

267. collaris Hbst.

268. analis Putz.

269. americana $D e j$.

270. morula Lec.

271. cordata Putz.

272. striatopunctata Dej.

273. ferrea Lec.

274. convexa Lec. '

275. bipustulata Fab.

276. marginipennis Putz.

277. postica Liec.

278. stigmula Putz.

279. sulcipennis $P u t z$.

\section{Aspidoglossa Putz.}

280. subangulata Chd.

\section{Schizogenius Putz.}

281. crenulatus Lec.

282. planulatus Lec.

283. lineolatus Say.

284. Sallei Putz.

285. ferrugineus Putz.

286. amphibius Hald.

287. depressus Lec.

288. pluripunctatus Lec.

Ardistomis Putz.

289. obliquata Putz.

290. Schaumii Lec.

291. morio Dej.

292. viridis Say.

293. puncticollis Putz.

\section{Panagæus Lat.}

294. Sallei Chd.

295. crucigerus Say.

296. fasciatus Say.

\section{Micrixys Lec.}

297. distinctus Hald.

\section{Pachyteles Perty.}

298. testaceus Horn.

299. parca Lec.

Nomius Lap.

300. pygmæus $D e j$.

Psydrus Lec.

301. piceus Lec.

Morio Lat.

302. monilicornis Lat.

Tachypus Még.

303. elongatus Mots.

\section{Bembidium Lat.}

Amerizus Chd.

Lymnceum Steph.

304. punctatostriatum Say.

305. carinula Chd.

impressum $\ddagger$ Lec.

306. Lorquinii Chd.

307. paludosum Sturm. 
307 c. lacustre $L e c$.

308. in rquale Say.

309. carinatum Lec.

310. sculpturatum Mots.

311. coxendix Say.

311a. nitidulum \| $D e j$.

312. Bowditchi Lec.

313. nitidum Kirby.

314. obliquulum Lec.

315. erasum Lec.

316. bifossulatum Lec.

317. americanum $D e j$.

318. dilatatum $L e c$.

319. antiquum $D e j$.

320. chalceum $D e j$.

321: concolor Kirby.

:322. longulum Lec.

323. quadrulum Lec.

324. recticolle Lec.

325. nigrum Say.

326. planatum Lec.

327. simplex Lec.

328. tetragonoderum $C h d$.

329. planiuseulum Mann.

330. complanulum Mann.

:31. incertum Mots.

332. tetraglyptum Mann.

333. funereum Lec.

334. Kuprianovi Mann.

335. biimpressum Mann.

3:iti. breve Mann.

337. 4-foveolatum Mann.

33.. Maeklini Lec.

3:3. nebraskense Lec.

340. fugax $L e c$.

341. planum Hald.

342. haplogonum Chd.

:44. transversale $D e j$. perspicuum Lec.

34t. erosum Mots.

iit5. lugubre Lec. stabile Lec.

itfi. parallelocolle Mots.

:47. striola Lec.

21. nevadense Ulke.

:49. bimaculatum Kirby.

:50. subinflatum 1 Lots.

351. lueidum Lec.

352. rupestre $D e j$.

352a. rupicola Kirby.
353. plagiatum Zimm.

354. lacunarium $Z \mathrm{imm}$.

355. tigrinum Lec.

356. mixtum Lec.

357. texanum Chd.

358. picipes Kirby. militare Casey.

359. scopulinum Kirby.

360. vinnulum Casey.

361. postremum Say.

362. aratum $L e c$.

363. Grapii Gyll. nitens Lec.

364. dyschirinum Lec.

365. insulatum $L \varepsilon c$.

366. cordatum Lec.

367. nubiculosum Chd.

368. hamiferum Chd.

369. laterimaculatum Mots.

370. undulatum Sturm.

371. incrematum Lec.

372. arcuatum Lec.

373. obtusangulum Lec.

374. approximatum Lec.

375. indistinctum $D e j$.

376. versutum Lec.

377. fraternum $L \epsilon c$.

378. viridicolle Laf.

379. Pilatei Chd.

380. variolosum Mots.

381. dorsale Say.

382. umbratum Lec.

383. sordidulum Chd.

384. conspersum Chd.

385. æneicolle Lec.

386. patruele $D e j$.

387. variegatum Say.

388. intermedium Kirby.

389. nigripes Kirby.

390. obscuromaculatum Mots.

391. versicolor Lec.

392. pictum Lec.

393. rubiginosum Lec.

394. constrictum Lec. acticola Casey. vernula Casey.

395. contractum Say. vividum Casey.

396. ephippiger Lec.

397. dejectum Casey. 
398. morulum Lec.

399. grandicolle Lec.

4(10. vile $L e c$.

401. rufotinctum Chd.

402. platyderum Chd.

403. Scudderi Lec.

404. sexpunctatum Lec.

405. suleatum Lec.

406. fortestriatum Mots.

407. affine Say.

408. dubitans Lec.

409. crurale Lec.

410. anguliferum Lec.

411. acutifrons Lec.

412. connivens Lec.

413. cautum Lec.

414. assimile Gyll.

415. mundum Lec.

416. mutatum $G$. $H$.

417. quadrimaculatum Linn.

418. præcinetum Lec.

419. pedicellatum Lec.

42ก. semistriatum Hald.

421. lævigatum Say.

422. trechiforme Lec.

423. iridescens Lec.

424. glabriusculum Mann.

425. spectabile Mann.

426. oblongulum Mann.

427. laticeps Lec.

Anillus Duval.

428. debilis Lec.

429. fortis Horn.

430. Dohrni Ehlers.

Tachys Schaum.

431. vittiger $L e c$.

432. mordax Lec.

433. proximus $S a y$.

434. virgo Lec.

435. scitulus Lec.

436. vorax Lec.

437. umbripennis $C h d$.

438. pumilus $D e j$.

439. sequax Lec.

440. corruscus Lec.

441. corax Lec.

442. edax Lec.

443. albipes $L e c$.
444. ventricosus $L e c$.

445. lævus Say.

446. misellus Laf.

447. pallidus Chd.

litoralis Casey.

448. occultator Casey.

449. nanus Gyll.

450. flavicauda Say.

451. nigriceps $D e j$.

452. ænescens Lcc.

453. tripunctatus Say.

454. vivax $L e c$.

455. capax Lec.

456. xanthopus $D e j$.

457. anthrax Lec.

458. obesulus Lec.

459. ferrugineus $D e j$.

460. truncorum Hald.

461. incurvus Sizy.

462. granarius $D e j$. glossema Casey.

463. nebulnsus Chd.

464. gemellus Casey.

465. fuscicornis Chd.

466. dolosus Lec.

467. audax Lee.

468. rapax Lec.

-- brunnicollis Mots.

_- glabrellus Mots.

_- marginicollis Mots.

__ reflexicollis Mots.

_- testaceolimbatus Mots.

\section{Pericompsus Lec.}

469. sellatus Lec.

470. ephippiatus Say.

471. lætulus Lec.

\section{Patrobus Dej.}

472. longicornis Say.

473. septentrionis $D e j$.

474. rugicollis Rand.

475. aterrimus $D e j$.

476. ealifornicus Mots.

477. angusticollis Mann.

478. fulvus Mann.

Pogonus Dej.

479. texanus Chd.

480. planatus Horn.

481. Lecontei Horn. 
Trechus Clairv.

482. rubens $F a b$.

483. chalybeus Mann.

484. ovjpennis Mots.

485. hydropicus Horn.

\section{Anophthalmus Sturm.}

486. Tellkampfii Er.

487. interstitialis Hubbard. ? striatus Mots.

488. Menetriesii Mots.

? ventricosus Mots.

489. tenuis Horn.

490. pusio Horn.

491. eremita Horn:

492. pubescens Horn.

493. audax Horn.

\section{Myas Dej.}

494. coracinus Say.

495. cyanescens Dej.

\section{Pterostichus Bon.}

Holciophorus Lec.

Lophoglossus Lec.

Piesmus Lec.

496. ater $D e j$.

497. serripes Lec.

498. morionides Chd.

499. tarsalis Lec.

500. crenicollis Lec.

501. planctus Lec.

502. herculeanus Mann.

503. validus $D e j$.

504. protractus Lec. parallelus Mrots.

505. vicinus Mann.

506. longulus Lec.

507. angustus $D e j$.

508. amethystinus $D e j$.

509. castaneus $(D e j$.

510. scutellaris Lec.

511. californicus $D e j$.

512. Menetriesii LITots.

513. castanipes Mén.

514. Spraguei Lec.

515. gracilior Lec. longicolle || Mots.

516. Hornii Lec.
517. Isabellæ Lec.

518. congestus MIén.

519. adoxus Say. zephyrus Cascy.

520. longicollis $L e c$.

521. sphodrinus Lec.

522. grandiceps Chd.

523. rostratus Newm.

524. substriatus Lec.

525. constrictus Suy.

526. incisus Lec.

527. vinctus $L e c$.

52s. unicolor Say.

529. rotundatus Lec.

530. spoliatus Newm.

5:1. acutus Lec.

5:32. obsoletus Say.

53:). lævipennis Lec.

5i:4. approximatus $L e c$.

5:35. morio Dej.

5:3i. faber Germ.

i:3. mancus Lec.

5.5. diligendus Chd. osculans Casey.

3i). honestus Say.

540. Lubricus Lec.

541. tumescens Lec.

542. obscurus Say.

543. ventralis Say.

544. lachrymosus Newm.

54. coracinus Newom. monedula Newm.

54li. stygicus Say.

547. relietus Newom.

54.. superciliosus Say.

C 54!?. moestus Say.

550. punctatissimus Rand.

.51. agonus Horn.

5.i2. fallax Dej.

55:. sculptus Lec.

5.5 1. obesulus Lec.

5ii. permundus Say.

5.51. Hamiltoni Horn.

5.7. subcordatus Lec.

5.5. scitulus Lcc.

5.i1. lretulus Lec.

itil. occidentalis Dei.

itil. corvus Lec.

51i2. cyaneus Lec.

56ii. texanus $L e c$. 
504. Sayi Brullé.

565. lucublandus $S a y$. manhattanis Casey.

iti6. bicolor Lec.

507. convexicollis Say.

j6i. ebeninus $D e j$.

369. caudicalis Say.

570. luctuosus Dej.

571. corvinus $D e j$.

572. Haldemani Lec.

57.) tartaricus Suy. , stremuus Lec.

574. serutator Lec.

575. gravis Lec.

576. submarginatus Say.

5it. purpuratus Lec. parallelus $M$ Tots.

57.. mutus Say. carbonaria Dej.

.79. lustrans Lec. puncticollis Mots.

581). Dennsylvanicus Lec.

jAl. vitreus $D e j$.

jस:2. orinomum Leach.

5...). Luczotii Dej.

ivit. oregonus $D e j$.

585. erythropus $D e j$.

586. splendidulus Lec.

587. patruelis Dej. linearis Mann.

iss. femoralis Kirby.

jificu. desidiosus Lec.

5xy. corrusculus tec.

j90. vindicatus Mann.

.j) ventricosus Esch.

5!2. subexaratus Manm.

59:3. pinguedineus Esch.

594. hyperboreus Mann.

595. hudsonicus Lec.

5!)6. similis Mén.

597. fatuus Mann.

.59. surgens Lec.

599. riparins Dej.

600. confusus Mann.

f01. subcaudatus M Lann.

602. empetricola Dej.

602a. frigidus Dej.

1003. mandibularis Kirby. brevicorne Kirby.

(i) 4. arcticola $C h$ l.
605. diplogma Chd.

606. labradorensis Chd.

607. subsinuosus Chd.

608. praetermissus $C h d$.

609. sejungenda $C h d$.

-... basilaris Mann.

- commixta Chd.

- commutabilis Mots.

-_ curtipenne Mots.

_ femoratus Mots.

-... iris Mots.

- - licinoides Mots.

- nimius Mots.

_... perseverus Mots.

- rotundicollis Mann.

_- ruficarpus Mann.

_- ruficollis Mann.

--_ rugulosus Mots.

-... texanus Mots.

Evarthrus Lec.

610. seximpressus Lec.

611. sigillatus $S a y$.

612. americanus $D e j$.

613. orbatus Newm.

614. Engelmanni Lec.

fi15. nonnitens Lec.

616. gravidus Hald.

617. heros Say.

6il8. torvus Lec.

619. colossus Lec.

620. sodalis Lec.

621. furtivus Lec.

622. Sallei Lec.

- acuminatus Chel.

imara Bon.

(i2:) avida Say.

iid. arenaria Lec.

1i25. similis $K i v b y$.

i2ti. rufimanus Kirby. reflexus Putz.

(i)7. eylindrica Lec.

(i2). jacobinæ Lec.

(i29. laticollis Lec.

630. carinata Lec.

i31. stupida Lec.

1332. Eschscholtzi Chd.

633. infausta Lee. 
6:i4. melanogustrica $D e j$.

635. hyperborea $D e j$.

6:66. brunnipennis Dej.

6:)7. elongata Lec.

63\%. glacialis Mann.

69.9. canadensis Putz.

Bit). tristis Putz.

i.11. Holmbergi Putz.

i) 12. somnolenta Putz.

843. adstrietus Putz.

6) 44. transversicollis Putz.

6.45. fulvipes Putz.

biti. exarata $D e j$.

f.47. latior Kirby. lrevistriatus Putz.

fit: septentrionalis Lec.

i)!). Putzeysii Horn.

6i50. apricarius Payk.

B51. angustata Say.

652. pallipes Kirby.

65.?. scitula Zimm.

654. longula Zimm.

bis. insignis $D e j$.

6.56. insularis Horn.

655. impuncticollis Say.

6.j. littoralis Mann.

fi.9. basillaris Say. marylandica Casey.

bit). crassispina Lec.

661. cupreolata Putz.

fei2. conflata Lec.

tit3. brunnipes Mots.

fitit. fallax Lec.

6i65. subpunctata Lec.

66ti. confusa Lec,

bi:. protensa Putz.

tiris. polita Lec.

1569. erratica Sturm.

i71). interstitialis Dej.

iiil. fareta $L e c$.

i:-2. ealifornica Dej.

i) $i \cdots$ fortis $L e c$.

bit. obesa Suy.

bi... diffinis Lec.

6iit. terrestris Lec.

6ii. chalcea Dej.

fi-: remotestriata Dej. indistineta Mfann. relucens Mann. apachensis Casey.
679. brunnea Gyll.

680. gibba Lec.

681. rubrica Hald. ferruginea Casey. pallida Casey.

682. subrenea Lec.

683. musculus Say.

684. harpalina Lee.

685. rectangula Lec.

686. aurata $D e j$.

887. acutangula Putz.

688. texana Putz.

Stolonis Mots.

689. Ulkei Horn.

Loxandrus Lec.

690. reflexus Lec.

691. saphyrinus $C h d$.

692. calathinus Lec.

693. floridanus Lec.

694. rectus Say.

694a. laticollis Chd.

695. lucens Chd.

696. parvulus $C h d$.

697. micans Chd.

698. brevicollis Lee.

699. minor Chd.

700. erraticus Dej.

701. celer $D e j$.

702. agilis $D e j$.

703. piceolus Chd.

704. crenulatus Chd.

705. proximus Chd.

706. rapidus $C h d$.

707. velox Dej.

707a. tæniatus Lec.

707b. piciventris Lec:

$707 c$. pusillus $L e c$.

708. rectangulus Lec.

709. erenatus Lec.

\section{Diplochila Brullé.}

710. laticollis Lec.

710a. major Lec.

711. impressicollis $D e j$.

712. obtusa Lec.

Dicælus Bon.

713. Iævipennis Lec. 
714. costatus LeC.

715. dilatatus Say.

716. planicollis Lec.

717. splendidus Srry.

718. purpuratus $B$ on.

719. quadratus Lec.

720. carinatus $D e j$.

721. alternans $D e j$.

722. sculptilis Say.

723. furvus $D e j$.

724. crenatus Lec.

725. ovalis Lec.

726. elongatus $B o n$. simplex $D e j$.

727. ambiguus Laf. opacus Laf. reflexus Lec. turbulentus Lec.

728. teter Bon.

729. politus $D e j$.

\section{Licinus Lat.}

730. silphoides $F a b$.

\section{Badister Clairv.}

731. notatus Hald.

732. pulchellus Lec.

733. bipustulatus Fab.

734. maculatus Lec.

735. elegans Lec.

736. obtusus Lec.

737. ferrugineus $D e j$.

73\%. anthracinus Lec.

7:39. Havipes Lec.

740. micans Lec.

741. reflexus Lec.

\section{Calathus Bon.}

742. gregarius Say.

743. ingratus $D e j$.

744. opaculus Lec.

745. quadricollis Lec.

746. Behrensii Mann.

747. obscurus Lec.

748. ruficollis $D e j$.

749. advena Lec.

750. impunctatus Say.

751. dubius Lec.

\section{Pristonychus Dej.}

752. complanatus $D e$.

753. terricola Hb.st.

Platynus Bon.

754. larvalis Lec.

755. caudatus Lec.

756. dissectus Lec.

757. jejunus $L e c$.

758. nivalis Horn.

759. hypolithus Say.

760. angustatus Dej.

761. agilis $L e c$.

762. quadrimaculatus Horn.

7ijs. maurus Mots.

76t. ovipennis Mann.

- 765. decens Say.

766. sinuatus $D e j$. depressus Huld.

767. opaculus Lec.

76is. tenuicollis Ler.

769. cincticollis Say.

770. reflexus Lec.

771. brunneomarginatus Mann. bicolor \| Lec.

772. extensicollis Say.

$772 a$. obscuratus $C h d$.

$772 b$, viridis $L e c$.

772c. cyanescens Mots.

772d. simplex Lee.

773. decorus Say.

774. californicus $D e j$.

$774 \pi$. floridanus Lec.

774b. texanus Lec.

775. bicolor $D e j$.

776. piceolus Lec.

77\%. anchomenoides Rand.

77.. ferruginosus $D e j$.

779. erasus Lec.

780. æeneolus Lec.

Til. quadratus Lec.

752. funebris $L e c$.

78:3. clemens Lec.

7.4. pusillus Lec.

7 K... suleipennis Horn.

7i6. errans Say. subcordatus Lec.

Tii. moerens $D e j$. 
7sk. tenuis Lec.

7ะ9. collaris Say.

7!). atratus Lec.

791. melanarius Dej.

792. propinquus G. \& $H$.

792. fraterculus $\mathrm{Lec}$.

794. affinis Kirby.

795. carbo Lec.

796. corvus Lec.

797. frater Iec. brevicollis Dej.

798. metallescens Lec.

799. deceptivus Lee.

Si)(1. eupripennis Say.

si)(ia. subsericeus Lec.

sil1. Hardyi Lee.

402. excavatus Dej.

80.) ferreus Hald.

sil.4. basalis Lec.

505. nutans Say.

G06. striatopunctatus $D e j$.

(i)7. albicrus $D e j$.

4i)s. picticornis Newm.

8119. maculicollis Dej.

i10. variolatus Lec.

\$11. sulcatus $D i j$.

412. striatus Dej.

81:. perforatus Lec.

814. octopunctatus Fab.

415. placidus Say.

ili. fossiger $D c j$.

\$17. deplanatus Mén.

418. cupreus $D c j$.

819. crassicollis Lee.

20. vicinus $G$. \& $H$.

821. obsoletus Say.

? Bogemanni Gyll.

?... quadripunctatus $D \in G$.

?.: bembidioides $K i r b !$

-2. xruginosus Dej.

-2. limbatus Say.

2i. crenistriatus Lec.

2.). rubripes Zimm.

-2. punctiformis Say.

-2!). sordens Kirby.

- il. picicornis Lcc. ? similis Kirby.

-:1. rufienrnis Lece.

-i:2. retractus Lee.

- :i. gemellus Lcc.
834. picipennis Kirby.

835. lutulentus Lec.

436. nigriceps $t \in c$.

837. maculifrons $S a y$.

8:3. exaratus MIann.

_- interstitialis Mots.

__ acuticollis Mots.

___ iridipennis Mots.

\section{Olisthopus Dej.}

839. parmatus Say.

840. micans Lec.

Perigona Iap.

Trechicus Lee.

841. nigriceps $D e j$.

Lachnophorus Dej.

842. elegantulus Mann.

\section{Euphorticus Horn.}

843. pubescens Dej.

Anchonoderus Reiche.

844. quadrinotatus Horn.

Atranus Lec.

845. pubescens Dej.

Leptotrachelus Lat.

846. dorsalis $F a b$.

S46a. pallidulus Mots.

Casnonia Lat.

S47. pennsylvanica Linn.

\$47a. suturnlis Chd.

84s. ludoviciana Sallé.

\section{Galerita Fab.}

849. atripes Lec.

850. decipiens Horn.

$\checkmark$ 851. janus Fab.

852. Lecontei Dej.

S53. bicolor Drury.

\section{Zuphium Lat.}

354. longicolle Lec. 
\$55. americanum Dej.

8.56. mexicanum Chd.

Diaphorus Dej.

857. Lecontei Dej.

858. tenuicollis Lee. tenuicornis Chd.

\section{Thalpius Lec.}

859. pygmaeus $D e j$.

8(i). Hornii Chd.

861. dorsalis Brullé.

81i2. rufulus Ler.

\section{Ega Lap.}

86.3. Sallei Chev.

864. laetula Lee.

\section{Tetragonoderus Dej.}

865. intersectus Germ.

866. fasciatus Hald. undulatus $L_{\mathrm{ecc}}$

867. latipennis Lec.

868. pallidus Horn.

Nemotarsus Lec.

869. elegans Lec.

\section{Lebia Lat.}

Aphelogenia Chd.

Dianchomena Chd.

Loxopeza Chd.

870. punctifera Lec.

871. divisa Lec.

-872. grandis Hentz.

873. atriventris Say.

874. tricolor Say. testacea Lec.

875. majuscula Chd.

876. atriceps Lec.

477. bitæniata Chev.

*78. pulchella Dej.

579. vivida Bates.

880. cyanipennis Dej.

s80a. ruficollis Lec.

881. marginicollis $D e j$.

881a. cupripennis Chd.

882. viridis $S a y$.

882a. moesta Lec. 882b. rhodopus $S z$.

883. pumila Dej.

884. pleuritica Lec.

885. lecta Horn.

886. viridipennis $D e j$.

887. montana Horn.

m-888. Iobulata Lec.

889. ornata $\$ a y$.

$889 \alpha$, axillaris $D e j$.

890. collaris Dej.

891. scapula Horn.

892. analis $D e j$.

893. fuscata $D e$ ). frigida Chd.

S94. abdominalis chd.

895. scapularis $D e j$.

896. miranda Horn.

\$97. furcata Lec.

898. depicta Horn.

899. vittata $F a b$.

$899 a$. Bpraguei Horn.

900. pectita Horn.

901. guttula Lec.

902. bivittata $F a b$.

903. bilineata Mots.

_- sublimbata Mots.

Coptodera Dej.

904. ærata Dej.

Phlœoxena Chd.

905. signata Dej.

Dromius Bon.

-906. piceus Dej.

906a. quadricollis Lec.

907. atriceps Lec.

Apristus Chd.

908. cordicollis Lec.

909. subsulcatus Dej. latens Lec.

910. laticollis Lec.

Blechrus Mots.

911. nigrinus Mann. linearis Ler.

912. lucidus Lec.

913. pusio Lec. 
Metabletus Sch.-Goeb.

914. americanus $D c j$. borealis Zimm.

\section{Axinopalpus Lec.}

915. biplagiatus $D e j$.

916. fusciceps $L c c$. nigriceps $L e c$.

\section{Tecnophilus Chd.}

Philotecnus Lec.

917. croceicollis MVén. chloridipennis Mots.

917 . Pilatei Chd.

917b. ruficollis Lec.

917c. nigricollis $L c c$.

\section{Euproctus Sol.}

918. trivittatus Lee.

\section{Callida Dej.}

919. platynoides Horn.

920. planulata Lec.

921. viridipennis Say.

922. decura $F a b$. cyanoptera Lec.

923. punctata Lec.

924. fulgida " $D e j$.

925. purpurea Say.

\section{Philophuga Mots.}

Glycia in spec.

926. viridicollis Lee.

927. amoena Lcc.

Hornii Chd.

928. viridis Dej. cyanea Mots.

929. castanea Horn.

\section{Plochionus Dej.}

930. pallens $F a b$.

931. timidus Hald.

932. amandus Nerom.

933. dorsalis Horn.

\section{Pinacodera Schaum.}

934. limbata Dej. levigata Mots.
934 2 . fuscata $D e ;$.

935. platicollis Say.

936. punctigera $L e c$.

937. semisulcata Horn.

938. sulcipennis Horn.

\section{Cymindis Lat.}

939. laticollis Say.

940. eribricollis $D e j$. abstrusa $L c c$.

941. planipennis $L c c$. brevipennis Zimm.

942. elegans Lec.

943. unicolor Kirby.

9.44. americana $D C j$. venator $D e j$.

945. cribrata Lec.

$\checkmark$ 946. pilosa Say.

947. borealis $L c c$.

948. neglecta Hald.

\section{Apenes Lec.}

949. lucidula $D c_{j}$.

$949 a$. angustata $\mathrm{Sz}$.

950. sinuata Say.

951. opaca Lec.

952. nebulosa $L e c$.

\section{Eucaerus Lec.}

953. varieornis Lee.

Pentagonica Schm.-Goeb. Rhombodera Reiche.

954. flavipes Lcc. bicolor Lec. pallipes $L e c$.

_._ angulata $B$ oh.

Onota Chd.

955. floridana Horn.

\section{Helluomorpha Lap.}

956. nigripennis $D e j$.

957. Clairvillei $D e j$.

958. præusta Dej.

959. bicolor Harr. laticornis Dej.

960. texana $L e c$.

961. ferruginea Lec. 


\section{Brachynus Web.}

- 362. americanus Lec. 963. janthinipennis $D e j$.

964. viridipennis Dej.

965. minutus Harr.

966. perplexus $D e j$.

967. medius Harr.

968. quadripennis Dej.

969. tenuicollis Lec.

970. conformis $D e j$.

971. eyanipennis Say.

972. lateralis $D e j$.

973. alternans $D e j$.

974. Deyrollii Laf.

975. tormentarius Lec.

976. ballistarius Lec.

977. fumans $F a b$.

977a. similis Lec.

978. cordicollis $D e j$.

979. rugipennis Chd.

980. puberulus Chd.

981. phreocerus Chd.

982. stygicornis Say.

983. kansanus Lec.

984. fidelis Lec.

985. Tschernikhii Mann.

986. carinulatus Mots.

987. costipennis Mots.

__ affinis Lec.

\section{Miscodera Esch.}

988. arctica Payk. americana Mann. Hardyi Chd.

989. insignis $\operatorname{Mann}$.

\section{Zacotus Lec.}

990. Matthewsii Lec.

\section{Chlænius Bon.}

991. ruficauda $C h d$.

992. herbaceus Chev.

993. viridifrons $E$ sch.

994. erythropus Germ.

995. fuscicornis Dej.

- 996. sericeus Forst.

$996 a$. regularis Lec.

997. Chaudoiri Horn.

998. cursor Chev.
999. orbus Horn.

1000. laticollis Say.

1001. diffinis Chd.

1002. platyderus Chd.

1003. restivus Say.

1004. viduus Horn.

1005. augustus Newm.

1006. cumatilis Lec.

1007. prasinus Dej.

1008. leucoscelis Chev.

1009. solitarius Say.

1010. obsoletus Lec.

1011. flaccidus Horn.

1012. vafer Lec.

1013. variabilipes Esch.

1014. nebraskensis Lec.

1015. simillimus $C h d$.

1016. glaucus Lec.

1017. nemoralis Say.

1017a. oxygonus Chd.

1018. tricolor $D e j$.

1019. brevilabris Lec.

1020. floridanus Horn.

1021. pennsylvanicus Say.

1022. texanus Horn.

- 1023. circumcinctus Say.

1024. maxillosus Horn.

1025. impunctifrons Say.

1026. insperatus Horn.

1027. harpalinus Esch.

1028. interruptus Horn.

1029. niger Rand.

1030. alternatus Horn.

1031. purpuricollis Rand.

1032. tomentosus Say.

1032a. amplus Lec.

1033. validus Chev.

\section{Anomoglossus Chd.}

- 1034. emarginatus Say.

1035. amønus Dej.

1036. pusillus Say.

\section{Brachylobus Chd.}

1037. lithophilus Say.

1038. caurinus Horn.

Lachnocrepis Lec.

1039. parallelus Say. 
Anatrichis Lec.

1040. minuta $D e j$.

Oodes Bon.

1041. amaroides $D c j$.

1042. americanus $D c j$.

1143. fluvialis Lec.

1044. Schaumi Chd.

10.45. cupreus Chd.

1046. Lecontei Chd.

1047. elegans $L e c$.

104\%. 14-striatus Chd.

10.49. texanus Ler.

\section{Evolenes Lec.}

1050. impressus $L \mathrm{er}$.

10j1. exaratus $D e$.

\section{Geopinus Lee.}

1052. incrassatus $I)$ ( )

\section{Pogonodaptus Horn.}

1053. piceus Horn.

\section{Nothopus Lec.}

10.54. zabroides $T e r$.

\section{Polpochila Sol.}

1055. capitata Chc.

\section{Cratacanthus Dej.}

105iti. dubius Bcunv. litoreus Cuscy. bisectus Cascy. texanus Cuscy.

Piosoma Lec.

1057. setosum Lee. 1058. alternatum Ife.

\section{Agonoderus Dej.}

1059. lineola Fub. maculatus $L f r$.

10150. infuscatus $D e j$.

1061. pallipes Fub. comma Fub. rugicollis $L e x$.
1062. partiarius Say.

1063. pauperculus $D e j$.

1064. indistinctus $D e j$.

106i5. testaceus Dej.

1066. micros Lec.

\section{Discoderus Lee.}

11367. parallelus Huld.

11668. impotens Lec.

11169. tenebrosus Lee.

1070. robustus Horn. pinguis Cusey.

11171. amœnus Lec.

\section{Stenomorphus Dej.}

Agrosomi Mén.

1072. californicus Mén.

1073. rufipes Lee.

\section{Gynandropus Dei.}

107t. hylacis Sey.

1175. elongatus Ler.

\section{Harpalus Lat.}

1076. dichrous $D \varepsilon j$.

107\%. vulpeculus Say.

1078. autumnalis Say.

107?. erraticus Suy.

111-i1. retractus Lee.

1081. amputatus Say.

11182. viridirneus Betur: renescens Casey. canonicus Cuscy. eonvictor Casey. lustralis Casey.

.... 11083. caliginosus Fub.

1084. faunus Stay.

1185. convivus $L e r$.

11)86. vagans $L e c$.

- 1087. pennsylvanicus $D E G$.

losion. compar Lece.

thrib. longior Kirby. longicollis Ler.

Iinit. erythropus $D e j$.

1118. megacephalus $L c c$.

[089. spadiceus $D C$.

1090. fulvilabris Menn.

1091. fallax Lcc. placidus Cusey. 
1092. pleuriticus Kirby.

1093. foveicollis Lec.

1094. herbivagus Say.

1095. somnulentus $D e j$.

1096. ventralis Lec.

1097. opacipennis Hald.

1098. nitidulus Chd.

1099. ellipsis Lec.

1100. vespertinus Casey.

1101. cautus $D e j$. defixus Walk.

1102. innocuus Lec.

1103. earbonatus Lec.

1104. rufimanus Lec.

1105. montanus Lec.

1106. Lewisii Lec.

1107. laticeps Lec.

1108. viduus $L e c$.

1109. fraternus Lec.

1110. funestus Lec.

1111. oblitus Lec.

1112. clandestinus Lec.

1113. furtivus $L e c$.

1114. stupidus Lec.

1115. ochropus Kirby.

1116. lustrans Casey. lucidus $\|$ Lec.

1117. basilaris Kirby.

1118. varicornis Lec.

1119. testaceus Lec.

1120. gravis Lec.

1121. alienus $L e c$.

1122. cordatus Lec.

1123. obliquus Horn.

_- albionicus Mann.

- curtatus Mann.

- liobasis Chd.

-.- oodioides Chd.

__ depressicollis Mots.

\section{Selenophorus Dej.}

1124. palliatus Fab. laesus Lec.

1125. pedicularius $D e j$. reneopiceus Casey. æreus Lec.

planipennis Lec. troglodytes $D e j$.

1126. fatuus Lec.

1127. breviusculus Horn.
1128. subtinctus Lec.

1129. iripennis $\$ a y$.

- 1130. gagatinus $D e j$.

perpolitus Casey.

viridescens Lec.

1131. opalinus Lec.

1132. fossulatus $D e j$.

1133. ovalis $D$ ej.

1134. ellipticus $D e j$. granarius $D e j$. pulicarius $D e j$.

\section{Stenolophus Dej.}

1135. carbonarius Brullé.

1136. spretus $D e j$.

1137. limbalis Lec.

¿ 1138. fuliginosus $D e j$.

1139. plebeius $D e j$.

1140. conjunetus Say. rotundatus Lec. scitulus Casey.

1141. anceps Lec.

1142. eincticollis Lec.

1143. unicolor $D e j$.

1144. flavipes Lec.

1145. ochropezus Say. gracilis Casey.

1146. dissimilis Dej.

1147. alternans Lec.

1148. tener Lec.

Acupalpus Lat.

1149. hydropicus Lec.

1150. carus Lec.

1151. flavilimbus Lec.

1152. longulus $D e j$.

1153. rectangulus Chd.

\section{Bradycellus Er.}

1154. linearis Lec.

1155. cognatus Gyll. axillaris Mann.

1156. neglectus Lec.

1157. cordicollis Lec.

-1158 . rupestris Say. congener Lec. parallelus Chd.

1159. tantillus Chd.

1160. nigriceps Lec. 
1161. ealifornicus Lec.

1162. rivalis Lec.

1163. lucidus Casey.

Tachycellus Moraw.

1164. nigrinus $D e j$. tibialis Kirby.

1165. Kirbyi Horn.

1166. atrimedius Say.

1167. nebulosus Lec.

1168. badipennis Hald.

1169. nitidus $D_{e j}$.

- symetricus Mots.

\section{Anisodactylus Dej. Amphasia Newm. Anisotarsus Chd. Gynandrotarsus Laf. Spongopus Lec. Xestonotus Lec.}

1170. strenuus Forn. 1171. dilatatus $D e j$. 1172. obtusus Lec. 1173. pilosus Horn. 1174. immanis Horn. 1175. brunneus $D e j$. 1176. piceus Mén. 1177. harpaloides Laf. 1178. dulcicollis Laf. 1179. opaeulus Lec. 1180. rusticus Dej. Śá! 1181. carbonarius Say. 1182. furvus Lec. 1183. semipunctatus Lec: 1184. consolurinus Lec.
1185. californicus $D e j$.

1186. interpunctatus Kirby.

1187. agricola Say.

L- 1188. Harrisii LCC.

1189. nigerrimus $D_{\ell j}$. opacus Cascy.

1190. nigrita $D e j$.

1191. melanopus Hald.

1192. arizonae Casey.

1193. nivalis Horn.

1194. discoideus $D e j$.

1195. baltimorensis Say.

1196. pitychrous Lce.

1197. porosus Mots. alternans Mots.

1198. verticalis Iec.

1199. maculicornis Chd.

1200. flebilis Lec.

1201. piceus Mén. L'C

1202. terminatus Say.

[203. agilis $D e j$.

1204. nitidipennis $L \Subset c$.

1205. lætus $D_{e j}$.

1206. conus Say.

1207. amaroides $L e c$.

1208. lugubris $D(j$. manhattanis Cascy.

1209. sericeus Harr.

1210. interstitialis Say.

_- pallidus Mots.

Pseudomorpha Kirby.

1211. excrueians Kirby.

1212. Cronkhitei Horn.

1213. Behrensii Horn.

1214. angustata Horn.

\section{IMIIIZUIDA.}

Amphizoa Lec.

1215. insolens LCc. 1215a. Josephi Matth.

1216. Lecontei Matth.

\section{IIIPIIID E.}

Brychius Thom.

1217. Hornii Cr.

Haliplus Lat.

$\theta$ 1218. fasciatus $A u b e ́$.

1219. tumidus Lec.
1220. punctatus Aubé.

1221. triopsis Say.

1222. borealis Lec.

1223. concolor Lec.

1224. Lewisii $\mathrm{Cr}$.

1225. cribrarius tec. 
1226. ruficollis $D e G$.

1227. longulus Lec.

Cnemidotus Er.

1228. callosus Lec.
1229. simplex Lec.

1230. festivus Wehnke.

1231. 12-punctatus Say.

1232. muticus Lec.

1233. edentulus Lec.

\section{$\checkmark$ DYTISCID E.}

\section{Colpius trec.}

1234. inflatus Lec.

\section{Canthydrus Sharp.}

Suphis Aubé.

1235. puneticollis $C r$.

1236. semipunctatus Lec.

1237. bicolor Say.

1238. gibbulus $A u b e ́$.

1239. lineatus Horn.

\section{Hydrocanthus Say.}

\& 1240. iricolor Say.

Notomicrus Sharp.

1241. nanulus Lec.

Laccophilus Leach.

〉1242. maculosus Gcrm.

1243. decipiens Lec. fusculus Sharp.

1244. proximus Say.

1245. Iateralis Horn.

1246. pictus Lap.

1247. insignis Sharp.

1248. fasciatus Aubé.

1249. americanus $A u b e ́$.

1250. terminalis Sharp.

1251. mexicanus Aubé. atristernalis $\mathrm{Cr}$.

1252. 4-lineatus Horn.

1253. undatus $A u b e ́$.

1254. gentilis $L e c$.

1255. pumilio Lec.

Hydrovatus Mots.

1256. cuspidatus Germ.

1257. pustulatus Melsh.

1258. brevipes Sharp.

1259. compressus Sharp.

1260. Hornii $\mathrm{Cr}$.
Desmopachria Bab.

1261. granum Lec.

1262. convexa Aubé.

1263. latissima Lec.

1264. dispersa $\mathrm{Cr}$.

\section{Bidessus Sharp.}

1265. exiguus $A u b e ́$.

1266. cinctellus Lec.

1267. pictodes Sharp.

1268. seminulum Lec.

1269. plicipennis $\mathrm{Cr}$.

1270. flavicollis Lec.

1271. obesus Sharp.

1272. discretus Sharp.

1273. pulicarius Aubé. inconspicuus Lec.

1274. texanus $\mathrm{Cr}$.

1275. affinis Say. macularis Lec. nanus $A$ ubé. nigrinus Casey.

127t. subtilis Lec.

127 . amandus $L e c$.

1278. lacustris Say.

1279. fuscatus $\mathrm{Cr}$.

1280. pullus Lec.

1281. granarius Aubé.

\section{Celina Aubé.}

1282. angustata Aubé.

1283. grossula Lec.

\section{Coelambus Thom.}

1284. inæqualis $F a b$.

1285. punctatus Say.

1286. hydropicus Lec.

1287. farctus Lec.

1288. acaroides Lec.

1289. turbidus Lec.

1290. lutescens Lec. fumatus Sharp. 
1291. patruelis Lec.

1292. suturalis Lec.

1293. sellatus Lec.

1294. medialis Lec.

1295. ovoideus Lec.

1296. masculinus $\mathrm{Cr}$.

1297. nubilus Lec.

1298. unguicularis $\mathrm{Cr}$.

1299. infuscatus Sharp.

1300. fraternus $L e c$.

1301. dissimilis Harr. $G: / /$

1302. impressopunctatus Sch. picatus Kirby.

\section{Deronectes Sharp.}

1303. depressus Fab. rotundatus Lec.

1304. catascopium Say. prosternalis Sharp. suffusus Sharp.

1305. griseostriatus $D \ell G$.

1306. striatellus Lec. pulcher || Mots.

\section{Hydroporus Clairv.}

1307. aulicus $A u b e ́$.

1308. diversicornis Sharp.

130y. concinnus Lec.

1310. pulcher Lec. integer Sharp.

1311. vittatipennis G. \& $H$. lineolatus $\|$ Lec.

1312. cimicoides Sharp.

1313. mellitus Lec.

1314. undulatus Say. oppositus Say. labratus Sharp.

1315. scrutator Sharp.

1316. Iynceus Sharp.

1317. spurius Lec. peltatus Sharp.

1318. clypealis Sharp.

1319. anticus Sharp.

1320. eonsimilis Lec.

1321. eruditus Lec.

1322. republicanus Sharp.

1323. proximus Aubé.

1324. vitiosus Lec.
1325. vittatus Lec.

1326. sericeus Lec.

1327. dimidiatus $G$. $\& H$. semirufus $\|$ Lec.

1328. striatopunetatus Melsh.

1329. hybridus $A u b e ́$.

1330. mixtus Lec.

1331. solitarius Sharp.

13.32. eximius Mots.

1333. addendus $\mathrm{Cr}$.

1334. funereus $\mathrm{Cr}$.

1335. mexicanus Sharp.

1336. alpinus Payk.

1337. 12-lineatus Lec.

1338. septentrionalis Gyll. scitulus Lec.

1339. picturatus Horn.

1340. rivalis Gyll.

obesus Lec.

congruus Lec.

1341. quadrimaculatus Horn.

1342. occidentalis Sharp.

1343. caliginosus Lec. puberulus Lec.

1344. obscurus Sturm.

1345. tenebrosus Lec. despectus Sharp. perplexus Sharp. rusticus Sharp. subpubescens Lec.

1:46. signatus Mann.

1:iti. longiusculus $G$. \& $H$.

1::48. glabriusculus Aubé.

1:i49. tartaricus Lec.

1:350. morio Sharp.

13.51. notabilis Lec.

1:i.j2. tristis Payk. subtonsus Lec.

13i:. entractulus Mann.

1354. rufinasus Mann.

1:3j. vitulus $\mathrm{Er}$.

1356. humeralis $A u b e ́$.

1:557. fortis Lec.

1:3\%. americanus $A u b e ́$.

1:559. dichrous Melsh.

1:i6i). inornatus Sharp.

1:361. niger Say.

13ti2. latifrons Sharp.

1:36. rufilabris Sharp.

1364. ruficeps $A$ ubé. 
1:30.). axillaris tee.

1366. modestus $A$ ubé.

1367. stagnalis G. \& $H$. collaris Lec. persimilis $\mathrm{Cr}$.

1368. oblitus Aubé.

1369. terminalis Sharp.

1370. vilis Lec.

1371. palliatus Horn.

1372. latehrosus Lec.

1373. Hardyi Sharp.

137t. Belfragei Sharp.

1375. difformis Lec.

1376. oblongus Steph. conoideus Lec.

1377. laccophilinus Lec.

- planatus Manu. truncatus $\operatorname{Mann}$. impressifrons Mots.

\section{Ilybius Er.}

1378. ater $D e G$.

1379. subæneus $E r$.

1380. pleuriticus Lec.

1381. suffusus $\mathrm{Cr}$.

1382. viridiæneus $C r$.

1383. inversus Sharp.

1381. 4-maculatus Iee. Aub É

1385. ignarus Lec.

1386. angustior Gyll. picipes Kirby.

1387. fuliginosus $F a b$.

1388. discedens Sharp.

1389. biguttalus Germ.

1390. fraterculus Lec.

1391. laramæus Lec.

1392. confusus Aubé.

1393. oblitus Sharp.

1394. assimilis Kirby.

\section{Coptotomus Say.}

1395. obscurus Sharp.

1396. interrogatus $F a b$.

1397. longulus Lec:

\section{Hydrotrupes Sharp.}

1398. palpalis Sharp.
Ilybiosoma $\mathrm{Cr}$.

-1399. bifarius Kirby.

1400. regularis Lec.

Copelatus Er.

1401. Chevrolatii Aubé.

1402. glyphicus Say.

Agabinus Cr.

1403. glabrellus Mots.

Matus Aubé.

1404. bicarinatus Say.

Agabetes $\mathrm{Cr}$.

1405. acuductus Harr.

Agabus Leach.

Anisomera Brullé.

Gaurodytes Thom.

140h. cordatus Lec.

1407. rectus Lec.

1408. intersectus $\mathrm{Cr}$.

1409. hypomelas Mann.

1410. parallelus Lec.

1411. seriatus Say.

1412. suturalis $\mathrm{Cr}$. perplexus Sharp.

1413. lugens Lec.

1414. obtusatus Say.

1415. planatus Sharp.

1416. brevicollis Lec.

1417. stagninus Say.

1418. confertus Lec. Walsinghami $\mathrm{Cr}$.

1419. semivittatus Lec.

1420. texanus Sharp.

1421. punctatus Melsh.

1422. æruginosus $A u b e ́$.

1423. semipunctatus Kirby.

1424. inseriptus $\mathrm{Cr}$.

1425. punctulatus $A u b e ́$. æneolus $\mathrm{Cr}$.

1426. stridulator Sharp.

1427. tæniolatus Harr.

s'1428. disintegratus $\mathrm{Cr}$.

1429. lineellus Lec. 
14:iu. congener Payk.

14:i1. ambiguus Say. ovoideus Lec. $_{\text {tec }}$

14::2. muticus Sharp.

14iii. anthracinus Mann. seapularis Mann.

T434. subfuscatus Sharp.

1435. Austinii Sharp.

1436. strigulosus $C r$. nantrs Lec.

1437. morosus Lec.

1438. infuscatis $A u b e ́$.

1439. reticulatus Kirby.

1440. obsoletus Lcc.

1441. Lecontei $\mathrm{Cr}$.

1442. griseipennis $L e c$.

1443. obliteratus Lec.

1444. erythropterus Say.

1445. arcticus $P a y k$.

1446. nigroæneus $\mathrm{Er}$. lutosus $\mathrm{Cr}$.

1447. gagates $A u b e ́$.

1448. discors Lcc.

1449. tristis Aubé.

1450. clavatus $L c e$.

1451. subopacus Mann.

1452. terminalis Melsh.

1453. leptapsis $L \in c$.

1454. longulus Lce.

\section{Scutopterus Esch.}

1455. angustus Lec.

1456. Hornii $C r$.

Rhantus Esch.

1457. divisus $A u b e ́$.

1458. flavegriseus $\mathrm{Cr}$. longipes sharp. obscurus Sharp.

1159. binotatus Harr. plebejus Sharp.

Ifill. anisonyehus $\mathrm{Cr}$.

Itil. atricolor Aubé.

I Hiz. calidus Fab.

$146 \%$. consimilis Mots.

11it. sinuatus Lec.

14ti5. notatus $F$ itb. sericans Sharp.

1fili. bistriatus Bergst.

1 HiT. tostus LCc. discedens Shrrp.
Colymbetes Clairv.

Cymatopterus Esch.

1468. Paykulli Er.

1469. longulus Lec.

1470. obscuratus Mann.

1471. seminiger Lec. inæqualis Horn.

1472. strigatus Lec.

1473. grøalandicus $A u b e ́$.

1474. seulptilis Harr. exaratus Lec. rugipennis Sharp

1475. Thomsoni Sharp.

Eretes Lap.

1476. sticticus Linn.

Hydaticus Leach.

1477. stagnalis Fab. americanus Sharp. modestus Sharp.

1478. Irvipennis Thom.

1479. piceus $L e c$.

1480. bimarginatus Say.

Dytiscus Linn.

4481. fasciventris Say.

1482. hybridus Aubé.

1483. verticalis Say.

7 1484. marginicollis Lcc.

1485. sublimbatus Lec.

Cordieri Aubé.

1486. marginalis Linn.

1487. circumcinctus $A h r$. ? anxius Mann.

1488. parvulus Mann.

148!. vexatus Sharp.

1490. dauricus Gebl. confluens Lec.

1491. Harrisii Kirby.

Acilius Leach.

1492. semisulcatus Aubé:

1493. fraternus $H a r r$.

1494. mediatus Say.

Thermonectes Esch.

7 1495. ornaticollis Aubé. 1495 a. nigrofasciatus $A u b c$. 
1496. basilaris Harr. 1496a. intermedius $\mathrm{Cr}$. 14966. latecinctus Lec. 1497. marmoratus Hope.

Graphoderes Esch.

1498. liberus Say.

1499. fasciatocollis Harr. perplexus Sharp. elatus Shavp.

1500. occidentalis Horn.

\section{Cybister Curt.}

1501. Olivieri $\mathrm{Cr}$.

1502. fimbriolatus Say.

1503. ellipticus Lec.

1504. explanatus Lcc.

\section{GYIRINID F.}

Gyrinus Linn.

1505. minutus $F a b$.

1506. rockinghamensis Lec.

1507. confinis Lec.

1508. fraternus Coup.

1509. æneolus Lec.

1510. limbatus Say.

1) 1511. dichrous Lec.

1512. elevatus Lec.

1513. ventralis Kirby.

1514. aquiris Lec.

1515. plicifer Lec.

1516. consobrinus Lec.

1517. maculiventris $L e c$.

1518. canadensis Reg.

1519. affinis $A u b e ́$.

1520. pernitidus Lec.

1521. analis Say.

1522. Sayi Aubé.

1523. marinus Gyll. opacus Sahlb.

1524. pectoralis Lec.

1525. impressicollis Kirby.
1526. gibber Lec.

1527. borealis Aubé.

1528. lugens Lec.

1529. picipes $A$ ubé.

1530. corpulentus Reg.

1531. pareus Say.

- lateralis Aubé.

-_ fuscipes Mots.

Dineutes MacL.

1532. vittatus Germ.

1533. sublineatus Chcv.

1534. discolor Aubé. angustus $L e c$.

1535. serrulatus $L e c$.

1536. assimilis $A u b \dot{e}$.

1537. analis Reg.

1538. emarginatus Say.

1539. carolinus Lec.

Gyretes Brullé.

1540. sinuatus $L e c$. compressus Lec.

\section{IYY D ROPH HLIDA.}

Helophorus Fab.

1541. fortis Lec.

1542. oblongus Lec.

1543. lacustris Lec.

1544. obscurus Lec.

1545. nitidulus Lec.

1546. linearis $L e c$. ? pusillus Mots.

1547. angustulus Mann.
1548. granularis Mots.

1549. obsoletesulcatus Mots.

1550. lineatus Say. pallens Casey. ? ventralis Mots.

1551. inquinatus Mann.

1552. auricollis Esch.

1553. tuberculatus Gyll. 
Hydrochus Leach.

1554. scabratus Muls.

1555. callosits Lec.

1556. foveatus Hald.

1557. rugosus Muls.

1558. inæequalis Lec.

1559. excavatus Lec.

1560. subcupreus Rand.

1561. varjolatus Lec.

1562. squamifer Lec.

1563. impressus Zimm.

1564. vagus Lec.

1565. simplex Lec.

\section{Epimetopus Lac.}

1566. costatus Lec.

\section{Ochthebius Leach.}

1j67. puncticollis Lec.

156:. discretus Lec.

1569. rectus Lec.

1570. eribricollis Lec.

1571. attritus Lec.

1572. simplex Lec.

157. tuberculatus $L e c$.

1574. nitidus Lec.

1575. lævipennis Lec.

1576. foveicollis $L e c$.

1577. benefossus Lec.

1578. sculptus Lec.

1579. Holmbergi Makl.

15.80. lineatus Lec.

1581. interruptus Lec.

\section{Hydraena Kug.}

1582. pennsylvanica Kies.

1583. punctata Lec.

1584. marginicollis Kies.

Hydrophilus Geoft:

Tropisternus Sol.

$>1585$. ovatus G.\&H.

1586. triangularis Say.

1587. insularis Lap.

1588. limbalis Lec.

1589. nimbatus Say. -

- 1590. mixtus Lec.

1591. glaber Hbst.

1592. californicus Lec.
1593. sublevis Lec.

1594. ellipticus Lcc.

1595. striolatus Lec.

\section{Hydrocharis Lat.}

1596. glancus Lec.

Y.1597. obtusatus Say.

1598. castus Say.

Berosus Leach.

1599. maculosus Mann.

1600. punctatissimus $L \epsilon C$ 。

1601. Salvini Sharp.

1602. miles Lec.

1603. pugnax Lec.

1604. emarginatus Horn.

1605. styliferus Horn.

1606. aculeatus Lec.

1607. subsignatus Lec.

1608. pantherinus Lec.

1609. peregrinus Hbst.

1610. immaculatus Zimm.

1611. exilis Lec.

1612. exiguus Say.

1613. infuscatus Lec.

1614. striatus Say.

1615. rugulosus Horn.

Derallus Sharp.

1616. altus Lec.

Chæetarthria Steph.

1617. atra Lee.

1618. nigrella Lec.

1619. pallida Lec.

Limnebius Leach.

Limnocharis Horn.

1620. piceus Horn.

Laccobius Er.

1621. agilis Rand.

1622. ellipticus Lec.

Philhydrus Sol.

1623. normatus Lec.

1624. carinatus Lec.

1625. fucatus Horn. 
1626. Lebulosus Say.

1627. pectoralis Lec.

1628. eristatus Lec.

-1629. bifidus Lec.

1630. ochraceus Mels.

16.3. reflexipennis Zimm. angustulus Cusey.

1632. consors Lec.

1633. cinctus Say.

16i24. diflusus Lec.

1635. fuscus Mots.

1636. perplexus Lec.

1637. latiuseulus Mots.

1638. obtusiuseulus Mots.

\section{Hydrocombus Sharp.}

1639. maculicollis Muls.

1640. punctatostriatus Hor".

164t1. imbellis Lee.

? dorsalis Mots.

1642. fimbriatus Melsh.

1643. lacustris Lec.

1614. rotundatus Say.

\section{Hyđrobius Leach.}

1645. tessellatus Ziegl.

1646. seabrosus Horn.

1647. latus Horn.

1648. turfindus Lec.

1649. cuspidatus Lec.

1650. castaneus Lec.

1651. feminalis Lec.

1652. globosus Say. melænus Germ.

1653. fuscipes $\operatorname{Linn}$.

1654. rufiventris Horn.

1655. infuscatus Mots.

1656. digestus Lec.

1657. subcupreus Say.

1658. despectus Lec.

1659. dissimilis Horn.

1660. suturalis Lec.

Helopeltis Horn.

1661. larvalis Horn.

\section{Sphaeridium Fab.}

1662. scarabæoides Linn.

\section{Dactylosternum Woll.}

1663. abdominale Fab.

\section{Cercyon Leach.}

1664. pubescens Lec.

1665. fimbriatum Mann.

1666. Hlavipes $F a b$.

1667. nigricolle Say.

1668. lunigerum Mann.

1669. limbatum Mann.

1670. fulvipenne Mann.

1671. naviculare $Z ı \mathrm{~mm}$.

1672. melanocephalum Linn.

1673. apicale Say.

1674. centromaculatum Sturm.

1675. prætextatum Say.

1676. adumbratum Mann.

1677. ocellatum Say.

1678. pygmæum Ill.

1679. unipunctatum Linn.

1680. anale Payk.

1681. posticatum Mann.

1682. nanum Melsh.

1683. miniseulum Melsh.

1684. capillatum Lec.

\section{Phœnonotum Sharp.}

1685. extriatum Say.

1686. semiglobosum Zimm.

\section{Cyclonotum Er.}

1687. cacti Lec.

1688. palmarum $\mathbf{S z}$.

Megasternum Muls.

1689. costatum Lec.

Cryptopleurum Muls.

1690. vagans Lec.

\section{PLATYISYILEID.E.}

Platypsylla Ritsema.

1691. castoris Ritsema. 


\section{IAEPTIN ID.E.}

Leptinus Müll.

1692. testacens Müll.
Leptinillus Horn.

1693. validus Horn.

\section{SILPIIDA.}

Necrophorus Fab.

1694. carolinus Linn.

1695. amerieanus Oliv.

1696. Sayi Lap.

Q 1697. orbicollis Say.

\& 1698. marginatus Fab.

1699. obscurus Kirby.

1700. guttula Mots.

hecate Bland.

$\theta$ 1701. pustulatus Her.sch.

1701a. Melsheimeri Kirby.

1701b. nigritus Mann.

1702. vespilloides Hbst. defodiens Kirby.

hebes Kirby.

1702a. pollinetor Lec.

1703. tomentosus Web.

\section{Silpha Linn.}

7 1704. surinamensis Fab.

1705. truncata Say.

> 1706. lapponica Hbst.

1707. trituberculata Kirby.

1708. inæqualis $F a b$.

1709. noveboracensis Forst.

1710. americana Linn.

7 1711. ramosa $S a y$.

1712. opaca Linn.

1713. bituberosa Lec.

\section{Necrophilus Lat.}

1714. Pettitii Horn. subterraneus $\ddagger$ Horn

y 1715. hydrophiloides Mann.

Pelates Horn.

1716. latus Mann.

\section{Pteroloma Gyll.}

1717. Forstroemii Gyll.

1718. tenuicornis Lec.
Agyrtes Fröh.

1719. longulus Lec.

Sphaerites Duft.

1720. glabratus $F a b$. politus Mann.

Lyrosoma Manu.

1721. opaca Mann.

Pinodytes Horn.

1722. eryptophagoides Mann.

Platycholeus Horn.

172\%. leptinoides $\mathrm{Cr}$.

\section{Catoptrichus Murr.}

1724. Frankenhaeuseri Mann.

\section{Choleva Lat.}

1725. egena Horn.

1726. luridipennis Mann.

1727. atsiosa Horn.

1728. simplex Say.

1729. basillaris Say. brunnipennis Mann. Spencianus Kirby.

1731. elavicornis Lec.

1731. decipiens Horn.

1732. terminans Lec.

Echinocoleus Horn.

1733. setiger Horn.

\section{Prionochaeta Horn.}

1734. opaca Say.

Ptomaphagus 111.

1735. consobrinus Lec.

1736. fisus Horn. 
1737. californicus Lec.

1738. nevadicus Horn.

1739. Ulkei Horn.

1740. oblitus Eec.

1741. pusio Lec.

1742. parasitus Lec.

1743. brachyderus Lec.

Adelops Tellk.

1744. hirtus Tellk.

\section{- Colon Hbst.}

1745. bidentatum Sahlb.

1746. paradoxum Horn.

1747. Hubbardi Horn.

1748. dentatum Lec.

1749. celatum Horn.

1750. putum Horn.

1751. magnicolle Mäkl.

1752. pusillum Horn.

1753. clavatum Miikl.

1754. inerme Mäkl.

1755. thoracicum Horn. decoris Casey.

1756. asperatum Horn.

1757. nevadense Horn.

Triarthron Maerk.

1758. Lecontei Horn. cedonulli Schauf.

1759. pennsylvanicum Horn.

Hydnobius Schm.

1760. Matthewsii $\mathrm{Cr}$. ? pallidus Say.

1761. strigilatus Horn.

1762. arizonensis Horn.

1763. longulus Lec.

1764. substriatus Lec.

1765. latidens Lec.

1766. obtusus Lec.

\section{Anogdus Lee.}

1767. capitatus Lec.

Anisotoma Ill.

1768. alternata Melsh.

1769. humeralis Horn.

1770. valida Horn.
1771. assimilis Lec.

1772. punctatostriata $K$ irby. lreta $M(z n n$.

1773. difficilis Horn.

1774. collaris Lec.

1775. curvata Mann. morula Lec.

1776. conferta Lec.

1777. paludicola $\mathrm{Cr}$.

1778. strigata Lec.

1779. obsoleta Melsh.

1780. ecarinata Horn.

- lateritia Mann.

Colenis Er.

1781. impunctata Lec.

Liodes Lat.

1782. globosa Lec.

178.3. polita Lec.

1784. discolor Melsh.

1785. Blanchardi Horn.

1786. obsoleta Horn.

1787. basalis Lec.

1787a. dichroa Lec.

1788. geminata Horn.

1789. confusa Horn.

\section{Cyrtusa Er.}

1790. picipennis Lec.

1791. blandissima Zimm.

1792. egena Lec.

impubis Zimm.

\section{Isoplastus Horn.}

1793. fossor Horn.

\section{Agathidium 111.}

1794. oniscoides Beauv.

1795. exiguum Melsh.

1796. dentigerum Horn.

1797. californicum Horn.

1798. revolvens Lec.

1799. sexstriatum Horn.

1800. bistriatum Horn.

1801. estriatum Horn.

1802. repentinum Horn.

1803. concinnum Mann. effluens Mann. 
1804. rotundulum Mann.

1805. angulare Mann.

1806. politum Lec.

1807. pulchrum Lec. mandibulatum Mann.

1808. difforme Lec.

Aglyptus Lec.

1809. levis Lec.

Empelus Lec.

1810. brunneipennis Mann.
Calyptomerus Redt.

1811. oblongulus Mann.

Clambus Fisch.

1812. gibbulus Lec.

1813. puberulus Lec.

1814. vulneratus Lec.

1815. seminulum Horn.

SCY II ENIDE.

Chevrolatia Duval.

1816. amona Lec:

\section{Scydmæenus Lat.}

1817. subpunctatus Lec.

1818. marix Lec.

1819. cribrarius Lec.

1820. perforatus Schaum.

1821. sparsus Lec.

1822. angustus Lec.

1823. cautus Lec.

1824. magister Lec.

1825. mississippicus Zimm.

1826. Havitarsis Lec.

1827. fossiger Lec.

1828. divisus $\mathbf{S z}$.

1829. capillosulus Lec.

1830. basalis Lec.

1831. hirtèllus Lec.

1832. analis Lec.

1833. brevicornis Suy.

1834. rasus Lec.

1835. obscirrellus Lec.

1836. elavatus Lec.

1837. pyramidalis Liec.

1838. clavipes Say.

1839. consobrinus Lec.

1840. bieotor tec:

1841. Salinator Ler.

1842. fatuus Lec.
1843. misellus Lec.

1844. gravidus Lec.

1845. fulvus Lec.

1846. graeilis Lec.

1847. biformis Makl.

1848. deformatus Horn.

1849. californicus Mots.

Eumicrus Lap.

1850. Motschulskii Lec.

1851. grossus Lec.

1852. vestalis Cissey.

1853. punctatus Cusey. $=185 \mathrm{~L}$

Cholerus Thom.

1854. Zimmermanni Schaum.

\section{Cephennium Mùll.}

1855. corporosum Lec.

Eutheia Steph.

1856. colon Horn.

1857. scitula Makl.

1858. impressa Lec.

1859. longula Lec.

Brathinus Lec.

1860. nitidus Lec.

1861. varicornis Lec.

\section{IPSIIA AMIDE.}

Adranes luec.

1862. coecus Lec.

1863. Lecontei Brend. 
Fustiger Lec.

1864. Fuchsii Brend.

\section{Atinus Horn.}

1865. monilicornis Brend.

\section{Ceophyllus Lec.}

1866. monilis Lec.

\section{Cedius Lec.}

1867. Ziegleri Lec.

1868. spinosus Lec.

\section{Tmesiphorus Lec.}

1869. costalis Lec.

1870. carinatus Say.

Ctenistes Reichenb.

1871. piceus Lec.

1872. pulvereus Lec.

1873. Zimmermanni Lec.

1874. consobrinus Lec.

\section{Tyrus Aubé.}

1875. humeralis Aubé.

\section{Cercocerus Lec.}

1876. batrisioides Lec.

\section{Pselaphus Hbst.}

1877. Erichsonii Lec.

$1877 a$. longiclavus Lec.

Tychus Leach.

1878. longipalpus Lec.
testaceus Casey.
1879. minor Lec.
1880. puberulus Lec.
1881. cognatus Lec.
1882. tenellus Lec.
1883. bythinioides Brend.

\section{Bythinus Aubé.}

1884. carinatus Brend. 1885. zonatus Brend.

Decarthron Brend.

1886. abnorme Lec.
1887. exsectum Brend.

1888. stigmosum Brend.

1889. Iongulum Brend.

1890. formiceti $L e c$.

1891. strenuum Brend.

1892. cornutum Brend.

\section{Batrisus Aubé.}

Arthmius Lec.

1893. globicollis Lec.

gracilior Casey

1894. Ionse Lec.

1895. juvencus Brend.

1896. confinis Lec.

1897. armiger Lec.

1898. monstrosus Lec.

1898a. ferox Lec.

1898b. cristatus Lec.

1899. frontalis Lec.

1900). scabriceps Lec.

1901. Schaumii Aubé.

1902. riparius Say.

1903. globosus Lec.

1904. nigricans Lec.

1905. albionieus $A u b e ́$.

1906. spretus Lec.

1907. aterrimus Casey.

1908. bistriatus Lec.

1909. denticollis Casey.

1910. virginiæ Casey.

1911. lineaticollis $A u b e ́$.

1912. simplex Lec.

Pselaptus Lee.

191:. Belfragei Lec.

Bryaxis Leach.

1914. sanguinea Leach.

1915. conjuncta Lec.

1916. Brendelii Horn.

1917. foveata $L e c$.

1918. perforata Brend.

1919. Belfragei Lec.

1920. dentata Say.

1921. Ulkei Brend.

1922. illinoiensis Brend.

1923. abdominalis $A u b e ́$.

1924. intermedia Brend.

1925. Horidana Brend. 
1926. luniger Lec.

1927. eavicornis Brend.

1928. gemmifer Lec.

1929. radians Lec.

1930. divergens Lec.

1931. atlantica Brend.

1932. rubicunda $A u b e ́$.

1933. fucilis Casey.

1934. congener Brend.

1935. graciliв Casey.

1936. trigona Lec.

1937. scabra Brend.

1938. puncticollis Lec.

1939. sagax Lec.

1940. propinqua Lec.

1941. albionica Mots.

1942. complectens Lec.

1943. subtilis Lec.

1944. deformata Lec.

1945 compar Lec.

1946. tumida Lec.

1947. tomentosa Lec. inopia Casey.

1948. inornata Brend.

Scalenarthrus Lec.

1949. Hornii Lec.

\section{Eutrichites Ijec.}

1950. Zimmermanni Lec.

Eupsenius Lec.

1951. glaber Lec.

1952. rufus Lec.

Faronus Aubé.

1953. tolulæ Lec.

1954. isabellæ Lec.

1955. parviceps Mäkl.

Trichonyx Chd.

1956. striatus Lec.

\section{Rhexius Lec.}

1957. insenlptus Lec.

1958. substriatus Lec.

\section{Rhinoscepsis Lec.}

1959. bistriatus Lec.

\section{Trimium Aubé.}

1960. foveicolle Lec.

1961. globifer Lec.

1962. impunctatum Brend.

1963. ealifornicum Lec.

1964. puncticolle Lec.

1965. discolor Lec.

1966. parvulum Lec.

1967. convexulum Lec.

1968. simplex Lec.

1969. dubium Lec.

1970. americanum Lec.

_- clavicorne Makl.

Euplectus Leach.

1971. canaliculatus Lec.

1972. pumilus Lec.

1973. debilis $L e c$. tenuis Lec.

1974. longicollis Casey.

1975. ditticilis Lec.

1976. congener Casey.

1977. sexualis Casey.

1978. spinifer Casey.

1979. linearis Lec.

1980. interruptus Lec.

1981. confluens Lec.

1982. pertenuis Casey.

1983. eavicollis Lec.

1984. crinitus Brend.

1985. capitulum Casey.

1986. decorus Casey.

1987. areuatus Lec.

1988. leviceps Casey.

1989. ruficeps Lec. integer Lec.

Thesium Casey.

1990. cavifrons Lec.

1991. laticolle Casey.

Faliscus Casey.

1992. bicanalis Casey.

Nicotheus Casey.

1993. tibialis Casey.

Eutyphlus Lec.

1994. similis Lec. 
STA PUYLINID $A$.

Falagria Mann.

1995. sentellaris Lec.

1996. cingulata Lec.

1997. læviuscula Lec.

1998. quadriceps Lec.

1999. partita $L e c$.

2000. vaga Lec.

2001. cavipennis Lec.

2002. bilobata Say.

2003. dissecta $E r$.

2004. venustula $E r$.

Echidnoglossa Woll.

\section{Hoplandria Kraatz.}

2005. pulchra Kraatz. 2006. lateralis Melsh.

\section{Homalota Mann.}

2007. opaca Lec.

2008. plana Gyll.

2009. trimaculata Er.

2010. remula Er.

2011. dichroa Grav.

2012. vestigialis $E r$.

2013. festinans $E r$.

2014. luteola $E r$.

2015. Haveola Melsh.

2016. silacen $E r$.

2017. recondita $E r$.

2018. ambigua $E r$.

2919. polita Melsh.

2020. coriaria Kraatz.

2021. modesta Melsh.

2022. analis Grav.

2023. lividipennis Mann.

2024. pedicularis Melsh.

2025. granularis Mann.

2026. Maritima Mann.

2027. picipennis Mann.

2028. Irevieollis ITakl.

2029. cursor $\boldsymbol{M} \ddot{a} k l$.

2030. nitens Mäkl.

2031. moesta Mükl.

2032. pratensis Mäkl.

2033. geniculata $M a k k l$.

2034. planaris Mikl.
2035. breviuscula Mäkl.

2036. comparabilis Mïkl.

2037. littoralis $\boldsymbol{M} \ddot{k} k$ l.

2038. vasta $M i k l$.

2039. fueicola Miihl.

2040. sordida Marsh.

- ? indenta Say.

— ? propera Sry.

_- ? falsifica Say.

___ ? simplicicollis Say.

—— ? minima Say.

-_ ? quadripunctata Say.

_- ? pallitarsis Kirby.

- lateralis Mäkkl.

Lomechusa Grav. Atemeles Steph.

2041. cava Lec.

Callicerus Grav.

Tachyusa Er.

2042. pygmæa Sachse.

2043. cavicollis Lec.

2044. nigrella Lec.

2045. baltifera Lec.

2046. gracillima Lec.

Myrmedonia Er.

2047. angularis Mäkl.

2048. rudis $L e c$.

2049. Fauvelii Sharp.

Pelioptera Kraatz.

2050. ? gigantula Lec.

Phloeopora Er.

2051. lakens $\mathrm{Er}$.

Ilyobates Kraatz.

Calodera Mann.

Ocalea Er.

Polystoma Steph. Emplenota Casey

2052. maritima Casey. 
Aleochara Grav.

2053. lata Grov.

2054. brachypterus Fourc.

2055. bimaculata Grav.

2056. (astameipennis Mann.

2057. suleicollis Mann.

2058. cognata Mäkl.

2059. nitida Grav. anthomyir Sprague.

Maseochara Sharo.

2060. valida Lec.

Homoeusa Kraatz.

2061. expansa Lec.

Haploglossa Kraatz.

Dasyglossa Kraatz.

Thiasophila Kruatz.

Oxypoda Mann.

2062. sagulata $E r$.

2063. irrasa Mäkl.

2064. minuta Sachse.

Euthorax Sol.

Myrmecochara Kraatz.

2065. pictipennis Krratz.

Polylobus Sol.

Autalia Mann.

Eudera Fauv.

2066. geniculata Mïkl.

Phytosus Curt.

2067. littoralis Horn.

\section{Stenusa Kratz.}

2068. alternans Sachse. 2069. gracilis Sachse.
Silusa Er.

Placusa Er.

2070. despecta $E r$.

Bolitochara Mann.

2071. notata $M \ddot{k} k$.

Euryusa Er.

2072. obtusa Lec.

Philotermes Kraatz.

2073. pilosus Kraatz.

2074. pennsylvanicus Kraatz.

2075. Fuchsii Kraatz.

Leptusa Kraatz.

Oligota Maun.

2076. pedalis Lec.

-... ? exigua Say.

Gyrophaena Mann.

2077. vinula $E r$.

2078. dissimilis Er.

2079. flavicornis Melsh.

2080. corruscula Er.

2081. socia Er.

2082. aflinis Fauv.

- ? rufit Melsh.

Gymnusa Grav.

20.83. brevicollis Grav.

Myllaena Er.

2054. fuscipennis Kroutz.

2085. dubia Er.

2086. minuta Grav.

2087. infuseata Krautz.

Dinopsis Matth.

2088. americanus $K$ raatz.

2089. myllaenoides $K r$ ratz.

\section{Tanygnathus Er.}

2090. collaris Er. 
Acylophorus Nordm.

2091. flavicollis Sachse.

2092. prowus Er.

2093. pratensis Lec.

2094. densus Lec.

2095. flavipes Lec.

Heterothops Steph.

2096. fumigatus Lec.

2096a. fusculus Lec.

2096b. californicus $\mathrm{Lec}$.

2097. pusio Lec.

Quedius Steph.

2098. explanatus Lec.

2099. spelæeus Horn.

2100. fulgidus $F a b$. silvicola Casey.

2101. sublimbatus $M a ̈ k l$.

2102. peregrinus Grav.

2103. capucinus Grav. bardus Melsh.

2104. desertus Horn.

2105. limbifer Horn.

2106. lrvigatus Gyll.

2107. molochinus Grav.

? transparens Mots.

2108. hyperboreus $E r$.

2109. brumnipennis Mann. brunneus err. typ.

2110. debilis Horn.

2111. prostans Horn.

2112. seriatus Horn.

2113. puncticeps Horn.

2114. ferox Lec.

2115. vernix Lec.

Listotrophus Perty.

2116. cingulatus Grav.

2117. capitatus Bland:

\section{Thinopinus Lec.}

2118. pictus Lec.

Creophilus Kirby.

2119. villosus Grav.

Hadrotes Lec.

2120. crassus Mann.
Trigonophorus Nordm.

2121. subcoeruleus Lec.

Xanthopygus Kraatz.

2122. cacti Horn.

\section{Staphylinus Linn.}

2123. nigrellus Horn.

2124. badipes Lec.

2125. pleuralis Lec.

2126. saphyrinus Lec.

2127. luteipes Lec.

2128. erythropterus Linn.

2129. cæsareus Cederh.

2130. vulpinus Nordm.

2131. maculosus Grav.

2132. mysticus Er.

2133. tarsalis Mann.

2134. femoratus Fab. varipes Sachse.

2135. tomentosus Grav.

2136. fossator Grav.

2137. comes Lec.

2138. cinnamopterus Grav.

2139. violaceus Grav.

2140. rutilicauda Horn.

2141. viridans Horn.

2142. exulans Er.

/ 2143. prælongus Mann.

Ocypus Kirby.

2144. ater Grav.

Belonuchus Nordm.

2145. ephippiatus Say.

2146. formosus Grav.

2147. xanthomelas Solsky.

Tympanophorus Nordm.

Euryporus Er. in spec.

2148. puneticollis Er.

Philonthus Curt.

2149. æneus Rossi.

2150. furvus Nord. comptus Hald. ealifornicus Mann.

2151. sericinus Horn. 
2152. nitescens Horn.

2153. alutaceus Horn.

2154. perversus $H o r^{*} n$.

2155. bucephalus Forn.

2156. umbratilis Grav.

2157. laetulus Sity.

2158. gentilis Horn.

2159. asper Horn.

2160 . politus $F a b$.

2161. irinus Horn.

2162. piceatus Sharp.

21(i3. atratus Grav.

2164. semiruber Horn.

2165. basalis Horn.

2166. cautus Er.

2167. hepaticus $E r$.

nanus Melsh.

? varicolor Boh.

2168. gracilicornis Horn.

21169. umbrius Grav.

2170. Havolimbatus $E r$.

2171. pulliatus frav.

2172. pubes Horn.

2173. grandicollis Horn.

2174. puberulus Horn.

2175. quadricollis Horn.

2176. Theveneti Horn.

2177. debilis Grav.

2178. varians Payk. niger Melsh.

2179. Inngicornis Stcph. scybalarius Nord.

2180. discoideus Gruv.

2181. thermarum $A u b e ́$.

2182. alumnus $\mathrm{Er}$.

2183. innocuus Horn.

2181. thoricicus Grav.

2185. fusiformis Melsh.

2186 . fulvipes $F a b$.

21s7. occidentalis Horn.

2188. Schwarzi Horn.

2189. hudsonicus Horn.

2190. Pettiti Horn.

2191. triangulum Horn.

2192. ferreipennis Horn.

2193. caurinus Horn.

2194. Crotehi Horn.

2195. arizonensis Horn.

2196. micans Grav.

2197. Iomatus Er. georgianus Sachse.
2198. eunetans Horn.

2199. xqualis Horn.

2200. brunneus Grav. brevis Melsh. cinctutus Melsh.

2201. cyanipennis $F a b$.

22112. blandus Grav.

2203. quediinus Horn.

2204. sordidus Grav.

2205. cephalotes Grav.

2206. filicornis Horn.

2207 . inquietus Er.

2208 . versutus Horn.

2209. ventralis Grav.

2210. distans Horn.

2211. fallaciosus Horn.

2212. inversus Horn.

2213. brevipennis Horn.

2214. Siegwaldi Mann.

2215. crassulus Horn.

2216. parvus Horn.

2217. clunalis Horn.

2218. bidentatus Horn.

2219. virilis Horn.

2220. punctatellus Horn.

2221. nigritulus Grav,

aterrimus Grav.

picipennis $M \ddot{a} k l$.

2222. microphthalmus Horn.

2223. decipiens Horn.

2224. albionieus Mann.

2225. instabilis Horn.

2226. picicornis Horn.

2227. serpentinus Horn.

2228. baltimorensis Gra:

2229. apicalis Say.

2230. quadrulus Horn.

2231. viridanus Horn.

22:3. confertus Lec.

22:.... Lecontei Horn.

2::3t. aurulentus Horn.

Actobius Steph.

23:3. cinerascens Grav.

22: nanus Horn.

$2: 37$. inutilis Horn.

22:is. fraterculus Horn.

2::39. senilis Horn.

2210 , infimus Horn.

$\because 241$. semipunctatus Fair. \& Ger. 
2212. ocreatus Horn.

2243. sobrinus $E r$.

2244. agnatus Er.

2245. patella Horn.

2246. Ioxatus Horn.

2247. puncticeps Horn.

2248. pusio Horn.

2249. pareus Horn.

2250. gratus Lec.

2251. prderoides Lec.

2252. jocosus Horn.

2253. jucundus Horn.

2254. elegantulus Horn.

2255. terminalis Lec.

2256. umbripennis Lec.

2257. lepidulus Lec.

\section{Cafius Steph.}

2258. canescens Mann.

2259. seminitens Horn.

2260. lithocharinus Lec.

2261. luteipennis Horn.

2262. bistriatus $\mathrm{Er}$.

2263. sulcicollis Lec.

2264. femoralis Mäkl.

2265. sericeus Holme.

2261). decipiens Lec.

2267. opacus Lec.

dubius Lec.

\section{Xantholinus Serv.}

Gyrohypnus Leach.

7 2268. cephalus Say.

2269. fulgidus $F a b$.

2270. obsidianus Melsh.

2271. temporalis Lec.

2272. picipennis Lec.

2273. dimidiatus Lec.

2274. emmesus Grav.

2274a. fusciceps Fauv.

fucosus Casey.

2275. gularis Lec.

2276. obscurus $\mathrm{Er}$.

2277. sanguinipennis Lec.

2278. pusillus Sachse.

2279. hamatus Say.

2280. nanus Lec.

2281. punctulatus Payk.
Leptolinus Kraatz.

2282. rubripennis Lec.

2283. pusio Lec.

Leptacinus Er.

2284. batychrus Gyll. Alavipes Lec.

2285. grandiceps Licc.

2286. brunnescens Lec.

2287. Iongicollis Lec.

2288. parcus Lec.

2289. nigripennis Lec.

2290. ruficollis Lec.

2291. nigritulus Lec.

2292. pallidulus Lec.

2293. seriatus Lcc.

2294. cephalicus Lec.

2295. parumpunctatus Gyll.

Metoponcus Kraatz.

2296. floridanus Lce.

Othius Steph.

2297. californicus Mann.

Baptolinus Kraatz.

2298. macrocephalus Nord.

\section{Diochus Er.}

2299. Schaumii Kraatz.

Dianous Leach.

2300. cærulescens Gyll. chaly beus Lec.

2301. nitidulus Lec. zephyrus Casey.

Stenus Lat.

Areus Casey.

2302. punctiger Casey.

2303. bipunctatus Er. comma Lec.

2304. colon Say.

2305. renifer Lec.

2306. seinicolon Lec.

2307. gemmeus Casey.

2308. bilentigatus Casey. 
2309. sculptilis Casey.

2310. laccophilus Casey.

2311. juno Fab.

2312. verticosus Casey.

2313. tristis Casey.

2314. Austini Casey.

2315. ranops Casey.

2316. delawarensis Casey.

2317. sectator Casey.

2318. zunicus Casey.

2319. femoratus Say.

2320. tacoma Casey.

2321. strangulatus Casey.

2322. tenuis Casey.

2323. angustus Casey.

2324. difficilis Casey.

2325. Pettiti Casey.

2326. scrupeus Casey.

2327. intrusus Casey.

2328. erythropus Melsh.

2329. rugifer Casey.

2330. anastomozans Casey.

2331. shoshonis Casey.

2332. insularis Casey.

2333. vexatus Casey.

2334. Schwarzi Casey.

2335. pauper Casey.

2336. subtilis Casey.

2337. indigens Casey.

2338. simplex Casey.

2339. vicinus Casey.

2340. ingratus Cusey.

2341. neglectus Casey.

2342. scabfosus Casey. i

2343. vespertinus Casey.

2344. convictor Cascy.

2345. deductor Cascy.

confusus \| Cascy.

2346. inornatus Casey.

2347. placidus Casey.

2348. mendax Casey.

2349. eriensis Casey.

2350. sphærops Casey.

2351. militaris Casey.

2352. pluto Casey.

2353. incultus Cusey.

2354. pterobracliys $G$. \& $H$. brevipennis Mikil.

2355. corvus Casey.

2356. maritimus liots.
2:35. montanus Casey.

2358. alpicola Fauv.

2359. costalis Casey.

2360. alveolatus Casey.

2361. elliptieus Casey.

2362. adelops Casey.

236i3. luetuosus Casey.

2364. pusio Casey.

2365. nanulus Casey.

23166. atomarius Casey.

23157. virginia Casey.

23158. texanus Casey.

23ing. venustus Casey.

2370. occidentalis Casey.

2:-71. picificus Casey.

2372. obstrusus Casey.

2:-i: arizonæ Casey.

$2: 3$.4. integer Cascy.

2375. floridanus Casey.

2:36. colonus Er.

2.:37. mammops Casey.

2:37s. gravidus Casey.

2:39!. animatus Casey.

2:5il. tumicollis Cusey.

2:-sil. carinicollis Casey.

2:382. insignis Casey.

2::4:;. stygicus Say.

2.:iᄂ1. egenus Er.

2:४६.;. dolosus Casey.

2initi. villosus Casey.

2:i-7. sectilifer Cuscy.

2ass. milleporus Casey.

2:in!). vinnulus Casev.

2390. congener $\mathbf{M} a k l$.

2:3!1. canaliculatus Gyll.

2:342. carolinæ Crsey.

2:39:3. ageus Casty.

2::!14. pudieus Casey.

2:39.5. minor Cusey.

2::aci. rigidus Casey.

2::17. incertus Cituscy.

2398. parallelus Casey.

2399. noctivagus Casey.

2400. Inculentus Cascy.

2401. juvencus Cascy.

2402. pauperculus Cascy.

24113. haplus Casey.

2404. enodis Cascy.

2405. dives Cusey.

2406. subgriseus Casey. 
2407. terricola Casey.

2408. mundulus Casey.

2409. tuberculatus Casey.

2410. indistinctus Casey.

2411. brumalis Casey.

2412. gratiosus Ca.sey.

2413. monticola Casey.

2414. curtus Casey.

2415. alacer Casey.

2416. hirsutus Casey.

2417. vestalis Casey.

2418. tahoensis Casey.

2419. nimbosus Casey.

2420. pugetensis Casey.

2421. vacuus Casey.

2422. amicus Casey.

2423. exilis Casey.

2424. californicus Casey.

2425. gilin Casey.

2426. dispar Casey.

2427. adspector $M a k l$.

2428. parallelopipedus Mäkl.

2429. cariniceps $M a ̈ k l$.

2430. immarginatus Mäkl.

2431. retrusus Casey.

2432. fraternus Casey.

2433. umbratilis Casey.

2434. croceatus Casey.

2435. advena Casey.

2436. europs Casey.

2437. megalops Casey.

2438. lugens Casey.

2439. plicipennis Casey.

2440. agnatus Casey.

2441. Sayi Casey.

2442. amabilis Casey.

2443. flavicornis $E r$. geniculatus Say.

2444. annularis Er.

2445. perforatus Casey.

2446. nitescens Casey.

2447. pollens Casey.

2448. patens Casey.

2449. reconditus Casey.

2450. propinquus Casey.

2451. canadensis Casey.

2452. pinguis Casey.

2453. utenis Casey.

2454. trajectus Casey.

2455. papagonis Casey.
2456. obsoletus Casey.

2457. artus Crisey.

2458. dilutus Ca:cy.

2459. meridionalis Casey.

2460. callosus Er.

2461. varipes Cisty.

2462. arculus $\mathrm{Er}$.

2463. punctatus Er.

2464. dissentiens Cascy.

2465. Hubbarli Crescy.

2466. lretulus Crescy.

2467. leviceps Cisscy.

2468. politulus Cusey.

2469. simiolus Cascy.

2470. pertinax Casey.

2471. Iucidus Casey.

- chalybieus Boh.

\section{Euæsthetus Grav.}

2472. brevipennis Crsey.

2473. punctatus Casey.

2474. florida Cascy.

2475. similis Cavey.

2476. mundulus Cascy.

2477. robustulus Cascy.

2478. iripennis Cascy.

2479. texanus Cuscy.

2480. americanus $E r$.

2481. atomus Cascy.

Edaphus Lec.

2482. nitidus Lec. luculentus Casey. carinatus Casey.

\section{Stictocranius Lec.}

2483. puncticeps Lec.

\section{Cryptobium Mann.}

2484. badium Grav.

2485. floridunum Lec. convergens Casey. parallelum Casey. proximum Casey.

2486. anceps ITorn.

2487. despectum Lec.

2488. lugubre Lec.

2489. obliquum Lec. parcum Lec. 
2490, bicolor Grav.

2490a. melanocephalum Er.

2491. carolinum $\mathrm{Er}$.

2492. texanum Lec.

2493. Lecontei Horn.

2494. vagum Horn.

2495. pimerianum Lec.

2496. arizonense Horn.

2497. vitatum Horn.

2498. ventrale Horn.

2499. prospiciens Lec.

2500. tumidum Lec.

2501. californicum Lec.

2502. pallipes Grav. capito Casey.

2502a. latebricola Nordm.

2503. flavicorne Lec.

2504. properum Horn.

2505. pusillum Lec.

2506. lepidum Lec.

2507. cribratum Lec.

2508. sellatium Lec.

2509. serpentinum Lec.

\section{Ababactus Sharp.}

2510. nactus Horn.

\section{Lathrobium Grav.}

2511. grande Lec.

2512. punctulatum Lec.

2513. angulare Lec.

2514. nigrum Lec.

2515. bicolor Lec.

2516. nitidulum Lec.

2517. finitimum Lec.

2518. puncticeps Lec.

2519. jacobinum Lec.

2520. subseriatum Lee.

2521. brevipenne $L c c$.

2522. armatum Say.

2523. othioides Lec. inops Casey.

2524. simile Lec.

2525. coneolor Ler:

2526. simplex Lec.

2527. tenue Lec.

2525. divisum Lec.

2529. pedale $L c c$.

25311. debile Lec.

2531. politum Grav.
2532. confusum Lec.

2533. seriatum Lec.

2534. longiusculum Grav.

2535. pareum Lec.

2536. californieum Lec.

2537. collare $\mathrm{Er}$.

2538. ambignum Lec.

2539. ventrale Lec. tricolor Casey.

2540. anale Lcc.

2541. pallidulum Lec.

2542. lituarium Lec.

2543. dimidiatum Say.

__ puncticolle Kirby.

Scopæus Er. Orus Casey.

2544. opacus Lec.

2545. nitidus Lec.

2546. exiguus $\mathrm{Er}$.

2547. picipes Casey.

2548. dentiger Lec.

2549. brunnipes $L c c$.

2550. punctatus Casey.

\section{Stilicus Lat.}

Rugilus Leach.

25i1. tristis Mrclsh.

2.52. quadriceps Lec.

255\%. opaculus Lec.

2.5.5. angularis Lec.

295.5. latiusculus Casey.

25iti. dentatus Say.

2.isi. biarmatus Lec.

2.5. . rudis Lec.

\section{Lithocharis Er.}

2559. corticina Grav.

25b0. confluens Say.

2561. ochracea Grav.

2562. obsoleta Nordm.

2563. tabacina Casey.

Dacnochilus Lec.

2564. angularis $E r$.

\section{Liparocephalus Mäkl.}

2565. brevipennis Mrikl.

2566. cordicollis Lec. 


\section{Paderus Grav.}

2567. femoralis Lec.

2568. grandis Aust.

2569. mexicanus Er.

25\%0. littoreus Aust.

2571. compotens Lec.

2572. palustris Aust.

y 2573. littorarius Grav.

2574. floridanus Aust.

2575. obliterutus Lec.

2576. ustus Lec.

\section{Sunius Steph.}

2577. californicus Aust.

2578. prolixus $E r$.

2579. binotatus Say. linearis $E r$.

2581. brevipennis Aust.

2581. longiuseulus Mann.

Stilicopsis Sachse.

2582. paradoxa Sachse.

2583. monstrosa Lec.

\section{Pinophilus Grav.}

2584. picipes Er.

2585. latipes Grav.

2586. parcus Lec.

2587. densus Lec.

2588. opacus Lec.

\section{Palaminus Er.}

2589. pallipes Lec.

2590. flavipennis Lec.

2591. normalis Lec.

2502. testacens Er.

2593. contortus Lec.

2594. lividus Lec.

2595. cribratus Lee.

2596. pumilis Lec.

2597. larvalis Lec.

\section{Hypocyptus Mann.}

2598. longicornis Payk.

2599. Crotchii Horn.

2600. nigritulus Lee.

Microcyptus Horn.

Anacyptus Horn.

2601. testaceus Lec.
Trichopsenius Horn.

2602. depressus Lec.

Xenistusa Lec.

2603. cavernosa Lec.

2604. fossata Lec.

2605. pressa Lec.

\section{Tachinus Grav.}

2606. maculicollis Mäkl.

2607. semirufus Horn.

2608. memnonius Grav.

2609. tachyporoides Horn.

2610. agilis Horn.

2611. angustatus Horn.

2612. debilis Horn.

2613. repandus Horn.

2614. serutator Horn.

2615. mimus Horn.

2616. addendus Horn.

2617. parallelus Horn.

2618. nigricornis Mann.

2619. flavipennis $D e j$. luridus $\ddagger$ Horn.

2620. luridus $E r$. canadensis Horn.

2621. fimbriatus Grav. ? puncticollis Ziegl.

2622. picipes $E r$.

2623. Schwarzi Horn.

2624. limbatus Melsh.

2625. fumipennis Say.

2626. pallipes Grav. frigidus $E r$.

2627. Crotchii Horn.

2628. instabilis Mäkl.

2629. circumeinctus Mïkl.

2630. nitiduloides Horn.

2631. elongatus Gyll.

\section{Tachyporus Grav.}

2632. maculipennis Lec.

2633. elegans Horn.

2034. jocosus Say.

2635. chrysomelinus Linn.

2636. californicus Horn.

2637. nanus Er. 
2638. seitulus Er.

2639. brumeus Fab.

\section{Cilea Duval.}

2640. silpluoides $\operatorname{Linn}$.

\section{Physetoporus Horn.}

2641. grossulus Lcc.

Erchomus Mots.

2642. convexus $E r$. inllitus Horn.

264?. punctipennis Lec.

2644. ventriculus Say. aflinis finby. llavidus Carey.

2645. Jnevis Lec.

\section{Conosoma Iraatz.}

2646. littoremm Linn.

2647. Kuoxii Lec.

2648. crissum Grav. versicolor Casey.

2649. bisignatum Horn.

2650. cistaneun Horn.

2651. pubescens Payk. imbricatus Casey.

2652. parvulum Horn.

2653. basale $\mathrm{Er}$. occultus Cascy.

2654. opieum Say. corticola Casey. setifer Cascy.

2655. seriptum Horn.

Boletobius Leach.

2656. niger Grav.

2657. axillaris Grav. facilis Cuxey.

2658. dimidiatus $\mathrm{Er}$.

2659. cingulatus Mann.

2660. intrusus Horn.

2661. cineticullis Say. occiduus Casey.

2662. anticus Horn.

2663. pygmrus Fab. angularis Sachse.

2664. trinotatus Er.
2665. obsoletus Say.

\& 2666. cinctus Grav.

2666a. gentilis Lec.

2667. longiceps Lec.

26ik8. quæsitor Horn.

\section{Bryoporus Kraatz.}

2669. flavipes Lec.

2670. rufescens Lec. parvulus Casey.

Mycetoporus Mann.

2671. humidus Say. lepidus $\ddagger$ Horn.

2672. tenuis Horn.

2673. consors Lec.

2674. amerieanus $\mathrm{Er}$. lucidulus Lec.

2675. flavicollis $L e c$.

2676. splendidus Grav. americanus \pm Horn. inquisitus Casey.

2677. insignis Makl.

2678. nigrans Makl.

Habrocerus Er.

2679. Schwarzii Horn.

2680. magnus Lec.

Olisthærus Heer.

2681. megacephalus Zett.

2682. substriatus Gyll.

Pseudopsis Newm.

2683. columbica Faur.

2684. obliterata Lec.

Megalops Er.

2685. cælatus Grov.

2686. rufipes $L e c$.

Oxyporus Fab.

1'2687. femoralis Grav. 2688. austrinus Horn.

2689. elegans Lec.

2690. major Grav.

2691. rufipennis Lec.

2692. stygicus Say. 
2693. vittatus Grav.

2694. fasciatus Melsh.

2695. bicolor Fauv.

2696. lateralis Grav.

2697. occipitalis Fauv.

2698. lepidus Lec.

2699. 5-maculatus Lec.

\section{Osorius Lat.}

2700. politus Lec.

2701. planifrons Lec.

2702. latipes Grav.

\section{Holotrochus Er.}

2703. minor Fauv.

2704. lævicauda Lec.

\section{Bledius Leach.}

2705. gularis Lec.

2706. pallipennis $E r$.

2707. fortis Lec.

2708. mandibularis $\mathrm{Er}$.

2709. brevidens Lec.

2710. ferratus $L e c$.

2711. jacobinus Lec.

2712. armatus $E r$.

2713. cribricollis Lec.

2714. cuspidatus Lec.

2715. politus Er.

2716. flavipennis Lec.

2717. semiferrugineus Lec.

2718. rotundicollis $L e c$.

2719. fumatus Lec.

2720. nitidiceps Lec.

2721. opacifrons Lec.

2722. rubiginosus $E r$.

2723. analis $L e c$.

2724. nitidicollis Lec.

2725. punctatissimus Lec.

2726. laticollis Lec.

2727. longipennis Makl.

2728. luteipennis $L e c$.

2729. sinuatus Lec.

2730. annularis Lee.

2731. confusus Lec.

2732. ruficornis Lee.

2733. divisus Lec.

2734. pleuralis Lec.

2735. diagonalis Lec.
2736. tau Lec.

2737. ornatus Lec.

2738. suturalis Lec.

2739. phytosinus Lec.

2740. cognatus Lec.

2741. emarginatus Say.

2742. basalis Lec.

2743. dimidiatus Lec.

2744. opaculus Lec.

2745. cordatus Say.

2746. forcipatus Lec.

_- ? albonotatus $M a ̈ k l$.

__ ? fasciatus Say.

__ ? melanocephalus Say.

\section{Platystethus Mann.}

L) 2747. americanus Er.

\section{Oxytelus Grav.}

2748. incolumis Er.

2749. sculptus Grav.

2750. rugosus Grav.

2751. niger Lec.

2752. pennsylvanicus $\mathrm{Er}$.

2753. fuscipennis Mann.

2754. convergens Lec.

2755. insignitus Grav.

2756. punctatus $L e c$.

2757. nitidulus Grav.

2758. sobrinus Lec.

2759. placusinus Lec.

2760. depressus Grav.

2761. nanus $E r$.

2762. exiguus $E r$.

Haploderus Steph.

Phloeonaeus Er.

2763. linearis Lec.

2764. laticollis Lec.

\section{Trogophlœus Mann.}

276.5. simplarius Lec.

2766. convexulus Lec.

2767. blediinus Lec.

2768. uniformis Lec.

2769. lithocharinus $L e c$.

2770. arcifer Lec.

2771. caloderinus $L \ell c$. 
2772. phlooporinus Lec.

2773. 4-punctatus Say.

2774. subtilis $E r$.

2775. fulvipes $E r$.

2776. memnonius Er.

Apocellus Er.

2777. stilicoides Lec.

2778. analis Lec.

2779. brevipennis Casey.

2780. gracilicornis Casey.

2781. crassicornis Casey.

2782. sphæricollis Say.

2783. bicolor Casey.

\section{Ancyrophorus Kraatz.}

2784. planus Lec.

2785. biimpressus Mäkl.

Thinobius Kies.

2786. oxytelinus Lec.

2787. flavicornis Lec.

2788. brachypterus Lec.

2789. fimbriatus Lec.

2790. macropterus Lec.

2791. antennarius Fauv.

\section{Syntomium Er.}

2792. confragosum Midkl.

Zalobius Lec.

2793. spinicollis Lec.

2794. serricollis Lec.

\section{Coprophilus Er.}

2795. striatulus $F a b$.

\section{Deleaster Er.}

2796. concolor Lec.

\section{Porrhodites Kraatz.}

2797. fenestralis Zett.

\section{Geodromicus Redt.}

2798. cresus $E r$.

2799. brunneus Say.

2800. nigrita Müll.

2801. ovipennis Lec.
Tilea Fauv.

2802. cavicollis Fauv.

\section{Lesteva Lat.}

2803. pallipes Lec.

2804. fusconigra Mots.

Acidota Steph.

2805. crenata Fab.

2806. subcarinata $\mathrm{Er}$.

2807. quadrata Zett.

\section{Arpedium Er.}

2808. cribratum Fauv.

2809. Schwarzii Fruv.

2810. angulare Fauv.

2811. tenue Lec.

2811a. brunnescens Sahlb.

Amphichroum Kraatz.

2812. testaceum Mann.

2813. floribundum Lec.

2814. lævieolle Lec.

2815. sparsum Fauv.

2816. sentatum Fanv.

2817. maculatum Lec.

2818. puberulum Fanv.

2819. opaculum Fauv.

Tanyrhinus Mann.

2820. singularis Mann.

Trigonodemus Lec.

2821. striatus Lec.

\section{Lathrimæum Er.}

2422. sordidum $E r$.

2823. subcostatum Miikl.

2824. fimetarium $M$ Cakl.

2825. pistum Fauv.

Deliphrum Er.

2826. expansum Lec.

Orochares Kraatz.

2827. angustatus $\mathrm{Er}$. 


\section{Olophrum Er.}

2828. obtectum Er.

2829. marginatum Kirby.

2830. Convexum Mïhl.

2831. rotundicolle Sahlb.

2832. parvulum Mäkl.

2833. latum $\mathrm{Makk}$.

\section{Pycnoglypta Thom.}

2834. lurida Gyll.

Acrulia Thom.

2835. tumidula Mikl.

\section{Micralymma Westw.}

2836. Stimpsonii Lec.

2837. brevilingue Schiödte.

\section{Homalium Grav.}

2838. strigipenne $M a ̈ k l$.

2839. longulum $M a ̈ k l$.

2840. lapponicum Zett.

2841. pusillum Grav.

2842. repandum $\mathrm{Er}$.

2843. foraminosum Mäkl.

2844. plagiatum Mann.

2845. exculptum Mäkl.

2846. callosum Mäkl.

2847. humile Mäkl.

2848. flavipenne $M a ̈ k l$.

2849. diffusum Fauv.

2850. megarthroides Fauv.

2851. hamatum Fauv.

2852. humerosum Fauv.

2853. punctiventre Fauv.

2854. rufipes Fauv.

2855. fractum Fauv.

2856. texanum Fouv.

2857. alutaceum Fauv.

2858. cribrum Fauv.

2859. Theveneti Fauv.

2860. rivulare Payk.

\section{Anthobium Leach.}

2861. fimetarium $E r$.

2862. sorbi Gyll.

2863. pothos Mann.

2864. rigulosum Mäkl.
2865. californicum Fauv.

2866, segmentarium $M$ iik $k l$.

2867. marginatum Say.

2868. convexum Fauv.

2869. aurifluum Fauv.

2870. Hornii Fauv.

Orobanus Lec.

2871. simulator Lec.

Micrædus Lec.

2872. laticollis Mann.

2873. Austinianus $L \subset c$.

Ephelis Fauv.

2874. guttata Lec.

2875. pallida $L e c$.

2876. notata Lec.

Eudectus Redt.

2877. crassicornis Lec.

\section{Protinus Lat.}

2878. limbatus Miki.

2878a. Mäklini Fauv.

2879. atomarius $\mathrm{Er}$.

2880. basalis Mrikl.

2881. sulcatus Fauv.

\section{Megarthrus Steph.}

2882. pietus Mots.

2883. americanus Sachsc.

2884. excisus $L e c$.

2885. sinuaticollis Lac. angulicollis Mikkl.

2886. atratus $M a ̈ k l$.

\section{Lispinus Er.}

Ancceus Fauv.

2887. exiguus $\mathrm{Er}$.

2888. prolixus Lec.

2889. ealifornicus Lec.

2890. æquipunctatus Lec.

2891. linearis $E r$.

2892. tenellus Er.

\section{Glyptoma Er.}

2893. costale Er.

2894. brevicristatum Horn. 
Triga Fauv.

2895. picipennis Lec.

Eleusis Lap.

2896. pallidus Lec.

2897. fasciatus Lec.

2898. nigrellus Lec.

Siagonium Kirby.

2899. americanum Melsh.

2900. punctatum Lec.

Hypotelus Er.

2901. capito Lec.

Trigonurus Muls.

2902. rugosus Sharp.
2903. cælatus Lec. Leconteus Sharp.

2904. Edwardsii Sharp.

2905. Crotehii Lec.

Micropeplus Lat.

2906. laticollis Mäkl.

2907. punctatus Lec.

2908. cribratus Lec.

2909. sculptus Lec.

2910. obliquus Lec.

2911. tesserula Curt.

2912. brunneus Mäkl.

Kalissus Lec.

2913. nitidus Lec.

\section{TRICHOP'T EIR Y GIDA.}

Nossidium Er.

2914. americanum Mots.

2915. posthumum Matth.

Nanosella Mots.

2916. fungi Mots

Actidium Matth.

2917. Crotchianum Matth.

2918. politum Matth.

Motschulskium Matth.

2919. sinuatocolle Matth.

Micridium Mots.

Ptilium Er.

2920. Sharpi Matth.

2921. columbianum Matth.

2922. humile Matth.

2923. obscurum Matth.

2924. fungi $L e c$.

2925. Hornianum Matth. fungicola Casey.

2926. Collani Mäkl.

_- ? truncatum Casey.
Ptenidium Er.

2927. Ulkei Matth.

2928. speculifer Matlh.

2929. evanescens Marsh.

2930. atomaroides Mots.

2931. pullum Mäkl.

2932. Mannerheimii Matth.

2933. foveicolle Lec.

2934. ? lineatum Lec.

- glabratum Mots.

__ macrocephalum Nietn.

Limulodes Matth.

2935. paradoxus Matth.

Pteryx Matth.

2936. brunnea Lec.

2937. balteata Lec.

2938. Duvalii Matth.

2939. perforita Mots.

Ptinellodes Matth.

2940. Lecontei Hath. G\%H

Trichopteryx Kirby.

2941. Aubói Matth.

2942. morens Matth. 
2943. ambigua Matth.

2944. aspera Hald.

2945. parallela Mots. longipennis Casey.

2946. vicina Matth.

2947. castanea Matth.

2948. sericans Heer.

2949. californica Matth.

2950. Hornii Matth.

2951. xanthocera Matth.

2952. parallelopipeda Matth.

2953. diffinis Matth.

2954. Josephi Matth.

2955. fascicularis Hbst.

2956. cognata Matth.

2957. abrupta Hald.

2958. Henrici Matth.

2959. sitkaensis Mots.

? insularis $M a ̈ k l$.

2960. crassicollis Mükl.

2961. laticollis Mann.
2962. atomaria $\mathrm{DeG}$.

2963. discolor Hald.

funginus Casey.

2964. Dohrnii Math.

2965. glabricollis Matth.

2966. Haldemanni Lec.

2967. Schaumii Matth.

2968. fuscipennis Hald.

_._ radicola Casey.

Smicrus Matth.

2969. filicornis Fairm.

Ptinella Mots.

2970. quercus Lec.

2971. pini Lec.

Nephanes Thom.

2972. læviusculus Matth.

\section{H Y DROSCA PIID $\mathrm{A}$.}

Hydroscapha Lec.

2973. natans Lec.

\section{SPIARIIDE.}

Sphærius Waltl.

Microsporus Kol.

2974. politus Horn.

\section{SCA PII DIID E.}

Scaphidium Oliv.

2975. quadriguttatum $S a y$.

3975a. obliteratum Lec.

2975b. quadripustulatum Say.

2975c. piceum Melsh.

Scaphium Kirby.

2976. castanipes Kirby.

\section{Cyparium Er.}

2977. flavipes Lec.

Broocera Er.

2978. concolor $F a b$.

2979 apicalis (ec)
Scaphisoma Leach.

2079. apiealis Lec.

2980. castaneum Mots.

2981. convexum Say.

2982. punctulatum Lec.

2983. suturale Lec.

2984. terminatum Melsh.

2985. rufulum $L e c$.

2986. pusillum Lec.

\section{Toxidium Lec.}

2987. gammaroides Lec.

2988. compressum Zimm. 
PHA LACIEA.

Phalacrus Payk.

2989. seriatus Lec.

2990. ovalis Lec.

2991. dispar Lec.

2992. penicillatus Say.

2993. politus Melsh.

2994. pumilio Lcc.

2995. simplex Lec.

Olibrus Er.

2996. vittatus Lec.

2996a. nigricollis Lee.

2997. bicolor Gyll.

2998. striatulus $L e c$.
2999. rufipes Lec.

3000, semistriatus Lec.

3001. rubens $L e c$.

3002. pallipes Say.

3003. obtusus Lec.

3004. princeps $S z$.

3005. consimilis Marsh.

3006. aquatilis Lec.

73007 . nitidus Melsh.

3008. pusillus Lec.

Litochrus Er.

3009. pulchellus $L e c$.

3010. immaculatus Zimm.

\section{COR Y 1,O IPIH ID. E.}

Sacium Lec.

3011. lugubre Lec.

3012. obscurum Lec.

3013. biguttatum Lec.

3014. amabile Lec.

3015. fasciatum Say.

3016. lepidum Lec.

3017. lunatum Lec.

3018. decolor Lec.

3019. misellum Lec.

3020. scitulum Lec.

3021. mollinum $S z$.

3022. splendens $S z$.

Arthrolips Woll.

3023. marginicollis Lee.
Corylophus Steph.

3024. truneatus Lee.

\section{Sericoderus Steph.}

3025. flavidus Lec.

3026. obscurus Lec.

3027. subtilis $L c c$.

Rhypobius Lec.

3028. marinus Lec.

Orthoperus Steph.

3029. glaber Lec.

3030. elongatus Lec.

3031. suturalis Lec.

3032. scutellaris $L e c$.

\section{COCOINELIID.E.}

Anisosticta Duponehel.

3033. strigata Thunb.

3034. seriata Melsh.

\section{Næmia Muls.}

3035. epjscopalis Kirby.

Megilla Muls.

3036. maculata $D e G$.

3037. vittigera Mann.
Ceratomegilla Cr.

3038. Ulkei $\mathrm{Cr}$.

Adonia Muls.

3039. constellata Laich.

Eriopis Muls.

3040. connexa Germ.

Hippodamia Muls.

3041. 5-signata Kirby.

7 3042. ambigua Lec. 
3043. Lecontei Muls.

3043a. mosta Lec.

:044. glacialis Fab.

3045. 15-maculata Muls.

(5) 3046. convergens Guér. modesta Melsh.

3047. spuria Lec.

3048. oregonensis $\mathrm{Cr}$.

3049. sinuata Muls.

3050. 13-punctata Linn.

3051. parenthesis Say.

3052. falcigera $\mathrm{Cr}$.

3053. americana $\mathrm{Cr}$.

3054. variegata Goeze.

\section{Coccinella Linn.}

Cycloneda $\mathrm{Cr}$.

3055. affinis Rand.

$3655 \%$. venusta Melsh.

3056. trifasciata Linn.

3056a. juliana Muls.

3056b. subversa $\mathrm{Lec}$.

3057. difficilis $\mathrm{Cr}$.

$\&$ 3058. 9-notata Host.

$3058 a$. franciscana Muls.

$\&$ 3059. transversoguttata Fab. 5-notata Kirby.

3059a. transversalis Muls.

3059b. californica Mann.

3060. monticola Muls.

3061. prolongata $\mathrm{Cr}$.

3062. tricuspis Kirby.

3063. annectans $\mathrm{Cr}$.

$\hat{\gamma}$ 3064. sanguinea Linn.

3065. oculata Fab.

y 3065a. abdominalis Say.

_._. Menetriesi Muls.

Adalia Muls.

خे 3066. frigida Schn.

\& 3067. bipunetata Linn.

3068. ? ludovicæ Muls.

Harmonia Muls.

Anisocalvia Cr.

\& 3069. picta $R$ and.

3070. cyanoptera Muls.

3071. 14-guttata Linn. 3071a. similis Rand.

3071b. hesperica $C r$.

3072. 12-maculata Gebl.

Mysia Muls.

3073. pullata Say.

3074. Hornii $\mathrm{Cr}$.

Anatis Muls.

4 3075. 15-punctata Oliv.

3076. Rathvoni Lec.

3077. . subvittata Muls.

Psyllobora Muls.

$\&$ 3078. 20-maculata Say.

$3078 \alpha$. tædata Lec.

3079. nana Muls.

Chilocorus Leach.

\$ 3080. bivulnerus Muls.

A 3081. cacti Linn.

\section{Exochomus Redt.}

:1152, Pilatii Muls.

3083. marginipennis Lec.

3083a. æthiops Bland.

3084. contristatus Muls.

3085. bipustulatus Dej.

:3086. tripustulatus $D e G$.

Cryptognatha Muls.

3087. pallida Lec.

3088. pusilla Lee.

Pentilia Muls.

3089. marginata Lec.

3090. misella Lec.

3091. coccidivora Ashm.

3092. ovalis Lec.

Brachyacantha Chev.

3093. lepida Muls.

3094. dentipes $F a b$.

3094a. tau Lec.

3094b. quadrillum Lec.

3095. ursina Fab.

(4) 3095a.10-pustulata, Melsh. 
3095b. flavifrons Muls. 3095c. basalis Melsh. 3095d. albifrons Say. 3096. 4-punctara Melsh. 3097. indubitabilis $\mathrm{Cr}$. 3098. Bollii Cr. 3099. querceti $S z$. - ? felina $F a b$.

\section{Hyperaspis Chev.}

3100. Bolteri Lec.

3101. fimbriolata Melsh.

3101a. dissoluta $\mathrm{Cr}$.

\& 3102. lateralis Muls. Hornii $\mathrm{Cr}$.

3103. tæniata Lec.

3104. cruenta Lec.

$\Leftrightarrow$ 3105. undulata Say.

3106. disconotata Muls.

3107. discreta Lec.

3108. Lewisii $\mathrm{Cr}$.

3109. tredata Lec.

3110. signata Oliv.

3111. osculans Lec.

$\theta$ 3112. proba say.

3113. gemina Lec.

3114. bigeminata Rand.

3115. pratensis Lec.

3116. lugubris Rand.

311 iba. venustula $M u l s$.

y 3117. 4-oculata Mots.

3118. postica Lec.

3119. paludicola $\mathbf{S z}$.

3120. punctata $L e c$.

3121. annexa Lec.

3122. 4-vittata Lec.

3123. morens Lec.

3124. tristis Lec.

3125. inedita Muls.

\section{Hyperaspidius Cr.}

3126. trimaculatus Linn.

3127. militaris Lec.

3128. areuatus tec.

\section{Seymnus Kug.}

3129. amabilis Lee.

3130. ornatus Tec.

3131. myrmidon Muls.

3132. guttulatus Lec.
3133. flavifrons Melsh.

3134. bioculatus Muls.

3135. terminatus Say.

3136. xanthaspis Muls.

3137. femoralis Lec.

3138. americanus Muls.

3139. nebulosus Lec.

3140. pallens lec.

3141. debilis Lec.

3142. cinctus Lec.

3143. Lecontei $\mathrm{Cr}$. suturalis \| Lec.

3144. arcuatus Rossi.

3145. fraternus Lec.

` 3146. hæmorrhous Lec.

3147. Brullei Muls.

3148. collaris Melsh.

3149. caudalis Lec.

3150. consobrinus Lec.

3151. fastigiatus Muls.

3152. puncticollis Lec.

3153. cervicalis Muls.

3154. socer Lec.

3155. marginicollis Mann.

3156. tenebrosus Muls.

3157. lacustris Lec.

3158. abbreviatus Lec.

3159. nanus Lec.

3160. punctum Lec.

3161. icteratus MIuls.

3162. punctatus Melsh.

3163. baltentus Lec.

3161. quadritæniatus Lec.

3165. nigripennis Lec.

3166. coniferarum $\mathrm{Cr}$.

3167. pacificus $\mathrm{Cr}$.

3168. Phelpsii $\mathrm{Cr}$.

--_ ? cyanescens Muls.

\section{Cephaloscymnus $\mathrm{Cr}$.}

3169. Zimmermanni $\mathrm{Cr}$.

\section{Coccidula Kug.}

3170. lepida Lec.

\section{Epilachna Chev.}

3171. borealis $F a b$.

3172. mexicana Guér.

3173. corrupta Muls. 


\section{ENDOM Y CHIDA.}

Alexia Steph.

3174. Jobata Lec.

Anamorphus Lec.

Symbiotes Redt.

3175. Ulkei Cr.

3176. minor $\mathrm{Cr}$.

Mycetæa Steph.

3177. hirta Marsh.

\section{Rhanis Lec.}

3178. unicolor Ziegl.

Liestes Redt.

Phymaphora Newm.

3179. pulchella Newm.

3180. californica Horn.

Iycoperdina Lat.

3181. ferruginea Lec.

Xenomycetes Horn.

3182. Morrisoni Horn.
Aphorista Gorh.

3183. morosa Lec.

3184. pallida Horn.

3185. læta Lec.

3186. vittata $F a b$.

Mycetina Muls.

3187. perpulchra Nerom.

3188. Hornii $\mathrm{Cr}$.

3189. testacea Ziegl.

3190. limbata Horn.

Stenotarsus Perty.

3191. hispidus Hbst.

Epipocus Germ.

3192. cinctus Lec.

3193. mutilatus Gerst.

3194. punctatus Lec.

3195. discoidalis Le.c.

3196. bivittatus Gerst.

3197. unicolor Horn.

Endomychus Panz.

3198. biguttatus Say.

\section{ERATYLIDAE.}

\section{Languria Lat.}

3199. bicolor $F a b$.

3200. Mozardi Lat.

3201. discoidea Lee.

3202. tædata Lec.

3203. marginipennis $\mathbf{S z}$.

3204. angustata Beauv.

$3204 a$. trifasciata Say.

3204b. Uhlerii Horn.

3205. convexicollis Horn.

c 3206. læta Lec.

3207. Lecontei $\mathrm{Cr}$.

3208. collaris Lec.

3209. gracilis Nerom.

$3209 a$. inornata $R$ and.

3210. divisa Horn.

_._. femoralis Mots.
Langurites Mots.

3211. lineatus Lap.

Plœosoma Woll.

Hypodacne Lec.

3212. punctata Lec.

Dacne Lat.

3213. 4-maculata Say.

3214. picea Lec.

3215. californica Horn.

Megalodacne Cr.

$>3216$. fasciata $F a b$.

3217. heros Say.

321๙. Ulkei Cr. 
Ischyrus Lac.

3219. 4-punctatus Oliv.

3220. nigrans $C r$.

3221. extricatus $\mathrm{Cr}$.

\section{Mycotretus Lac.}

3222. sanguinipennis Say.

3223. pulchra Say.

3223a. dimidiata Lac.

3224. dissimulator $C_{r}$.

Tritoma Fab.

Cyrtotriplax Cr.

Triplax Hbst.

3225. humeralis $F a b$.

$3225 a$. ruficeps Lec.

3225b. vittata Lec.

3225c. aulica Horn.
Q 3226. biguttata Say.

3227. mimetica $C r$.

3228. erythrocephala $\mathrm{Lac}$.

3229. atriventris Lec.

3230. angulata Say.

3231. unicolor Say.

3232. affinis $L a c$.

3233. frontalis Horn.

3234. festiva fec. $\mathcal{L}$ ac

3235. macra Lec.

3236. thoracica Say.

3237. antica Lec.

3238. californica Lec.

3239. flavicollis Lac.

\section{Erotylus Fab.}

Cypherotylus Cr.

3240. Boisduvali Chev.

\section{COLYDIIDE.}

\section{Rhagodera Er.}

3241. tubereulata Mann. 3242. costata Horn.

Anchomma Lec.

3243. costatum Lec.

Synchita Hellw.

3244. laticollis Lec.

3245. obscura Horn.

3246. variegata Lec.

3247. parvula Guér.

3248. fuliginosa Melsh.

3249. granulata Say.

3250. dentata Horn.

\section{Cicones Curt.}

3251. marginalis Melsh.

3252. lineaticollis Horn.

\section{Ditoma Ill.}

3253. carinata Lec.

3254. quadriguttata Say.

3255. quadricollis Horn.

3256. ornata Lec.

3257. sulcata Lec.
Eudesma Lec.

3258. undulata Melsh.

\section{Endophlœus Er.}

3259. nosodermoides Horn.

\section{Phlœonemus Er.}

3260. catenulatus Horn.

\section{Coxelus Lat.}

3261. guttulatus Lec.

3262. pacificus Horn.

3263. serratus Horn.

\section{Lasconotus Er.}

3264. complex Lec.

3265. borealis Horn.

3266. linearis $\mathrm{Cr}$.

3267. vegrandis Horn.

3268. simplex Lec.

3269. servus Horn.

3270. referendarius Zimm.

3271. pusillus Lec.

\section{Aulonium Er.}

3272. parallelopipedum Say. æequicolle Lec. 
3273. longum Lec.

3274. tuberculatum Lec.

3275. ferrugineum Zimm.

Colydium Fab.

3276. lineola Say. nigripenne Lec.

-_ ? longiusculum Say.

\section{Nematidium Er.}

3277. mustela Pasc.

Aglenus Er.

3278. brunneus Gyll.

Oxylæmus Er.

3279. americanus Lec.

3280. californicus $\mathrm{Cr}$.

\section{Deretaphrus Er.}

3281. oregonensis Horn.

\section{Sosylus Er.}

3282. costatus Lec.

3283. dentiger Horn.

Penthelispa Pasc.

3284. hæmatodes $F a b$.
3285. reflexa Say. nitida Lec.

Pycnomerus Er.

3286. sulcicollis Lec.

Bothrideres Er.

3287. geminatus Say.

3288. montanus Horn.

Erotylathris Mots.

Machlotes Pase.

3289. exaratus Melsh.

Cerylon Lat.

3290. castaneum Say. unicolor Ziegl.

Philothermus Aubé.

3291. glabriculus Lec.

3292. puberulus $S z$.

Murmidius Leach.

3293. ovalis Beck.

Mychocerus Er.

3294. depressus Lec.

\section{RH YSSO DI D $\mathrm{E}$.}

Rhyssodes Dalm.

3295. exaratus $\mathrm{Ill}$.

3296. hamatus Lec.
Clinidium Kirby.

3297. sculptile Newm. conjungens Germ.

3298. calcaratum Lec.

\section{CUCUJIDA.}

Silvanus Lat.

3299. surinamensis Linn.

3299a. bicornis $E r$.

3300. bidentatus $F a b$.

3301. planatus Germ. nitidulus Lec.

3302. imbellis Lec.

3303. cassiæ Reiche. quadricollis $\ddagger$ Lec.

3304. gilie Casey.

3305. advena Waltl.

3306. rectus Lec.
3307. opaculus Lec.

Nausibius Redt.

3308. dentatus Marsh.

3309. repandus Lec.

Catogenus Westw.

3310. rufus $F a b$.

Scalidia Er.

Taphroscelidia $\mathrm{Cr}$.

3311. linearis Lec. 
Prostomis Lat.

3312. mandibularis Fab. americana $\mathrm{Cr}$.

Narthecius Lec.

3313. grandiceps Lec.

Pediacus Shuck.

3314. fuscus $\mathrm{Er}$. subcarinatus Mann.

3315. depressus Hbst.

3315a. subglaber Lec.

Cucujus Fab.

Y 3316. clavipes Fab.

$3316 a$. puniceus Mann.

\section{Ino Lap.}

3317. reclusa Lec.

3318. immunda Reit.

\section{Læmophlæus Lap.}

3319. terminalis Casey.

3320. biguttatus Say.

3321. fasciatus $\mathrm{Mr}$ lsh.

3322. Lecontei Grour.

3323. floridanus Casey.

3324. Chæmeropis $S z$.

3325. modestus Say.

3326. convexulus Lec.

3327. adustus Lec.

7 3328. testaceus Fab.

3329. nitens Lec.

Gundlachi Grouv.

3330. punctatus Lec.

3...:1 Horni Casey.

3332. rotundicollis Casey.

3333. quadratus Casey.

3334. cephalotes Lec.

:335. angustulus Lec.

:336. Schwarzi Casey.
3337. extricatus Casey.

3338. alternans $E r$.

3339. ferrugineus Steph.

3340. pubescens Casey.

3341. truncatus Casey.

3342. pusillus Sch. longicornis Mann.

3343. denticornis Casey.

-_ pusillimus Mann.

- Reitteri Grouv.

\section{Lathropus Er.}

3344. vernalis Lec.

3345. pictus $S z$.

3346. pubescens Casey.

Dysmerus Casey.

3347. basalis Casey.

Dendrophagus Sch.

3348. glaber Lec. cygnæi Mann.

$3348 a$. americanus Mann. 3348b. Germari Mann.

\section{Brontes Fab.}

6) 3349. dubius Fab.

$3344 a$, truncatus Mots.

Y3350. debilis Lec.

\section{Hemipeplus Lat.}

3351. marginipennis Lec. 3352. microphthalmus $S z$.

Telephanus Er.

3353. velox Hald.

Psammœcus Lat.

33ə̃4. Desjardinsi Guér. muse Woll. Hubbardi Casey.

\section{CIR Y P'TOPIIA GID.T.}

\section{Telmatophilus Heer.}

3355. americanus Lec.

Loberus Lec.

3356. impressus Lec.
Tomarus Lec.

3357. pulchellus Lec.

3358. acutus Reit.

Antherophagus Lat.

3359. ochraceus Melsh. 
3360. convexulus Lec.

3361. suturalis $M i i k l$.

\section{Emphylus Fr.}

3362. americanus Lec.

\section{Henoticus Thom.}

Paramecosoma Curt.

3363. serratus Gyll.

\section{Cryptophagus Hbst.}

3364. cellaris Scop.

3365. 8-dentatus Mähl.

3366. 4-dentatus Mann.

3367. bidentatus $\mathbf{M} i k l$.

3368. difficilis Lec.

3369. debilis Lec.

3370. Lecontei G.\& $H$.

3371. saginatus Sturm.

3372. croceus Zimm.

3373. erinitus Zimm.

3374. fungicola $\mathrm{Zimm}$.

3375. nodangulus Zimm.
3376. tuberculosus Mäkl.

3377. 4-hamatus Makl.

3378. punctatissimus Maikl.

3379. ealifornicus Mann.

Cœnoscelis Thom.

3380. ferruginea Sahlb.

Atomaria Steph.

3381. vespertina $M \ddot{a} k l$.

3382. planulata $M a ̈ k l$.

3383. fuscicollis Mann.

3384. kamtschatica Mots.

33\$5. fulvipennis Mann.

3386. lepidula Mäkl.

3:37. lretula Lec.

33s. ochracea Zimm.

3.: 9 . ephippiata Zimm.

-._ ? mesomela Hbst.

Ephistemus Steph.

3390. apicalis Lec.

\section{MYCETOFIAGID $\mathrm{E}$.}

Mycetophagus Hellw.

3391. punctatús Say.

3392. californicus Horn.

3393. flexuosus Say.

3394. bipustulatus Melsh.

3395. pluriguttatus $L e c$.

3396. Melsheimeri Lec.

3396a. obscurus Lec.

3397. pluripunctatus Lec.

3398. pini Ziegl.

3399. tenuifasciatus Horn.

3400. obsoletus Melsh.

3401. confusus Horn.

\section{Triphyllus Lat.}

3402. humeralis Kirby.

3403. elongatus Lec.

\section{Litargus Er.}

3404. 6-punctatus Say.

3405. balteatus Lec.

3406. tetraspilotus Lec.
3407. didesmus Say.

3408. nebulosus Lec.

Typhœa Steph.

3409. fumata Linn.

\section{Berginus Er.}

3410. pumilus tec.

Myrmechixenis Chev.

Myrmecoxenus Chev.

3411. latridioides $\mathrm{Cr}$.

Hypocoprus Mots.

3412. formicetorum Mots.

Diplocœlus Guér.

3413. angusticollis Horn.

3414. brunneus Lec.

3415. rudis Lec.

—_ mus Reit. 
DERM ES'II D A.

Byturus Lat.

3416. unicolor $S(c y$.

3417. grisescens Lec.

Dermestes Linn.

3418. marmoratus Say.

3418a. Mannerheimii Lec.

3419. fasciatus $L e c$.

3420. caninus Germ.

murinus $\ddagger L e c$.

* nubilus Say. rattus $L e c$.

3421. talpinus Mann.

3422. sobrinus Lec.

3423. carnivorus Fab. mucoreus Lec.

3424. pulcher Lec. 3425. lardarius Linn. $3425 a$. signatus Lec. 3426. elongatus Lec. 3427. cadaverinus $F a b$. 3427a. domesticus Germ. 3428. vulpinus $F a b$. 3429. Frischii Kug.

Perimegatoma Horn.

3430. cylindricum Kirby.

3431. variegatum Horn.

3432. falsum Horn.

3433. Belfragei Lec.

Attagenus Lat.

'stit. piceus Oliv. dichrous Lec. megatoma Fab. rufipennis Lec.

3435. pellio Linn.

3436. Hornii Jayne.

3437. perplexus Jayne.

Dearthrus Lec.

3438. longulus Lec.
Acolpus Jayne.

3439. primus Jayne.

Trogoderma Lat.

3440. simplex Jayne.

3441. sternale Jayne.

3442. ornatum Say. pusillum Lec.

3443. tarsale Melsh. inclusum Lec. pallipes Ziegl.

Anthrenus Geoff.

3444. scrophulariæ Linn. flavipes Lec. thoracicus Melsh.

3445. varius $F a b$.

3446. musæorum Linn.

3447. claviger Er.

Cryptorhopalum Guér.

3448. balteatum Lec.

3449. hæmorrhoidale Lec.

3450. apicale Mann.

3451. ruficorne Lec.

3452. triste Lec.

Axinocerus Jayne.

3453. americanus Jayne.

Apsectus Lec.

3454. hispidus Melsh.

Orphilus Er.

3455. glabratus Fab. ater $\mathrm{Er}$.

subnitidus Lec.

\section{IIS'T ERI D $A$.}

Hololepta Payk.

3456. yúcateca Mars.

3457. cacti Lec.

3458. quadridentata $F a b$.
3459. vicina $L e c$.

3460. lucida Lec.

3461. fossularis Say.

3462. populnea Lec. 


\section{Hister Linn.}

3463. Klugii Mars.

3464. planipes Lee.

3465. perpunctatus Lec.

3466. subopacus Lec.

3467. guttifer Horn.

3468. arcuatus Say.

3469. sellatus Lec.

3470. Gloveri Horn.

3471. instratus Lec.

3472. biplagiatus Lec.

3473. Ulkei Horn.

3474. lucanus Horn.

3475. lævipes Germ.

3476. sexstriatus Lec.

3477. Harrisii Kirby.

3478. repletus Lec.

3479. merdarius Hoffm.

3480. interruptus Beauv.

3481. stygicus Lec.

3482. immunis Er.

3483. marginicollis Lec.

3484. cognatus Lec.

3485. remotus Lec.

34 f86. fodatus Lec.

3487. cœnosus Er.

3488. punctifer Payk.

3489. thoracicus Payk.

-3490. abbreviatus Fab.

3491. civilis Lec.

3492. semisculptus Lec.

3493. dispar Lec.

- 3494. depurator Say.

3495. furtivus $L e c$.

3496. incertus Mars.

3497. militaris Horn.

3498. curtatus Lec.

3499. Haldemanni Mars.

3500. defectus Lec.

3501. indistinctus Say.

3502. servus $E r$.

3503. bimaculatus Linn.

3504. tornatus Lec.

3505. sedecimstriatus Say.

3506. ambigena Lec.

3507. americanus Payk.

3508. perplexus Lec.

3509. exaratus Lec.

3510. nubilus Lec.
3511. pollutus Lec.

3512. relictus Mars.

3513. æneomicans Horn.

3514. venustus Lec.

3515. subrotundus Say.

3516. vernus Say.

3517. gentilis Horn.

3518. Saunieri Mars.

3519. carolinus Payk.

y 3520 . Lecontei Mars.

3521. aurelianus Horn.

† 3522. parallelus Say.

3523. æequus Lec.

3524. coarctatus Lec.

3525. punetiger Lec.

3526. basalis Lec.

3527. cylindricus Payk.

3528. attenuatus Lec.

3529. gracilis Lec.

Tribalister Horn.

3530. marginellus Lec.

Tribalus Er.

3531. americanus Lec.

3532. californicus Horn.

\section{Epierus Er.}

3533. regularis Beauv.

3534. novellus Zimm.

3535. pulicarius Er.

3536. ellipticus Lec.

3537. brunnipennis Mars.

3538. planulus Er.

3539. nasutus Horn.

Hetærius Er.

3540. morsus Irec.

3541. tristriatus Horn.

3542. brunnipennis Rand.

3543. Blanchardi Lec.

3544. californieus Horn.

ulkeus Horn.

3545. intricatus Horn.

Echinodes Zimm.

3546. setiger Lec.

3547. decipiens Horn. 
Onthophilus Leach.

3548. Lecontei Horn.

3549. alternatus Say.

3549a. nodatus Lec.

3549b. pluricostatus Lec.

\section{Peploglyptus Lec.}

3550. Belfragei Lec.

\section{Dendrophilus Leach.}

3551. punctulatus Say.

\section{Paromalus Er.}

3552. xqualis Say.

35.5. estriatus Lec.

3554. conjunctus Say.

3555. geminatus Lec.

3556. mimeticus Horn.

:35.57. 14-striatus Steph.

3558. lautus Zimm.

3559. opuntix Lec.

3560. tejonicus Horn.

35f1. consors Lec.

3562. gilensis Lec.

3563. tenellus $\mathrm{Er}$.

3564. bistriatus $E r$.

3565. difficilis Horn.

3566. debilis Lec.

3567. seminulum Er.

3568. teres Lec.

\section{Anapleus Horn.}

3569. marginatus Lec.

\section{Saprinus Er.}

3570. discoidalis Lec.

2571. rotundatus $\mathrm{Kug}$.

35.2. Behrensii Horn.

:573. planisternus Mars.

3ir.t. rugipennis Mars.

:5\%.). interstitialis Lec.

3576. pectoralis Lec.

357. obscurus Lec.

3.). . floridæ Horn.

3579. posthumus Mars.

35. 11 . pærninosus Lec.

:3) 1 . Copei Horn.

:Lis'. alienus Lec.
- 3583. lugens Er.

3584. imperfectus Lec.

/3585. pennsylvanicus Payk.

3586. oregonensis Lec.

:35sict. distinguendus Mars.

3587. impressus Lec.

:3588. infaustus Lee.

3589. æneicollis Mars.

/ 3590. assimilis Payk.

3591. cubæcola Mars.

3592. conformis Lec.

:3593. placidus $\mathrm{Er}$.

3594. veseus IFars.

3595. parumpunctatus Lec.

3596. convexiusculus Mars.

3597. minutus Lec.

3598. seissus Lec.

3599. scrupularis Lec.

3600 . wacoensis Horn.

3601. laridus Lec.

3602. insertus Lec.

:603. rquipunetatus Horn.

3604. ciliatus Lec.

3605. neglectus Mars.

3606. rubriculus Mars.

3607. vitiosus Lec.

3608. lubricus Lec.

3609. plenus Lec.

3610. fimbriatus Lec.

3610a. desertorum Mars.

3611b. orbienlatus Mars.

3611. acilinea Mars.

3612. ererulescens Lec.

3613. vestitus Lec.

3614. sphæroides Lec.

-3615. seminitens Lec.

3616. ferrugineus Mars.

:3617. fraternus Say.

- 3.11.. mancus Say.

3619. bigemmeus Lec.

3620. Fitchii Mars.

3621. estriatus Lec.

3622. dentipes Mars.

3623. braziliensis Payk.

3624. permixtus Lec.

3625. patruelis Lec.

3626. lucidulus Lec.

3627. æneipunctatus Horn.

3628. dimidiatipennis $L e c$.

:3629. gaudens Lec. 
3630. serrulatus Lec.

3631. sulcifrons Lec.

\section{Plegaderus Er.}

3632. Barbelini Mars.

3633. transversus $\boldsymbol{S} a y$.

3634. Sayi Mars.

3635. consors Horn.

3636. fraternus Horn.

3637. nitidus Horn.

\section{T'eretrius Er.}

3638. obliquulus Lec. 3639. placitus Horn.

3640 . americanus Lec.

3641. montanus Horn.

Teretriosoma Horn.

3642. chalybreum Horn.

\section{Abræus Leach.}

3643. Bolteri Lec.

\section{Bacanius Lec.}

3644. misellus Lec.

3645. tantillus Lec.

3646. punctiformis Lec.

Acritus Lec.

3647. maritimus Lec.

3648, arizonæ Horn.

3649. exiguus $E r$.

3650. floridæ Murs.

3651. discus Lec.

3652. fimetarius Lec.

3653. Sallei Horn.

3654. strigosus Lec.

3654a. conformis Lec.

3655. acaroides Mars.

3656. salinus Lec.

Aeletes Horn.

3657. politus Lec.

3658. brevisternus Mars.

3659. basalis Lec.

3660. simplex Lec.

\section{NI'T I D UIIID $A$.}

Brachypterus Er.

3661. urticæ $F a b$.

3662. troglodytes Murr.

3663. globularius Murr.

\section{Cercus Lat.}

3664. abdominalis $\mathrm{Er}$.

366.5. pennatus Murr.

3666. sericans Lec.

3667. bipustulatus Payk.

Amartus Lec.

3668. tinetus Mann. ferrugatus Murr.

3669. rufipes Lec.

\section{Anthonæus Horn.}

3670. agavensis $\mathrm{Cr}$.

Carpophilus Steph.

3671. уuсcæ $\mathrm{Cr}$.

3672. hemipterus Linn.
3673. pallipennis Say.

3674. dimidiatus Fab.

3675. melanopterus $\mathrm{Er}$.

3676. tempestivus Er.

3677. decipiens Horn.

3678. niger Say.

lugubris Murr.

3679. marginatus Er.

3680. corticinus Er.

3681. brachypterus Say.

3682. discoideus Lec.

3683. antiquus Melsh.

368t. zuni Casey.

Colastus Er.

3685. morio Er.

3686. maculatus $\mathrm{Er}$.

3687. semitectus Say.

3688. unicolor Say.

3689. truncatus Rand.

3689a. obliquus Lec.

3689b. limbatus Lec. 
Brachypeplus Er.

3690, glaber Lec.

Conotelus Er.

:691. stenoides Murr.

3692. obseurus Er.

3693. mexicanus MFur.

\section{Epuræa Er.}

3694. monogama $\mathrm{Cr}$.

3695. Hornii $\mathrm{Cr}$.

3696. helvola Er.

3697. rufa Say.

3698. integra Horn.

3699. ambigua Mann.

3700. Erichsonii Reit.

3701. rufida Melsh.

3702. papagona Casey.

3703. corticina Er.

3704. immunda Sturm.

3705. adumbrata Mann.

3706. avara Rand.

:307. fulvescens Horn.

3708. linearis Mi $i k l$.

3709. truncatella Mann.

3710. planulata Er.

3711. ovata Horn.

3712. peltoides Horn.

3713. restiva Linn.

3714. labilis $E r$.

3715. umbrosa Horn.

3716. obtusicollis Reit.

3717. scaphoides Horn.

3718. luteola Er.

- macrophthalma Reit.

__ placida Mräkl.

\section{Nitidula Fab.}

3719. bipustulata Linn.

3720. rufipes Linn.

3721. ziczac Say. bumeralis Lec. uniguttata Melsh.

\section{Stelidota Er.}

3722. geminata Say.

3723. 8-maculata Say.

3724. strigosa Gyll.
Prometopia Er.

3725. 6-maculata Say.

Phenolia Er.

$\checkmark 3726$. grossa Fab.

Omosita Er.

73727. colon Linn.

3728. discoidea Fab.

\section{Soronia Er.}

3729. guttulata Lec.

3730. undulata Say.

3731. Ulkei Lec.

Thalycra Er.

3732. concolor Lec.

Perthalycra Horn.

3733. Murrayi Horn.

Pocadius Er.

3734. helvolus Er.

3735. fulvipennis $\mathrm{Er}$. dorsalis Horn.

Orthopeplus Horn.

3736. quadricollis Horn.

Meligethes Steph.

3737. sævus Lee.

3738. brassicæ Scop. rufimanus $L \in c$.

3739. mutatus Har.

3740. seminulum Lec.

3741. pinguis Horn.

Oxycnemus Er.

Psilopyga Lec.

> 3742. histrina Lec.

3743. nigripennis Lec.

Amphicrossus Er.

3744. ciliatus Oliv.

3745. niger Horn. 


\section{Cyllodes Er.}

3746. biplagiatus Lec.

\section{Pallodes Er.}

3747. silaceus Er. pallidus Barud

\section{Cychramus Kug.}

3748. adustus Er.

3749. Zimmermanni Horn.

\section{Cybocephalus Er.}

3750. nigritulus Lec.

3751. californicus Horn.

_- unicolor Mots.

\section{Cryptarcha Shuck.}

3752. ampla Er.

3753. strigata Fab.

3754. concinna Mclsh.

Ips Fab.

3755. obtusus Say.

\& 3756. fasciatus Oliv. geminatus Melsh.
3757. sanguinolentus Oliv.

3758. confluentus Say.

3759. vittatus Say.

3760. eylindricus Lec.

Pityophaguis Shuck.

3761. cephalotes Lec.

3762. rufipennis Horn.

3763 . verticalis Horn.

\section{Smicrips Lec.}

Tisiphone Reit.

3764. hypocoproides Reit.

\section{Rhizophagus Hbst.}
3765. scalpturatus Mann.
\$766. cylindrieus Lec.
3767. dimidiatus Mann.
3768. brunneus Horn.
3769 . procerus Casey.
3770. bipunctatus Say.
3771. approximatus Lec.
:372. remotus Lec.
3773. minutus Mann.
- erythropterus MClsh.

\section{IU TRIDIID N.}

\section{Holoparamecus Curt.}

3774. pacificus Lec.

3775. singularis Beck.

Bonvouloiria Duval.

3776. parviceps Lec.

\section{Dasycerus Brong.}

3777. angulicollis Horn.

3778. earolinensis Horn.

\section{Stephustethus Lec.}

3779. liratus $L$ Jec.

\section{Latridius Hbst.}

3780. protensicollis Mann.

3781. minutus Linn.

3782. parallelocollis Mann.

3783. filiformis Gyll.

3784. cordicollis Mann.
3785. erenatus Lec.

3786. ruficollis Marsh.

37S7. cinnamopterus Mann.

3788 . strangulatus Mann.

3759. curtulus Tann.

3790. duplicatus LeC.

3791. laticollis Lec.

3792. maculatus Lee.

3793. opaculus Lec.

3794. tenuicornis Lec.

-_ aterrimus Mots.

- atripennis Mots.

- incisus Mots.

_... trilobatus Mots.

Coninomus Thom.

3795. fulvipennis Mann.

3796. carinatus Gyll.

Corticaria Marsh.

3797. grossa Lec. 


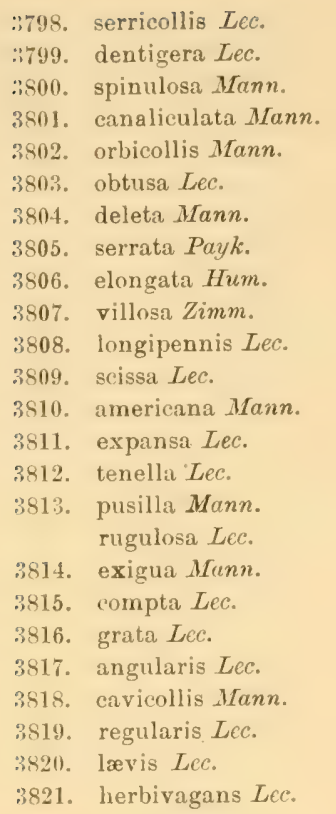

3822. morsa Lec.

3823. pumila Lec.

3824. subimpressa Zimm.

3825. rufula Lec.

3826. picta Lec.

3827. simplex Lec.

_ cylindrinotata Mots.

- debilis Mots.

- depressiuscula Mots.

_ ferruginosa Mots.

-_ helvola Mots.

__ inculta Mots.

- inermis Mots.

_.. nigripennis Mots.

_-. ovipennis Mots.

__ planiuseula Mots.

_. pullula Mots.

_- rectangula Mots.

_- salpingoides Mots.

___ sericella Mots.

- - striatopunetata Mots.

__ subangulata Mots.

Monœdus Lee.

3828. guttatus Lec.

\section{TIROCOSITID A.}

\section{Nemosoma Lat.}

3829. parallelum Melsh.

3830. cylindrieum Lec.

\section{Alindria Er.}

3831. cylindrica Serv.

3832. teres Melsh. æequalis Reit.

\section{Trogosita Oliv.}

38:3. virescens $F a b$. cyanea Reit. chloroida Mann. viridicyanea Mann.

3834. barbata Lec.

3835. yuceæ $\mathrm{Cr}$.

\section{Tenebrioides Pall.}

3836. mauritanica Linn. 3836a. nitida Horn. 3836b. crassicornis Horn.
3837. pleuralis Horn.

Q.3838. corticalis Melsh.

3838a. intermedia Horn.

3838b. dubia Melsh.

3838c. limbalis Melsh.

3839. nana Melsh.

3840. eollaris Sturm.

3841. sinuata Lec.

3841a. californica Horn.

3842. marginata Beauv.

$3542 a$. cucujiformis Horn.

3843. castanea Melsh.

3843a. laticollis Horn.

3843b. obscura Horn.

3843e. nigrita Horn.

3844. rugosipennis Horn.

3845. bimaculata Melsh.

3846. obtusa Horn.

3847. semicylindrica Horn.

_-_ depressior Beauv.

__ pusillima Mann.

___ subnigra Beauv. 
Peltis 111.

Ostoma Laich.

3848. Pippingskoeldi Mann.

3849. ferruginea Linn.

Calitys Thom.

3850. seabra Thunb.

\section{Grynocharis Thom.}

3851. 4-lineata Melsh.

3852. oregonensis $\mathrm{Cr}$.

3853. pilosula $\mathrm{Cr}$.

Dohrnii Reit.

lagrioides Reit.

Thymalus Duft.

3854. fulgidus $E r$.

Monotoma Hbst.

3855. producta Lec.

3856. picipes Hbst.

3857. fulvipes $\mathrm{Melsh}$.

3858. 4-foveolata Aubé.
3859. americana $A u b e ́$.

3860 . parallela Lec.

3861. mucida Lec.

3862. texana Horn.

3863. longicollis Gyll.

\section{Phyconomus Lec.}

3864. marinus Lec.

Hesperobænus Lec.

3865. abbreviatus Mots.

3866. rufipes Lec.

Europs Woll.

3867. pallipennis Lec.

3868. longicollis Horn.

\section{Bactridium Isec.}

3869. ephippigerum Guér.

3870, striatum Lec.

3871. striolatum Reit. corpulentum Reit.

3872. cavicolle Horn.

\section{DEIRODONTIID A.}

Derodontus Lec.

3873. maculatus Melsh.

3874. trisignatus Mann.
Peltastica Mann.

3875. tuberculata Mann.

\section{BYIRIHID $\boldsymbol{A}$.}

Nosodendron Lat.

3876. unicolor Say.

3877. californieum Horn.

\section{Amphicyrta Er.}

3878. chrysomelina $\mathrm{Er}$.

3879. dentipes $E r$.

3880. simplicipes Mann.

Simplocaria Steph.

3881. metallica Sturm.

3882. nitida Mot.s.

\section{Pedilophorus Steff:}

y 3883. oblongus Lec.

3884. acuminatus Mann.
3885. æneolus Lec.

3886. subcanus Lec.

Cytilus Er.

$\checkmark$ 3887. sericeus Forst.

3888. trivittatus Melsh.

Byrrhus Linn.

3889. americanus Lec.

3890. cyclophorus Kirby. concolor Kirby.

3891. geminatus Lec.

3892. Pettitii Horn.

3893. Kirbyi Lec.

3894. eximius Lec.

3895. murinus $F a b$. 
Syncalypta Steph.

3896. strigosa Melsh.

3897. grisea Lec.

3898. echinata Lec.

3899. setulosa Mann.

3900. albonotata Lec.

\section{Limnichus Lat.}

3901. punctatus Lec.

3902. nebulosus Lec.

3903. analis Lec.

3904. ater Lec.
3905. montanus Lec.

3906. californicus Lec.

3907. nitidulus Lec.

3908. lutrochinus Lec.

3909. ovatus Lec.

\section{Bothriophorus Muls.}

Physemus Lec.

3910. minutus Lec.

Chelonarium Fab.

3911. Lecontei Thom.

\section{GEORYSAIDA.}

Georyssus Lat.

3913. californicus Lec.

3912. pusillus Lec.

\section{PAIR NIDN.}

Psephenus Hald.

3914. Lecontei Lec.

3915. Haldemanni Horn.

\section{Lara Lec.}

3916. avara Lee.

Lutrochus Er.

3917. lutens Lcc.

Throscinus Lee.

8918. Crntchii Lec.

\section{Pelonomus Er.}

3919. obscurus Lcc.

\section{Dryops Oliv.}

Helichus Er.

3920. productus Lec.

3921. lithophilus Germ.

3922. fastigiatus Say.

3923. striatus Lec.

3924. suturalis Lec.

Elmis Lat.

.3925, vittatus Melsh.

3926. concolor Lec.

:927. bivittatus Lec.
3928. 4-notatus Say.

3929. elegans $L e c$.

3930. fastiditus Lec.

3931. ovalis Lec.

3932. corpulentus Lec.

3933. divergens Lec.

3934. 4-maculatus Horn.

3935. seriatus Lec.

3936. glaber Horn. vulneratus Lec.

:937. nitidulus Lee.

3938. latiusculus Lec.

3939. mostus Horn. angustus Lec.

3940. abnormis ITorn.

3941. pusillus Lec.

3942. foveatus Lec.

3943. similis Horn.

3944. ferrugineus Horn.

3945. cresus Lec.

\section{Stenelmis Dup.}

3946. linearis Zimm.

3947. sinuatus Lec.

3948. crenatus Say.

3949. bicarinatus Lec.

3950 . 4-maculatus Horn.

3951. vittipennis Zimm.

___ elongatus Mots. 
-_ ? humerosus Mots. ? sordidus Mots.

Macronychus Müll.

3952. glabratus Say.
3953. parvulus Horn.

Ancyronyx Er.

3954. variegatus Germ.

\section{HET EIROCERID}

Heterocerus Fab.

3955. gnatho Lec.

3956. labratus Lec.

3957. ventralis Melsh.

3958. auromicans Kies.

3959. cuniculus Kies.

3960. tristis Mann.

3961. fatuns Kies.

3962. substriatus Kies.
3963. miser Kies.

3964. mollinus Kies.

3965. collaris Kies.

3966. limbatus Kies.

3967. luteolus Lec.

3968. pallidus Say.

3969. pusillus Say.

3970. undatus Melsh.

__ brunneus Melsh.

\section{DASCYYLI D A.}

Macropogon Mots.

3971. testaceipennis Mots.

3972. piceus Lec.

3973. rufipes Horn.

\section{Eurypogon Mots.}

3974. niger Melsh.

3975. ealifornicus Horn.

\section{Allopogon Horn.}

3976. villosus Horn.

\section{Stenocolus Lec.}

3977. scutellaris Lec.

\section{Dascyllus Lat.}

y 3978. Davidsonii Lec. 3979. plumbeus Horn.

Anorus Lec.

3980. piceus Lec.

Odontonyx Guér. 3981. trivittis Germ.

Anchytarsus Guér. 3982. bicolor Melsh.
Anchycteis Horn.

3983. velutina Horn.

Aræopus Lec.

3984. monachus Lec.

Brachypsectra Lec.

3985. fulva Lec.

Ptilodactyla Lat.

3986. serricollis Say. 3987. angustata Horn.

Eucinetus Germ.

3988. infumatus Lec.

3989. oviformis Lec.

3990. testaceus Lec.

3991. punctulatus Lec.

3992. morio Lec.

3993. terminalis Lec.

3994. strigosus Lec.

\section{Ectopria Lec.}

3995. nervosa Mlelsh.

Dicranopselaphus Guér.

3996. variegatus Horn. 
Acneus Horn.

3997. quadrimaculatus Horn.

Prionocyphon Redt.

3998. discoideus Say.

3999. limbatus Lec.

Microcara Thom

4000. explanata Lec.

Helodes Lat.

4001. apicalis Lec.

4002. maculicollis Horn.

4003. pulchella Guér.

4004. fuscipennis Guér.

4005. thoracica Guér.

Scirtes III.

4006. orbiculatus $F a b$.
4007. tibialis Guér.

4008. Troberti Guér.

- californicus Mots.

Cyphon Payk.

4009. robustus Lec.

4010. ruficollis Say.

4011. brevicollis Lec.

4012. exiguus Horn.

4013. concinnus Lec.

4014. obscurus Guér.

4015. collaris Guér.

4016. variabilis Thunb.

4017. padi Linn.

4018. impressus Lec.

Placonycha Horn.

4019. Edwardsii Lec.

\section{RH IPICEIR I D A.}

Zenoa Say.

4020. picea Beauv.

Sandalus Knoch.

- 4021. petrophya Knoch.
4022. porosus Lec.

4023. niger Knoch. scabricollis Hald.

4024. californicus Lec.

\section{EIA'TERID.E.}

Melasis Oliv.

4025. pectinicornis MFelsh.

Tharops Lap.

4026. nubilus Bonv.

4027. ruficornis Say. obliquus Say.

Dendrocharis Guér.

4028. flavicornis Guér.

Stethon Lec.

4029. pectorosus Lec.

Deltometopus Bonv.

4030. amonicornis Say. ereptus Bonv.

? monilicornis Mrann.
Dromæolus Kies.

4031. basalis Lec.

4032. californicus Bonv.

4033. cylindricollis Say.

4034. sejunctus Bonv.

4035. salsus Bonv.

4036. Marsenli Bonv.

4037. novitius Bonv.

4038. striatus Lec.

Phænocerus Bonv.

4039. americanus Horn.

Fornax Lap.

40.40. badius Melsh.

4041. calceatus Say.

4042. Hornii Borv.

4043. molestus Bonv.

404. orchesides Newm. 
4045. bicolor Melsh.

4046. ? rufipes Melsh.

\section{Adelothyreus Bonv.}

4047. Dejeani Bonv.

Entomophthalmus Bonv.

4048. rufiolus Lec.

\section{Microrrhagus Esch.}

4049. humeralis Say.

4050. subsinuatus Lec.

4051. imperfectus Lec.

4052. impressicollis Bonv. mucidus Bonv.

4053. oblitus Bonv.

4054. pectinatus Lec.

4055. triangularis Say.

\section{Hypoccius Esch.}

4056. frontosus Say.

4057. terminalis Lec.

\section{Nematodes Lat.}

4058. atropos Say.

4059. penetrans Lec.

4060. punctatus Lec.

\section{Schizophilus Bonv.}

4061. subrufus Rand.

\section{Cryptostoma Lat.}

4062. Dohrnii Horn.

Phlegon Lap.

4063. heterocerus Say.

Anelastes Kirby.

4064. Drurii Kirby. 4064a. Latreillei Lec.

Epiphanis Esch.

4065. cornutus Esch.

\section{Xylobius Lat.}

4066. cylindriformis Horn.
Hylochares Lat.

4067. nigricornis Say.

Sarpedon Bonv.

4068. scabrosus Bonv.

Agrypnus Esch.

4069. Sallei Lec.

4070. Schottii Lec.

\section{Adelocera Lat.}

4071. impressicollis Say.

4072. marmorata Fab.

4073. sparsa Cand.

4074. pyrsolepis Lec.

4075. modesta Boisd.

4076. discoidea Web.

4077. rorulenta Lec.

4078. aurorata Lec.

4079. maculata Lec.

4080. avita Say.

4081. obtecta $S \alpha y$.

4082. brevicornis Lec.

4083. profusa Cand.

\section{Lacon Lap.}

4084. curtus Lec.

4085. rectangularis Say.

Meristhus Cand.

4086. cristatus Horn.

4087. scobinula Cand.

\section{Chalcolepidius Esch.}

4088. rubripennis Lec.

y 4089. Webbii Lec.

4090. smaragdinus Lec.

4091. viridipilis Say.

Alaus Esch.

4092. Iusciosus Hope.

$>$ 4093. oculatus Linn.

y 4094. nuyops Fab.

4095. melanops Lec.

\section{Hemirhipus Lat.}

4096. fascicularis $F a b$. 


\section{Cardiophorus Esch.}

4097. convexus Say.

4098. floridæ Cand.

4099. erythropus Er.

4100. insulsus Cand.

4101. Edwardsii Horn.

4102. togatus Horn.

4103. renells Horn.

4104. eardisce Say.

4104a. Dejeanii Lec.

4105. fenestratus Lec.

4106. stigmaticus Cand.

4107. tumidicollis Lec.

4108. cunvexulus Lec.

4109. gagates $\mathrm{Er}$.

4116. longior Lec.

411. luridipes Cand.

4112. fulvipes Lec.

4113. tenebrosus Lec.

4114. obscurus Lec.

4115. amplicollis Mots.

4116. latiusculus Esch.

4117. lrevicollis $\mathrm{Er}$.

4118. robustus $L e c$.

- ealiforniens Mann.

- filius Rand.

\section{Coptostethus Woll.}

4119. americanus Horn.

\section{Horistonotus Cand.}

4120. vulneratus Horn.

4121. pullatus Horn.

4122. densus Lec.

4123. inanus Lec.

4124. basalis Horn.

4125. transfugus Lec.

4126. definitus Horn.

4127. sufllatus $L_{c c}$.

4128. simplex Lec.

4129. curiatus Say.

4131). gracilis Horn.

4131. exoletus $\mathrm{Er}$.

4132. Uhlerii Horn.

4133. mitis Horn.

\section{Esthesopus Esch.}

4134. claricollis Say.

4135. præditus Horn.
4136. pareus Horn.

hepaticus $\ddagger$ Auct.

4137. dispersus Horn. humilis $\ddagger$ Auct.

4138. pusio Horn.

4139. bicolor Horn.

\section{Aptopus Esch.}

4140. peregrinus Horn.

\section{Cryptohypnus Esch.}

4141. squalidus Lec.

4142. planatus Lec.

4143. funebris Cand.

414. hyperboreus Gyll.

4145. Sanborni Horn.

4146. grandicollis Lec.

4147. littoralis Esch.

1148. impressicollis Munn.

4149. abbreviatus Say.

4150. bicolor Esch.

4151. restrictus Mann.

4152. musculus Esch.

4153. tumescens Lec.

4154. striatulus $L e c$.

4155. dubius Horn.

4156. guttatulus Melsh.

4157. choris Say.

4158. pulchellus Linn.

4159. ornatus Lec.

4160. gentilis Lec.

1161. pectoralis Say.

416i2. obliquatulus Melsh.

4163. futilis $L e c$.

4164. rstivus Horn.

4165. perplexus Horn.

Oedostethus Lee.

4166. femoralis Lec.

Anchastus Lec.

4167. cinereipennis Mann. puberulus Mann.

4168. tantillus Mann.

4169. regularis Mots.

4170. hicarinatus Lec.

4171. digitatus Lec.

4172. rufus Cand.

4173. signaticollis Germ. 
4174. binus Say.

4175. frontalis Horn.

4176. militaris Cand.

4177. sericeus Horn.

4178. bicolor Lec.

4179. asper Lec.

\section{Monocepidius Esch.}

4180. xysticus Cand.

4181. lividus $D e G$.

4182. aversus Lec.

4183. suturalis Lec.

4184. lepidus Lec.

4185. vespertinus $F a b$. texanus Cand.

4186. debilis Lec.

4187. athoides Lec. mutuus Horn.

4188. sordidus Lec.

4189. robustus Horn.

4190. auritus Hbst.

finitimus Casey.

4191. bellus Say.

4192. blandulus Lec.

\section{Dicrepidius Esch.}

4193. ramienrnis Beauv.

4194. corvinus Cand.

4195. palmatus Cand.

Ischiodontus Cand.

Tricrepidius Mots.

4196. ferreus Lec. triangulicollis Mots.

4197. soleatus Say. oblitus Cand.

4198. fuscus Lec.

Blauta Lec.

4199. eribraria Germ.

\section{Elater Linn.}

4200. hepaticus Melsk.

4201. insignis Lec.

4202. rhodopus Lec.

4203. fastus Lec.

4204. rubriventris $L e c$.

1205. partitus Cand.
4206. melinus Lec.

4207. Sturmii Germ.

4208. Phelpsii Horn.

4209. Horni Cand.

4210. cordatus Horn.

4211. Behrensi Horn.

4212. cordifer Lec.

4213. ater Lec.

4214. manipularis Cand.

4215. longicornis Lec.

4216. carbonicolor Esch.

4217. pedalis Germ. deletus Lec.

4218. nigrinus Payk. anthracinus Lec.

4219. mixtus Hbst.

fusculus $L e c$.

Iacustris Lec.

miniipennis Lec.

4220. pullus Germ.

4221. nigricollis Hbst.

4222. linteus Say.

4223. discoidens $F a b$.

4224. vitiosus $L e c$.

4225. laesus $L e c$.

4226. Sayi Lec.

4227. impolitus Melsh.

-4228. socer trec.

< 4229. rubricollis Hbst.

1230. semicinetus Rand.

protervus Lec.

1231. militaris Harr.

42:32. subtilis Lec.

423\%. mørens Lec.

4234. luctuosus Lec.

71235 . nigricans Germ.

molestus Lec.

4236. rubricus Say.

42:7. lateralis Lec.

12:38. atripennis Horn. violaceipennis Cand.

12::9. collaris Say. palans Lec.

4240. torquatus Lec.

1241. nevadensis Lec.

4242. sanguinipennis Suy.

4243. xanthomus Germ.

1244. dimidiatus Lec.

Lecontei Cand. 
4245. apicatus Say. phoenicopterus Germ.

4246. affinis Lec.

4247. obliquus Say. areolatus Say.

4248. pusio Germ.

_._ ? ignobilis Boh.

- ? fuscatus Melsh.

- ? ?emivitatus say. $=44 \sqrt{3}$

__ ? basalis Rand.

_- ? macilentus Rand.

\section{Elatrinus Horn.}

4249. anthrax Horn.

\section{Drasterius Esch.}

4250. grandicollis Horn.

4251. asper Lec.

4252. livens Lec.

4253. elegans $F a b$.

dorsalis Say. marginicollis Horn.

4254. amabilis Lec. fretus Casey.

4255. cribratus Lec.

4256. simiolus Cand.

4257. debilis Lec.

\section{Megapenthes Kies.}

4258. tartareus Lec.

4259. turbulentus $L c c$.

4260. aterrimus Horn.

4261. granulosus Melsh.

4262. limbalis Hbst.

4263. nigriventris Lec.

4264. rufilabris Germ.

4265. elegans Horn.

4266. stigmosus Lec.

4267. 4-maculatus Horn.

4268. Rogersii Horn.

4269. lepidus Lec.

4270. angularis Lec.

\section{Ludius Lat.}

Crigmus Lec.

4271. attenuatus Say.

4272. Lecontei Horn.

4273. ater Cand.

4274 pinguis Horn.
7 4275. abruptus Say.

4276. texanus Lec.

4277. hepaticus Germ.

Orthostethus Lac.

4278. infuscatus Germ.

Agriotes Esch.

4279. maneus Say.

4280. stabilis Lec.

4281. insanus Ceand.

4282. fucosus $L e c$.

4283. ferrugineipennis Lec.

4284. imperfectus Lec.

4285. sparsus Lec.

4286. pubescens Melsh.

4287. limosus Lee.

4288. hispidus Lec.

4289. opaculus Lec.

4290. oblongicollis Melsh.

4291. nevadensis Lec.

4292. apicalis Lec.

4293. avulsus Lec.

4294. Thevenetii Horn.

4295. torquatus Lec.

4296. montanus Lec.

\section{Dolopius Esch.}

4297. lateralis Esch. macer Lec. simplex Mots.

Betarmon Kies.

4298. bigeminatus Rand.

\section{Glyphonyx Cand.}

4299. recticollis Say.

4300. testaceus Melsh.

4301. quietus Say.

4302. inquinatus Say.

4303. mimeticus Horn.

Melanotus Esch.

4304. corticinus Say.

4305. longulus Lec.

4306. macer $L \in c$.

4307. decumanus Er.

4308. canadensis Cand. 
4309. despectus Cand.

4310. clandestinus Er.

4311. secretus Lec.

4312. ignobilis Melsh.

4313. depressus Melsh.

4314. angustatus $\mathrm{Er}$.

4315. trapezoideus Lec.

4316. tanicollis Lec.

4317. Leonardi Lec.

4318. scrobicollis Lec.

4319. texanus Cand.

4320. castanipes Payk.

4321. glandicolor Melsh.

4322. fissilis Say.

4323. communis Gyll.

4324. exuberans Lec.

4325. parumpunctatus Melsh.

4326. effetus Cand.

4327. verberans Lec.

1328. emissus Lec.

4329. infaustus Lec.

4330. cribulosus Lec.

4331. cribricollis Canr.

4332. paganus Cand.

4333. pertinax Say.

4334. dubius Lec.

4335. tenàx Say.

4336. americanus Hb.st.

4337. insipiens Say.

4338. gradatus Lec.

4339. opacicollis Lec.

4340. variolatis Lec.

4341. oregonensis Lec.

4342. morosus Cand.

4343. sagittarius Lec.

4344. paradoxus Melsh.

4345. abdominalis $E r$.

4346. vetulus $E r$.

4347. similis Kirby.

\section{Limonius Esch.}

1348. auripilis $S \alpha y$.

4349. fulvipes Cand.

4350. mirus Lec.

4351. Crotchii Horn.

4352. discoideus Lec.

4353. aurifer Lec.

4354. stigma Hbst.

4355. griseus Beauv.

4356. interstitialis Melsh.
7357. confusus Lec.

7 4358. plebejus Say.

4359. infuscatus Mots.

4360. oceidentalis Cand.

4361. æger Lec.

4362. pectoralis Lec.

1303. quercinus Say.

4364. maculicollis Mots.

4365. ornatulus Lec.

humeralis Cand.

4366. basillaris Say. semiæneus Lec.

4367. consimilis Walk.

4368. nitidulus Horn.

4369. subauratus Lec.

4370. pilosus Lec.

4371. californicus Mann.

4372. canus Lec.

4373. propexus Cand.

4374. anceps Lec.

4375. agonus Say.

4376. ornatipennis Lec.

4377. definitus Ziegl.

4378. nimbatus Say.

-_ ? angulatus Mots.

- ? Havipes Fab.

-. ? subcostatus Mots.

- ? elypeatus Mots.

__ ? mandibularis Mots.

\section{Campylus Fisch.}

4379. productus Rand.

4380. denticornis Kirby.

4381. variabilis Esch.

_- ? fulvus Mots.

\section{Pityobius Lec.}

4.ix2. anguinus Lec.

4.ง3. Murrayi Lec.

Athous Esch.

4384. Brightwelli Kirby.

4385. acanthus Say.

4386. opilinus Cand.

4387. undulatus Host.

4388. axillaris Horn.

4389. excavatus Mots.

4390. cucullatus Say.

4391. ferruginosus Esch. 
4392. simplex Lec.

4393. fossularis Lec.

4394. nigripilis Mots.

4395. seapularis Say.

4396. equestris Lec.

4397. rufiventris Esch.

4398. posticus Melsh.

4399. rufifrons Rand.

4400. eribratus Lec.

4401. limbatus Lec.

4402. seissus Lec.

4403. vittiger Lec.

4404. triundulatus Mann.

4405. orophilus Har.

4406. affinis Coup.

4407. pallidipennis Mann.

\section{Leptoschema Horn.}

4408. protractum Horn.

4409. bicolor Lec.

4410. discalceatum Suy.

\section{Bladus Lec.}

4111. quadricollis Say.

\section{Oestodes Lec.}

4412. tenuicollis Rand.

413. puncticollis Horn.

\section{Paranomus Kies.}

4414. costalis Payk.

4115. estriatus Lec.

4416. pictus Cand.

4417. decoratus Mann.

\section{Nothodes Lec.}

4118. dubitans Lec.

\section{Sericosomus Steph.}

4419. honestus Rand. fusiformis Lec.

4420. incongruus Lec.

4421. viridanus Say.

4422. silaceus Say.

4423. debilis Lec.

4424. Havipennis Mots.

4425. humeralis Mots.

\section{Corymbites Lat.}

4426. virens $S c h r$.

4427. vernalis Hentz.

i 442s. tesselatus Linn.

4429. cribrosus Lee.

4431. maurus Lec.

4431. anthrax Lee.

4432. obscurus Lec.

4433. resplendens Esch.

4434. cylindriformis Hbst.

4435. atropurpureus Melsh.

4436. fusculus Lec.

4437. divaricatus Lec.

4438. limoniiformis Horn.

4439. fulvipes Bland.

4440. protractus Lec.

4441. furtivus Lec.

4442. jaculus Lee.

4443. pyrrhos Hbst.

4444. bivittatus Melsh.

4445. longicornis Horn.

4446. volitans Esch.

4447. vulneratus Lec.

4448. signaticollis Melsh.

4449. spinosus Lee.

4450. tarsalis Melsh.

4451. caricinus Germ.

4452. monticola Horn.

4453. xanthomus Horn.

4454. mœrens Lec.

455. angularis Lec.

4456. mendax Lec.

4457. insidiosus Lee.

4458. falsificus Lec.

4459. Copei Horn.

4460. teres Lec.

4461. umbripennis Lec.

4462. opaculus Lec.

4463. appressus Rund.

4464. trapezium Lec.

4465. rupestris Germ.

4466. sulcicollis $S a y$.

4467. morulus Lcc.

4468. æthiops Hbst.

4469. colossus Lec.

4471. angusticollis Mann.

4471. fraternus Lec.

472. ochreipennis Lec.

473. trivittatus Lec.

4474. sagitticollis Esch. 
4475. fallax Say.

4476. medianus Germ.

4477. planulus Lec.

4478. triundulatus Rand.

4479. hamatus Say.

4480. propola Lec.

4481. nigricollis Bland.

4482. hieroglyphicus Say.

4483. Semivittatus Say.

4484. cruciatus Linn.

$4484 a$. Edwardsii Horn.

4485. Suckleyi Lec.

4486. æripennis Kirby.

4487. splendens Ziegl.

4488. carbo Lec.

4489. Breweri Horn.

4490. conjungens Lec.

4491. pruininus Horn.

4492. obversus Horn.

4493. leucaspis Germ.

4494. aratus Lec.

4495. metallicus Payk.

4496. inflatus Say.

4497. crassus Lec.

4498. rotundicollis Say.

_- ? spectabilis Mann.

- ? serricornis Mann.

_- ? undulatus Rand.

-- ? oblongoguttatus Mots.

- ? decoratus Mann.

-- ? parvicollis Mann.

__ ? rufipes Mots.

Oxygonus Lec.

4499. obesus Say.

4500. ater Horn.

Asaphes Kirby.

4501. hirtus Cand.

4502. curbonatus Lec.

4503. morio Lec.

4504. dilaticollis Mots.

4505. tumescens Lec.

4506. oregonus Lec.

4507. soccifer Lec.

4508. indistinctus Lec.

4509. decoloratus Say.

4510. memnonius Hbst.

4511. bilobatus Say.
Pyrophorus III.

4512. physoderus Germ.

Melanactes Lec.

4513. procerus Lec.

4514. piceus $D e G$.

4515. densus Lec.

4516. morio $F a b$.

4517. puncticollis Lec.

4518. consors Lec.

4519. Reichei Germ.

\section{Eniconyx Horn.}

4520. pullatus Horn.

4521. gracilis Horn.

\section{Aphricus Lec.}

4522. californicus $L e c$.

\section{Aplastus Lec.}

Anamesus Lec.

4523. angusticollis Horn.

4524. tenuiformis Horn.

4525. corymbitoides Horn.

4526. speratus Lec.

4527. optatus Lec.

convexicollis $q$ Lec.

4528. molestus Horn.

\section{Plastocerus Lec.}

4529. Schaumii Lec.

4529a. frater Lec.

4529b. macer Horn.

Euthysanius Lec.

4530. lautus Lec.

4531. pretiosus Lec..

Cebrio Oliv.

Anachilus Lec.

4532. bicolor Fab.

confusus Lec.

simplex Lec.

4533. mandibularis Lec.

4534. estriatus Horn. 


\section{Scaptolenus Lec.}

4535. Lecontei Sallé.

4536. nereatus Horn.

4537. estriatus Lee.

\section{Perothops Er.}

4538. rnucida Gyll.
4539. Witticki Lec.

- cervina Lac.

\section{Cerophytum Lat.}

4540. convexicolle Lec.

4541. pulsator Hald.

\section{TH ROSCII A.}

Drapetes Redt.

4542. geminatus Say.

4543. 4-pustulatus Bonv.

4544. nitidus Melsh.

4545. rubricollis Lec.

\section{Throscus Lat.}

4546. validus Lec.

4547. punctatus Bonv.

4548. invisus Horn.

4549. constrictor Say. calocerus Bonv.
4550. alienus Bonv.

4551. convergens Horn.

4552. pugnax Horn.

4553. Chevrolati Bonv.

4554. mendax Horn.

4555. sejunctus Horn.

4556. parvulus Lec.

4557. sericeus Lec.

4558. debilis Horn.

\section{Pactopus Lec.}

4559. Hornii Lec.

\section{$\checkmark$ IBUPRESTID $A$.}

Gyascutus Lec.

- 4560. planicosta Lec.

- 4561. obliteratus Lec.

4562. carolinensis Horn.

. 4563. cuneatus Horn.

Hippomelas Lap. \& Gory.

- 4564. spheriicus Lec.

.4565 . celatus Lec.

4566. californieus Horn.

Halecia Lap. \& Gory.

4567. gentilis Horn.

\section{Chalcophora Sol.}

4568. angulicollis Lec.

4569. virginiensis Drury.

$4569 c$. lacustris $L c c$.

C. 4570 , liberta Germ.

. 4571. georgiana Lec.

C. 4572. fortis $L c c$.

C. 4573. campestris Say.

4574. Fulleri Horn.
Psiloptera Sol.

4575. Drummondi Lap. \& Gory.

- 4575r. Webbii Lec.

Dicerca Esch.

4576. prolongata Lec.

7 4577. divaricata Say.

C 4578. pugionata Germ.

4579. obscura Fab.

Ch 4579a. lurida Fab.

4580. lepida $L c c$.

4581. spreta Gory.

Ch 4582. asperata Lap. \& Gory.

4583. tenebrosa Kirby.

4583a. chrysea Melsh.

4584. californica $\mathrm{Cr}_{r}$.

4585. tuberculata Chev.

4586. lugubris Lec.

4587. sexualis $\mathrm{Cr}$.

4588. punctulata Sch.

4589. pectorosa tec.

4590. Hornii $\mathrm{Cr}$.

4591. mutica Lcc. 
Trachykele Mars.

4592. Blondeli Mars.

4593. Lecontei Gory.

Poecilonota Esch.

Ch 4594. cyanipes Say.

4595. ferrea Melsh.

Ch-4596. thureura $\$ a y$.

4597. debilis Lec.

\section{Buprestis Linn.}

4598. rufipes Oliv.

4599. Gibbsii Lec.

4600. confluens Say.

$\checkmark$ 4601. lineata $F a b$.

4602. consularis Gory.

4603. connexa Horn.

4604. Nuttalli Kirby.

4605. læviventris Lec.

>े 4606. maculiventris Say.

$4606 a$. subornata Lec.

4606b. rusticorum Kirby.

C 4607. fasciata $F a b$.

4607a. Langii Mann.

4608. sulcicollis Lec.

4609. striata $F a b$.

4610. tauta Lee. aulenta $\operatorname{Sin} n)$

4611. adjecta Lec.

7612. decora Fab.

? viridula Oliv.

4613. ultramarina Say.

4614, apricans Hbst.

$4610 \frac{1}{2}$ Villosa fic

Cinyra Lap. \& Gory.

4615. gracilipes Melsh.

\section{Melanophila Esch.}

4616. miranda Lec.

4617. notata Lap. \& Gory.

4617a. opaca Lec.

4618. consputa Lec.

4619. longipes Say.

4620. atropurpurea Say.

4621. Drummondi Kirby.

4622. fulvoguttata $H a r r$.

4623. gentilis Lec.

4624. intrusa Horn.

4625. æneola Melsh.

4626. obtusa Horn.
Anthaxia Esch.

4627. salicis $F a b$.

4628. eneogaster Lap.

imperfecta Lec.

inornata Rund.

strigata Lec.

nanula Casey.

simiola Cusey.

renescens Casey.

4629. deleta Lec.

4630. viridifrons Lap.

4631. viridicornis Say.

4632. cyanella Gory.

4633. quercata Fab.

4634. Havimana Gory.

-__ ? bivittata Gory.

\section{Xenorhipis Lec.}

4635. Brendeli Lec.

\section{Chrysobothris Esch.}

4636. octocola Lec.

4637. atabalipa Lap.

4638. exesa Lec.

4639. femorata $F a b$.

4639a. alabamæ Gory.

4639b. 4-impressa Lap. \& Gory.

4639c. Lesueuri Lap. \& Gory.

4639d. fastidiosa Gory.

4639e. obscura Lec.

4639f. semisculpta Lec.

4640. Horicola Gory.

4641. cuprascens Lec.

4642. contigua Lec.

4643. ignicollis Horn.

4644. 4-lineata Lec.

4645. texana Lec.

4646. carinipennis Lec.

4617. dentipes Germ.

4648. californica Lec.

4649. vuleanica Lec.

4650. trinervia Kirby.

4651. scabripennis Lap. \& Gory.

4652. pusilla Lap. d: Gory.

4653. debilis Lec.

$4653 \alpha$. disjuncta Lec.

4654. deleta Lcc.

4655. acuminata Lec.

4656. gemmata Lec. 
4657. 6-signata Say.

$4657 \alpha$. analis Lec.

4658. chrysoela Ill.

4659. chlorocephala Gory.

4660. azurea Lec.

4661. Harrisii Hentz.

4662. æneola Lec.

4663. Ulkei Lec.

4664. atrofasciata Lec.

- cribraria Mann. errans Gory. dissimilis Gory. posticalis Lap. \& Gory. subcylindrica Mots.

_- ? frontalis Oliv.

- ? thoracica Fab.

Actenodes Lac.

4665. auronotata Lap. \& Gory.

4666. acornis Say.

4667. calcarata Chev.

\section{Belionota Esch.}

__ californica Mots.

\section{Dystaxia Lec.}

4668. Murrayi Lec. ? Lecontei Thom.

\section{Schizopus Lec.}

4669. lætus Lec.

4670. Sallei Horn.

\section{Thrincopyge Lec.}

4671. alacris $L e c$.

4672. ambiens Lec.

4673. lætifica Horn.

\section{Polycesta Sol.}

4674. californica Lec.

4674a. elata Lec.

4675. velasco Lap. \& Gory.

Acmæodera Esch.

4676. cuprina Spin.

4677. amplicollis Lec.

4678. opacula Lec.
4679. amabilis Horn.

4680. flavomarginata Gray.

4681. macra Horn.

4682. robusta Horn.

4683. pubiventris Horn.

4684. lanata Horn.

1685. plagiaticauda Horn.

4686. miliaris Horn.

4687. sparsa Horn.

4688. tuta Horn.

4689. Hepburnii Lec.

4690. connexa Lec.

4691. acuta Lec.

4692. ornata Fab.

4693. flavosticta Horn.

4694. Dohrnii Horn.

4695. decipiens Lec.

4696. stellaris Chev.

4697. variegata Lec.

4698. mima Gory.

4699. pulchella Host. mixta Lec.

4700. obtusa Horn.

4701. consors Horn.

4702. subbalteata Lec.

4703. texana Lec.

1704. versuta Horn.

4705. guttifera Lec.

4706. mariposa Horn.

4707. culta Web.

4708. 4-vittata Horn.

4709. gemina Horn.

4710. gibbula Lec.

4711. comata Lec.

4712. alacris Horn.

\section{Ptosima Sol.}

4713. gibbicollis Say.

4714. Walshii Lec.

Tyndaris Thom.

4715. cincta Horn.

Chrysophana Lec.

4716. placida Lec.

Mastogenius Sol.

4717. subcyaneus Lec. 
Eupristocerus Deyr.

\section{$>$ 4718. cogitans $W e b$.}

\section{Agrilus Steph.}

4719. fuscipennis Gory.

4721). arcuatus Say.

Y 4721. ruficollis Fab.

4722. torquatus Lec.

4723. fulgens Lec.

4723a. obliquus Lec.

4724. otiosus Say.

4724a. pusillus Say.

4724b. defectus Lec.

4725. difficilis Gory.

4726. Walsinghami $\mathrm{Cr}$.

$>4727$. bilineatus $W e b$.

4728. vittaticollis Rand.

4729. granulatus Say.

4730. Lecontei Saund.

4731. fallax Say.

4732. interruptus Lec.

4733. floridanus $\mathrm{Cr}$.

4734. subcinctus Gory.

4735. texanus $\mathrm{Cr}$.

1736. macer Lec.

4737. addendus $\mathrm{Cr}$.

4738. acutipennis Mann.

4739. anxius Gory.

4740. torpidus Lee.

4741. plumbeus Lec.

4742. politus Say.

4743. solitarius Gemm.

4744. cuneus Lec.

4745. imbellis $\mathrm{Cr}$.

4746. egenus Gory.

4746a. cephalicus Lec. 4746b. puncticeps Lec.

4747. lacustris Lec.

4748. putillus Say.

4749. obolinus Lec.

4750. muticus Lec.

4751. pulchellus Bland.

4752. Couesii Lec.

_- lateralis Say.

-- nigricans Gory.

- obsoletoguttatus Gory.

- virens Gory.

\section{Rhæboscelis Chev.}

4753. tenuis Lec.

Taphrocerus Sol.

4751. puncticollis $S z$.

4755. gracilis Say.

4756. agriloides $C r$.

4757. lævicollis $L e c$.

Brachys Sol.

Y 4758. ovata Web.

4758a. lævicauda Lec.

4758b. tessellata Fab.

4759. fascifera $S z$.

4760. prætexta Gory.

4761. ærosa Melsh.

4762. æruginosa Gory.

Pachyscelus Sol.

4763. carbonatus Lec.

ch 4764. purpureus Say.

Ch 4765. cæruleus $S z$.

4766. lævigatus Say.

\section{CA MPY IR IID A.}

Rhyncheros Lec.

4767. Sanguinipennis Say.

Lycus Fab.

4768. eruentus Lec.

4769. Fernandezii Dugès.

\section{Lycostomus Mots.}

4770. Iateralis Melsh.

4771. fulvellus Lec.
4772. loripes Chev.

4773. sanguineus Gorh.

Calopteron Guér.

4774. megalopteron Lec.

4775. terminale Say.

4776. reticulatum Fab.

apicale $L e c$.

typicum te. NEWm

4777. retiferum Lec.

4778, tricarinatum Lec. 
Celetes Newm.

4779. basalis Lec.

Cænia Newm.

4780. dimidiata Fab.

4781. amplicornis Lec.

\section{Lopheros Lec.}

4782. fraternus $R$ and.

\section{Eros Newm.}

4783. thorncicus Rand.

4784. hamatus Mann.

4785. simplicipes Mann.

4756. læetus Mots.

4787. aurora Hbst. coccinatus Say.

4788. mundus Say.

4759. sculptilis Say. oblitus Newm.

4790. humeralis Fab.

4791. trilineatus Melsh.

4792. crenatus Germ.

\section{Plateros Bourg.}

4793. timidus Lec.

4794. modestus Say.

4795. canaliculatus Say.

4796. sollicitus Lec.

4797. lictor Newm. mollis Lec.

4798. Aloralis Melsh.

1799. marginellus $F a b$.

\section{Lygistopterus Muls.}

4800. rubripennis Lec.

4801. lretus Gorh.

Lycaina Dugès.

4802. marginata Gorh.

4803. discoidalis Horn.

Calochromus Guér. Dictyoptera Lat.

4804. fervens Lec.

4805. perfaceta Say.

4806. ruficollis Lec.

4807. dimidiata Lec.
Matheteus Lec.

4808. Therenetii Lec.

Polyclasis Newm.

Calyptocephalus Gray.

4809. bifaria Say.

Lucidota Lap.

6 4810. atra Fab.

tarda Lec.

4811. punctata Lec.

Tenaspis Lec.

4812. angularis Gorh.

Ellychnia Lec.

4813. flavicollis Lec.

4814. californica Mots.

facula Lec.

4815. corrusca Linn. autumnalis Melsh. lacustris Lec.

Pyropyga Mots.

4816. Inteicollis Lec.

4817. fenestralis Melsh. californica Gorh.

4818. nigricans Say.

4819. simplex Lec,

4820. decipiens Harr.

4821. minuta Lec.

4822. indicta Lec.

Pyractomena Lec.

4823. angulata Say. Havocineta Lec.

4824. borealis Rand.

4825. ecostata Lec. nitidiventris Lec.

4826. lucifera Melsh. punctiventris Lec. angustata Lec.

Photinus Lap.

4827. consanguineus Lec. vittiger $\|$ Lec.

4828. lineellus Lec. 
4829. ardens $L e c$.

4830. dimissus Lec.

4831. collustrans Lec.

4832. punctulatus Lec.

4833. umbratus Lec. undulatus err. typ.

4834. benignis $L e c$.

4835. pyralis Linn.

4836. marginellus Lec.

4837. scintillans Say.

\section{Pleotomus Lec.}

4838. pallens Lec.

4839. nigripennis Lec.

4840. Davisii Lec.

\section{Lamprohiza Mots.} Phausis Lee.

4841. splendidula Linn.

4842. reticulata Say.

4843. inaceensa Lec.

4844. Riversi Lec.

\section{Microphotus Lec.}

4845. dilatatus Lec.

4846. angustus Lec.

Photuris Lec.

4847. pennsylvanica $D e G$. 4848. frontalis Lec.

4849. divisa Lec.

\section{Pterotus Lec.}

4850. obscuripennis Lec.

\section{Phengodes Ill.}

4851. frontalis Lec.

4852. plumosa Oliv.

4853. laticollis Lec.

4854. fusciceps Lec.

4855. Sallei Lec.

\section{Zarhipis Lee.}

4856. integripennis $L e c$.

4857. Riversi Horn.

485. ruficollis Lec.

4859. piciventris Lec.
Mastinocerus Sol.

4860. texanus Lec.

\section{Cenophengus Lec.}

4861. debilis Lec.

\section{Spathizus Lec.}

4862. bicolor Lec.

\section{Tytthonyx Lec.}

4863. erythrocephalus Fab.

\section{Omethes Lec.}

4864. marginatus Lec.

\section{Chauliognathus Hentz.}

4865. profundus thec.

4866. ineptus Horn.

4867. discus Lec.

4868. misellus Horn.

4869. fasciatus Lec.

4870. limbicollis Lec.

4871. basalis Lec.

4872. scutellaris Lec.

4873. Lewisii $C r$.

4874. opacus Lec.

4875. pennsylvanicus $D e G$.

7876. marginatus $F a b$.

\section{Podabrus Westw.}

4877. latimanus Mots.

4878. nothoides Lec.

4879. tricostatus Say.

4880. rugulosus Lec.

4881. frater Lec.

4882. basilaris Say. discoideus Lec. flavicollis $L e c$. punctulatus Lec.

4883. quadratus Lec.

4884. fissus Lec.

4885. diadema Fab.

4886. modestus Say.

4887. binotatus Lec.

488s. comes Lec. gradatus Lec.

$\grave{\nu} 4889$. tomentosus Say. pruinosus Lec. 
4890. protensus Lec. Fayi Lec.

4591. brunnicollis Lec.

4\$92. scaber Lec.

4893. cinctipennis Lec.

4594. limbellus Lec.

4\$95. punctatus Lec.

4896. brevipennis Lec.

4897. puncticollis Kirby.

4898. xanthoderus Lec.

4599. Iutosus Lec.

4900. macer Lec.

4901. piniphilus Esch.

4002. lateralis Lec.

4903. puberulus Lec.

4904. extremus Lec.

4905. simplex Coup.

4 4906. lrvicollis Kirby.

4907. tejonicus Lec.

4908. Pattoni Lec.

4909. Bolteri Lec.

4910. mellitus Lec.

4911. corneus Lec.

4912. cavicollis Lec.

Silis Lat.

4913. spinigera Lcc.

4914. munita Lec.

4915. difficilis Lec.

4916. flavida Lcc.

4917. cava Lcc.

4918. atra Lec.

4919. pallida Mann.

4920. percomis Say.

4921. vulnerata Lec.

4922. lutea Lec.

4923. filigera Lec.

4924. spathulata Lec.

4925. perforata Lcc.

\section{Telephorus Schätt.}

4926. dentiger Lec.

4927. excavatus Lec.

4028. vilis Lec.

4929. tantillus Lec.

4930. Walshii Lec.

4931. fraxini Say.

44932. carolinus Fab.

4933. lineola Fab. angulatus Say.
4934. costipennis Lec.

4935. nigritulus Lec.

4936. rectus $\mathrm{Melsh}$.

oriflavus Lec.

pusillus Lec.

4937. nanulus Lec.

4938. cruralis Lec.

4939. Havipes Lec.

? dichrous Lec.

4940. scitulus Say.

imbecillis Lec.

nigriceps Lec.

4941. pusillus Lec.

4942. Iuteicollis Germ.

4943. ruficollis Lec.

4944. longulus Lcc.

4945. impar Lec."

4946. consors Lec.

tibialis Lec.

4947. rotundicollis Say.

4948. Curtisii Kirby.

Samouelli Kirby.

? Westwoodii Kirby.

4949. transmarinus Mots.

4950. grandicollis Lec.

4951. fidelis Lec.

4952. oregonus Lec.

scopus Lec.

4953. tuberculatus Lec.

armiger Coup.

collaris || Lec.

4954. alticola Lec.

4955. bilineatus Say.

4056. divisus Lec.

4957. notatus Mann.

peregrinus Boh.

4058. lautus Lec.

4959. ochropus Lec.

4960. ingenuus Lec.

4961. marginellus Lec.

... invalidus $\mathbf{S} a y$.

_- rufipes Say.

- ? vituata $F a b$.

Polemius Lec.

4962. princeps $L \in c$.

4963. strenuus Lec.

4964. marginicollis Lec.

4965. platyderus $G$. \& $H$. planicollis \| Lec. 
4966. laticornis Say. incisus Lec.

4967. undulatus Lec. 4968. limbatus Lec.

\section{Ditemnus Lec.}

4969. bidentatus Say.

4970. obtusus Lec.

1971. fossiger Lec.

\section{Trypherus Lec.}

1972. latipennis Germ.

Lobetus Kies.

4973. abdominalis Lec.

\section{Malthinus Iat.}

4974. atripennis Lec.

4975. occipitalis Lec. difficilis $L e c$.
Malthodes Kies.

4976. bicolor Lec.

4977. spado Lec.

4978. laticollis Lec.

transversus $\|$ Lec.

4979. concavus Lec.

4980. fragilis Lec.

4981. exilis Melsh.

4982. fusculus Lec.

4983. rectus Lec.

4984. eurvatus Lec.

4985. furcifer Lec.

4986. arcifer Lec.

4987. captiosus Lec.

4988. fuliginosus $L e c$.

4989. niger Lec.

4990. analis Lec.

4991. congruus Lec.

4992. quadricollis Lec.

4993. parvulus Lec.

-_ ? marginipennis Lap.

IALACIID E.

\section{Collops Er.}

7 4994. tricolor Say.

4995. punctatus Lec.

4996. eximius Er.

4997. marginicollis Lec.

4998. nigriceps Say.

4999. cribrosus Lec.

5000. reflexus Lec.

5001. hirtellus Lec.

5002. bipunctatus Say.

5003. validus Horn.

> 5004. 4-maculatus Fab.

5005. histrio $E r$.

5006. pulchellus Horn.

5007. balteatus Lec.

5008. insulatus Lec.

5009. limbellus G. \& $H$.

5010. laticollis Horn.

5011. confluens Lec.

5012. punctulatus Lec.

$>$ 5013. vittatus Say.

5014. marginellus Lec.

\section{Temnopsophus Horn.}

5015. bimaculatus Forn.

5016. impressus $S z$.
Trophimus Horn.

5017. æneipennis Horn.

Endeodes Lec.

5018. basalis Lec.

5019. abdominalis Ler.

5020. collaris Lec.

Chretoccelus Lec.

5021. setosus Iec.

\section{Malachius Fab.}

5022. æneus Linn.

5033 . biguttulus Horn.

5024. anritus Lec.

5025. spinipennis Horn.

5026. mirandus Lec.

5027. Thevenetii Horn.

5028. macer Horn.

5029. mixtus Horn.

5030. Ulkei Horn.

5031. montanus Lec.

Tanaops Lec.

5032. abdominalis Lec.

5033. longiceps Lec. 
Microlipus Lec.

5034. laticeps Lec.

5035. lævicollis Horn.

5036. mørens Lec.

5037. Longicollis Mots.

\section{Anthocomus Er.}

5038. Erichsoni Lec.

5039. Aavilabris Say.

5040. ventralis Horn.

\section{Pseudebrus Horn.}

5041. apicalis Say.

5042. bicolor Lec.

5043. pusillus Say.

5044. obscurus Lec.

5045. oblitus Lec.

- ? labiatus $F a b$.

\section{Attalus Er.}

5046. rostratus Horn.
5047. trimaculatus Mots.
5048. rufomarginatus Mots.
5049. oregonensis Horn.
5050. nigrellus Lec.
5051. basalis Lec.
5052. terminalis Er.
5053. varians Horn.
5054. granularis Er.
5055. morulus Lec.
5056. pallifrons Mots.
5057. melanopterus Er.
5058. humeralis Lec.
5059. parallelus Horn.
5060. Pettiti Horn.
5061. rufiventris Horn.
5062 . nigripes Horn.
5063. otiosus Say.
5064. circumseriptus Say.
5065 . cinctus Lec.
5066 . difficilis Lec.
5067. lobulatus Lec.
5068 . scincetus Say.
- ? submarginatus Lec.

Pristoscelis Lec.

5069. rufipes Mots.

5070 . brevicornis Lec.

5071. fuscus Lec.
5072. ater Bland.

5073. oregonensis Lec.

5074. laticollis Mann.

5075. fulvotarsis Bland.

5076. atricornis Lec.

5077. convergens Lec.

5078. umbratus Lec.

5079. antennatus Mots.

5080. erythropus Lec.

5081. californicus Mots.

5082. eylindricus Mots.

5083. brevipilosus Lec.

5084. hirtellus Lec.

5085. sordidus Lec.

5086. suturalis Lec.

5087. conformis Lec.

5088. quadricollis Lec.

5089 . tejonicus Lec.

5090. squalidus Lec.

5091. cruralis Lec.

5092. ænescens Lec.

5093. punctipennis Lec.

5094. grandiceps Lec.

5095. pedalis Lec.

5096. texanus Lec.

5097. serricollis Lec.

5098. serrulatus Lec.

5099. rufipennis Lec.

5100. comatus Lec.

_ ? parvicollis Mann.

\section{Listrus Mots.}

5101. Motschulskii Lec.

5102. interruptus Lec.

5103. canescens Mann.

5104. difficilis Lec.

5105 . rotundicollis Lec.

5106. obscurellus Lec.

5107. luteipes Lec.

5108. tibialis Mots.

5109. senilis Lec.

\section{Dolichosoma Steph.}

5110. foveicolle Kirby.

5111. nigricorne Bland.

5112. tenuiforme Horn.

Eschatocrepis Lec.

5113. constrictus Lec. 
Allonyx Lec.

5114. sculptilis Lec.

5115. plumbeus Lec.

\section{Dasytes Payk.}

5116. breviusculus Mots.

5117. hudsonicus Lec.

5118. seminudus Lec.

5119. pusillus $L e c$.

5120. ruficollis Ulke.
Mecomycter Horn.

5121. omalinus Horn.

Melyris Fab.

5122. basalis Lec.

5123. cribrata Lec.

5124. atra Lec.

5125. flavipes Lec.

Rhadalus Lec.

5126. testaceus Lec.

\section{$\checkmark$ CLERIDE.}

\section{Elasmocerus Lec.}

5127. terminatus Say.

\section{Tillus Oliv.}

5128. collaris Spin.

5129. occidentalis Gorh.

\section{Cymatodera Gray.}

5130. longieornis Lec.

5131. puncticollis Bland.

5132. turbata Horn.

5133. Xanti Horn.

5134. cylindricollis Chev. usta Lec.

5135. brunnea Melsh.

5136. bicolor Say.

5137. inornata Say. tenera Lec.

5138. fascifera $L e c$.

5139. californica Horn.

5140. Hopei Gray. gigantea Horn.

5141. Belfragei Horn.

5142. morosa Lec.

5143. punctata Lec.

5144. fuscula Lec.

5145. texana Gorh.

5146. oblita Horn.

Ch 5147. undulata Say. $5147 a$. balteata Lec.

5148. sirpata Horn.

5149. fallax Horn.

5150. angustata Spin.

5151. ovipennis Lec.

5151a. pilosella Lec.

\section{Priocera Lec.}

5152. castanea Newm.

\section{Opilus Lat.}

5153. domesticus $K l$.

Tarsostenus Spin.

5154. univittatus Rossi.

Aulicus Spin.

5155. Nero Spin.

Trogodendron fform. Guer

5156. Edwardsii Horn.

Trichodes Hbst.

5157. illustris Horn.

C 5158. ornatus Say.

$5158 \alpha$. tenellus Lec.

c) 5159. Nuttalli Kirby.

5160. bimaculatus Lec.

5161. apivorus Germ.

5162. simulator Horn.

c 5163. bibalteatus Lec.

Clerus Geoff.

Cleronomus $\mathrm{KI}$.

5164. Spinolæ Lec.

5165. quadrisignatus Say.

5166. analis Lec.

$>$ 5167. quadriguttatus Oliv. nigrifrons $S a y$. nigripes Say.

5168. ocreatus Horn. 
C. 5169 . rosmarus Say.

5170. lunatus Spin.

5171. ichneumoneus $F a b$.

5172. decussatus $K l$.

5173. abruptus Lec.

5174. crabronarius Spin.

5175. cordifer Lec.

5176. eximius Mann.

5177. nigriventris Lec.

5178. sphegeus $F a b$. arachnodes $K l$.

5179. mostus $K l$.

5180. thoracicus Oliv. ornaticollis Lec.

5181. sexguttatus $F a b$.

Thanasimus Lat.

5182. trifasciatus Say. 5183. repandus Horn.

$\checkmark$ 5184. dubius $F a b$.

$5184 a$. rubriventris Lec.

5185. undulatus Say.

$5185 a$. nubilus $K l$.

\section{Thaneroclerus Spin.}

Yे 5186. sanguineus Say. 5187. tantillus Lec.

\section{Hydnocera Newm.}

5188. tricondylæ Lec.

5189. unifasciata Say.

5190. robusta Horn.

5191. subfasciata Lec.

5192. subænea Spin.

c 5193. humeralis Say.

5193a. cyanescens Lec.

5193b. diftieilis Lec.

5194. rufipes Newm.

5195. pubescens Lec.

5196. seabra $L e c$.

5197. discoidea Lec.

5198. hamata Lec.

C 5199. pallipennis Say.

5200. suturalis $K l$.

5201. bicolor Lcc.

5202. pedalis Lec.

i203. verticalis Say.

5204. Schusteri Lec.

5205. tabida Lec.

5206. longicollis Zicgl.
5207. longa Lec. 5208. ægra Newm.

5209 . albocincta Horn.

Phyllobænus Spin.

ch 5210. dislocatus Say.

Ichnea Lap.

5211. laticornis Say.

Chariessa Perty.

5212. vestita Spin.

5213. elegans Horn.

5214. dichroa Lec.

5215. pilosa Forst.

5215a. onusta Say.

Cregya Lec.

5216. vetusta Spin.

5217. fasciata Lec.

5218. oculata Say.

5219. mixta Lec.

\section{Enoplium Lat.}

5220. quadripunctatum Say.

5221. quadrinotatum Hald.

5222. scabripenne Lec.

5223. humerale Horn.

Orthopleura Spin.

5224. texana Bland.

5225. damicornis Fab.

Lebasiella Spin.

5220. janthina Lec.

i227. maculicollis Lec.

5228. discoidea Lec.

Laricobius Rosen.

5229. Erichsoni Rosen. rubidus Lec.

Necrobia Lat.

$\forall$ 5230. rufipes Fab.

y 5231. ruficollis Fab.

C. 5232. violaceus Linn.

5233. marginellus Chev.

\section{Opetiopalpus Spin.}

5234. luridus Spin. 
P'TINIDA.

- Gibbium Scop.

5235. scotias Scop.

Mezium Curt.

5236. americanum Lap.

Trigonogenius Sol.

5237. farctus Lec.

Niptus Boieldieu.

5238. ventriculus $L e c$.

Ptinus Linn.

5239. fur Linn.

5240. brunneus Duft.

5241. verticalis Lee.

5242. quadrimaculatus $\mathrm{Melsh}$.

5243. bimaculatus Melsh.

5244. interruptus Lec.

\section{Eucrada Lec.}

5245. humeralis Melsh.

Hedobia Lat.

5246. granosa Lec.

Ernobius Thom.

5247. mollis Linn.

5248. punctulatus Lec.

5249. alutaceus Lec.

5250. debilis Lec.

5251. granulatus Lec.

5252. marginicollis Lec.

5253. tenuicornis Lec.

5254. luteipennis Lec.

5255. filicornis Lec.

5256. tristis Lec.

5257. gracilis Lec.

\section{Ozognathus Lec.}

5258. cornutus Lec.

5259. floridanus Lec.

5260. misellus Lec.

Xestobium Mots.

5261. tesselatum $\mathrm{Fab}$.
5262. squalidum Lec.

5263. affine Lec.

Oligomerus Redt.

5264. sericans Melsh.

5265. obtusus Lec.

5266. alternatus Lec.

Sitodrepa Thom.

Y 5267. panicea Linn.

Ctenobium Lec.

5268. antennatum Lec.

Ptinodes Lec.

5269. setifer Lec.

Hadrobregmus Thom.

$>5270$. errans Melsh.

5271. carinatus Say.

5272. linearis Lec.

5273. pumilio Lec.

5274. gibbicollis Lec.

5275. foveatus Kirby.

Gastrallus Duval.

5276. marginipennis Lec.

Trichodesma Lee.

5277. gibbosa Say.

Nicobium Lec.

5278. hirtum Ill.

Anobium Fab.

5279. notatum Say.

5280. quadrulum Lec.

Trypopitys Redt.

5281. sericeus Say.

5282. punctatus Lec.

Petalium Lec.

5283. bistriatum Say. 
Theca Muls.

5284. profunda Lec.

5285. striatopunctata Lec.

\section{Eupactus Lec.}

5286. nitidus Lee.

5287. viticola $S z$.

5288. punctulatus Lec.

_- ? pudieus' Boh.

Vrilletta Lec.

5289. convexa Lec.

5290. expansa Lec.

5291. Murrayi Lec.

Xyletinus Lat.

5292. peltatus Harr.

5293. pallidus Lec.

5294. mucoreus Lec.

5295. fucatus Lec.

5296. lugubris Lec.

5297. puberulus Boh.

5298. pubescens Lec.

Lasioderma Steph.

5299. serricorne Fab. ? castaneum Melsh.

5300. dermestinum Lec.

\section{Catorama Guér.}

5301. frontalis Lec.

5302. simplex Lec.

5303. holosericea Lec.

5304. sectans Lec.

5305. punctulata Lec.

5306. obsoleta Lec.

5307. minuta Lec.

-_ ? tabaei Guér.

\section{Hemiptychus Lec.}

5308. punctatus Lec.
5309 . gravis Lec.
5310 . pusillus Lec.
5311 . borealis Lec.
5312. ventralis Lec.
5313. debilis Lec.
5314. similis Lec.

5315. abbreviatus Lec.

5316. auctus Lec.

5317. obsoletus Lec.

5318. nigritulus Lec.

\section{Protheca Lec.}

5319. puberula Lec.

5320. hispida Lec.

\section{Dorcatoma Hbst.}

5321. setulosum Lec.

5322. incomptum Lec.

5323. pallicorne Lec.

5324. granum Lec.

5325. tristriatum Lec.

\section{Cænocara Thom.}

5326. oculata Say.

5327. californica Lec.

5328. lateralis Lec.

5329. seymnoides Lec.

5330. intermedia Lec.

-_ ? bicolor Germ.

Byrrhodes Lec.

5331. setosus Lec.

Ptilinus Geoff.

5332. ruficornis Say.

5333. basalis Lec.

5334. thoracicus Rand.

\section{Euceratocerus Lec.}

5335. Hornii Lec.

\section{Endecatomus Mellí́.}

5336. reticulatus Host.

5337. rugosus Rand.

Sinoxylon Duft.

5338. simplex Horn.

5339. basilare Say.

5340. sericans Lec.

5341. toridanum Horn.

5342. texanum Horn.

5343. sextuberculatum Lec.

5344. quadrispinosum $L e c$. 
5345. dinoderoides Horn.

5346. bidentatum Horn.

5347. declive Lec.

5348. suturale Horn.

\section{Tetrapriocera Horn.}

5349. longicornis Oliv.

\section{Bostrychus Geoff.}

5350. bicornis Web.

5351. armiger Lec.

5352. truncaticollis Lec.

5353. californicus Horn.

Amphicerus Lec.

5354. fortis Lec.

5355. punctipennis Lec.

5356. bicaudatus Say.

5357. teres Horn.

Dinoderus Steph.

5358. porcatus Lec.

5359. substriatus Payk.

5360. cribratus Lec.

5:61. densus Lec.

5362. pusillus $F a b$.

5363. punctatus Say.
5364. truncatus Horn.

5365. brevis Horn.

\section{Polycaon Lap.}

5366. Stoutii Lec.

5367. punctatus Lec.

5368. exesus Lec.

5369. obliquus Lec.

5370. plicatus Lec.

5371. confertus Lec.

Psoa Hbst.

5372. maculata Lec.

5373. 4-signata Horn.

Lyctus Fab.

Bitoma Hbst.

5374. striatus Melsh.

5375. opaculus Lec.

parvulus Casey.

5376. planicollis Lec.

Trogoxylon Lec.

5377. californicum $\mathrm{Cr}$.

5378. parallelopipedum Melsh.

5379. punctatum Lec.

\section{CUPESID A.}

Priacma Lec.

5380. serrata Lec.

5382. concolor Westwo.

5383. capitata Fab.

\section{Cupes Fab.}

5381. lobiceps Lec.

\section{LYMEXYLIDA。}

Hylecœtus Lat.

5384. lugubris Say.

Lymexylon Fab.

5385. sericeum Harr.
Micromalthus Iec.

5386. debilis Lec.

\section{CIOID $A$.}

Cis Lat.

5387. dichrous Lec.

5388. creberrimus Mellié.

5389. fuscipes Mellié.
5390. americanus Mann.

5391. bicarinatus Mann.

5392. tridentatus Mann.

5393. Chevrolatii Mellié. 
5394. setulosus Mellié.

5395. atripennis Mellié.

5396. dubius Mellié.

5397. pumicatus Mellié.

5398. minutissimus Mellié.

5399. obesus Mellié.

5400 . punctatus Mellié.

5401. tristis Mellié.

5402. subtilis Mellié.

Ennearthron Mellié.

5403. vitulus $\operatorname{Mann}$.
5404. thoracicornis Ziegl. Mellyi Mellié. unicolor Casey. 5405. californicus Casey.

Ceracis Mellié.

5406. Sallei Mellié.

Rhipidandrus Lec.

5407. paradoxus Beauv.

\section{SIIIIN DIDE.}

Odontosphindus Lec.

5408. denticollis Lec.

Sphindus Chev.

Eurysphindus Lec.

5410. hirtus Lec.

5409. americanus Lec.

\section{LUCANIDE.}

Lucanus Linn.

5111. elaphus Fab.

Oh 5112. dama Thunb.

7 5413. mazama Lec.

Ch 5414. placidus Say.

Dorcus Mact.

>5415. parallelus Say. 5415a. costatus Lec. 5415b. brevis Say.

Platycerus Geofl:

C5416. quercus $W_{\epsilon} b$. 5417. oregonensis Westw.
5418. Agassii Lec.

5419. depressus Lec.

Ceruchus MacL.

5420. punctatus Lec.

5421. striatus Lec.

\& 5422. piceus $W c b$.

Sinodendron Hellw.

5423. rugosum $\operatorname{Mann}$.

Passalus Fab.

Ch 5424. cornutus Fab.

\section{$\int$ SCAIRAIBAIDA.}

Canthon Hoftim.

5425. nigricornis Say.

c 5426. ebenus Say.

5427. depressipennis Lec.

5428. praticola Lec.

5429. probus Germ.

5430. puncticollis Lec.

5431. Lecontei Harold.
5432. simplex Lec. 5432a. corvinus Horn. 5432b. militaris Horn. 5432c, humeralis Horn. 5433. cyanellus Lec. 5434. vigilans $L e c$. 5435. lævis Drury. hudsonias Forst. 
5436. chalcites Hald.

5437. indigaceus Lec.

5438. viridis Beruv.

5439. perplexus Lec.

\section{Deltochilum Esch.}

5440. gibbosum Fab.

\section{Chœridium Lep.}

5441. histeroides $W e b$.

5442. Lecontei Harold.

\section{Copris Geoff:}

5443. minutus Drury.

(C) 5444. anaglypticus Say.

5445. remotus Lec.

5446. moeluus Lec.

5447. procidua Say.

$>$ 5448. carolina Linn.

Phanzus MacL.

5149. pluto Harold.

5450. quadridens Say.

C 5451. difformis Lec.

C 5452. carnifex Linn.

5453. triangularis Say.

5454. igneus MacL.

nigrocyaneus MacL.

5455. mexicanus Harold.

\section{Onthophagus tat.}

5456. nuchicornis Linn.

5457. coproides Horn.

† 5458. Hecate Panz.

\. 5459. Janus Panz.

5459a. Orpheus Panz.

5459b. striatulus Beauv.

5459c. subreneus Beauv.

5460 . brevifrons Horn.

5461. velutinus Horn.

5462. tuberculifrons Harold.

ह

5463. pennsylvanicus Harold.

5464. cribricollis Horn.

\section{Oniticellus Ziegl.}

5465. californicus Horn.

\section{Aegialia Lat.}

5466. rufa $L e c$.
5467. cylindrica Esch.

5468. lacustris Lec.

5469. conferta Horn.

5470. latispina Lec.

5471. crassa Lec.

5472. spissipes Lec.

\section{Psammodius Heer.}

5473. ægialioides Hald.

5474. 5-plicatus Horn.

5475. interruptus Say.

5476. crelatus Lec.

5477. bidens Horn.

5478. clypeatus Say.

Pleurophorus Muls.

5479. crsus Panz.

\section{Rhyssemus Muls.}

5480. scaber Hald.

5481. ealifornieus Horn.

5482. sonatus Lec.

5493. riparius Horn.

5484. cælatus Lec.

Euparia Serv.

5485. castanea Serv.

Atænius Harold.

5486. imbricatus Melsh.

5487. alternatus Melsh.

5488. robustus Horn.

5489. oblongus Horn.

5490. figurator Har.

5491. gracilis Melsh.

5492. ovatulus Horn.

5493. stercorator $F a b$.

5494. lobatus Horn.

5495. socialis Horn.

5496. puncticollis Lec.

5497. hirsutus Horn.

5498. lucanus Horn.

5499. abditus Hald.

5500. sculptilis Har.

5501. cylindrus Horn.

5502. desertus Horn.

Dialytes Harold.

5503. truncatus Melsh. 
5504. striatulus Say.

5505. Ulkei Horn.

\section{Oxyomus Lap.}

5506. porcatus Fab.

\section{Aphodius Ill.}

5507. fossor Linn.

5508. pinguis Hald.

5509. validus Horn.

5510. hamatus Say.

5511. hyperboreus Lec.

5512. torpidus Horn.

5513. occidentalis Horn.

5514. erraticus Linn.

5515. denticulatus Hald.

5516. obtusus Lec.

5517. fimetarius Linn.

5518. guttatus Esch.

5519. aleutus Esch.

5520, bidens Lec.

5521. crassulus Horn.

5) 22. ursinus Mots.

552:. ruricola Melsh.

5i24. congregatus Mann.

ji2j. footidus Fab.

5.521. pectoralis Lec.

5,227. duplex Lec.

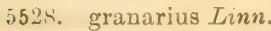

539. vittatus Say.

ji:n. nevadensis Horn.

5531. cribratus Lec.

5532. rugifrons Horn.

5533. lividus Oliv.

5534. vestiarius Horn.

55.35. serval Say.

55.36. inquinatus Hbst.

' 55:37. pardalis Lec.

j.i:- leopardus Horn.

5i:?. anthracinus Lec.

540 . explanatus Lec.

i.j.1. opacus Lec.

55.12. lutulentus Hald.

554.). stupidus Horn.

5544 , lentus Horn.

5.54. rubenlus Beauv.

5.546. stercorosus Melsh.

5547 . militaris Lec.

5.54. rubiginosus Horn.

5549. xgrotus Horn.
5550. consentaneus Lec.

5551. politus Horn.

5552. brevicollis Lec.

5553. marginatus Lec.

5554. phæopterus Lec.

555. cruentatus Lec.

5).j6. subtruncatus Lec.

5.57\%. scabriceps Lec.

$555 \%$. ochreipennis Horn.

5.5!). rubidus Lec.

5360 . concavus Say.

5.561 . subreneus Lec.

5.)62. alternatus Horn.

jobis. terminalis Say.

5.tit. coloradensis Horn.

5.565. bicolor Say.

j5liti. dentiger Lec.

556\%. phalerioides Horn.

.569. < prodromus Brahm.

5.56!. femoralis Say.

5570. Walshii Horn.

5571 . rubripennis Horn.

5572. oblongus Say.

557:. cadaverinus Mann.

5,74. rudis Lec.

5575 . sparsus Lec.

jiiti. humeralis Lec.

Ochodæus Serv.

5.77. pectoralis Lec.

5i): musculus Say.

5579. simplex Lec.

5). Ulke. Ulkern.

jis1. biarmatus Lec.

$5.5,2$. frontalis Lec.

5i) 4.). striatus Lec.

5iti. sparsus Lec.

- - duplex Lec.

\section{Hybosorus MacL.}

5585. Illigeri Reiche.

\section{Pachyplectrus Lec.}

5586. lrevis Lec.

\section{Bradycinetus Horn.}

5587. ferrugineus Beauv.

5588. fossator Hald.

5589. serratus Lec. 
Bolboceras Kirby.

5590. faretus $F a b$.

$5.590 a$. tumefactus Beauv.

5591. lazarus $F a b$.

\section{Odontæus IKl.}

5592. filieornis Say.

5593. cornigerus Melsh.

5594. obesus Lec.

\section{Geotrupes Lat.}

5595. retusus Lec.

$\&$ 5596. splendidus Fab.

Y 5597. semiopacus Jek.

5598. chalybrus Lec.

5599. necidentalis Horn.

5600. Egeriei Germ.

5601. opacus Hald.

5602. Blackburnii Fab.

5603. Balyi Jek.

Pleocoma Lec.

C. 5604. fimbriata Lec. 5605. Behrensii Lec. 5006. hirticollis Schurf. 5607. Edwardsii Lec.

\section{Clœotus Germ.}

5608. aphodioides IIl. 5609. globosus Say.

Sphæromorphus Germ. Acanthocerus MacL.

5610. renens MacL.

\section{Nicagus Lec.}

5611. obseurus Lec.

\section{Trox Fab.}

5612. scutellaris Say. ifil3. seabrosus Beauv.

5614. monachus Hbst. pustulatus Lec.

5615: asper Lec.

5616. suberosus $F a b$.

5617. punctatus Germ.

5618. tuberculatus DeG.
5619. gemmulatus Horn.

5620. sonora Lec.

5621. erinaceus Loc.

5622. capillaris Say.

5623. unistriatus Beauv.

5624 . sordidus Lec.

5625. foveicollis Har.

Z 5626. terrestris Say.

\& 5627. xqualis Say.

5628. fascifer Lec.

5629. scaber Linn.

5630 . atrox Lcc.

5631. laticollis Lec.

5632. striatus Melsh.

\section{Glaresis Er.}

5633. mendica Horn.

5634 inductr Horn.

\section{Amphicoma Lat.}

Dasydera Lec.

Lichnanthe Burm.

5635. Iupina Lec.

5636. ursina Lec. Cooperi Horn.

5637. vulpina Hentz.

5638. Edwardsii Horn.

5639. canina Horn.

5640. Rathvoni Lec.

Podolasia Harold.

5641. ferruginea Lec.

\section{Oncerus Lec.}

5642. foralis Lec.

\section{Chnaunanthus Burm.}

Acratus Horn.

5643. flavipennis Horn.

Hoplia III.

5614. Sackenii Lec.

5645. callipyge $\mathrm{Lec}$.

5646. hirta Lec.

5647. pubicollis $L \mathrm{cc}$.

5648. laticollis Lec.

5649. dispar Lec.

5650. trifasciata Say. 
$5650 \%$ tristis Melsh.

5651. trivialis Harold.

5652. mucorea Germ.

5653. limbata Lec.

5654. modesta Hald. singularis Burm.

5655. equina Lec.

\section{Dichelonycha Kirby.}

- $\$$ 5656. elongata Fab.

5657. subvittata Lec.

5658. canadensis Horn.

5659. testacea Kirby.

5660. pallens Lec.

5661. Crotehii Horn.

5662. Backii Kirby.

5663. fuseula $L e c$.

5664. fulgida $L c c$.

5665. truncata Lec.

5666. elypeata Horn.

خ 5667. albicollis Burm.

5668. valida Lec.

5669. suleata Lec.

5670. pusilla Lec.

\section{Cœnonycha Horn.}

5671. rotundata Lec.

5672. socialis Horn.

5673. ovipennis Horn.

Serica MacL.

Sy 5674. vespertina Gyll.

5675. texana Lec.

5676. atratula Lec.

5677. serotina Lec.

5678. iricolor Say.

5679. fimbriata Lec.

5680. tristis Lec.

5681. sericea Ill. parallela Casey.

5682. curvata Lec.

5683. elongatula Horn. porcula Casey.

5684. mixta Lec.

5685. alternata Lec.

5686. anthracina Lec.

5687. frontalis Lec.

5688. valida $H a r$.

5689. trociformis Burm.
Macrodactylus Lat.

A 5690. subspinosus Fab. 5691. ingustatus Beauv. 5692. uniformis Horn.

\section{Hypotrichia Lee.}

5693. spissipes Lec.

\section{Plectrodes Horn.}

5694. pubescens Horn.

5695. Carpenteri Lec.

5696. palpalis Horn.

\section{Orsonyx Lec.}

5697. anxius Lec.

\section{Diazus Lec.}

5698. rudis Lec.

Diplotaxis Kirby.

5699. sordida Say.

5700. puberula Lec.

5701. popino Casey.

5702. subcostata Blanch.

5703. liberta Germ.

5704. brevicollis Lec.

5705. obscura Lec.

5706. tristis Kirby.

5707. excavata Lec.

5707a. frondicola $\ddagger$ Blanch.

5708. frontalis Lec.

5709. georgiæ Blanch.

5710. eastanea Burm.

5711. corpulenta Burm.

5712. angularis Lec.

5713. mœrens Lec.

5714. punctipennis Lec.

5715. texana Lec.

5716. Harperi Blanch.

5717. frondicola Say.

5718. dubia Lec.

5719. truncatula Lec.

5720 . consors Lec.

5721. carbonata Lec.

5722. atratula Lec.

5723. morula Lec.

5724. punctata Lec. levicula Casey. 
5725. cribulosa Lec.

5726. subangulata Lec.

5727. bidentata Lec.

5728. languida Lec.

5729. tenuis Lec.

.5730. corvina Lee.

5731. pacata Lec.

5732. brevidens Lec.

5733. Haydenii Lec.

5734. insignis Lec.

5735. innoxia Lec.

\section{Alobus Lec.}

5736. fulvus Lec.

\section{Lachnosterna Hope.}

\section{7. cribrosa Lec.}

5738. ventricosa Lec.

5739. lanceolata Say.

5740. æqualis Lec.

5741. fureta Lec.

5742. torta Lec.

5743. frontalis Lec.

5744. longitarsus Say.

? gracilis $B$ urm.

5745. dispar Burm.

5746. debilis Lec.

5747. latifrons Lee.

C 5748. cerasina Lec.

? prununculina Burm.

5749. ephelida Say.

5750. uniformis Blanch.

5751. Burmeisteri Lec.

5752. glaberrima Blanch.

5753. inana Lec.

5754. volvula Lec.

5755. congrua Lec.

5756. futilis Lec.

5757. fusea Fröh.

5757a. "consimilis Lec.

5757b. anxia Lec.

5757 c. brevicollis Burm.

5757d. puncticollis Blanch.

5757e. Drakii Kirby.

5758. eephalica Lec.

5759. errans Lec.

5760. decidua Lec.

5761. micans Knoch.

5762. subpruinosa Casey.
5763. serricornis Lec.

5764. semicribrata Lec.

5765. lugubris Lec.

5766. cognata Burm.

5767. fraterna Harr.

5768. lutescens Lec.

5769. corrosa Lec.

5770. ealceata Lec.

5771. marginalis Lec.

Ch 5772. crassissima Blanch.

5773. prunina Lec.

5774. rugosa Melsh.

5775. affinis Lec.

5776. Knochii Sch. \& Gyll.

5777. ilicis Knoch.

5778. ciliata Lec.

- - ilicis $\ddagger$ Burm.

5779. subtonsa Lec.

5780. hirticula Knoch.

5781. hirsuta Knoch.

5782. balia Say.

5783. villifrons Lec.

5784. hirticeps Lec.

5785. nitida Lec.

5786. rufiola Lec.

5787. integra Lec.

5788. quercus Knoch.

- longicornis Blanch.

- diffinis Blanch.

- gibbosa Burm.

- Forsteri Burm.

5789. crenulata Fröh.

5790. albina Burm.

5791. parvidens Lec.

5792. rubiginosa Lec.

5793. submucida Lec.

5794. glabricula Lec.

5795. glabripennis Lec.

$>$ 5796. tristis Fab.

5797. crinita Burm.

_- comans Burm.

5798. maculicollis Lec.

5799. nitidula Lec.

Phytalus Er.

5800. robustus Horn.

5801. cephalicus Horn.

5802. pallidus Horn.

5803. vexatus Horn.

5804. debilis Horn.

5805. georgianus Horn. 
Listrochelus Blanch.

5806. disparilis Horn.

5807. flavipennis Horn.

5808. puberulus Lec.

5809. ecoparius Lec.

5810. gracilis Horn.

5811. densicollis Lec.

5812. mucoreus Lec.

5813. obtusus Lec.

5814. opacicollis Horn.

5815. sociatus Horn.

5816. timidus Horn.

5817. falsus $L e c$.

5818. fimbripes Lec.

5819. senex Horn.

\section{Polyphylla Harr.}

5820. Hammondi Lec. subvittata Lec.

5821. cavifrons Lec.

y 5822. decemlineata Say.

C.5823. crinita Lec,

5\$24. occidentalis Linn.

5825. variolosa Hentz.

5826. graeilis Horn.

Thyce Lec.

5827. squamicollis Lec.

\section{Phobetus Lec.}

5828. comatus Lec.

Anomala Koeppe.

5829. parvula Burm.

5830. flavipennis Burm.

5831. binotata Gyll. luteipennis Lec.

5832. centralis Lec.

Y 5833. minuta Burm.

5834. undulata Melsh. varians $\|$ Fab.

5835. lurida $F a b$. inconstans Burm.

5836. semilivida Lec.

5837. eavifrons Lec.

5838. lucicola $F a b$.

5839. oblivia Horn.

5840. marginata $F a b$.
Strigoderma Burm.

Y. 5S41. pygmæa Fab.

5842. arboricola $F a b$. pimalis Casey.

5843. exigua $\$ \mathbf{z}$.

Pelidnota MacL.

(1) 5844. punctata Linn.

5S45. luce Lec.

584ti. lugubris $L c c$.

Plusiotis Burm.

75847. gloriosa Lec.

5848. Leeontei Horn.

5849. Woodii Horn.

Cotalpa Burm.

5850. consobrina Horn.

7581 . lanigera Linn.

5852. flavida Horn.

5853. ursina Horn.

555t, puncticollis Lec.

5855. granicollis Hald.

Rutela Lat.

5856. formosa Burm.

Polymœchus Lec.

5557. brevipes Lec.

Cyclocephala Lat.

5858. irnmaculata Oliv.

5859. longula Lec.

5860. seritiosa Lec.

5861. nigricollis Burm.

5862. villosa Burm.

5863. hirta Lec.

5864. puberula Lcc.

5S65. dimidiata Bựm.

5866. manea Lec.

Chalepus MacL.

Dyscinetus Har.

5867. obsoletus Lec.

$\triangleleft$ 5868. trachypygus Burm.

Ligyrus Burma.

C5869. gibbosus $\mathrm{DeG}$. 
5870. ruginasus Lec.

5871. relictus Say.

b872. rugiceps Lec.

\section{Aphonus Lec.}

5873. pyriformis Lec.

f) 5874. tridentatus Say. frater Lec.

5875. hydropicus Lec.

5876. castaneus Melsh. 5877. clunalis Lec.

\section{Orizabus Fairm.}

587. Snowi Horn.

5879. ligyroides Horn.

\section{Xyloryctes Hope.}

5880. satyrus Fab.

\section{Strategus Hope.}

5981. antrus Fab. 5882. mormon Burm.

y 5883. julianus $B u r m$.

5884. Eplendens Beauv.

5885. cessus Lec.

Dynastes Kirby.

$>$ 5SS6. tityus Linn.

5587. Grantii Horn.

\section{Megasoma Kirby.}

5888. thersites Lec.

\section{Phileurus Lat.}

5889. truncatus Becuv.

5890. valgus $F a b$.

5891. illatus Iec.

5892. cribrosus Lec.

\section{Allorhina Burm.}

5893. mutabilis Gory. 5894. nitida Linn.

Gymnetis MacL.

5895. Sallei Schaum

5896. cretacea Lec.
Euphoria Burm.

5897. areata $F a b$.

5898. verticalis Horn.

5899. æstuosa Horn.

5900. discicollis Thom.

5901. Kernii Hald.

5902. hirtipes Horn.

5903. devulsa Horn.

5904. sepulchralis $F a b$.

5905. subtomentosa Mann.

5906. melancholica Gory.

5907. leucographa Gory.

5908. fascifera Lec.

5y09. fulgida $F a b$.

5910. herbacea Oliv.

5911. inda Linn.

5912. Schottii Lec.

\section{Cremastochilus Knoch.}

5913. planatus Lec.

5!14. saucius Lec.

5915. spinifer Horn.

5916. Wheeleri Lec.

5917. planipes Horn.

5918. lencostictus Burm.

5919. ineptus Horn.

5920. Schaumii Lec.

5921. Westwoodi Horn.

5922. angularis Lec.

5923. pilosicollis Horn.

5924. crinitus Lec.

5925. Knochii Lec.

5926. nitens Lec.

5927. variolosus $K i r b y$.

5928. squamulosus Lec.

5929. canaliculatus Kirby.

5930. retractus Lec.

C 5931. castaner $K$ noch.

5932. Harrisii Kirby.

\section{Osmoderma Lep.}

5933. eremicola $K$ noch.

5934. scabra Beauv.

5935. socialis Horn.

\section{Gnorimus Lep.}

5936. maculosus Knoch. 
Trichius Fab.

5937. piger $F a b$.

5938. texanus Horn.

5939. affinis Gory.

5940. bibens $F a b$.

5941. viridulus $F a b$.

5942. delta Forst.
Valgus Seriba.

5943. canaliculatus $F a b$.

5944. squamiger Beauv.

5945. californicus Horn.

SPON DYLIDE.

\section{Parandra Lat.}

5946. brunnea Fab.

5947. polita Say.

Spondylis Fab.

5948. upiformis Mann.
Scaphinus Lec.

5949. sphæricollis Lec.

\section{CERAMIB YCID $A$.}

Ergates Serv.

5950. spiculatus Lec.

Stenodontes Serv.

5951. mandibularis Fab.

\section{Mallodon Serv.}

5952. mandibularis Harold. dentiger Lec.

5953. dasystomus Say.

5954. melanopus Linn.

5955. serrulatus Lec.

Derobrachus Serv.

5956. brevicollis Serv.

5957. geminatus Lec.

Orthosoma Serv.

5958. brunneum Forst.

\section{Prionus Geoff.}

5959. Iaticollis Drury.

5960 . pocularis Dalm.

5961. califormicus Mots.

5961 l. curvatus Lec.

5962. imbricornis Linn.

5963. fissicornis Hald.

5964 . palpalis Say.
Homæsthesis Lec.

5965. integer Lec.

5966. emarginatus Say.

Tragosoma Serv.

5967. Harrisii Lec.

Sphenostethus Hald.

5968. Taslei Buq.

Elateropsis Chev.

5969. fuliginosus $F a b$.

Asemum Esch.

5970. atrum Esch.

5971. mostum Hald.

5971 a. substriatum Hald.

59716. juvencum Hald.

5972. nitidum Lec.

Nothorhina Redt.

5973. aspera Lec.

Criocephalus Muls.

5974. productus Lec.

5975. agrestis Kirby.

5976. asperatus Lec.

5977. montanus Lec. 
5978. obsoletus Rand.

5979. nubilus Lec.

5980. australis Lec.

\section{Tetropium Kirby.}

5981. velutinum Lec.

5982. cinnamopterum Kirby.

\section{Opsimus Thom.}

5983. quadrilineatus Mann.

\section{Dicentrus Lec.}

5984. Blüthneri Lec.

\section{Smodicum Hald.}

5985. cucujiforme Say.

\section{Gonocallus Lec.}

5986. collaris Kirby.

\section{Physocnemum Hald.}

Dularius Thom.

5987. Andreæ Hald.

5988. brevilineum Say.

\section{Rhopalopus Muls.}

5989. sanguinicollis Horn.

\section{Hylotrupes Serv.}

5990. bajulus Linn.

5991. amethystinus Lec.

5992. ligneus $\mathrm{Fab}$.

\section{Phymatodes Muls.}

$>$ 5993. variabilis $F a b$.

5994. infuscatus Lec.

5995. thoracicus Muls.

5996. amonus Say.

5997. blandus Lec.

5998. maculicollis Lec.

5999. obscurus Lec.

6000. ater Lec.

6001. æneus Lec.

6002. dimidiatus Kirby.

6003. vulneratus $L e c$.

j6004. varius $F a b$.

6005. decussatus Lec.
6006. nitidus Lec.

nitens err. typ.

Merium Kirby.

6007. proteus Kirby.

Callidium Fab.

6008. antennatum Newm.

6009. janthinum Lec.

6010. cicatricosum Mann.

6011. æreum Newm.

6012. hirtellum Lec.

6013. vile Lec.

\section{Xylocrius Lec.}

6014. Agassizii Lec.

6015. cribratus Lec.

Malacopterus Serv.

Ganimus Lec.

6016. vittatus $L e c$.

Oeme Newm.

6017. rigida Say.

6018. costata Lec.

6019. strangulata Horn.

6020. gracilis Lee.

Eucrossus Lec.

6021. villicornis $L e c$.

Dryobius Lee.

6022. sexfasciatus Say.

Haplidus Lec.

6023. testaceus Lec.

Achryson Serv.

6024. surinamum Linn.

6025. concolor Lec.

Hypexilis Horn.

6026. pallida Horn.

Gracilia Serv.

6027. minuta Fab.

6028. obliquata Horn. 
6029. manca Lec.

6030. fasciata Lec.

\section{Hammaticherus Serv.}

10031. mexicanus Thom.

\section{Arestinus Lec.}

6032. obscurus Lec.

\section{Brothylus Lec.}

6033. gemmulatus Lcc. 6034. conspersus Lec.

\section{Stromatium Serv.}

6035. pubescens Hald.

Osmidus Lee.

6036. guttatus Lec.

Gnaphalodes Thom.

$>$ 6037. trachyderoides Thom.

Chion Newm.

6038. cinctus Drury.

6038a. garganieus Fab.

Eburia Serv.

6039. Ulkei Bland.
6040. stigmatica Chev.
perforata Lec.
6041. Inldemani Lec.
6042. quadrigeminata Say.
6043. stigma Oliv.
6044. distincta Hald.
6045. ovicollis Lec.
6046. tumida Lec.
6047. mutica Lec.
6048 . manca Lec.

\section{Romaleum, White.}

6049. simplicicolle Hald. procerum Lcc.

6050. atomarium Drury.

6051. rufulum Hald.

[052. seminitidum Horn.

6053. treniatum Lcc.
Elaphidion Serv.

6054. irroratum Fab.

6055. alienum Lec.

6056. mueronatum Fab.

6057. tectum Lcc.

6058. incertum Nevom.

6059. inerme Ncum.

Gofo. truncatum Hald.

fi061. spureum Lec.

Y 6062. villosum Fitb.

Q 6063. parallelum Newm. aretum Neam.

6064. pumilum Ncwm.

6065. subpubescens Lcc.

6066. aculeatum Lec.

6ofit. tenue Lec.

60)(is. volitans Lec.

6069. unicolor Rand.

6070. punctatum Lec.

j071. imbelle Lec.

6072. cimerascens Lec.

6073. mostum Lec.

\section{Aneflus Lee.}

6074. ealvatus Horn.

6075. prolixus Lcc.

6076. protensus Lec.

(i077. linearis Lec.

\section{Eustroma Lec.}

6078. validum Lec.

Tylonotus Hald.

(5079. bimaculatus Hald.

Zamodes Lec.

(i080. obscurus Lec.

\section{Compsa Perty.}

6081. puncticollis Lec. 130.52. quadriplagiata Lee.

\section{Heterachthes Newm.}

(i0)3. nobilis $L(C C$.

1i084. quadrimaculatus Nevm.

6085. ebenus Newm. 
Curius Newm.

6086. dentatus Newm.

Plectromerus Lec.

6087. dentipes Oliv. scambus Newm.

Pœecilobrium Horn. Callimus $\ddagger$ Lec.

6088. chalybæum Lec.

\section{Eumichthus Lec.}

6089. œdipus Lec.

\section{Phyton Newm.}

6090. pallidum Say.

6091. discoideum Lec.

Obrium Serv.

6092. rubrum Newm.

6093. rubidum Lec.

Hybodera Lec.

6094. tuberculata Lec.

6095. debilis Lec.

Callimus Muls.

Pilema Lec.

6096. cyanipennis Lec.

6097. ruficollis Lec.

Megobrium Lec.

6098. Edwardsii Lec.

Molorchus Fab.

6099. longicollis Lec.

6100. bimaculatus Say.

- $100 a$. corni Hald.

6100b. semiustus Newm.

Callimoxys Kraatz.

6101. sanguinicollis Oliv.

(i102. fuscipennis Lec.

\section{Rhopalophora Serv.}

6103. lævicollis Lec.
6104. rugicollis Lec.

6105. longipes Say.

Ancylocera Serv.

6106. bicolor Oliv.

Elytroleptus Dugès.

Pteroplatus Buq.

6107. floridanus Lec.

6108. divisus Lec.

6109. rufipennis Lec.

6110. apicalis Lec.

6111. ignitus Lec.

Holopleura Lec.

6112. Helena Lec. marginata Lec.

\section{Rosalia Serv.}

6113. funebris Mots.

\section{Callichroma Lat.}

6114. splendidum Lec.

6115. plicatum Lec.

6116. cobaltinum Lec.

6117. melancholicum Chev.

Megaderus Serv.

6118. bifasciatus Dup.

Dendrobias Serv.

6119. quadrimaculatus Dup.

Lissonotus Dalm.

6120. multifasciatus Dup.

Stenaspis Serv.

6121. verticalis Serv.

6122. solitaria Say.

Crioprosopus Serv.

6123. splendens Lec. rimosus $\ddagger$ Lec.

6124. magnificus Ler.

0125. lateralis Lec. 
Tragidion Serv.

6126. annulatum Lec. 6127. coquus Linn. 6127a. fulvipenne Say. 6128. armatum Lec.

\section{Purpuricenus Serv.}

Y 6129. humeralis Fab. 6129a. axillaris Hald. 6130. dimidiatus Lec.

\section{Metaleptus Bates.}

6131. angulatus Chev. 6132. Batesi Horn.

\section{Aethecerus Chev.}

6133. Wilsoni Horn.

6134. Hornii Lac.

6135. latecinctus Horn.

\section{Mannophorus Lec.}

(i136. lretus Lec.

\section{Entomosterna Chev.}

6137. cyanicollis Dup.

\section{Amannus Jec.}

6138. vittiger Lec.

6139. pectoralis Lec.

Batyle 'Thom.

6i140. ignicollis Say.

6141. suturalis Say.

Oxoplus Lec.

6142. marginatus Lec.

6ilin. cruentus Lec.

6144. corallinus Lec.

6145. jocosus Horn.

Schizax Lec.

6146. senex Lec.

\section{Tylosis Lec.}

6147. maculata Lec.

6148. oculata Lec.
Crossidius Lee.

6149. ater Lec.

(315i). punctatus Lec.

6151. testacens Lec.

6152. intermedius Ulke.

6153. pulchellus Lec.

(i154. hirtipes $L c c$.

ii155. humeralis Lec.

(i156. Allegewahri Lec.

6157. discoideus Say.

Sphænothecus Dup.

6158. suturalis Lece.

Perarthrus Lee.

6159. vittatus Lec.

Ischnocnemis Thom.

6160. bivittatus Dup.

Stenosphenus Hald.

fili1. notatus Olin.

(i162. Jugens Lec.

(il63. novatus Horn. cribripennis \pm Lec.

6164. lepidus $I 1 o r n$.

6165. dolosus Horn.

6166. debilis Horn.

Trichoxys Chev.

6167. Hartwegii Whitc.

Cyllene Newm.

6168. antennatus White.

6169. crinicornis Chev.

6170. pietus Drury.

6171. robinie Forst.

6172. decorus Oliv.

Plagionotus Muls. Glycobius Lec.

6173. speciosus Say.

Calloides Lec.

y 6174. nobilis Sny. 6175. Lorquinii Buq. 
Arhopalus Serv.

Y 6176 . fulminans $F a b$.

Clytus Laich.

6177. marginicollis Lap.

6178. lanifer Lce.

Xylotrechus Chev.

6179. colonus Fab.

6180. sagittatus Germ.

6181. quadrimaculatus Fald.

6182. mormonus Lec.

618:3. undulatus S'ay.

6183a. lunulatus Kirby.

6183b. interruptus Lup.

(i183c. fuscus $\overline{\text { I }}$ rby.

6184. annosus Say.

6185. nauticus Mann.

10185a. gramineus Hald.

6186. convergens $\mathrm{Lec}$.

6187. nitidus Horn.

6188. insignis Lec.

6189. planifrons Lec.

(il90. obliteratus Lec.

\section{Neoclytus Thom.}

6191. suturalis Otiv.

6192. angulatus $F a b$.

Hopei Lap.

rhombifer Oliv.

6193. devastator Lap.

6194. irroratus Lec.

morosus Chev.

6195. seutellaris Oliv.

6196. luseus Fab.

(j197. conjunctus Lec.

6198. caprea Say.

6199. muricatulus Kirby.

6200. ascendens Lec.

6201. erythrocephalus Fab.

6202. torquatis Lee.

6203. approximatus Lec.

6204. longipes Kirby.

6205. balteatus Lec.

6206. interruptus $L c c$.

\section{Clytanthus Thom.}

6207. ruricola Oliv. 6208. albofasciatus Lap.
Microclytus Lec.

6209. gazellula Hald. gibbulus Lec.

\section{Cyrtophorus Lec.}

6210. verrucosus Oliv.

Tillomorpha Blanch.

6211. geminata Hald.

Euderces Lec.

Y 6212. picipes $F a b$.

6213. pini Oliv.

6214. parallelus Lec.

6215. Reichei Lec.

Agallissus Dalm.

6216. gratus Lec.

Zagymnus Lec.

yoit. clerinus Lec.

Atimia Hald.

6218. confusa Say.

6219. dorsalis Lec.

Distenia Serv.

6220. undata Oliv.

Desmocerus Serv.

\& 6221. palliatus Forst.

7 6222. auripennis Chev.

6223. cribripennis Horn.

6224. californicus Horn.

Necydalis Limn.

ti225. mellitus Say.

6226. lrvicollis Lec.

6227. cavipennis Ler

Ulochretes Lec.

6228. leoninus Lec.

Pyrotrichus Lec.

6229. vitticollis $L e c$. 
Leptalia Lec.

6230. macilenta Mann.

\section{Encyclops Newm.}

6231. cæruleus Say.

\section{Rhagium Fab.}

yे 6232. lineatum Oliv.

Centrodera Lec.

6233. decolorata Harr.

6234. picta Hald.

6235. sublineata Lec.

6236. nevadica Lec.

Xylosteus Frivald.

6237. ornatus Lec.

Toxotus Serv.

5238. Schaumii Lec.

6239. flavolineatus Lec.

6240. vittiger Rand.

6241. virgatus Lec.

6243. cylindricollis Say.

6244. vestitus Hald.

6245. cinnamopterus Rand.

6246. obtusus Lec.

6242 imbifir lice

Pachyta Serv.

6247. monticola Rand.

6248. liturata Kirby.

6249. armata Lec.

6250. rugipennis Newm.

6251. spurea Lec.

\section{Anthophilax Lec.}

6252. viridis Lec.

6253. malachiticus Hald.

6254. attenuatus Hald.

6255. mirificus Bland.

6256. tenebrosus Lec.

\section{Piodes Lec.}

6257. coriacea Lec.

\section{Acmæops Lec.}

6258. thoracica Hald.
6259. - bivittata Say.

6) 62601. atra Lec.

6261. subrenea Lec.

6262. pinguis Lec.

6263. tumida Lec.

6264. viola $L e c$.

6265. militaris Lec.

6266. subpilosa Lec.

6267. longicornis Kirby.

6268. vineta $L e c$.

6269. ligata Lec.

6270. basalis Lec.

6271. directa Newm.

6272. discoiden Hald.

6273. proteus Kirby.

6273a. gibbula Lec.

6274. pratensis Laich.

6275. falsa Lec.

\section{Gaurotes Lec.}

7 6276. cyanipennis Say.

A 6277. abdominalis Bland.

6278. Cressoni Bland.

\section{Bellamira Lec.}

6279. scalaris Say.

Strangalia Serv.

6280. virilis $L e c$.

6281. famelica Newm.

6282. acuminata Oliv.

6283. strigosa Newm.

Y 6254. luteicornis Fab.

6285. bicolor Swed.

6286. sexnotata Hald.

6287. delicata Lec.

Typocerus Lec.

6288. badius Newm.

6289. zebratus $F a b$.

6290. sparsus Lec.

6291. lunatus Fab.

/ 6292. velutinus Oliv.

6293. lugubris Say.

6294. brunnicornis Lec.

6295. balteatus Horn.

6296. sinuatus Nerom. 
Leptura Serv.

6297. emarginata $F a b$.

6298. gigas Lec.

6299. obliterata Hald.

6300. soror Lec.

6301. propinqua Bland.

6302. deleta Lec.

6303. plebeja Rand.

1304. subhamata Rand.

6305. abdominalis Huld.

6306. plagifera Lec. anthracina Lec.

6307. amabilıs Lec.

6308. lineola Say.

6309. rubira Lec.

6310. eruentata Hald.

6311. chalybra Hald.

6311a. cyanella Lec.

6312. capitata Newm.

6313. americana Hald.

6314. hæmatites Newm.

6315. exigua Newm.

nana Newm.

saucia Lec.

6316. subargentata Kirby.

rhodopus Lec.

ruficeps Lec.

similis Kirby.

6317. molybdica Lec.

6318. Ireta Lec.

Q 6319. zebra Oliv.

6320. tribalteata Lec.

6321. impura Lec.

6322. cordifera Oliv.

6323. instabilis Hald.

$6323 \alpha$. convexa Lec.

6324. sexmaculata Linn.

6325. quadrata Lec.

6326. sexspilota Lec.

6327. Matthewsii Lec.

6328. grossa Lec.

6329. brevicornis Lec.

6330. nigrella Say.

6331. carbonata Lec.

6332. canadensis Fab.

6232a. erythroptera Kirby.

6232b. cribripennis Lec.

6333. rubrica Say.

6334. circumiata Oliv.
6335. vagans Oliv.

6336. dehiscens Lec.

6337. sanguinea Lec.

6338. lætifica Lec.

6339. hirtella Lec.

6340. quadrillum Lec.

6341. chrysocoma Kirby.

6342. nigrolineata Bland.

6343. rufula $H$ cidd.

\&6314. proxima Say. atrata Lec.

6345. biforis Newm.

6346. dolorosa Lec.

6347. crassicornis Lec.

6348. crassipes Lec.

6349. tibialis Lec.

6350. Behrensii Lec.

6351. octonotata Say.

6352. pedalis Lec.

6353. vittata Germ.

6354. pubera Say.

6355. ruficollis Say.

$6355 \alpha$. sphæricollis Say.

7. 6356. vibex Newn.

6357. aurata Horn.

6358. scripta Lec.

> 6359. gnathoides Lec.

6360. valida $L e c$

6361. mutabilis Newm.

6362. quadricollis Lec.

6363. aspera Lec.

6364. eubitalis Iec.

6365. spuria Lec.

\section{Euryptera Serv.}

6366. lateralis Otiv.

Plectrura Mann.

6367. spinieauda Mann.

\section{Ipochus Lec.}

6368. fasciatus Lec.

Monilema Say.

6369. annulatum Say.

$>6370$. appressum Lec.

6371. gigas Lec.

6372. semipunctatum Lec.

6373. lævigatum Bland. 
6374. armatum Lcc.

6375. obtusum Lec.

6370. spoliatum Horn.

(i.377. forte Lcc.

6.378. subrugosum Bland.

6:379. Ulkei Horn.

6380. variolare Thom. albopictum $\neq$ Lec.

6381. crissum Lec.

\section{Michthysoma Lec.}

6382. heteroioxum Lec.

\section{Cyrtinus Lec.}

6383. pygmaus Hald.

\section{Psenocerus Lec.}

6384. supernotatus Say.

Monohammus Serv.

6385. titillator $F u b$. earolinensis Oliv. minor Lec.

6.386. maculosus Huld. clamator Lec.

6387. scutellatus Say. oregonenxis Ler:

> 6388. confusor Kirby. 6384. marmorator Iivby.

Ptychodes Serv.

6390. vittatus $F(b$.

\section{Dorcaschema Lec.}

6:391. Wildii Uhler.

6392. alternatum Say.

6393. nigrum Say.

\section{Hetoemis Hald.}

(i:)!4. cineren Olw.

\section{Cacoplia Lec.}

6395. jullata Hald.

Goes Lee.

0396. tigrina $D e G$.

(6):17. pulchra Hald.
6398. debilis Ler.

(i:399. tesselata $\mathrm{Hald}$

fifou. pulverulenta $H a l d$.

6401. oculati Lec.

Plectrodera Lee.

6.402. sealator Fub.

\section{Synaphœta Thom.}

6403. Guexi Lec.

\section{Acanthoderes Serv.}

6404. quadrigibbus Say.

6405. peninsularis Horn.

6406. decipiens Hald.

6107. Morrisii Uhler.

\section{Lagochirus Er.}

(i408. araneiformis $\operatorname{Lin} n$. if409. obsoletus Thom.

\section{Cœnopœus Horn.}

6it10. Palmeri Lec.

Leptostylus Lec.

6411. aculiferus Say.

6412. argentatus Duval.

(i413. terreenlor Horn.

6414. planidorsus Lec.

6.415. nebulosus Horn.

6itlf. areuatus Ler.

15+17. parvus Lec.

(itls, biustus Lec:

6419. albidus Lec.

fit20. eommixtus Hald.

6+21. eollaris Hali.

17+22. perplexus Hald.

6.12:. macula Stuy.

Liopus Serv.

(i42). variegatus $\mathrm{Hald}$.

6.424a. obscurus Hald.

(i424b. trifasciatus Hald.

1i425. Wiltii Horn.

li426. crassulus Lec.

it27. rentralis Lcc.

6.428. fascienharis Harr. xanthoxyli Shimer. 
(i429. alpha Say.

6430. cinereus Lcc.

b431. punctatus Ler.

i.432. Haldemani Lec.

Dectes Lec.

643:3. spinosus Sray.

Mecotetartus Bates.

b434. antenuatus Bates.

\section{Lepturges Bates.}

6435. symmetricus Hald. 6435a. angulatus Lec.

64356. pictus Lec.

6436. signatus Lec.

6437. querci Fitch.

6438. fucetus Say.

6439. regularis Lec.

\section{Hyperplatys Bates.}

6440. aspersus Say.

6441. maculatus $H a l d$.

64tIr, nigrellus Hald.

6441b. femoralis Hald.

\section{Urographis Horn.}

6412. triangulifer Huld. 644:\% fasciatus $D e G$.

Graphisurus İirby.

6444. pusillus Kirby.

Acanthocinus Steph.

6445. obsoletus Oliv.

6446. obliquus Lec.

6447. spectabilis Lec.

6448. nodosus Fab.

Estola Fairm.

6449. sordida Lee.

Hoplosia Muls.

6450. nubila Lec.

Pogonocherus Lat.

6451. crinitus Lec.
6452. penicellatus Lec.

(5)453. mixtus Hrid.

6451. oregonus Lec.

6455. volitans Lec.

Ecyrus Lec.

6.456. dasycerus Say.

6457. exiguus Lec.

Eupogonius Lec.

6458. tomentosus Hald.

6459. vestitus Say. pauper Lec.

6460. pubeseens Lec.

6461. subarmatus Lec.

Lypsimena Lec.

14462. fuscata Lec.

6463. culifornica Horm.

Zaplous Lec.

6464. Hubbardi Lec.

Desmiphora Serv.

6465. mexicana Thom.

Oncideres Serv.

6466. pustulata Lec.

6467. putator Thom.

6468. texana Horn.

6469. cingulata Say.

Taricanus Thom.

6470. Truquii Thom.

Ataxia Hald.

6471. crypta $S a y$.

Hippopsis Serv.

6472. lemniscata $F a b$.

Dorcasta Pasc.

6473. cinerea Horn.

Sicyobius Horn.

6474. Brousii Horn. 
Spalacopsis Newm.

6475. stolata Newm.

6476. suffusa Newm.

\section{Saperda Fab.}

6477. obliqua Say.

6 6478. calcarata Say.

6478a. adspersa Lec.

5 6479. mutica Say.

Y 6480. candida Fab.

6481. cretata Newm.

6482. Fayi Bland.

6483. vestita Say.

6484. discoidea Fab.

6185. tridentata Oliv.

6486. lateralis $F a b$.

6487. puncticollis Say.

6488. mosta Lec.

A 6489. concolor Lec.

Mecas Lec.

6490. inornata Say.

6491. marginella Lec.

6492. pergrata Say.

6493. femoralis Hald.

6494. ruficollis Horn.

\section{Oberea Muls.}

6495. oculaticollis Say.

6496. bimaculata Oliv. perspicillata Hald.

6496a. tripunctata \| Fab.

6496b. basalis Lec.

fi497. quadricallosa Lec.

6498. Schaumii Lec.

6499. tibialis Hald.

6500. Alavipes Hald.

i501. ocellata Hreld.

6501a. discoidea Lec.

6502. texana Horn.

6503. tripunctata Swed. amabilis Hald. 6503a. myops Hald.

6503b. mandarina Fab.

6504. gracilis Fab.

6505. ruficollis $\mathrm{Fab}$.

Tetrops Steph.

6506. canescens Lec.

6507. jucunda Lec.

6508. monostigma Hald.

Tetraopes Serv.

6509. discoideus Lec.

6510. canteriator Drap.

6511. tetraophthalmus Forst.

6512. collaris Horn.

4 6513. femoratus Lec.

6513a. mancus Lec.

6513b. basalis Lec.

6513c. oregonensis Lec.

6514. quinquemaculatus Hald.

6514a. texanus Horn.

6515. canescens Lec.

\section{Amphionycha Lec.}

6516. flammata Newm.

Idœmea Horn.

6517. Fulleri Horn.

Styloxus Lec.

6518. Iucanus Lec.

Methia Newm.

Thin Newm.

6519. pusilla Nerom.

Dysphaga Lec.

6520. tenuipes Hald.

6521. Lævis Lec.

6522. bicolor Horn.

CHIR YSOUEIIDA.

Donacia Fab.

6523. pubicollis Suffr.

6524. Harrisii Lec.

6525. rugifrons Newm. lucida Lac.
\& 6526. palmata Oliv.

6527. hypoleuca Lac.

6528. texana $\mathrm{Cr}$.

76529. piscatrix Lac.

6530. tuberculata Lac. 
6531. porosicoilis tac.

6532. hirticollis Kirby.

6433. maynifica Lec.

6434. proxima Kirby.

f4435. distincta $L c e$.

6436. subtilis I Iunze.

1437. rugosa Lec.

155 3338. pubescens Lcc.

(i) H39. requalis Say. confusa Lec.

65 A440. torosa Lec.

65 A41. elnarginata Kirby.

65 A42. pyritosa Lec.

(15) A13. cuprea Kirby. cataractre Nerom.

(i5 A4t. femoralis Kirby. flavipennis Mann.

105 fi45. metallica $A h r$.

65 H46. flavipes Kirby.

li5 6447. jucunda Lec.

Bis f448. Kirbyi Lac.

- rea Lac.

- assimilis Lac.

- binodosa Germ,

- earolina Lac.

-_. chalcea Lac.

-_- crerulea Oliv.

- dentata Fope. Hoppe

- fulvipes Lac.

-- militaris Lac.

-_ nitida Germ.

-_ pallipes Lac.

-— parva Lac.

-_ rufa Say.

- vicina $L a c$.

\section{Hæmonia Lat.}

6549. nigricornis Kirby.

\section{Orsodachna Lat.}

7 6550. atra $A h r$. Childreni Kirby.

luctuosa Lac.

tricolor Melsh.

\section{Zeugophora Kunze.}

6551. scutellaris Suffr.

6552. abnormis Lec;

6553. puberula $C r$.
6554. varians $\mathrm{Cr}$.

Reineckii Grote.

6555. californica $\mathrm{Cr}$.

6556. consanguinea $\mathrm{Cr}$.

__ Kirbyi Baly.

Syneta Esch.

6557. ferruginea Germ.

6558. carinata Mfann.

6559. albida Lec.

6560. simplex Lec.

Thricolema Cr.

6561. anumala $\mathrm{Cr}$.

\section{Lema Fab.}

6562. cornuta $F a b$.

6563. concolor Lec.

6564. texana $\mathrm{Cr}$.

6565. brunnicollis Lac.

6566. maculicollis Lac.

6567. collaris Say.

6568. peninsulæ $C r$.

6569. solani Fab.

6570. circumvittata Clark.

6571. balteata Lec.

6572. conjuncta Lac.

7 6573. trilineata Oliv.

6574. nigrovittata Guér.

6575. 6-punctata Oliv.

$76575 a$. ephippiata Lac.

6575b. albini Lac.

6576. Sayi $\mathrm{Cr}$.

- melanocephala Say.

_- pubipes Clark.

Crioceris Geoff.

6577. asparagi $\operatorname{Linn}$.

6578. 12-punctatus Linn.

\section{Anomøa Lac.}

6579. laticlavia Forst.

6580. mutabilis Lac.

6581. militaris Lec.

\section{Euryscopa Lac.}

6582. Lecontei $\mathrm{Cr}$.

6583. vittata $L e c$. 
Coscinoptera Lac.

6584. æneipennis Lec.

6585. renescens $\mathrm{Cr}$.

6586) bifaria Lec.

6587. axillaris Lec.

6588. canella Lec.

6589. mucorea Lec.

6590. dominicana Fab.

6591. dorsalis Lee.

6592. vittigera $L e c$.

\section{Megalostomis Chev.}

6593. pyropyga Lac.

6594. major $\mathrm{Cr}$.

6595. subfasciata Lec.

\section{Babia Chev.}

6596. 4-guttata Oliv.

6596a. pulla Lec.

6596ib. tetraspilota Lec.

\section{Saxinis Lae.}

6597. apiealis Lee.

6598. omogera Lac.

6599. sancia Lec.

\section{Urodera Lac.}

6600. crucifera Lac.

Chlamys Inoch.

$Y_{6601 .}$ plicata Fab.

(i60)1 $\alpha$, assimilis $K l$.

$6601 b$. polycocea $L a c$.

G601c. tubereulata $K l$.

(6602. cribripennis Lec.

fico3. foveslata Knoch.

\section{Exema Lac.}

(i604. gibber Oliv.

6605. conspersa M I Tann. 6605 . dispar Lec.

\section{Bassareus Inald.}

6606. congestus Fub.

6607. areolatus Suffr.

bitios. formosus MYelsh.

titiosa. sulfuripennis Melsh. fi609. detritus Oliv.

fiti10. mammifer Newm.

6iti10a. sellatus $S u f f r$.

(fifiob. pretiosus Melsh.

titilne. luteipennis Melsh.

bitilld. egenus Suffr.

66i11. croceipennis Lec.

6612. lituratus Fab.

fitil 2 ?. recurvus Say.

6612b. lativittis Germ.

6612c. vittatus Suffr.

5-vittatus err. typ.

Cryptocephalus Geoft:

6i613. mucorens Lec.

? basalis Suffr.

6614. t-maculatus Say.

fi61 $4 \alpha$. notatus $F a b$.

6614b. fulvipennis Hald.

6615. quadriforis Newm.

6616. binominis Newm. distinctus Hald.

6017. quadruplex Newm.

fi617a. 4-guttulus Suffr.

6618. sanguinicollis Suffr.

(6ilsa. nigerrimus $\mathrm{Cr}$.

6619. guttulatus Oliv.

6ii20. cribripennis Lec.

6621. bivius Newm.

(iti22. lencomelus Suffr.

6iti22 $\imath$. vitticollis Lee.

1962:3. eastanens Lec.

6624. amatus Hald.

16i25. defectus Lec.

(i626. contluens Say.

66i27. spureus Lec.

fiti28. venustus $F u b$.

flaceidus Suffr.

li62s $x$. ornatus $F a b$.

fifizsb. hamatus Melsh.

liti2sc. cinctipennis Rand.

662\&d. simplex HIald.

fii29. obsoletus Germ.

titi30. nanus $F a b$.

(iti31. cirinatus Lee.

66i32. insertus Hald. ellipsoidalis Casey.

6633. calidus Suffr

? bispinus Suffr.

bifi34. albieans Hald. 
1635. gibbicollis Hald.

6636. auliens Hald.

6637. trivittatus Oliv.

6038. incertus Oliv.

6639. pumilus Hald.

6640. mutabilis Melsh. dispersus Hald.

6641. fulguratus Lec.

6642. badius Suffr.

6643. Schreibersii Suffi。

66i44. tinctus Lec.

6645. lateritius Newm.

6646. striatulus Lec.

66.47. luteolus Nevm.

__ pseudolis Suffr.

luscus Suffr.

punctipes Suffr.

4-signatus Suffr.

\section{Griburius Hald.}

6648. larvatus Newm.

6649. scutellaris $F a b$.

6650. equestris Oliv.

6651. Montezumæ Suffr.

6if52. Luecontei $\mathrm{Cr}$.

\section{Pachybrachys Chev.}

6653. Xanti $\mathrm{Cr}$.

6654. Donneri Cr.

6655. morosus Hald.

6656. analis Lec.

6657. striatus Lec.

6658. virgatus Lec.

6659. litigiosus Suffr.

6660. abdominalis Say.

n661. dubiosus Lec.

6662. othonus Say.

6663. pallidipenn is Suffr.

6604. eireumeinetus $\mathrm{Cr}$.

6665. viduatus $\mathrm{Fab}$.

albescens Suffr.

6666. picturatus Germ.

M-nigrum Melsh.

b667. pulvinatus Suffr.

6668. limbatus Nerom.

6669. eruentus Lec.

6670. hybridus Suffr.

6671. trinotatus Melsh.

6672. intricatus Suffr.
6673. tridens Melsh.

6674. impurus Suffr.

6675. sobrinus Hald.

6676. celatus Lec.

6677. melanostictus Suffr.

6678. livens Lec.

6679. peccans Suffr.

6680. xanthias Suffr.

6i61. obsoletus Suffr.

6682. conformis Suffr.

6683. carbonarius Hald.

6684. nigrieornis Say.

6685. lustrans Lec.

6686. renidens Lec.

6ib87. subvittatus Lec.

16688. turbidus Lec.

6i689. luridus $F a b$.

16690. atomarius Melsh.

(i691. femoratus Olvv.

(i69)2. infaustus Hold.

(i69)3. characteristicus Suffr.

16994. oculatus Suttr.

6695. spumarius Suffir.

6696. brevicollis Lec.

6697. hepaticus Melsh.

6i698. subfasciatus Hald.

6699. signatifrons MTann.

6700. luctuosus Suffr.

6701. dilatatus Suffr.

-_- flavicornis Melsh.

- modestus Fab.

--_ pectoralis Melsh.

-

\section{Monachus Chev.}

6702. ater Hald.

6703. saponatus Fub.

6704. thoracicus $\mathrm{C}^{r}$.

6705. auritus Hald.

6705a. affinis Hald.

6706. Guerini Perb.

-_._ seminulum Suffr.

\section{Diachus Lec.}

6707. auratus Fab.

6708. erasus Lec.

6709. levis Hald.

6710. squalens Suffr.

6711. æruginosus Lec. 
6712. catarius Suffr.

6713. pallidicornis Suffr.

6714. chlorizans Suffr.

\section{Triachus Lec.}

6715. atomus Suffr?

6716. cerinus Lec.

6717. vacuus Lec.

6718. postremus Lec.

\section{Trichotheca Baly.}

6719. vagans Lec.

Xanthonia Baly.

6720. 10-notata Say.

6721. villosula Melsh.

6722. Stevensii Baly.

\section{Fidia Baly.}

6723. murina $C_{r}$.

6724. longipes Melsh.

7 6i2.). viticida Walsh

\section{Adoxus Kirby.}

6726. vitis Linn.

\section{Glyptoscelis Lec.}

6727. pubescens Fab.

6728. illustris $\mathrm{Cr}$.

67729. albidus Lec.

6730. barbatus Say.

6731. squamulatus $\mathrm{Cr}$.

6732. crypticus Say.

6733. alternatus $\mathrm{Cr}$.

6734. cuprascens Lec.

6735. smaragdulus Lec.

6736. varicolor $\mathrm{Cr}$.

6737. longior Lec.

Myochrous Er.

6738. denticollis Say.

6739. longulus Lec.

6740. squamosus Lec.

-- ? plagiata Melsh.

\section{Chrysochus Chev.}

6741. auratus Fab.
6742. cobaltinus Lec.

6743. robustus Horn.

\section{Tymnes Chap.}

6744. tricolor Fab. picipes Oliv.

6745. metasternalis $\mathrm{Cr}$.

6746. oregonensis $\mathrm{Cr}$.

\section{Paria Lec.}

liit7. 6-notata Say. 6747a. 4-guttata Lec. 67+76. 4-notata Say. 6748. aterrima Oliv. 6748a. opacicollis Lec. 6749. lavicollis $\mathrm{Cr}$.

6750. pumila Lec.

6751. viridicyanea $\mathrm{Cr}$.

- thoracica Melsh.

- melanura Melsh.

- canella Fab.

\section{Metachroma Lec.}

6752. maculipennis $S z$.

6753, angustula $\mathrm{Cr}$.

(i754. californica $\mathrm{Cr}$.

6755. usta Lec.

6756. dubiosa Say.

6757. interrupta Say.

6758. peninsularis $\mathrm{Cr}$.

6759. quercata Fab.

6760. vicina $\mathrm{Cr}$.

6761. marginalis $\mathrm{Cr}$.

6762. Horidana $\mathrm{Cr}$.

6763. pallida Say.

6764. levicollis $\mathrm{Cr}$.

6765. pellucida $\mathrm{Cr}$.

6766. Iateralis $\mathrm{Cr}$.

--_ atrata Fab.

- cuprea Prov.

\section{Graphops Lec.}

Q6767. pubescens Melsh.

76768. curtipennis Melsh.

6769. mareassita $\mathrm{Cr}$.

6770. simplex Lcc.

6771. nebulosus Lec.

smaragdulus Lec. 
Chrysodina Baly.

7 6772. globosa Say.

\section{Colaspis Fab.}

6773. favosa Say. 6774. brunnea Fab. 6774a. flavida Say. 6774b. costipennis $D e j$. 6775. prætexta Say. 6776. arizonæ $\mathrm{Cr}$. 6777. nigrocyanea $\mathrm{Cr}$. 6778. tristis Oliv. 6778a. ennvexa Say. 6778b. puncticollis Say.

- californica Boh. chrysis Oliv.

\section{Metaparia Cr.}

6779. clytruides $C r$,

\section{Timarcha Lat.}

6780. intricata Hald.

\section{Entomoscelis Chev.}

6781. adonidis Fab.

\section{Prasocuris Lat.}

6782. Phellandrii Linn. 6783. varipes Lec. 67S4. obliquata Lec.

\section{Doryphora III.}

6785. clivienllis Kirby. 6785a. Rogersii Lec. 6786. vittata Oliv. 6787. lineolata Stizl. 678s. 11-lineata Stäl.

8 6789. 10-lineata Say. 6789a. defecta Sticl. 6790. juncta Germ. 6791. Dahlbomi Stäl. 6792. Haldemani Rog. 6793. melanothorax Stal. 6794. rubiginosa Rog.

\section{Chrysomela Linn.}

6795. exclamationis Fab.
6796. conjuncta Rog.

6797. opifera Stäl.

>े 6798. continua Lec.

- 6799. suturalis Fab.

6800. disrupta $R o g$.

- 5 6801. similis Rog.

6802. cephalanthi $S z$.

6803. incisa $R o g$.

6804. præcelsis $R o g$.

6805. elegans Oliv.

6806. festiva $F a b$.

7 6807. lunata $F a b$.

7 6808. scalaris Lec.

- 6809. philadelphica Linn.

6809a. spirææ Say.

6810. multipunctata Say.

7 6810a. Bigsbyana Kirby.

6810b. verrucosa Suffr.

6811. dislocata Rog.

6812. sigmoidea Lec.

6813. tortuosa Rog.

6814. pnirsa Stäl.

6815. serpentina Rog.

L' 6816. multiguttata Stäl.

6817. sylvia Stäl.

6818. subsuleata Mann.

7 6819. flavomarginata Say.

6820. basilaris Say.

7 6821. auripennis Say.

6821a. cribraria Rog.

6822. montivagans Lec.

6823. inornata $R o g$.

6824. subopaca Rog. opacipennis $\mathrm{Cr}$. err. typ.

\section{Plagiodera Redt.}

6825. prasinella Lec.

6826. cochleariæ Gyll.

6827. oviformis Lec.

6828. viridis Melsh.

6829. æruginosa Suffr.

Gastroidea Hope.

$\checkmark$ 6830. polygoni Linn.

6831. dissimilis Say.

6832. cyanea Melsh

6832a. cæsia Rog.

6833. formosa Say.

6834. ænea Melsh. 


\section{Lina Meg.}

6835. californica Rog.

6836. arizonie $\mathrm{Cr}$.

6837. lapponica Linn.

68.38. tremulre $F a b$.

6839. seripta Fab.

6839a. confluens Rog.

6840. obsoleta Say.

6841. Hosculosa Stril.

\section{Gonioctena Redt.}

1842. aretien Mann.

6843. pallida Linn.

- rufipes DeG.

\section{Phyllodecta Kirby.}

6844. vulgatissima Linn.

\section{Cerotoma Chev.}

6845. caminea Fab.

\section{Andrector Horn.}

684ii. (i-punetatus Horn.

\section{Phyllobrotica Redt.}

15847. decorata Say.

6848. discoidea Fiab.

6849. viridipennis Lec.

6850. luperina Lec.

6sosl. Lurida Lec.

\section{Phyllechthrus Lec.}

6852. dorsalis Oliv. 6852a. atriventris Say.

6853. gentilis Lec.

6851. nigripennis Lec.

6855. texanus Lec.

Luperus Geoff:

6856. thoracieus Melsh.

6857. Iuteicollis Lec.

1858. fibulatus Germ.

6859. bivittatus $L e c$.

G8gut. Alavieollis Lec.

6861. varicornis Lec.

6862. smaragdinus Lec.

6863. graptoderoides $\mathrm{Cr}$.
6864. varipes Lec.

6865. cyanellus Lec.

6866. nigrocyaneus Lec.

6867. Lecontei $\mathrm{Cr}$.

6868. meraca Say.

6869. longulus Lec.

6870. morulus Lec.

6871. brunneus $\mathrm{Cr}$.

__ ? thoracicus Boh.

\section{Androlyperus $\mathrm{Cr}$.}

6872. fulvus $\mathrm{Cr}$.

6873. maculatus Lec.

\section{Scelolyperus Cr.}

6874. tejonicus $\mathrm{Cr}$.

Agelastica Redt.

6875. halensis Linn.

6876. bicolor Lec.

Metacycla Baly.

6877. insolita Lec.

Monocesta Clark.

6878. coryli Say.

Diabrotica Chev.

6879. tricincta Say.

6880. connexa Lec.

76851 . 12-punctata Oliv.

6881 a. tenella Lec.

6882. soror Lec.

6883. balteata Lec.

6884. blandula Lec.

6s85. vittata Fab.

68s6. trivittata MIann.

6857. virgifera Lec.

6888. longicornis Say.

6889. lemniseata Lec.

6890. vincta Lce.

6891. atripennis Say.

6891a. fossata Lec.

_- ? 8-notata Boh.

- ? amœnula Boh.

Trirhabda Iec.

6892. tomentosa Linn. 
6892a. virgata Lec.

6892b. canadensis Kirby.

6893. flavolimbata Mann.

? dorsata Say.

6893a. luteocincta Lec.

6894. attenuata Say.

6895. convergens Lec.

6896. nitidicollis Lec.

6896a. Lewisii $\mathrm{Cr}$.

6897. brevicollis Lec.

\section{Adimonia Leach.}

6898. externa Say.

6899. americana Fab.

6899a. cribrata Lec.

6900. conferta Lec.

6901. 6-vittata Lec.

6902. cavicollis Lec.

6903. rufosanguinea Say.

\section{Galeruca Geoff.}

Galerucella Cr.

6904. tuberculata Say.

6905. marginella Kirby.

6905a. punctipennis Mann.

8 6906. sagittarix Gyll.

6907. decora Say.

6908. carbo Lec.

6909. notulata Fab.

? puncticollis Say.

6910. notata $F a b$.

6911. integra Lec.

6912. xanthomelæena Schr.

6913. maritima Lec.

6914. morosa Lec.

6915. erosa Lec.

\section{Monoxia Lec.}

6916. guttulata Lec.

6916a. angularis Lec.

6917. consputa Lec.

6918. sordida Lec.

_- ? atomaria Fab.

\section{Blepharida Rog.}

yे 6919. rhois Forst.

Hypolampsis Clark.

6920. pilosa $I I l$.
6921. Clarkii $\mathrm{Cr}$.

6922. Mellyi $\mathrm{Cr}$.

6923. guttata Lec.

-._ rugosa. Oliv.

Phædromus Clark.

6924. paradoxus Melsh.

6925. Waterhousei Clark.

Pachyonychus Chev.

Hamletia Cr.

6926. dimidiaticornis $\mathrm{Cr}$.

\section{Oedionychis Lat.}

6927. opacior $\mathrm{Cr}$.

6928. gibbitarsis Say.

6929. lustrans $C r$.

6930. 8-maculata $\mathrm{Cr}$.

6931. interjectionis $\mathrm{Cr}$.

6932. vians $I l l$.

6932a. scripticollis Say.

6932b. discicollis Dej.

6932c. concinna Fab.

6933. lugens Lec.

6934. violascens Lec.

6935. thoraciea Fab.

6936. fimbriata Forst. suturella Say.

6937. Havocyanea $\mathrm{Cr}$.

6938. petaurista $F a b$.

7 6939. miniata Fab.

6940. longula Harold.

6941. thyamoides $\mathrm{Cr}$.

6942. texana $\mathrm{Cr}$.

6943. indigoptera Lec.

6944. 6-maculata Ill.

6945. quercata $F a b$.

6945a. suturalis $F a b$.

6945b. limbalis Melsh.

6946. scalaris Melsh.

_- jocosa Harold. obsidiana $F a b$.

Disonycha Chev.

6947. limbicollis Lec.

6947a. pallipes $\mathrm{Cr}$.

6948. alternata Ill.

6949. punctigera Lec. pulchra Casey. 
7 6950. pennsylvanica $I l l$. procera Casey.

6951. glabrata Fab.

6952. maritima Mann.

6953. arizonæ Cascy.

6954. abbreviata Melsh.

6955. discoidea Fab.

6956. funerea Rand.

6957. triangularis Say.

; 6958. collaris Fab.

6958a. collata Fab.

6958b. cervicalis Lec.

6958c. semicarbonata Lec.

6959. rufa $I l l$.

- caroliniana Fab.

\section{Haltica Genft:}

Graptodera Chev.

7 6960. bimarginata Say.

6961. chalybea Ill.

6962. carinata Germ.

6963. ignita Ill.

6964. inærata Lec.

6965. obolina Lec.

6966. obliterata Lec.

6967. torquata Lec.

6968. evicta Lec.

6969. xruginosa Lec.

6970. tincta Lec.

6971. folincer Lec.

6972. punctipennis Lec.

6973. Jazulina Lec.

6974. californica Mann.

-__ fiscorenea Melsh.

_- kalmire Melsh.

- - tombacina ITann.

verticalis Boh.

\section{Lactica Er.}

6975. ocreatia Say.

6976. specularis Harold.

\section{Cæporis Clark.}

6977. Burgessi $\mathrm{Cr}$.

6978. nana $C r$.

nanula Lec.
Crepidodera Chev.

Epitrix Foud.

6979. rufipes $\operatorname{Linn}$.

6980. Helxines Linn.

6981. atriventris Melsh.

6982. Modeeri Linn.

B982u. mancula Lec.

6983. scabricula $\mathrm{Cr}$.

6984. basalis $\mathrm{Cr}$.

6985. robusta Lec.

6986. brevis $\$ z$.

6987. cucumeris Harr.

6988. suberinita Lec.

6989. fuscula $C r$.

6990. lobata $C r$.

6991. parvula $F a b$.

__ iris Oliv.

\section{Cerataltica Cr.}

6992. insolita Melsh.

\section{Orthaltica $\mathrm{Cr}$.}

6993. copalina Fab.

6994. recticollis Lec.

\section{Systena Chev.}

6995. hudsonias Forst.

6996. frontal is $F a b$.

6997. pallipes $S z$.

6998. subenea Lec.

6999. collaris $\mathrm{Cr}$.

7000. elongata $F a b$.

7001. mitis Lec.

$7001 \%$. ligata Lec.

7001b. ochracen Lec.

7002. marginalis Ill.

7003. Dlanda Melsh.

7003a. bitæniata Lec.

_- pallidula Boh.

\section{Luperaltica Cr.}

7004. fuseula Lec.

7005. senilis Say.

\section{Longitarsus Lat.}

7006. rubidus Lec.

7007. melanurus Melsh. 
7008. testaceus foc. $\mathrm{Mg} / \mathrm{s}$

7009. repandus Lec.

7010. livens Lec.

7011. subrufus Lec.

7012. mancus Lec.

7013. ealifornicus Mots.

7014. nigripalpis Lec.

7015. alternatus Ziegl.

-.. Iituratus Oliv.

\section{Glyptina Lec.}

7016. spuria Lec.

7016 . lissotorques Lec.

7017. cerina Lec.

7018. cyanipennis $C r$.

\section{Aphthona Chev.}

7019. picta Sry.

7020. texana $\mathrm{Cr}$.

\section{Phyllotreta Foud.}

7021. lepislula $L c c$.

7022. sinuata Steph.

71)23. vittata Fub.

i024. ramosa $C$.

7025. oregonensis $C r$.

7026. bipustulata Fub.

7027. albionica Lce.

7025. Lewisii $\mathrm{Cr}$.

7029. Eneicollis $\mathrm{Cr}$.

7030. chalybeipennis $\mathrm{Cr}$.

7031. robusta Lec.

\section{Mantura Steph.}

7032. Horidana $\mathrm{Cr}$.

\section{Podagrica Chev.}

\section{Spharoderma Steph.}

7033. opima Lec.

\section{Argopistes Mots.}

7034. scyrtoides Lec.

\section{Euplectroscelis $\mathrm{Cr}$.}

7035. Xanti $\mathrm{Cr}$.

\section{Chætocnema Steph.}

7036. eribrata Lcc.

7037. rudis Lec.

7038. irregularis Lec.

7039. subcylindrica Lec. cylindrica \| Lec. hiemalis $A$ ust.

7040. denticulata IIl.

7040a. texana $\mathrm{Cr}$.

7041. pinguis Lec.

7042. protensa Lec.

7043. cribrifrons Lec.

7044. elongatula $\mathrm{Cr}$.

7045. alutacea $C_{1}$.

7046. subviridis $L c c$.

7047. decipiens $L e c$.

7048. quadricollis $S z$.

7049. crenulata $C r^{\circ}$.

7050. parcepunctata $\mathrm{Cr}$. paupercula Casey.

7051. opacula Lec.

7052. æneola Lec.

7053. pulicaria Gr. $M 7 \varepsilon / S$

7054. Alavicornis Lec.

7055. confinis $\mathrm{Cr}$.

7056. obesula $L c c$.

- americana Mots. minuta Melsh. semichalcea $M c l s h$.

Dibolia Chev.

\& 7057. ærea Alelsh.

$7057 a$. ovata Lec.

\section{Psylliodes Lat.}

7058. punctulata Melsh. ænescens Casey. extricata Casey.

7059. interstitialis Lec.

Microrhopala Baly.

7060. vittata $F a b$.

7061. dimidiata Horn.

7062. Xerene Newm.

7063. rubrolineata Mann.

7064. vulnerata Horn.

7065. floridana $\$ z$.

7066. Erebus Newm. 
7067. exeavata Oliv.

7068. cyanea Say.

7069. uniformis Smith.

7070. porcata Melsh.

7071. montana Horn.

7072. Melsheimeri $\mathrm{Cr}$.

\section{Odontota Chev.}

7073. collaris Say. Harrisi $\mathrm{Cr}$.

7074. omogera $\mathrm{Cr}$.

7075. scapularis Oliv.

7076. notata Oliv.

7077. bicolor Oliv.

7078. Horni Smith.

7079. dorsalis Thunb. sentellaris Oliv.

7080. rubra $W e b$.

7081. californica Horn.

7082. nervosa Panz. inæqualis $W e b$. rosea $W e b$.

7083. gracilis Horn.

7054. lateritia Snith.

\section{Charistena Baly.}

7085. nigrita Oliv.

7086. perspicua Horn.

7087. bicolor Smith.

7088. Ariadne Newm.

7089. Lecontei Baly.

Octotoma Suffr.

7090. plicatula Fab.

7091. marginicollis Horn.

\section{Stenopodius Horn.}

7092. flavidus Horn.
Stenispa Baly.

7093. metallica $F a b$.

7094. collaris Buly.

Porphyraspis Hope.

Y 7095. cyanea Say.

Physonota Boh.

7096. unipunctata Say. septentrionalis Boh.

Cassida Linn.

7097. nigripes Oliv.

$7097 a$. atripes Lec.

7097b. ellipsis Lec.

7098. bivittata Say.

7099. thoracica $\mathrm{Ill}$.

7100. texana $\mathrm{Cr}$.

7101. callosa Boh.

Coptocycla Chev.

A 7102. aurichalcea $F a b$. ? marylandica Hbst.

7103. arizonæ $\mathrm{Cr}$.

9 7104. guttata Oliv.

7105. purpurata Boh.

7106. Lecontei $C r$.

7107. clavata Fab.

-_ ? bisignata Boh.

- plicata Boh.

Mesomphalia Boh.

7108. Chevrolatii Boh.

Chelymorpha Chev.

7 7109. argus Licht. 17-punctata Say.

7110. phytophagica $\mathrm{Cr}$.

\section{IB IR UCHI D E.}

Spermophagus Sch.

7111. robinix Sch.

\section{Caryoborus Sch.}

7112. arthriticus Fab.

7113. Veseyi Horn.
Bruchus Iinn.

7114. rufimanus Sch.

7115. pisi Linn.

7116. mimus Say.

7117. scutellaris $F a b$.

7118. 4-maculatus $F a b$.

7119. sordidus Horn. 
7120 . biguttellus Sch.

7121. sinuatus Sch.

7122. maculatus Fab.

7123. ambiguus Sch.

7124. discoideus Say.

7125. coryphx Oliv.

7126. ramicornis Boh. cubiculus Casey.

7127. Ulkei Horn.

7128. bivulneratus Horn.

7129. cruentatus Horn.

7130. limbatus Horn.

7131. diseolor Horn.

7132. nigrinus Horn.

7133. pruininus Horn.

7134. desertorum Lec.

7135. aureolus Horn.

7136. pauperculus Lec.

7137. prosopis Lec.

7138. floridie Horn.

7139. protractus Horn.

7140. inornatus Horn.

7141. pectoralis Horn.

7142. uniformis Lee.

7143. bisignatus Horn.

7144. alboscutellatus Horn.
7145. perforatus Forn.

7146. distinguendus Horn.

7147. ealvus Horn.

7148. fraterculus Horn.

7149. amicus Horn.

7150. obsoletus Say. fabæ Riley.

7151. hibisei Oliv.

7152. longistilus Horn.

7153. Schrankire Horn.

7154. mixtus Horn.

7155. musculus Say.

7156. placidus Horn.

7157. exiguus Horn.

7158. seminulum Horn.

7159. macrocerus Horn.

7 I60. californicus $B o h$.

- atomarius Boh.

Zabrotes IIorn.

7161. cruciger Horn.

7162. spectabilis Horn.

7163. obliteratus Horn.

71fit. densus Horn.

7165. planifrons Horn.

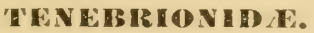

\section{Craniotus IJec.}

7166. pubescens Lcc.

\section{Edrotes Lec.}

7167. rotundus Say.

7168. ventricosus Lec.

\section{Triorophus Lec.}

7169. nodiceps Lec.

7170. punctatus Lec.

7171. lrevis Lec.

7172. subpubescens Horn.

\section{Stibia Horn.}

7173. puncticollis Horn.

7174. ovipennis Horn.

7175. hispidula Horn.

\section{Triphalus Lec.}

7176. perforatus Lec.
Trimytis Lee.

7177. pruinosa Lec.

7178. pulverea Horn.

\section{Concecus Horn.}

7179. ovipennis Horn.

Auchmobius Lec.

7180. sublrevis Lec.

\section{Eurymetopon Esch.}

7181. inflatum $L \in c$.

7182. rufipes Esch.

7183. emarginatum Casey. carbonatum Casey 오 papagonum Casey. piceum Casey. seulptile Cusey.

7184. dubium Cuscy. carbonatum Casey $\delta$. 
7185. convexicolle Lec.

7186, bicolor Horn.

7187. punctulatum Lec.

7188. solalis Horn.

7159. serratum Lec.

\section{Emmenastus Mots.}

7190. texanus Lec.

7191. longulus Lec.

7192. punctatus Lec.

7193. subopacus Horn.

7194. pinguis Lec.

7195. ater Lec.

7196. acutus Horn.

7197. obesus Lec.

7198. nanulus Casey.

7199. obtusus Lec.

__ ? rugosus Mots.

\section{Epitragus Lat.}

7200. submetallicus Lec.

C 7201. acutus Lec.

7202. arundinis Lec.

C 7203. canaliculatus Say.

7204. pruinosus Horn.

7205. dentiger Horn.

7206. tomentosus Lec.

7207. plumbeus Lec.

7208. ovalis Casey.

\section{Schøonicus Lec.}

7209. puberulus Lec.

\section{Chilometopon Horn.}

7210. abnorme Horn.

7211. helopioides Horn.

\section{Cnemodus Horn.}

7212. testaceus Horn.

Batulius Lec.

7213. setosus Lec.

7214. rotundicollis Lec.

\section{Zopherus Lap.}

7215. Haldemani Sallé.

7216. elegans Horn.

7217. concolor Lec.
7218. guttulatus Horn.

7219. tristis Lec.

7220. opacus Horn.

7221. gracilis Horn.

7222. granicollis Hor .

Phløodes Lec.

$>$ 7223. diabolicus $L \epsilon c$.

Noserus Lcc.

7224. plicatus Lec.

7225. emarginatus Horn.

\section{Phellopsis Lec.}

7226. obeordata Kirby. $7226 a$. poreata Lec.

Usechus Mots.

7227. lacerta Mots.

Dacoderus Lee.

7228. striaticeps Lec.

\section{Aræoschizus Lec.}

7229. costipennis Lec.

7230. sulcicollis Horn.

7231. regularis Horn.

7232. armatus Horn.

Anepsius Lec.

7233. delicatulus Lec.

Nyctoporis Esch.

7234. eristata Esch.

7235. galeata Lcc.

7236. carinata Lece.

7237. xquicollis Esch.

\section{Cryptoglossa Sol.}

7238. verrucosa $L c c$.

7239. levis Lec.

\section{Centrioptera Mann.}

7240. spiculifera Lec.

7241. muricata Lec.

7242. asperata Horn.

7243. variolosa Horn.

7244. seriata $L c c$.

7245. infausta Lcc. 
Schizillus Horn.

7246. laticeps Horn.

\section{Microschatia Sol.}

7247. punctata Sol.

7248. inæqualis Lec.

7249. sulcipennis Lec.

7250. morata Horn.

\section{Ologlyptus Lac.}

7251. anastomosis Say.

\section{Astrotus Lec.}

7252. contortus Lec.

7253. regularis Horn.

\section{Asida Lat.}

7254. opaca Say.

7255. mancipata Horn.

7256. lirata Lec.

7257. polita Say.

7258. quadricollis Horn.

7259. sordida Lec.

7259a. costipennis Lec.

7259b. æger Lec.

$7259 c$. irregularis Lec.

7260. ægrota Lec.

7261. morbillosa Lec.

c 7262. actuosa Horn.

7263. semilavis Horn.

7264. carinata Lec.

7265. bifurca Lec.

7266. connivens Lec.

7267. confluens Lec.

7268. parallela Lec.

7269. 6-costata Lec.

7270. obsoleta Lec.

7271. muricatula Lec.

7272. hirsuta Lec.

7273. hispidula Lec.

7274. acerba Horn.

7275. luctata Horn.

7276. angulata Lec.

7277. Lecontei Horn.

7278. captiosa Horn.

7279. puncticollis Lec.

7280. consobrina Horn.
7281 convexa Lec.

7282 . obovata Lec.

7283. convexicollis Lec.

7284. marginata Lec.

7284a. rimata Lec.

7284b. subcylindrica Horn.

7285. Gabbii Horn.

7286. elata Lec.

7287. macra Horn.

\section{Branchus Lec.}

7288. fluridanus Lec.

\section{Coniontis Esch.}

7289. abdominalis Lec.

7290. robusta Horn.

elliptica Casey.

7291. opaca Horn.

7292. viatica Esch.

7293. puncticollis Lec.

7294. Eschscholtzii Mann.

7295. affinis Lec.

729ti. subpubescens Lec.

72!7. nemoralis Esch.

72!s. ovalis Esch.

7299. lata Lec.

73001. obesa Lec.

Colotaxis Horn.

7301. punctulata Horn.

7.:02. muricata Horn.

Cœlus Esch.

7303. ciliatus Esch.

7304. globosus Lec.

\section{Eusattus Lec.}

7305. robustus Lec.

7306. costatus Horn.

7307. reticulatus Say.

7308. erosus Horn.

7309. puberulus Lec.

7310. politus Horn.

7311. lævis Lec.

7312. dubius Lec.

7313. productus Lec.

7314. difficilis Lec.

7315. muricatus Lec. dilatatus Lec. 
Eleodes Eseh.

Discogenia Lec.

7316. obscura $S a y$.

7316a. dispersa Lec.

$7316 b$. suleipennis $\mathbf{Y a n n}$.

7317. aenta Say.

7318. suturalis Say.

$7318 a$. texana Lec.

7319. pedinoides Lec.

C 7320. tricostata Say.

7321. veterator Horn.

7322. carboriaria Say.

C 7323. obsoleta Say.

7324. quadricollis Esch.

c 7325. humeralis Lec.

7326. granulata Lec.

c 7327. extricata Say. arcuata Casey.

7328. militaris Horn.

C 7329. armata Lec.

73311. femorata Lec.

7331. Iongicollis Lec.

7332. gentilis Lec.

7333. ventricosa Lec.

7334. lucre Lec.

7335. gracilis Lec.

7336. innocens Lec.

7337. grandicollis Mann.

733\%. gigantea J Lann.

7339. nigrina Lec.

c 7340. hispilabris Say.

7341. sponsa Lec.

7342. caudifera $L c c$.

7343. acuticauda Lec.

73.4. dentipes Esch.

7345. granosa Lec.

7346. pilosa Horn.

7347. hirsuta Lec.

7348. scabripennis Lec.

C. 7349. clavicornis Esch.

7350. Lecontei Horn.

7351. consobrina Lec.

7352. tenebrosa Horn.

7353. planipennis Lec.

7354. parvicollis Esch.

7355. cordata Esch.

7356. pimelioides Mann.

c 7357. opaca Say.

7358. fusiformis $\mathrm{Lec}$.
7359. seriata Lec.

7360. subnitens Lec.

i361. striolata Lec.

7362. marginata Esch.

7363. scabricula Lec.

\section{Embaphion Say.}

C 7364. muricatum Say.

7365. contusum Lec.

73f6. elongatum Horn.

7367. planum Horn.

7368. depressum Lec.

\section{Trogloderus Lec.}

7369. costatus Lec.

Blaps Fab.

7370. mortisaga Linn.

\section{Eulabis Esch.}

7371. bicarinata Esch.

7372. grossa $L \varepsilon c$.

7373. rufipes Esch.

7374. pubescens Lec.

7375. obscura Lec.

\section{Cerenopus Lec.}

7376. concolor Lec.

7377. eribratus Lec.

7378. costulatus Horn.

\section{Argoporis Horn.}

7379. costipennis Lec. sulcipennis err. typ.

7350. bicolor Lec.

\section{Amphidora Esch.}

7381. littoralis Esch.

7382. nigropilosa Lec.

7383. tenebrosa Horn.

7384. caudata Horn.

\section{Cratidus Lec.}

7385. osculans Lec.

7386. rotundicollis Horn. 
Stenotrichus Lec.

7387. rufipes $L \epsilon c$.

Polypleurus Esch.

7388. geminatus Sol.

7389. perforatus Germ.

7390. nitidus $L c c$.

Nyctobates Guér.

$\forall$ 7391. penusylvanica $D e G$.

7391a. barbata Knoch.

7392. subnitens Horn.

Iphthimus Truq.

7393. opacus Lec.

7394. serratus Mann.

7394a. sublavis Bland.

7394b. Lewisii Horn.

7395. zopheroides Horn.

Cœlocnemis Mann.

7396. dilaticollis Mann.

7397. obesa Lec.

7398. magna Lec.

7399. punetata Lec.

Merinus Lee.

Ch 7400, lævis Oliv.

Upis Fab.

7401. ceramboides Linn.

Haplandrus Lec.

7402. femoratus $F a b$.

7403. ater Lec.

7404. concolor Lec.

\section{Centronopus Sol.}

7405. parallelus Lec.

\section{Cibdelis Mann.}

7406. Blaschkii Mann.

7407. Bachei Lec.

\section{Glyptotus Lec.}

7408. cribratus Lec.
Rhinandrus Lec.

7409. gracilis Lec.

7410. sublævis Horn.

Scotobates Horn.

$>$ 7411. culcaratus $F a b$.

7412. opacus Lec.

Xylopinus Lee.

c 7413. saperdioides Oliv.

7414. rufipes Say.

7415. renescens Lec.

Tenebrio Linn.

Tenebrionellus $\mathrm{Cr}$.

7416. obscurus Fab.

c 8 7417. molitor Linn.

7418. castaneus Knoch.

7419. tenebrioides Beauv.

-_ ? rufinasus Say.

Bius Muls.

7420. estriatus Lec.

Adelina Woll.

Sitophagus Muls.

7421. pallida Say.

7422. Lecontei Horn.

Alaephus Horn.

7523. pallidus Horn.

Eupsophus Horn.

7424. castaneus Horn.

Opatrinus Lat.

C 7425. notus Say.

C 7426. aciculatus Lec.

7427. Sayi Horn.

_- ? suturalis Say.

Mecysmus Horn.

7428. angustus Lec.

Blapstinus Lat.

7429. sordidus Lec. 
7430. latifrons Lec.

7431. sulcatus Lec.

7432. auripilis Horn.

c. 7433. dilatatus Lec.

7434. fortis Lec.

7435. opacus Lec.

7436. brevicollis Lec.

7437. Lecontei Muls.

C 7438. pratensis Lcc.

7439. vestitus Lec.

74t0. longulus Lec.

7441. discolor Horn.

A 7442. mestus Melsh.

7443. pulverulentus Mann. pimalis Casey.

7444. interruptus Say.

$>7445$. metallicus Fab.

7446. estriatus $L c c$.

Conibius Lec.

7447. seriatus Lec.

744S. parallelus Lec.

7449. elongatus Horn.

\section{Notibius Lec.}

7450. puberulus Lec.

7451. puncticollis Lec.

7452. gagates Horn.

7453. opacus Lec.

7454. granulatus Lec.

7455. suleatus Lec.

\section{Ulus Horn.}

745b. obliquus Lec.

7457. crassus Lec.

Cnemeplatia Costa.

7458. sericea Horn.

Alaudes Horn.

7459. singularis Horn.

\section{Ammodonus Muls.}

7460. fossor Lec.

Ephalus Lec.

7461. Iatimanus Lec.
Crypticus Lat.

Pedinus Lat.

7462. obsoletus Say.

Tribolium MacL.

74b3. ferrugineum $F a b$.

7464. madens Charp.

Diœdus Lee.

7465. punctatus Lec.

Phthora Muls.

7466. americana Horn.

Gnathocerus Thunb.

7467. cornutus Fab.

Echocerus Horn.

7468. maxillosus Fab.

Evoplus Lec.

7469. ferrugineus Lec.

Ulosonia Lap.

7470. marginata Lec.

Merotemnus Horn.

7471. elongatus Fiorn.

Mycotrogus Horn.

7472. piceus Horn.

7473. angustus Horn.

Aphanotus Lec.

7474. brevicornis Lec.

Alphitobius Steph.

7475. diaperinus Panz.

7476. piceus Oliv.

Cynæus Lec.

7477. angustus Lec.

7478. depressus Horn.

Metaclisa Duval.

7479. marginalis Horn. 
Tharsus Lec.

7480. seditiosus Lec.

\section{Uloma Lap.}

7481. impressa Melsh. 7482. imberbis Lec. 7483. mentalis Horn. 7484. longula Lec. 7485. punctulata Lec.

\section{Eutochia Lec.}

7486. picea Melsh.

7487. crenata Lec.

\section{Anædus Blanch.}

7488. brunneus Zieg7. 7489. rotundicollis $L c c$.

Paratenetus Spin.

7490, punctatus Sol.

7491. fuscus Lec.

_- cribratus Mots.

- gibbipennis Mots.

\section{Prateus Liec.}

7492. fusculus Lec.

\section{Phaleria Iat.}

7493. rotundata $L \in c$.

7494. limbata Horn.

7495. pilifera Lec.

7496. punctipes Lec.

7497. testacea Say.

7498. longula Lec.

7499. picipes Say.

7500. debilis Lec.

7501. globosa Lec.

7502. humeralis Horm. $\{a p$

7503. picta Mann.

\section{Anemia Lap.}

7504. californica Horn.

\section{Trachyscelis Lat.}

7505. flavipes Melsh.
Diaperis Geoff.

$\checkmark$ 7506. hydni Fab. 7507. rufipes Horn.

Hoplocephala Lap.

C. 7508. viridipenn is $\mathrm{Fab}$.

e. 7509 . bicornis Oliv.

Platydema Lap.

C 7510. excavatum Say. parvulum Casey.

7511. cyanescens Lap.

7512. erythrocerum Lap.

7513. ruficolle Lap.

Q 7514. ruficorne Sturm. opaculum Casey.

\& 7515. flavipes $F a b$.

7516. janus $F a b$.

7517. pernigrum Casey.

C. 751s. ellipticum Fab.

7519. oregonense Lcc.

7 7520. americanum Lap.

7521. lævipes Hald.

7522. crenatum Lec.

7523. micans Horn.

7524. picilabrum Melsh.

7525. subcostatum Lap.

__ cyaneum Lap.

-_ 4-maculatum Lap.

_- politum Lap.

Phylethus Meg.

7526. bifasciatus Say.

Liodema Horn.

7527. læve Hald.

Scaphidema Redt.

7328. æneolum $L c c$.

7529. pictum Horn.

\section{Hypophløus Fab.}

75̄30. substriatus Lec.

7531. cavus Lec.

7532. parallelus Melsh.

7533. glaber Lec.

7534. tenuis Lec.

7535. thoracicus Melsh. 
7536. piliger Lec.

7537. opaculus Lec.

Pentaphyllus Meg.

7538. pallidus Lec.

7539. californicus Horn.

Boletotherus Cand.

Q 7540. bifurcus Fab.

\section{Boletophagus Ill.}

7541. corticola Say.

7542. depressus Rand.

\section{Apocrypha Esch.}

7543. anthicoides Esch.

7544. dyschirioides Lec.

7545. clivinoides Horn.

\section{Helops Fab.}

7546. micans Fab.

7547. viridimicans Horn.

7548. americanus Beauv. undulatus Lec.

7549. venustus Say.

7550. impolitus Lcc.

7551. opacus Lec.

7552. punetatus Gemm.

7553. rugulosus $L e c$.

7554. angustus Lce.

7555. gracilis Bland.

7556. californicus Mann.

7557. Edwardsii Horn.

7558. pernitens Lec.
7559. letus $L e c$.

7560. rugieollis Lec.

7561. strigicollis Horn.

7562. attenuatus Lec.

7563. Bachei Lec.

7564. convexulus Lcc.

7565. æerens Germ.

7566. suleipennis Lec.

7567. perforatus Horn.

7568. arizonensis Horn.

7569. difficilis Horn.

7570. discretus Lec.

7571. eisteloides Germ.

7572. spretus Horn.

7573. faretus $L \in C$.

7574. tumescens Lec.

__ politus Say.

- tristis Beauv.

Dignamptus Lec.

7575. langurinus Lec.

7576. stenochinus Lec.

\section{Pyanisia Lap.}

7577. opaea Sol.

\section{Meracantha Kirby.}

Ch 7578. contracta Beauv.

\section{Strongylium Kirby.}

7579. tenuicolle Say.

7580. terminatum Siry.

7581. anthrax $S z$.

7582. simplicicolle Lec.

7583. crenatum Makkl.

\section{EGIALITID E.}

Amgialites Mann.

7584. debilis Mann.

\section{CISTELIIDE.}

Prostenus Lat.

7585. ealifornicus Horn.

\section{Stenochidus Lec.}

7isfi. gracilis Lec.

7.57. cyanescens Lec.
Allecula Fab.

7588. erythrocnemis Germ.

7589. punctulata Melsh.

7590. nigrans Melsh. 
Hymenorus Muls.

7591. pilosus Melsh.

7592. obscurus Say.

759\%. punctulatus Lec.

7594. niger Melsh.

7595. communis Lec.

7596. rufipes $L e c$.

7597. confertus Lec.

7598. densus Lec.

75!9. punctatissimus Lec.

7600. humeralis Lec.

ib01. dorsalis $S z$.

\section{Cistela Fab.}

7602. brevis Say.

7603. pinguis Lec.

7604. opaca Lec.

7605 . Thevenetii Horn.

7606. marginata Ziegl.

$\$ 7607$. sericea Say.

7608. variabilis Horn.

_- pulla Melsh.

Isomira Muls.

7609. quadristriata Coup.

7610. valida $S z$.

7611. velutina Lec.

Mycetochares Lat.

7612. rufipes Lee.

\section{Othnnius Lec.}

7633. umbrosus Lec.

7634. lugubris Horn.
7613. pubipennis Lec.

7614. laticollis Lec.

7615. Haldemani Lec.

7616. fraterna Say.

7617. foveata Lec.

7618. tenuis Lec.

7619. bicolor Coup.

7620 . gracilis Lec.

7621 . analis Lec.

7 7i22. lugubris Lec.

7623. marginata Lec.

7624. binotata Say.

762.;. longula Lec.

7626 . basillaris Say.

-_ rufieornis Melsh.

Chromatia Lec.

7627. amœna Say.

Capnochroa Lec.

$>$ 7628. fuliginosa Melsh.

Androchirus Lec.

$>$ 7629. fuscipes Melsh.

7630. femoralis Oliv.

7631. erythropus Kirby. luteipes Lec.

\section{Cteniopus Sol.}

7632. Murrayi Lec.

\section{O'W I I I I I AE.}

7635. longicornis Horn.

7636. fasciatus Bland.

\section{CA GIR I I) $A$.}

Arthromacra Kirby.

c 7637. ænea Say.

Statira Lat.

7639. resplendens Melsh.

7640. gagatina Melsh.

7641. subnitida Lec.

7638. croceicollis Mäkl.

\section{MONOMMIDE.}

Hyporhagus Thom.

7642. punctulatus Thom.

7643. opaculus Lec.
7644. opuntix Horn.

7645. gilensis Horn. 


\section{MELANDRYIID.}

Tetratoma Fab.

7646. truncorum Lec.

7647. tessellata Melsh.

7648. concolor Lec.

Penthe Newm.

7 7049. obliquata $\mathrm{Fab}$.

$>7650$. pimelia $F a b$.

\section{Synchroa Newm.}

7651. punctata Newm.

Prothalpia Lec.

7652. undata Lee.

Melandrya Fab.

Y. 7653. striata Say.

Emmesa Newm.

7654. connectens Newm.

7655. labiata Say.

\section{Phryganophilus Sahlb.}

7656. collaris Lec.

? depressus $F a b$.

Amblyctis Lee.

7657. præses Lec.

Xylita Payk.

7658. lævigata Hellw. decolorata Rand.

Zilora Muls.

7659. hispida Lec.

7660. nuda Prov.

Carebara Lec.

7661. longula Lec.

Spilotus Lec.

7662. quadripustulosus Melsh.

Scotochroa Lec.

7663. atra Lec.
7664. basalis Lec.

Enchodes Lec.

7665. sericea Hald.

Serropalpus Hellw.

7666. barbatus Schall. striatus Hellw.

Hypulus Payk. Mystaxis Kug.

7667. simulator Newm.

Marolia Muls.

7668. fulminans $L e c$.

Dircæa Fab.

7669. liturata Lec.

7670. prona Lec.

7671. fusca Lec.

7672. coneolor Lec.

7673. Riversi Lec.

Symphora Lec.

7674. flavicollis Hald.

7675. rugosa Hald.

Anisoxya Muls.

7676. glaucula Lec.

Eustrophus III.

7677. indistinetus Lec.

7678. confinis Lec.

7679. bicolor Say.

7680. bifasciatus Say.

7681. tomentosus Say.

7682. impressicollis Lec.

Hallomenus Panz.

7683. scapularis Melsh.

7684. punctulatus Lec.

? basalis Mann.

7685. serricornis Lec.

7686. debilis Lec. 
Orchesia Lat.

7687. castanea Melsh.

7688. gracilis Melsh.

Microscapha Lee.

7689. clavicornis Lec.

Scraptia Lat.

7690. sericea Melsh.

Allopoda Lec.

7691. Iutea Hald.

Canifa Lec.

7692. plagiata Melsh.

7693. pusilla Hald.

7694. pallipennis Lec.

7695. pallipes Melsh.

Stenotrachelus Lat.

7696. arctatus Say.

Sphalma Horn.

7706. quadricollis Horn.

\section{Crymodes Lec.}

7707. discicollis Lec.

\section{Boros Hbst.}

7708. unicolor Say.

Pytho Lat.

7709. niger Kirby.

87710 . americanus Kirby.

7711. deplanatus Mann.

7712. strictus Lec.

Priognathus Lec.

7713. monilicornis Rand.
Scotodes Esch.

7697. americanus Horn.

Nothus Oliv.

7698. varians Lec.

7699. luteus Horn.

Lacconotus Lec.

7700. punctatus Lec.

7701. pinicolus Horn.

Mycterus. Clairv.

7702. canescens Horn.

7703. scaber Hald.

7704. concolor Lec.

$7704 a$. Alavipennis Horn.

7705. quadricollis Horn.

\section{PYTHIDA.}

Cononotus Lec.

7714. macer Horn.

7715. punctatus Lcc.

7716. sericans Lec.

Salpingus Gyll.

7717. virescens Lec.

7718. alternatus Lec.

7719. tibialis Lec.

7720. elongatus Mann.

Rhinosimus Lat.

7721. æneirostris Mann.

7722. pallipes Boh.

7723. viridiæneus $R$ and.

\section{GDEYERID E.}

\section{Calopus Fab.}

7724. angustus Lec.

7725. aspersus Lec.

\section{Microtonus Lec.}

7726. sericans Lec.
Ditylus Fisch.

7727. cæruleus Rand. 7728. quadricollis Lec. 7729. gracilis Lec. 7730. obscurus Lec. 7731. bicolor Horn. 7732. cyanipennis Horn. 
Nacerdes Schm.

$Q$ 7733. melanura Linn.

Xanthochroa Schm.

7734. lateralis Melsh.

7735. trinotata Lec.

7736. californica Horn.

\section{Copidita Lec.}

7737. quadrimaculata Mots.

\section{Rhinoplatia Horn.}

7738. ruficollis Horn.

Oxacis Lec.

7739. cana Iec.

7740. pallida Lec.

7741. treniata Lec.
7742. thoracica $F a b$.

7743. sericea Horn.

7744. notoxoides $F a b$.

7745. granulata $L e c$.

7746. bieolor Lec.

7747. fuliginosa Lec.

7748. dorsalis Melsh.

\section{Probosca Schm.}

7749. Iucana $L e c$.

7750. pleuralis Lec.

Așclera Schm.

7751. excavata Lec.

7 7752. ruficollis Say.

7753. puncticollis Say.

7754. erythrocephala Germ.

7755. nigra Lec.

7756. discolor $L c c$.

- ? rufifrons $F u b$. .

\section{CHPIIAOIDA.}

Cephaloon Newm.

7757. lepturides Newm.
7758. ungulare Lec.

7759. tenuicorne Lec.

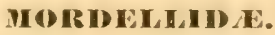

Diclidia Lec.

7760. Lretula Lec.

Pentaria Muls.

77ili. trifasciata Melsh.

Fride fuscula Lec.

776i3. nubila Lec.

7764. hirsuta Smith.

Anaspis Geoti".

$\begin{array}{ll}7765 . & \text { nigra Hald. } \\ 7766 . & \text { atra Lec. } \\ 7767 . & \text { militaris Smith. } \\ 7768 . & \text { sericea Mann. } \\ & \text { luteipennis Lec. } \\ 7769 . & \text { flavipennis Hald. } \\ 7770 . & \text { rufa Say. } \\ & \text { nigriceps Lec. } \\ 771 . & \text { pusio Lec. } \\ 772 . & \text { collaris Lec. }\end{array}$

Tomoxia Costa. Glipa Lec.

7773. bidentata Say.

7774.' lineella Lec.

7775. inclusa Lec.

7776. hilaris Say.

\section{Mordella Linn.}

7777. quadripunetata Say.

7778. borealis $L \varepsilon c$.

7779. melæna Germ.

4780 . scutellaris $F a b$.

7781. inflammata Lec.

7782. octopunetata Fab.

77s3. marginata Melsh. lineata Melsh.

7784. lunulata Helm. obliqua Lec.

7784a. deserta Casey.

7785. serval Say.

7786. angulata Lec. 
7787. oculata Say. jovialis Lec.

7788. insulata Lec.

7789. triloba Say.

7790. undulata Melsh.

7791. fascifera Lec.

7792. discoidea Melsh.

- atrata Melsh.

dimidiata Melsh.

modesta Melsh.

pubescens $F a b$.

\section{Glipodes Lec.}

7793. sericans Melsh.

7794. helva Lec.

\section{Mordellistena Costa.}
7795. bicinctella Lec.
7796. arida Lec.
7797. lutea Melsh.
7798. trifasciata $S a y$. nigricollis Helm.
7799. lepidula Lec.
7800. limbalis Melsh.
7801. elegantulus Smith.
7802. dimidiata Helm.
7803. biplagiata Helm.
7804. intermixta Helm.
7805. vilis Lec.
7806. vapida $L e c$.
7807. decorella Lec.
7808. bipustulata Helm.
7809. atriceps Smith.
7810. picipennis Smith.
7811. pallipes Snith.
7812. fulvicollis Melsh.
781n. ornata Melsh.
7814. militaris Lec.
7815. seapularis Say.
7816. comata Lec.
cervicalis Lec.
picicornis Lec.
7817. aspersa Melsh.
7818. indistincta Smith
7819. tosta Lec.
7820. inornata Smith.
7821. amica Lec.
7822. minuta Smith.

7823. picilabris Helm.

7824. infima $L e c$.

7825. andreæ Tec.

7826. grammica Lec.

7827. ancilla Lec.

7828. varians Lec.

7829. ustulata Lec.

7830. seminsta Lec.

7831. impatiens Lec.

7832. ferruginoides Smith.

7833. nigricans Melsh.

7834. guttulata Helm.

78.35. ruficeps Lec.

7836. nubila Lec.

7837. splendens Smith.

7838. floridensis Smith.

7839. pustulata Melsh.

7840. convicta Lec.

77841. fuscipennis Melsh.

7842. pectoralis Lec.

7843. morula Lec.

7844. ambusta tec.

7845. singularis Smith.

7846. unicolor Lec.

7847. marginalis Say. divisa Lec.

7848. Schauppii Smith.

7849. æqualis Smith.

7850. scalaris Helm.

7851. pubescens Fab. hebraica Lec. leporina $L e c$.

7\$52. bihamata Melsh.

7853. liturata Melsh.

7851. fuscata Melsh.

785.). pityptera Lec.

7isti. rufescens Smith.

7557. cinereo-fasciata Smith.

is.s. æ. æhiops Smith.

7859. texana Smith.

7860. fusco-atra Helm.

7861. suturella Helm.

7862. rufiventris Helm.

786.) angusta Lec.

7864. attenuata Say. vittigera Lec.

7865. discolor Melsh.

78666. æmula Lec.

7\&67. nigripennis Fab.

__ ? rufula Helm. 


\section{NTHICIDA.}

\section{Nematoplus Lec.}

7868. collaris Lec.

\section{Eurygenius Laf.}

7869. Wildii Lec.

7870. murinus Hald.

7871. constrictus Lec.

7872. campanulatus Lec.

\section{Stereopalpus Laf.}

7873. Mellyi Laf.

7874. badiipennis Lec. .

7875. guttatus Lec.

7876. pruinosus Lec.

__ vestitus Say.

\section{Bactrocerus Lec.}

7877. eoneolor Lec.

\section{Corphyra Say.}

7878. flabellata Horn.

7879. Crotehii Horn.

7880. abnormis Horn.

7881 . distinguenda Horn.

7882. Bardii Horn.

7883. fiunebris Horn.

7884. punctulata Lec.

7885. canalieulata Lee.

7856. elegans Hentz.

7887. terminalis Say.

7888. Lewisii Horn.

7885a. monticola Horn.

7889 . fulvipes Newm.

7890. Newmani Lec.

7891. eyanipennis Bland.

7892. pulchra Lec.

7893. labiata Say.

7894. lugubris Say.

7895. enllaris Say. -

7896. inconspicua Horn.

7897. vittata Horn.

\section{Xylophilus Lat.}

7898. Melsheimeri Lec.

7899. basalis Lec.

7900. nebulosus Lec.
7901. ater Lec.

7902. fasciatus Melsh.

7903. nubifer Lec.

7904. subfasciatus Lec.

7905. signatus Hald.

7906. notatus $L e c$.

7907. ventricosus $L e c$.

7908. ptinoides $S z$.

7909. quercicola $\mathbf{S z}$.

7910. brunnipennis Lec.

7911. piceus Lec.

7912. densus Casey.

7913. impressus Lec.

Macratria Newm.

7914. confusa Lec.

7915. murina Fab.

\section{Notoxus Geoff.}

7916. bicolor Say.

7917. nuperus Horn.

7918. talpa Laf. elegantulus Lec. subtilis $L c c$.

7919. bifasciatus Lec.

7920. planicornis Laf.

7921. apicalis $L e c$. marginatus Lec.

7922. calcaratus Horn.

792:?. monodon Fab.

Pilatei Laf. delicatus Casey.

7924. serratus $L e c$. digitatus Lec.

7!25. anchora Hentz.

7926. denudatus Horn.

7927. conformis Lec. sparsus Lec.

792s. cavicornis Lec.

Mecynotarsus Laf.

7929. delicatulus Horn.

7930. candidus Lec.

7931. elegans $L e c$.

\section{Tomoderus Laf.}

7932. interruptus Laf. 7933. constrictus Say. 


\section{Formicomus Laf.}

7934. seitulus Lec.

7935. mundus Lee.

\section{Anthicus Payk.}

7936. obscurus Laf.

7937. nitidulus Lec.

7938. Sturmii Laf. elegans Lif.

7939. tenuis Lec.

7940. formicarius Laf.

7941. cinctus Say.

7942. annectens Lec.

7943. californicus Laf.

7944. rejectus $L e c$.

7945. Horalis Linn.

7946. vicinis Laf.

7947. thoracicus Laf.

7948. confinis $L e c$.

7949. horridus Lec.

7950. cribratus Lec.

7451. difficilis Lec.

7952. confusus Lec.

7953. luteolus Lec.

7954. nigrita Mann.

7955. scabriceps Lec.

7956. ephippium Laf.

7957. flavicans Lec.

7958. rufulus Lec.

7959. cervinus Laf.

7960. punctulatus Lec.

7961. Haldemani Lec.

7962. quadrilunatus Laf.

7963. biguttulus Lec.

7964. nigritulus Lec.
7965. obscurellus Lcc.

7966. latebrans Lec.

7967. spretus $L e c$. facilis Casey.

7968. nanus Lec.

7969. bellulus Lec.

7970. pubescens Lec.

7971. fulvipes Laf.

7972. corticalis Lec.

7973. maritimus Lec.

7974. ictericus Laf.

7975. coracinus Lec.

7976. pallens Lec.

7977. granularis Lec.

7978. exilis Laf.

7979. lætus Laf.

7980. melancholicus Laf.

7981. pusillus Laf.

7982. squamosus $L a f$.

7983. lugubris Laf.

7984. impressipennis Laf.

7985. texanus Laf.

7986. pallidus Say.

_- ? politus Say.

___ ? amplicollis Boh.

- ? atomarius Boh.

- ? eæsiosignatus Boh.

__ ? nitidus Boh.

_- ? troglodytes Boh.

Tanarthrus Lec.

7987. salinus Lec.

7988. salicola Lec.

7989. alutacens Lec.

\section{PY YOCHIROIDE.}

\section{Ischalia Pasc.}

7990. costata Lec.

\section{Pyrochroa Geoff.}

7991. flabellata $F u b$.

7992. femoralis Lec.

\section{Schizotus Newm.}

7993. cervicalis Newm.

\section{Dendroides Lat.}

7994. canadensis Lat.

7995. concolor Newm.

7996. picipes Horn.

7997. ephemeroides Mann. testacea Lec. 


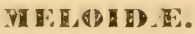

Cysteodemus Lec.

7998. armatus Lic.

$>$ 7999. Wislizeni Lec.

Megetra Lec.

$\$ 000$. vittata $L c c$. cancellata $\ddagger$ Lec.

8001. opaca Horn.

Meloe Linn.

S002. montanus Ler.

s003. tinetus Lec.

S004. carbonaceus Lec.

S005. afer Bland.

S006. impressus Kirby.

S007. niger Kirby.

soos. npacus Lcc.

8009. barbarus Lec.

S010. perplexus tee.

S011. strigulosus Mann.

S012. angusticollis Say.

8013. americanus Leach.

s014. morens Lcc.

S015. sublrevis Lcc.

Nomaspis Lee.

So10. parvula Hald.

8017 . sublrevis Horn.

Henous Hald.

c S018. confertus Say.

Poreospasta Horn.

S019. polita Horn.

\section{Horia Fab.}

S020. maculata Swed.

Tricrania Lee.

8021. Sanguinipennis $S a y$.

S022. Stansburii Huld.

s023. Murrayi Lec.

Nemognatha IIl.

8024. Iurida Lec. decipiens Lec.
8025. apicalis $L c c$.

8026. lutea Lec. pallens $L \varepsilon c$.

8027. bicolor Lec.

8028. dichroa Iec.

8029. dubia Lec.

8030. palliata Lec.

广 8031. piezata Fab. H texana Lee.

8032. punctulata Lec.

- flavipennis Uhler.

8033. nigripennis Lec.

s034. nemorensis Hentz.

8035. cribraria Lec.

S0.36. sentellaris Lec.

s037. immaculata Say.

8038. punctipenn is Lec.

8039. sparsa Lee.

$7 \mathrm{~s} 040$. vittigera Lec.

8041. eribrieollis Lec. porosa Lec. ? calceola Gućr.

\section{Gnathium Kirby.}

8012. Francilloni firby.

s043. minimum Say.

8044. lougicolle Lec

8045. texanum Horn.

s046. nitidum Horn.

Zonitis Fab.

80.4. atripennis Say.

S048. Havida Lec.

8049. bilineata Say.

S050. longicornis Horn.

8051. rufil Lec.

8052. vittipennis Horn.

Hornia IRiley.

8053. minutipennis Ruley.

Cordylospasta Horn.

8054. Fulleri Horn.

\section{Macrobasis Lee.}

8055. albida Say.

S056. longicollis Lec. sublineata Lec. 
S057. atrivittata Lec.

5058. purpurea Horn.

8059. virgulata Lec.

c

8060. tort́a Lec.

8061. unicolor Kirby.

8062. tenuis Lec.

8063. linearis Lec.

5064. ochrea Lec.

8065. Gissleri Horn.

8066. lauta Horn.

8067. tenella Lec.

8068. immaculata Say.

c 8069. segmentata Say.

\section{Tetraonyx Lat.}

8070. frontalis Chev.

8071. femoralis Dugés.

S072. fulva Lec.

8073. quadrimaculata Fab.

\section{Pleuropompha Lec.}

8074. costata Lec.

\section{Gnathospasta Horn.}

8075. minetica Horn.

\section{Epicauta Redt.}

8076. Alphonsii Horn.

8077. puncticollis Mann.

8078. oblita Lec.

8079. trichrus Pall.

s080. strigosa Gyll.

8081. Batesii Horn.

y 8082. ferruginea Say.

8083. Eericans Lec.

8084. pruinosa Lec.

8085. callosa Lec.

8086. Rileyi Horn.

8087. caviceps Horn.

8088. pedalis Lec.

8089. insignis Horn.

S090. nigritarsis Lec.

8091. pardalis Lec.

C 8092. maculata Say.

C S093. vittata Fab.

8094. lemniscata $F a b$.

8095. Stuarti Lec.

8096. Wheeleri Ulke.

8097. cinerea Forst.
8098. cupreola Dugés

8099. oregona Horn.

8100. funebris Horn.

8101. fissilabris Lec.

8102. fallax Horn.

8103. corvina Lec.

C 8104. pennsylvanica $D e G$.

8105. maura Lec.

8106. sanguinıcollis Lec.

\section{Pyrota Lec.}

C 8107. mylabrina Chev.

8108. terminata Lec.

8109. Engelmanni Lec.

8110. postica Lec.

8111. dubitabilis Horn. rufipennis $\ddagger$ Lec. vittigera $\|$ Lec.

8112. insulata Lec.

8113. Germari Hald.

8114. discoidea Lec.

8115. invita Horn.

8116. limbalis Lec.

8117. lineata Oliv.

8118. bilineata Horn.

8119. sinuata Oliv. Afzeliana $F a b$.

\section{Pomphopœa Lec.}

8120. polita Say.

8121. Sayi Lec.

8122. unguicularis Lec.

8123. texana Lec.

8124. ænea Say.

8124a. tarsalis Bland.

\section{Cantharis Linn.}

8125. eucera Chev.

8126. mutilata Horn.

8127. 4-maculata Chev.

8128. melæna Lcc.

8129. magister Horn.

8130. deserticola Horn.

8131. vulnerata Lec.

8131 $\%$. Cooperi Lec.

C 8132. Nuttalli Say.

8133. cyanipennis Say.

8134. viridana Lec.

8135. lugubris Ulke. 
8136. Childii Lec.

8137. tenebrosa Lec.

8138. puberula Lec.

8139. biguttata Lec.

8140. fulvipennis Lec. cardinalis $\ddagger L e c$.

8141. gentilis Horn.

8142. mœrens Lec.

8143. insperata Horn.

8144. molesta Horn.

8145. reticulata Say.

8146. cribrata Lec.

8147. dichroa Lec.

8148. occipitalis Horn.

8149. incommoda Horn.

8150. stygica Lec.

8151. auriculata Horn.

8152. refulgens Horn.

8153. Crotehii Horn.

8154. seneipennis Lec.

8155. nitidieollis Lec.

8156. lugens Lec.

8157. Rathvoni Ler.
8158. sphrericollis Say.

8159. compressicornis Horn.

8160. convexa Lcc.

Calospasta Lec.

8161. elegans Lec.

8161n. humeralis Horn.

8162. perpulchra Horn.

8163. Fulleri Horn.

8164. mirabilis Horn.

8165. mesta Horn.

8166. nemognathoides Horn.

8167. viridis Horn.

Tegrodera Lec.

81fi8. erosa $L c c$.

Eupompha Lec.

8169. fissiceps Lec.

Phodaga Lee.

\$170. alticeps Lec.

\section{RHI I PI PII O IR I I N.}

Pelecotoma Fisch.

8171. flavipes Melsh.

\section{Toposcopus Lec.}

8172. Wrightii Lec,

\section{Rhipiphorus Fab.}

8173. flavipennis Lec.

8174. dimidiatus $F u b$.

8175. bifoveatus Horn.

817ti. octomaculatus Gerst.

8176a. puncticeps Lec.

S176ib. Sayi Lec.

8177. pectinatus $\mathrm{Fal}$. militaris Mels. niger Mels.

ventralis $F a b$.
8178. cruentus Germ.

8178 a rufus Lec.

8179. limbatus $F u b$.

8180. linearis Lec.

\section{Myodites Lat.}

8181. Popenoi Lec.

8182, semiflavus Lec.

8183. scaber Lec.

8184. luteipennis $L c c$.

8185. nevadicus Lec.

8186. californicus $L e c$.

8178. fasciatus Say.

8188. Schwarzi Lec.

8189. Zeschii Lec.

8190. Walshii Lec.

8191. stylopides Newm.

8192. Hlavicornis Say.

8193. americanus Guér.

S'TY YOPU D.:

Stylops Kirby.

81!4. Childreni Gray.
Xenos Rossi.

8195. Peckii Kirby. 


\section{RHI NOM A CERID.}

Rhinomacer Fab.

8196. pilosus Lec.

Diodyrhynchus Sch.

8197. elongatus Lec.

8200. byturnides $L \ell c$.

8198. comptus Lec.

8199. bombifrons Lec.

\section{RHY NCHITI D.}

Auletes Sch.

8201. ater Lec.

8202. nasalis Lec.

82n3. subcreuleus Lec.

8204. cassandræ Lec.

\section{Eugnamptus Sch.}

8205. striatus Lec.

8206. angustatus $\mathrm{Hbst}$.

8207. collaris Fab.

8208. puncticeps Lec.

8209. sulcifrons Gyll.

Rhynchites Hbst.

8. 8210. bicolor Fab.

C. 8211. æneus Boh.

8212. mexicanus Gyll.

8213. eximius Lec.

8214. velatus Lec.

C 8215. hirtus Fab.

8216. glastinus Lec.

8217. planifrons Lec.

8218. aureus $L e c$.

8219. fossifrons $L c c$.

8220. naso Casey.

8221. cyanellus Lec.

8222. æratus Say.

-C ? humeralis Boh.

_- ? congruus Walk.

Pterocolus Sch.

yे 8223 . ovatus Fab.

\section{ATTELA BIDE.}

Attelabus Linn.

8224. analis Ill.

8225. nigripes Lec.

C 8226. bipustulatus Fab. 8227. genalis Lec.

y 8228 . rhois Boh.

IS Y RSOPID A .

Thecesternus Say.

C 8229. humeralis Say.

\section{O'TIORH Y NCHIDA.}

Minyomerus Horn.

8230. innocuus Horn.

8231. languidus Horn.

Graphorhinus Sch.

8232. vadosus $S a y$.

Epicærus Sch.

C 8233. imbricatus Say. 8234. formidolosus Boh.

Anomadus Horn.

8235. obliquus Horn.
Barynotus Germ.

8236. Schœnherri Zett.

__ ? granulatus Say.

Hormorus Horn.

8237. undulatus Uhler.

Agasphærops Horn.

8238. nigra Horn.

Brachyderus Sch.

8239. incanus Linn. 
Trigonoscuta Mots.

S240. pilosa Mots.

Calyptillus Horn.

8241. cryptops Horn.

Ophryastes Sch.

c 8242. vittatus Say. 8243. tuberosus Lec.

8244. latirostris $L e c$.

8245. sulcirostris Say.

8246. porosus Lec.

\section{Eupagoderes Horn.}

8247. speciosus Lec.

S248. decipiens $L \varepsilon c$.

8249. sordidus Lec.

8250. argentatus Lec.

8251. lucanus Horn.

8252. desertus Horn.

8253. varius Lec.

S254. geminatus Horn.

8255. plumbeus Horn.

Rhigopsis Lec.

8256. efiracta Lec.

Dichoxenus Horn.

8257. setiger Horn.

Anametis Horn.

8258. grisea Horn.

Melamomphus Horn.

8259. niger Horn.

Dyslobus Lec.

S260. segnis Lec.

Panscopus Sch.

8261. erinaceus Say.

Orimodema Horn.

8262. protracta Horn.

Mimetes Sch.

S263. setulosus $\mathcal{L}$ C s2fit. seniculus Horn.
Diamimus Horn.

8265. subsericeus Horn.

Peritaxia Horn.

Y) 8266. rugicollis Horn.

C 8267. hispida Horn.

Thricomigus Horn.

8268. luteus Horn.

Amnesia Horn.

S269. granicollis Lcc.

8270. decorata $L \varepsilon c$.

8271. ursina Horn.

8272. rauca Horn.

8273. alternata Horn.

8274. sordida Horn.

8275. decidua Horn.

S276. elongata Horn.

Phymatinus Lec.

8277. gemmatus Lec.

Nocheles Horn.

4278. torpidus Lec.

8279. xqualis Horn.

Cimbocera Horn.

8280. pauper Horn.

Phyxelis Sch.

ch 8281. rigidus Say.

Agraphus Sch.

8282. bellicus Say.

Otiorhynchus Germ.

8283. sulcatus Fab.

C 8284. ovatus Linn. ligneus $\ddagger L c c$.

8285. rugifrons Gyll.

S28fi. maurus Gyll.

8287. monticola Germ.

Sciopithes Horn.

8288. obscurtis Horn. 
Agronus Horn.

8289. deciduus Horn.

8290. cinerarius Horn.

\section{Neoptochus Horn.}

8291. adspersus Boh.

Paraptochus Seidl.

8292. sellatus Boh.

Mylacus Sch.

8293. saceatus Lec.

Thricolepis Horn.

8294. inornata Horn.

8295. simulator Horn.

Peritelopsis Horn.

8296. globiventris $L e c$.

Geoderces Horn.

8297. melanothrix Kirby. 8298. incomptus Horn.

Aragnomus Horn. 8299. griseus Horn.

\section{Dysticheus Horn.}

8300. insignis Horn.

\section{Eucyllus Horn.}

8301. vagans Horn.

\section{Thinoxenus Horn.}

8302. squalens Horn.

\section{Rhypodes Horn.}

8303. dilatatus Horn.

8304. brevicollis Horn.

\section{Cercopeus Sch.}

8305. chrysorhous Say.

\section{Chætechus Horn.}

8306. setiger Horn.

Trachyphlœus Germ.

8307. asperatus Boh.
Dirotognathus Horn.

8308. sordidus Horn.

Pachnæus Sch.

8309. opalus Oliv.

8310. distans Horn.

Tanymecus Sch.

8311. lacæna Hbst.

C8312. confertus Gyll.

Hadromerus Sch.

8313. opalinus Horn.

Pandeletejus Sch.

> 8314. hilaris Hbst.

8315. cinereus Horn.

Compsus Sch.

$>$ 8316. auricephalus Say.

Cyphus Germ.

7 831\%. lautus Lec.

8318. placidus Horn.

Brachystylus Sch.

8319. acutus Say.

Artipus Sch.

8320. floridanus Horn.

Aramigus Horn.

c 8321. tesselatus Say. 8322. Fulleri Horn.

Phacepholis Horn.

8323. elegans Horn.

8324. obseura Horn.

8325. candida Horn.

Achrastenus Horn.

8326. griseus Horn.

Aphrastus Sch.

C327. tæniatus Gyll. 8328. unicolor Horn. 
Lachnopus Sch.

8329. Horidanus Horn.

Omileus Horn.

8330. epicæroides Horn.

Evotus Lec.

8331. naso Lec.

\section{Phyllobius Germ.}

8332. calcaratus Fab.

Cyphomimus Horn.

8333. dorsalis Horn. ? americanus Gyll.

Scythropus Sch.

8334. elegans Coup. metallicus Desb.
S335. californicus Horn.

Mitostylus Horn.

8336. tenuis Horn. .

Coleocerus Sch.

8337. dispar Lec.

8338. marmoratus Horn.

Aracanthus Sch.

8339. pallidus Say.

Eudiagogus Sch.

$>$ 8340. pulcher Fuh.

8. 41. Rosenschœldi Fuh.

Promecops Sch.

- arcuata $F a b$. nubifera Gyll.

\section{Y CUIRCULIONIDA.}

Sitones Sch.

c 8342. lineellus Gyll. 8343. ealifornicus $F u h$.

8344. sordidus Lec.

8345. vittatus Lec.

8346. hispidulus Germ.

17 8347. flavescens Marsh.

8348. tibialis Hbst.

8349. crinitus Oliv.

Triglyphus Lec.

8350, ater Lec.

\section{Plinthodes Lec.}

8351. taniatus Lec.

Acmæegenius Lec.

8352. hylobinus Lec.

Trichalophus Lec.

8353. didymus Lec.

Sis. 4 . constrictus Lec.

$\therefore: 55$. alternatus Say.

\$356. seriatus Mann.

8357. simplex Lec.

8358. planirostris Lec.
Lophalophus Lec.

8359. inquinatus Mann.

Lepidophorus Kirby.

8360. lineaticollis Kirby.

Ithycerus Sch.

y S361. noveboracensis Forst.

Apion Hbst.

S362. erraticum Smith.

8363. obsoletum Smith.

8364. erythrocerum Smith.

8365. robustum Smith.

8366. protensum Lec.

8367. punetinasum Smith.

836s. parallelum Smith.

8369. estriatum Smith.

8370. ()vile Smith.

8:31. impunctistriatum Smith.

S372. desolatum Smith.

8373. sordidum Smith.

8374. floridanum Smith.

8375. obesum Smilh.

8376. parvulum Smith.

8377. atripes Smith. 
8378. eribricolle Lec.

C 8379. melanarium Gerst.

8380. minutum Smith.

8381. pennsylvanicum Boh.

8382. opacicolle Smith.

8383. adorhynchum Lee.

8384. texauum Simith.

8385. elliptieum Smith.

8386. californicum Smith.

8387. vespertinum Casey.

8388. decolnratum Smith.

8389. concoloratum S'mith.

8390. carinatum Smith.

8391. brevicolle Smith.

8392. typicum Smith.

8393. abdominale Smith.

8394. antennatum Smith.

8395. cordatum Smith.

8396. capitatum Smith.

8397. oblitum Smith.

8398. ventricosum Lec.

8399. subglobosum Gerst.

8400. turbulentum Smith.

8401. minor Smith.

8402. dilatatum Smith.

C 8403. herculanum Smith.

8404. pyriforme Smith.

8105. Walshii Smith.

lanuginosum Walsh.

8406. vicinum Smith.

8407. crassinasum Lec.

8408. proclive Lec.

8409. modestum Smith.

8410. perminutum Smith.

8411. segnipes Say. cipereum Gerst.

8412. griseum Smith.

8413. fraternum Smith.

8414. varicorne Smith.

8115. troglodytes Mann. metallicum Gerst.

8416. extensum Smith.

8417. æneipenne Smith.

8118. contusum Smith.

8419. tenuirostrum Smith.

8420. attenuatum Srnith.

8421. cavifrons Lec.

8422. confertum Smith.

8123. rostrum Say.

8424. nigrum Hbst.
-8125. patrilele Smith.

- porcatum Boh.

- cuprescens Mann.

- nodirostre Gerst.

-..- reconditum Gyll.

- vile Gerst.

\section{Podapion Riley.}

8426. gallicola Riley.

\section{Phytonomus Sch.}

8127. punctatus $F a b$. opimus Lec.

8128. elongatus Payk.

8429. setigerus Lec.

\$430. comptus Say.

8431. eximius $L \in c$.

8432. pubicollis Lcc.

H33. eastor Lec.

8434. nigrirostris Fab.

\$435. quadricollis Lec.

-.. ? trivittatus Say.

Lepyrus Sch.

8436. gemellus Kirby.

8437. colon Linn.

\$438. geminatus Say.

Listronotus Jek.

8439. obliquus Lec.

8440. sordirus Gyll.

8441. tuberosus Lec.

8442. squamiger Say.

8443. callosus Ler.

8444. inæqualipennis Boh.

8445. caudatus Say.

8446. americanus Lec.

8447. rotundicollis Lec.

8448. appendiculatus $B o h$.

8419. sulcirostris Lec.

8450. nebulosus Lec.

8451. frontalis Lec.

8452. oregonensis Lec.

8453. latiusculus $B$ oh.

8454. cribricollis Lec.

8455. impressifrons Lec.

8456. setosus Lec.

\$457. punctiger Lec. 
8158. teretirostris Lec.

8459. gracilis Lec.

8160. nevadicus Lec.

\section{Macrops Kirby.}

8461. delumbis Gyll.

8462. lineatulus Say.

8463. sparsus Say.

8164. spurcus Boh.

8465. immundus $B$ oh.

8466. humilis Gyll.

8467. porcellus Say.

8468. vitticollis Kirby.

8469. solutus Boh.

\section{Emphyastes Mann.}

8470. fucicola Mann.

\section{Plinthus Germ.}

8471. carinatus $\boldsymbol{B}$ oh.

\section{Pissodes Germ.}

8472. strobi Peck.

8473. costatus Mann.

8474. fasciatus $L e c$.

8475. affinis Rand.

8476. dubius Rand.

8477. rotundatus Lec.

\section{Pachylobius Lec.}

8478. picivorus Germ. stupidus Boh.

\section{Hvlobius Germ.}

Q 8479. pales Hbst. assimilis $B o h$.

8180. confusus Kirby.

Hilipus Germ.

8481. squamosus $L e c$.

Hypomolyx Lec.

8482. pineti $F a b$. pinicola Coup.

\section{Eudocimus Sch.}

8483. Mannerheimii Boh.
Lixus Fab.

8484. pleuralis Lec.

8485. mixtus Lec.

S486. texanus Lec.

8487. rubellus Rand.

8488. auctus Lec.

8489. caudifor Lec.

8490. asper Lec.

8491. sylvius $B$ oh.

8492. fossus Lec.

8493. punctinasus Lec.

8494. pareus Lec.

8495. terminalis Lec.

8496. rectus $L e c$.

8497. mucidus Lec.

c. 8498. coneavus Say.

8499. musculus Say.

8500. perforatus Lec.

8501. scrobicollis $B$ oh.

8502. placidus Lec.

8503. læsicollis Lec.

8504. macer Lec.

8505. marginatus Say.

8506. præpotens Say.

8507. poricollis Mann.

8508. modestus Mann.

Centrocleonus Lee.

8509. pilosus Lec.

8510. angularis Lec.

8511. porosus Lec.

8512. molitor Lec.

Stephanocleonus Mots.

8513. plumbeus Lec.

8514. cristatus Lec.

Cleonopsis Lec.

8515. pulvereus Lec.

Cleonaspis Lec.

8516. lutulentus Lec.

Cleonus Sch.

8517. collaris Lec.

8518. trivittatus Say.

8519. inornatus Lec.

8520. frontalis Lec. 
8521. virgatus Lec.

8522. quadrilineatus Chev.

8523. canescens Lec.

8524. puberulus tec.

8525. carinicollis Lec.

8526. vittatus Kirby.

8527. calandroides Rand.

8528. sparsus Lec.

\section{Dorytomus Steph.}

C 8529. mucidus Say.

8530. laticollis Lec.

8531. rufulus Mann.

8532. brevicollis Lec.

8533. luridus Mann.

8534. subsignatus Mann.

8535. longulus Lec.

8536. squarnosus Lec.

8537. Mannerheimii Germ.

8538. hirtus Lec.

8539. hispidus Lec.

-_ lutulentus Sch.

- rufus Say.

_- rutilans $B o h$.

\section{Grypidius Seh.}

8540. equiseti $F a b$.

8541. brunnirostris Fab.

\section{Erycus Tourn.}

8542. morio Mann.

8543. puncticollis Lec.

\section{Procas Steph.}

8544. Lecontei Bedel. picipes $\ddagger L e c$.

\section{Desmoris Lec.}

C 8545. scapalis Lec.

C 8546. constrictus Say.

Barytychius Jek. Pachytychius $\ddagger$ Lec.

8547. amonus Say.

8548. discoideus Lec.

\section{Smicronyx Sch.}

8549. corpulentus Lec.

8550. ovipennis Lec.
8551. griseus Lec.

8552. obtectus Lec.

8553. flavicans Lec.

8554. pusio Lec.

8555. tychoides Lec.

8556. vestitus $L e c$.

8557. seriatus Lec.

C 8558. fulvus Lee.

8559. sordidus Lec.

8560. cinereus Mots.

8561. squamulatus Lec.

8562. corniculatus Fab.

Phyllotrox Sch.

8563. nubifer Lec.

8564. ferrugineus Lec.

Brachybamus Germ.

8565. electus Germ.

8566. inceratus $\mathrm{Boh}$.

\section{Onychylis Lec.}

8567. nigrirostris $B o h$.

8568. longulus Lec.

8569. alternans Lec.

\section{Endalus Lap.}

8570. setosus Lec.

8571. limatulus Gyll.

8572. æratus Lec.

8573. cribricollis Lec.

8574. punctatus Lec.

8575. ovalis Lec.

Tanysphyrus sch.

8576. lemnæ $F a b$.

Stenopelmus Sch.

8577. rufinasus Gyll.

Anchodemus Lec.

8578. angustus Lec.

8579. Hubbardi Lec.

8580. Schwarzi Lec.

\section{Lixellus Lec.}

8581. filiformis Lec. 
Lissorhoptrus Lec.

8582. simplex Siy.

$\$ 54:$. apiculatus Gyll.

\section{Bagous Germ.}

8584. mammillatus Say.

8585. sellatus Lec.

8586. planatus $L c c$.

8587. obliquus Lec.

8588. amerieanus Lec.

8589. cavifrons Lec.

8590. magister Lec.

8591. nebulosus Lec.

8592. californicus $L c c$.

8593. restrictus $L e c$.

8594. pusillus Lec.

8595. bituberusus Lec.

8596. transversus Lec.

_. binodulus Hbst.

\section{Pnigodes Lec.}

8597. setosus Lee.

\section{Phycocœtes Lee.}

8598. testacens Lec.

\section{Trachodes Germ.}

8599. ptinoides (jerm.

sio0. quadrituberculatus Mots.

S601. horridus Mann.

\section{Erodiscus Seh.}

8602. tinamus Lec.

\section{Otidocephalus Chev.}

8603. vittatus Horn.

stio4. Ulkei Horn.

8605. myrmex Hbst.

singh. serobienllis Boh.

sitio7. Chevrolatii Horn.

Stos. levicollis Horn.

8609. perforatus Horn.

S610. dichrous Lec.

\section{Magdalis Germ.}

8611. perforata IIorn.

8612. cuneiformis Horn.
5613. Lecontei Horn.

8614. imbellis Lec.

8615. barbita Say.

8616. ænescens Lec.

8617. olyra Hbst.

8618. gracilis Lec.

S619. subtincta Lec.

8620. hispoides Lec.

S621. gentilis Lee.

6022. sulicis Horn.

8623. inconspicua Horn.

8624. pandura Suy.

8625. armicollis Say.

Ch6626. pallida Say.

8627. alutacea Lec.

Acalyptus Sch.

s62s. carpini Hbst.

\section{Coccotorus Lec.}

8629. seutellaris Lfc.

\section{Anthonomus Germ.}

8630. quadrigibbus Say.

8631. nebulesus Lec.

8632. fulvus $L e c$.

s633. gularis Lec.

8634. profundus Lec.

8635. ater Lec.

8636. brunnipennis Mann.

Si37. scutellatus Gyll.

\$638. signatus Say.

S6:39. helvolus $B$ oh. rubidus Lec.

S640. juniperinus $S a n b$.

sit1. sycophanta Walsh.

S642. rufipeunis $L e c$.

S613. suturalis Lec.

86t4. musculus Sayy.

8645. suleifrons $L e c$.

sibth. flavicornis $B o h$.

8647. morulus Lec.

8645. nigrinus Boh.

8649. corvulus leec.

8650. pusillus $L e c$.

S651. elegans Lec.

C 8652. squnmosus Lec.

sisis. tectus $L c c$.

8654. hirtus LCC. 
8655. inermis $B$ oh.

8656. subvittatus Lec.

8657. pauperculus Lec.

8658. disjunctus Lec.

8659. rufipes Lec.

8660. elongatus Lec.

8661. eratægi Walsh.

8662. subfasciatus Lec.

S663. moleculus Casey.

8665. robustulus Lec.

8665. nubilus Lec.

8666. ungularis Lec.

8667. mixtus Lec.

8668. decipiens Lec.

8669. canus Lec.

8670. affinis Lec.

8671. nanus Jec.

-_ hæmatopus Boh.

\section{Orchestes IIl.}

8672. puberulus Boh.

8673. pallicornis Say.

8674. canus Horn.

8675. minutus Horn.

8676. rufipes Lcc.

8677. niger Horn.

8678. ephippiatus Say.

8679. subhirtus Horn.

8680. betuleti Horn.

\section{Macrorhoptus Lec.}

8681. estriatus Lec.

\section{Elleschus Steph.}

8682. bipunctatus Linn.

8683. ephippiatus Say. californicus Casey.

\section{Prionomerus Seh.}

8684. calceatus Say.

\section{Piazorhinus Sch.}

\$685. scutellaris Say.

S686. pictus Lec.

\section{Proctorus Lec.}

8687. armatus Lec.

\section{Encalus Lec.}

8688. decipiens Lec.
Thysanocnemis Lec.

Ch8689. fraxini Lec.

8690. helvolus Lec.

Plocetes Lec.

8691. ulmi Lec.

Tylopterus Lec.

8692. pallidus Lec.

8693. varius $L e c$.

Tychius Sch.

8694. arator Gyll.

8695. lineellus Lec.

8696. sordidus Lec.

8697. tectus Lec.

8698. semisquamosus Lec.

8699. hirtellus Lec.

8700. setosus Lec.

Bibynes Germ.

8701. fulvus Lec.

\section{Paragoges Lec.}

8702. maculatus Lec.

Nanophyes Sch.

C 8703. pallidulus Grav.

Cionus Clairv.

S704. scrophularice Linn.

Gymnetron Sch.

C 8705. teter Fab.

Miarus Steph.

8706. hispidulus Lec.

Nanus Seh.

8707. uniformis $B$ oh.

Notolomus Lec.

S708. ' bicolor Lec.

8709. myricse Lec.

8710 . basalis Lec.

Læemosaccus Sch.

8711. plagiatus Fab. 


\section{Conotrachelus Sch.}

8712. juglandis Lec.

8713. albicinctus Lec.

8714. nenuphar Hbst.

8715. retentus Say.

C 8716. seniculus Lec.

8717. affinis $B o h$.

8713. elegans Say.

8719. aratus Germ.

8720. nivosus Lec.

Ch 8721. cratægi Walsh.

8722. ventralis Lec.

8723. adspersus Lec.

5724. Belfragei Lec.

8725. similis Boh.

8726. naso Lec.

8727. posticatus $B o h$.

8728. cognatus Lec.

8729. pusillus Lec.

8730. geminatus Lec.

8731. infector Boh.

8732. cribricollis Say.

8733. coronatus Lec.

8734. tuberosus Lec.

8735. anaglypticus Say.

8736. leucophratus Fah.

8737. fissunguis Lec.

8738. erinaceus Lec.

8739. hispidus Lec.

-_ ? confinis Fah.

Micralcinus Lec.

8740. cribratus Lec.

Rhyssematus Chev.

8741. palmacollis Say.

8742. lineaticollis Say.

8743. æequalis Horn.

8744. pubescens Horn.

8745. pruinosus Boh.

\section{Chalcodermus Sch.}

8746. æneus Boh.

8747. inæquicollis Horn.

8748. collaris Horn.

8749. spinifer Boh.

\section{Zaglyptus Lee.}

8750. sulcatus Lec.

8751. striatus Lec.
Microhyus Lec.

8752. setiger Lec.

Acamptus Lec.

8753. rigidus $L e c$.

Eurhoptus Lec.

8754. pyriformis Lec.

Lembodes Sch.

8755. solitarius Boh.

Micromastus Lee.

8756. gracilis Boh.

Acalles Sch.

8757. Hubbardi Lec.

8758. nobilis Lec.

8759. basalis Lec.

8760. porosus Lec.

8761. turbidus Lec.

8762. clathratus Lec.

8763. carinatus Lec.

8764. costifer Lec.

8765. granosus Lec.

8766. subhispidus Lec.

8767. sordidus Lec.

8765. clavatus Say.

8769. crassulus Lec.

8770. Iongulus Lec.

8771. sulcicollis Lec.

8772. nuchalis Isec.

8773. pectoralis $L e c$.

8774. ventrosus $L e c$.

Pseudomus Sch.

8775. truncatus Lec.

8776. sedentarius Say.

8777. inflatus Lec.

\section{Tyloderma Say.}

5778. morbillosum Lec.

8779. foveolatum Say.

8780. variegatum Horn.

S7s1. fragariæ Riley.

8782. longum Lec.

8783. æreum Say. nigrum Casey. punctatum Casey.

8784. baridium Lec. 
Phyrdenus Lec.

8785. undatus Lec.

Cryptorhynchus Ill.

8786. parochus Hbst.

8787. bisignatus Say.

8788. pumilus $\boldsymbol{B o h}$.

8789. fuscatus $L e c$.

8790 . obliquus Say.

8691. helvus Lec.

8792. brachialis Lec.

8793. lutosus Lec.

8794. obtentus Hbst.

C 8795. fallax Lec.

8796. minutissimus Lec.

8797. apiculatus Gyll.

8798. tristis Lec.

8799. oblongus Lec.

8800. ferratus Say.

Macromerus Sch.

8801. mostus Lec.

\section{Zascelis Lec.}

8802. serripes Lec.

8803. squamigera Lec.

8804. irrorata Lec.

\section{Cœlosternus Sch.}

8805. hispidulus Lec.

\section{Baropsis Lec.}

8806. cribratus Lec.

\section{Zygops Sch.}

C 8807. seminiveus Lec.

\section{Piazurus Sch.}

8808. californicus Lec.

8809. oculatus Say.

8810. subfasciatus Lec.

\section{Copturus Sch.}

8811. operculatus Say.

8812. nanulus Lec.

8813. mammillatus Lec.

8814. adspersus Lec.
8815. quercus Say.

8816. longulus Lec.

8817. binotatus Lec.

8818. lunatus Lec.

8819. minutus Lec.

Acoptus Lec.

8820. suturalis Lec.

Tachygonus Sch.

8821. Lecontei Gyll.

8822. centralis Lec.

8823. tardipes Lec.

8824. fulvipes Lec.

Mononychus Germ.

8825. vulpeculus $F a b$.

Craponius Lec.

8826. inæqualis Say.

\section{Cnemogonus Lec.}

8827. epilobii Payk.

\section{Coliodes Sch.}

8828. curtus Say.

8829. tenuipes Lec.

8830. asper Lec.

8831. cruralis Lec.

8832. nebulosus Lec.

8833. nasalis Lec.

8834. flavicaudis Boh.

8835. acephalus Say.

Acallodes Lec.

Ch 8836. ventricosus Lec.

\section{Ceutorhynchus Germ.}

8837. subpubescens Lec.

8838. rapæ Gyll.

8839. sulcipennis Lec.

8840. decipiens Lec.

8541. rudis $L e c$.

8842. sericans Lec.

8843. convexicollis Lec.

8844. pusillus tec.

8845. pusio Mann.

8846. squamatus Lec. 


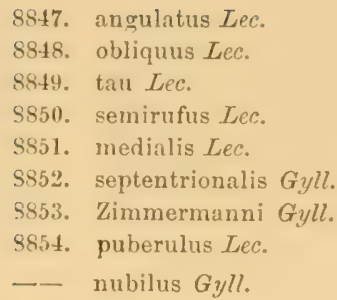

\section{Rhytidosomus Sch.}

8855. orobinus Schiödte.

\section{Phytobius Sch.}

8856. velatus Beck.

\section{Pelenomus Thom.}

8857. sulcicollis Fab.

8858. squamosus Lec.

8859. eavifrons $L e c$.

\section{Cœlogaster Sch.}

8860. Zimmermanni Gyll.

ch $886 \mathrm{l}$. cretura Hbst.

8862. obseurus Lec.

\section{Rhinoncus Sch.}

Ch 8863. pericarpius Linn. umbelle $F a b$.

C 8s6it. pyrrhopus Lec.

Ch S865. longulus Lec.

Orthoris Lec.

8s66. Crotehii Lec.

\section{Rhoptobaris Lec.}

8867. canescens Lec.

Baris Germ.

8868. strenua Lec.

8869. umbilicata Lce.

d 8870. striata Say.

8871. subovalis Lec.

8872. transversa Say.

8573. carinulata Lec.

8574. subrenea Lec.

8875. tumescens Lec.

8876. nitida Lec.
8877. interstitialis Say.

8878. confinis $L c c$.

8879. ærea Boh.

8880. sparsa Lec.

8881. macer Lec.

8s82. pruinosa Lec.

- californica Mots. conferta Boh.

\section{Trichobaris Lec.}

8883. texana Lec.

8884. trinotata Say. mucorea Lec.

8885. plumbea Lec.

\section{Pseudobaris Lec.}

C 8856. fareta Lec. 4887. pectoralis Lec. 88s8. nigrina Say. 88s9. anthracina Boh. 8890. angustula Lec. 8591. pusilla Lec. 8892. albilata $L c e$. 8893. t-signum Boh.

Onychobaris Lec.

8894. densa Lec. 8895. subtonsa Lec. 8896. pectorosa $\mathrm{Lec}$. 8s97. distans $L C c$. 8898. seriata Lec. 8899. eribrata Lec. 8900. rugicollis Lec.

Aulobaris Lee.

S901. scolopax Say. 8902. ibis Lec.

C 8903 . naso Lec.

Ampeloglypter Lee.

S904. sesostris Lucc.

S405. ater Lec.

890i. crenatus Lec.

Madarus Sch.

5907. undulatus Say.

\section{Eisonyx Lec.}

8908. crassipes Lec. 
Pachybaris Lec.

8909. porosus Lec.

Stethobaris Lec.

8910. tubulatus Say.

8911. corpulentus Lec.

Microcholus Lec.

8912. striatus Lec.

8913. puncticollis Lec.

8914. lævicollis Lec.

8915. erasus Lec.

\section{Calandrinus Lec.}

8916. grandicollis Lec.

\section{Centrinus Sch.}

8917. scutellum-album Say.
8918. penicellus Hbst.
? æratus Say.
8919. pistor Germ.
8920. dilectus Harr.
8921. lrevirostris Lec.
8922. punctirostris Lec.
8923. striatirostris Lec.
8924. modestus Boh.
8925. perscillus Gyll.
8926. neglectus Lec.
8927. eapillatus Lec.
8928. picumnus Hbst.
8929. perscitus Hbst.
8930. lineellus Lec.
8931. griseus Lec.
8932. decipiens Lec.
8933. lineicollis Lec.
8934. punctiger Lec.
8935. nasutus Lec.
8936. calvus Lec.
8937. rectirostris Lec.
8938. falsns Lec.
8939. canus Lec.

8940. longulus Lec.

8941. concinnus Lec.

8942. confusus Boh.

8943. prolixus Lec.

5944. confinis Lec.

8945. strigatus Lec.

\section{Zygobaris Lec.}

8946, nitens Lec.

8947. conspersa Lec.

8948. subealva Lec.

8949. convexa Lec.

\section{Barilepton Lec.}

8950. lutescens Lec.

8951. filiforme Lec.

8952. lineare Lec. albescens Lec.

8953. cribricolle Lec.

8954. quadricolle Lec.

8955. bivittatum Lec.

Euchætes Lec.

8956. echidna Lec.

Plocamus Lec.

8957. hispidulus Lec.

Hormops Lee.

8958. abducens Lec.

\section{Balaninus Germ.}

8959. obtusus Blanch.

8960. uniformis Lec.

8961. nasicus Say.

8962. caryæ Horn.

C 8963. rectus Say.

8964. quereus Horn.

8965. caryatrypes Boh.

__ porrectus Boh.

\section{BIRENTHIDE.}

\section{Cylas Lat.}

8966. formicarius $F a b$.

\section{Eupsalis Lec.}

8967. minuta Drury.

\section{Brenthus Fab.}

8968. peninsularis Horn.

8969. Iucanus Horn.

8970. snchorago Linn. 


\section{CALA N D I I D F.}

Rhynchophorus Hbst.

8971. cruentatus $F a b$.

8972. palmarum Linn.

\section{Scyphophorus Sch.}

8973. acupunctatus Gyll.

8974. robustior Horn.

8975. yuecre Horn.

Metamasius Horn.

S976. sericeus Lat.

\section{Cactophagus Lec.}

8977. validus Lec.

\section{Rhodobæenus Lec.}

C 8978. tredecimpunctatus Ill. 8979. pustulosus Gyll.

\section{Sphenophorus Sch.}

5980. simplex Lec.

C 8981. vomerinus Lec.

8982. Ulkei Horn.

C 8983. inrequalis Say.

8984, ochreus Lec.

8985. discolor Mann.

8986. pictus Lec.

\& 8987. pertinax Oliv.

8988. robustus Horn.

C 8989. costipennis Horn.

8990. cariosus Oliv.

8991. latinasus Horn.

8992. velutinus Lec.

C 8993. seulptilis Uhler.

8994. scoparius Horn.

C 8995. melanocephalus $F a b$.

8996. Sayi Gyll.

C 8997. apicalis Lec.

8998. placidus Say.

8999. gentilis Lec.

C 9000. parvulus Gyll.

9001. pumilus Gyll.

1) 9002. retusus Gyll.

9003. arizonensis Horn.

9004. gagatinus Gyll.

9005. cultellatus Horn.

9006. compressirostris Say.
9007. Germari Horn.

9008. variulosus Lec.

9009. oblitus Lec.

-_ fasciatus Fab.

- implicatus Sol.

- Lebasii Sch.

Trichischius Lec.

9010. crenatus Lec.

Calandra Clairv.

广) 9011. oryzœ Linn.

9012. remotepunctata Gyll

9013. granaria Linn.

\section{Yuccaborus Lec.}

9014. frontalis Lec.

Dryophthorus Sch.

7 9015. corticalis Say.

9016. bituberculatus $F a b$.

Dryotribus Horn.

9017. mimeticus Horn.

Lymantes Sch.

9018. scrobicollis Gyfl.

Gononotus Lec.

9019. lutosus Lec.

Himatium Woll.

9020. errans Lec.

9021. conicum Lec.

Cossonus Clairv.

9022. Bohemanni Horn.

9023. platalea Say.

9024. subareatus $B$ oh.

9025. piniphilus $B o h$.

9026. concinnus Boh.

9027. corticola Say.

9028. crenatus Horn.

9029. impressifrons Boh.

__ californicus Mots. 
Macrorhyncolus Woll.

9030. protractus Horn.

Macrancylus Lec.

9031. linearis Lec.

Allomimus Lec.

9032. dubius Horn.

Stenomimus Woll.

9033. pallidus Boh.

Caulophilus Woll.

9034. latinasus Say.

Mesites Sch.

9035. subcylindricus Horn.

9036. rufipennis Lec.

Phlœophagus Sch.

9037. apionides Horn.

9038. minor Horn.
Wollastonia Horn.

9039. quercicola Boh.

Amaurorhinus Fairm.

9040. nitens Horn.

Hexarthrum Woll.

9041. Ulkei Horn.

Elassoptes Horn.

9042. marinus Horn.

Rhyncholus Germ.

9043. oregonensis Horn.

9044. brunneus Mann.

9045. dorsalis Lec.

9046. angularis Lec.

9047. corticalis Boh.

Stenoscelis Woll.

9048. brevis Boh.
Platypus Hbst.

9049. flavicornis $F a b$.

9050. quadridentatus Oliv.

9051. compositus Say.

9052, rugulosus Chap.

9053. punctulatus Chap.

\section{Corthylus Er.}

9054. punctatissimus Zimm.

Monarthrum Kirsch.

9055. fasciatum Say.

9056. sentellare Lec.

9057. dentigerum Lec.

9058. mali Fitch.

\section{Gnathotrichus Eich.}

\section{Pityophthorus Eich.}

9059. retusus Lec.

9060. materiarius Fitch.

9061. asperulus Lec.

\section{SCOLYTIDA.}

9062: minutissimus Zimm.

9063. pubipennis Lec.

9064. pilosulus Lec.

9065. carinulatus Lec. hamatus Lec.

9066. pullus Zimm.

9067. plagiatus Lec.

9068. sparsus Lec.

9069. pulchellus Eich.

9070. pulicarius Zimm.

9071. eariniceps Lec.

9072. fossifrons $L e c$.

9073. confinis Lec.

9074. nitidulus Mann.

9075. annectens Lec.

9076. consimilis Lec.

9077. hirticeps Lec.

9078. deletus Lec.

9079. puncticollis Lec.

9080. pusio Lec.

9081. lautus Eich.

9082. puberulus Lec.

9083. digestus Lec.

9084. obliquus Liec. 
9085. seriatus Lec.

9086. comatus Zimm.

9087. opaculus Lec.

\section{Hypothenemus Westw.}

9088. eruditus Westw. hispidulus Lec.

9089. erectus Lec.

9090. dissimilis Zimm.

9091. striatus Lec.

_- asperulus Eich.

\section{Xyloterus Er.}

9092. retusus Lec.

9093. bivittatus Kirby.

9094. scabricollis Lec.

9095. politus Say.

9096. unicolor Eich.

\section{Cryphalus Er.}

9097. rigidus Lec.

9098. miles Lec.

9099. striatulus Mann.

9100. robustus Eich.

9101. mucronatus Lec.

__ terminalis Mann.

Coccotrypes Eich.

9102. jalappæ Letz.

\section{Xyleborus Eich.}

9103. tachygraphus Zimm.

9104. pyri Peck.

9105. obesus Lec.

9106. celsus Eich.

9107. fuseatus Eich.

9108. biographus Lec.

9109. retusicollis Zimm.

9110. xylographus Say.

9111. pubescens Zimm.

6 9112. cælatus Eich.

9113. impressus Eich.

9114. punctipennis Lec.

9115. planicollis Zimm.

_- propinquus Eich.

_-_ decretus Eich.
Dryocœtes Eich.

9116. septentrionis Mann.

9117. aftaber Mann.

9118. granicollis Lec.

\section{Xylocleptes Ferran.}

9119. concinnus Mann.

9120. decipiens Lec.

9121. cucurbitæ Lec.

\section{Tomicus Lat.}

9122. calligraphus Germ.

9123. cacographus Lec.

9124. confusus Lec.

9125. plastographus $L e c$.

Ef 9126. emarginatus Lec.

9127. rectus Lec.

9128. pini Say.

9129. hudsonicus Lec.

9130. interruptus Mann.

9131. mucronatus Lec.

9132. tridens Mann.

9133. avulsus Eich.

9134. latidens Lec.

9135. balsameus Lec.

- hirsutus Eich.

--- oregonus Eich.

- perturbatus Eich.

_._. terminatus Mann.

Micracis Lee.

9136. suturalis $L c c$.

9137. aculeata Lec.

9138. nanula $L e c$.

9139. asperula Lec.

9140. opacicollis Lec.

9141. rudis Lee.

9142. hirtella Lec.

\section{Thysanœs Lec.}

9143. fimbricornis Lec.

\section{Scolytus Geoff.}

9144. quadrispinosus Say.

9145. fagi Walsh.

9146. unispinosus Lec. 
9147. ealifornicus Lec.

9148. muticus Say.

9149. præceps Lec.

9150. subscaber Lec.

9151. sulcatus Lec.

9152. ventralis Lec.

9153. rugulosus Ratz.

Chramesus Lec.

9154. icoriæ Lec. Lecontei Chap.

9155. Chapuisii Lec.

\section{Rhopalopleurus Chap.}

9156. pumilus Chap.

\section{Polygraphus Er.}

9157. rufipennis Kirby.

9158. brevicornis Kirby.

\section{Phlœotribus Lat.}

9159. liminaris Harr. 9160. frontalis Oliv.

9161. puberulus Lec.

\section{Hylesinus Fab.}

9162. imperialis Lec.

9163. aculeatus Say.

9164. fasciatus $L e c$.

9165. sericeus Mann.

9166. opaculus Lec.

9167. aspericollis Lec.

\section{Cnesinus Lec.}

9168. strigicollis Lec.

Bothrosternus Eich.

\section{Phlœosinus Chap.}

9169. serratus Lec.

9170. cristatus Lec.

9171. dentatus Say.

9172. punctatus Lec.

9173. Haagii Eich.

9174. graniger Chap.
Chætophlœus Lec.

9175. hystrix Lec.

\section{Carphoborus Eich.}

9176. simplex Lec.

9177. bifurcus Eich.

9178. bicristatus Chap.

Dendroctonus Er.

Y 9179. terebrans Oliv.

9180. similis Lec.

9181. rufipennis Kirby.

9182. punctatus Lec.

9183. simplex Lec.

9184. brevicomis Lec.

9185. frontalis Zimm.

Crypturgus Er.

$\Delta$ 9186. atomus Lec.

Dolurgus Eich.

9187. pumilus Mann.

Scierus Lec.

9188. annectens Lec.

Hylastes Er.

9189. Inacer Lec.

9190. longus Lec.

9191. nigrinus Mann.

9192. porculus $E$.

9193. eavernosus Zimm.

\& 9194. gracilis Lec.

9195. porosus Lec.

9196. tenuis Zimm.

9197. exilis Chap.

Hylurgops Lee.

* 9198. granulatus Lec.

† 9199. pinifex Fitch.

9200. rugipennis Mann.

9201. subcostulatus Mann.

-— ? rufipes Eich.

-_ ? cristatus Mann. 


\section{N'THARIIBI D E.}

Ischnocerus Seh.

9202. infuscatus $F a b$.

Gonotropis Lec.

9203. gibbosus Lec.

Eurymycter Lec.

9204. fasciatus Oliv.

Tropideres Sch.

9205. bimaculatus Oliv.

9206. rectus Lec.

Allandrus Lec.

9207. bifasciatus Lec.

Hormiscus Waterh.

9208. saltator Lec.

Toxotropis Lec.

9209. pusillus Lec.

9210. fasciatus Lec.

9211. approximatus Lec.

Gonops Lec.

9212. fissunguis Lec.

Eusphyrus Lec.

9213. Walshii Lec.

Phcenicobius Lec.

9214. chameropis Lec.

Piezocorynus Sch.

9215. dispar Gyll.

9216. mixtus Lec.

9217. mostus Lec.
Anthribus Geoff.

9218. cornutus Say.

9219. lividus Lec.

Toxonotus Lac.

9220. fascicularis Sch.

Cratoparis Sch.

$>$ 9221. lunatus Fab.

9222. lugubris Oliv.

Brachytarsus Sch.

9223. alternatus Say.

9224. griseus Lec.

9225. limbatus Say.

9226. plumbeus Lec.

9227. vestitus $L e c$.

9228. tomentosus Say. paululus Casey.

9229. variegatus Say.

Anthribulus Lec.

9230. rotundatus Lec.

Aræocerus Sch.

9231. fasciculatus $D e G$.

Choragus Kirby.

9232. Zimmermanni Lec.

9233. Sayi Lec.

9234. Harrisii Lec.

9235. nitens Lec.

Xenorchestes Woll.

9236. americanus Mots.

Euxenus Lee.

9237. punctatus Lec.

9238. piceus Lec. 


\section{INDEX.}

Ababactus, 38.

Abrreus, 57.

Acalles, 142.

Acallodes, 143.

Acalyptus, 140.

Acamptus, 142.

Acanthocerus, 89.

Acanthocinus, 103.

Acanthoderes, 102.

Achrastenus, 135.

Achryson, 95.

Acidota, 42.

Acilius, 22.

Acmægenius, 136.

Acmæodera, 74.

Acmæops, 100.

Acneus, 64.

Acolpus, 54.

Acoptus, 143.

Acratus, 89.

Acritus, 57.

Acrulia, 43.

Actenodes, 74.

Actidium, 44 .

Actobius, 34.

Acupalpus, 17.

Acylophorus, 33.

Adalia, 47.

Adelina, 119.

Arlelocera, 65.

Adelops, 27.

Adelothyreus, 65.

Adimonia, 111.

Adonia, 46.

Adoxus, 108.

Adranes, 28.

Egialia, 87.

Agialites, 122.

Egialitide, 122.

Eletes, 57.

Ethecerus, 98.

Agabetes, 21.

Agabinus, 21.

Agabus, 21.

Agallissus, 99.

Agaosoma, 16.

Agasphærops, 133.

Agathidium, 27.

Agelastica, 110.

Aglenus, 51.

Aglyptus, 28 .
Agonoderus, 16. Agraphus, 134.

Agrilus, 75.

Agriotes, 68 .

Agronus, 135.

Agrypnus, 65 .

Agyrtes, 26.

Alæephus, 119.

Alaudes, 120.

Alaus, 65.

Aleochara, 32.

Alexia, 49.

Alindria, 60 .

Allandrus, 150.

Allecula, 122.

Allomimus, 147.

Allonyx, 81.

Allopoda, 125.

Allopogon, 63 .

Allorhina, 93.

Alobus, 91.

Alphitobius, 120.

Amannus, 98.

Amara, 9.

Amartus, 57.

Amaurorhinus, 147.

Amblychila, 1.

Amblyctis, 124.

Amerizus, 5.

Ammodonus, 120.

Amnesia, 134.

Ampeloglypter, 144.

Amphasia, 18.

Amphicerus, 85.

Amphichroum, 42.

Amphicoma, 89.

Amphicrossus, 58.

Amphicyrta, 61 .

Amphidora, 118.

Amphionycha, 104.

Amphizoa, 18.

Aмphizolde, 18.

Anachilus, 71.

Anacyptus, 39.

Anæedus, 121.

Anamesus, 71.

Anametis, 134.

Anamorphus, 49.

Anapleus, 56 .

Anaspis, 126.

Anatis, 47.
Anatrichis, 16.

Ancous, 43.

Anchastus, 66 .

Anchodemus, 139.

Anchomma, 50.

Anchonoderus, 12.

Anchycteis, 63.

Anchytarsus, 63.

Ancylocera, 97.

Ancyronyx, 63.

Ancyrophorus, 42.

Andrector, 110.

Androchirus, 123.

Androlyperus 110.

Aneflus, 96.

Anelastes, 65 .

Anemia, 121.

Anepsius, 116.

Anillus, 7.

Anisocalvia, 47.

Anisodactylus, 18.

Anisomera, 21.

Anisosticta, 46.

Anisotarsus, 18.

Anisotoma, 27.

Anisoxya, 124 .

Anobium, 83.

Anogdus, 27.

Anomadus, $13 \%$.

Anomala, 92.

Anomca, 105.

Anomoglossus, 15.

Anophthalmus, 8.

Anorus, 63.

Anthaxia, 73.

Antherophagus, 52.

ANтнисіD $\approx, 128$.

Anthicus, 129.

Anthobium, 43.

Anthocomus, 80 .

Anthonæus, 57.

Anthonomus, 140.

Anthophilax, 100.

Anthrenus, 54.

ANThribin e, 150.

Anthribulus, 150 .

Anthribus, 150.

Apenes, 14.

Aphanotus, 121 .

Aphelogenia, 13.

Aphodius, 88. 
Aphonus, 93.

Aphorista, 49.

Aphrastus, 135.

Aphricus, 71.

Aphthona, 113.

Apion, 136.

Aplastus, 71.

Apocellus, 42.

Apocrypha, 122.

Apristus, 13.

Apsectus, 54.

Aptopus, 66.

Aracanthus, 136.

Arseocerus. 150.

Aræopus, 63.

Arreoschizus, 116.

Aragnomus, 135.

Aramigus, 135.

Ardistomis, 5 .

Areus, 35.

Argopistes, 113.

Argoporis, 118.

Arhopalus, 99.

Arpedium, 42.

Arthmius, 29.

Arthrolips, 46.

Arthromacra, 123.

Artipus, 135.

Asaphes, 71.

Asclera, 126.

Asemum, 94.

Asida, 117.

Aspidoglossa, 5.

Astrotus, 117.

Atronius, 87.

Ataxia, 103.

Atemeles, 31.

Athous, 69.

Atimia, 99.

Atinus, 29.

Atomaria, 53.

Atranus, 12.

Attagenus, 54.

Attitlus, 80,

Attelabid.

Attelabus, 133.

Auchmobius, 115.

Auletes, 133.

Aulicus, 81 .

Aulobaris, 144.

Aulonium, 50.

Autalia, 32.

Axestinus, 96.

Axinocerus, 54.

Axinopalpus, 14.

Babia, 106.

Bacanius, 57.

Bactridium, 61.

Bactrocerus, 128.

Badister, 11.

Bæocera, 45.
Bagous, 140.

Balannius, 145.

Baptolinus, 35 .

Barilepton, 145 .

Baris, 144.

Baropsis, 143.

Barynotus, 133.

Barytychius, 139.

Bassareus, 106.

Batrisus, 29.

Batulius, 116.

Batyle, 98.

Belionota, 74.

Bellamira, 100.

Belonuchus, 33 .

Bembidium, 5.

Berginus, 53.

Berosus, 24.

Betarmon, 68.

Bidessus, 19.

Bitoma, 85.

Bius, 119.

Bladus, 70.

Blaps, 118.

Blapstinus, 119.

Blauta, 67.

Blechrus, 13.

Bledius, 41.

Blepharida, 111.

Blethisa, 3.

Bolboceras, 89 .

Boletobius, 40.

Boletophagus, 122.

Boletotherus, 122.

Bolitochara, 32.

Bonvouloiria, 59.

Boros, 125.

Bostrychus, 85.

Bothrideres, 51.

Bothriophorus, 62.

Bothrosternus, 149.

Brachyacantha, 47.

Brachybaruus, 139.

Brachyderus, 133.

Brachylobus, 15.

Brachynus, 15.

Brachypeplus, 58.

Brachypsectra, 63.

Brachypterus, 57.

Brachys, 75 .

Brachystylus, 135.

Brachytarsus, 150.

Bradycellus, 17.

Bradycinetus, 8.8 .

Branchus, 117.

Brathinus, 28.

BRENTHID.E, 145.

Brenthus, 145.

Brontes, 52.

Brothylus, 96.

BRUCHID E, 114.

Bruchus, 114.
Bryaxis, 29.

Brychius, 18.

Bryoporus, 40.

BupIESTID.F. 72.

Buprestis, 73.

BYRRHID.E, 61.

Byrrhodes, 84.

Byrrhus, 61.

BYRsopid £, 133.

Bythinus, 29.

Byturus, 54.

Cacoplia, 102.

Cactophagus, 146.

Cænia, 76 .

Cænocara, 84.

Cæeporis, 112.

Cafius, 35.

Calandra, 146.

Calandrid a, 146.

Calandrinus, 145.

Calathus, 11.

Calitys, 61.

Callicerus, 31 .

Callichroma, 97.

Callida, 14.

Callidium, 95.

Callimoxys, 97.

Callimus, 97.

Callimus, 97.

Calloides, 98.

Calochromus, 76 .

Calodera, 31.

Calopteron, 75.

Calopus, 125.

Calosoma, 3.

Calospasta, 132.

Caly ptillus, 134.

Culyptocephalus, 76 .

Calyptomerus, 28.

Campylus, 69.

Canifi, 125.

Cantharis, 131.

Canthon, 86.

Canthydrus, 19.

Capuochroa, 123.

Carabidar, 2.

Carabus, 3.

Cardiophorus, 66.

Carebara, 124.

Carphoborus, 149.

Carpophilus, 57.

Caryoborus, 114.

Casnonia, 12.

Cassida, 114.

Catogenus, 51 .

Catoptrichus, 26.

Catorama, 84.

Caulophilus, 147.

Cebrio, 71.

Cedius, 29.

Celetes, 76 . 
Celina, 19.

Cenophengus, 77.

Centrinus, 145.

Centrioptera, 116.

Centrocleonus, 138.

Centrodera, 100.

Centronopus, 119.

Ceophyllus, 29.

Cepraloid f, 126.

Cephaloon, 126.

Cephaloscymnus, 48.

Cephennium, 28.

Ceracis, 86.

Cerambycid a, 94.

Cerataltica, 112.

Ceratomegilla, 46.

Cercocerus, 29.

Cercopeus, 135.

Cercus, 57.

Cercyon, 25.

Cerenopus, 118.

Cerophytum, 72.

Cerotoma, 110.

Ceruchus, 86.

Cerylon, 51.

Ceutorhynchus, 143.

Chrtarthria, 24.

Chretechus, 135.

Chrtocnema, 113.

Chætocœlus, 79.

Chrtophlous, 149.

Chalcodermus, 142.

Chalcolepidius, 65 .

Chalcophora. 72.

Chalepus, 92.

Chariessa, 82.

Charistena, 114.

Chauliognathus, 77.

Chelonarium, 62.

Chelymorpha, 114.

Chevrolatia, 28.

Chilocorus, 47.

Chilometopon, 116.

Chion, 96.

Chlænius, 15.

Chlamys, 106.

Chnaunanthus, 89.

Chœridium, 87.

Cholerus, 28.

Choleva, 26.

Choragus, 150.

Chramesus, 149.

Chromatia, 123.

Chrysobotbris, 73.

Chrysochus, 108.

Chrysodina, 109.

Chrysomela, 109.

Chrisomelid

Chrysophana, 74.

Cibdelis, 119.

Cicindela, 1.

Cicindelide, 1.
Cicones, 50.

Cilea, 40.

Cimbocera, 134.

Cinyra, 73.

CIOID A, 85.

Cionus, 141.

Cis. 85.

Cistela, 123.

Cistelid A, 122.

Clambus, 28.

Cleonaspis, 138.

Cleonopsis, 138.

Cleonus, 138.

Clerid e, 81.

Cleronomus, 81.

Clerus, 81.

Clinidium, 51.

Clivina, 5.

Cløotus, 89.

Clytanthus, 99.

Clytus, 99.

Cnemeplatia, 120.

Cnemidotus. 19.

Cnemodus, 116.

Cnemogonus, 143.

Cnesinus, 149.

Coccidula, 48.

Coccinella, 47.

Coccinelid E, 46 .

Coccotorus, 140.

Coccotrypes, 148.

Colambus, 19.

Coeliodes, 143.

Colocnemis, 119.

Cologaster, 144.

Coelosternus, 143.

Coelotaxis, 117.

Cœlus, 117.

Ccenonycha, 90.

Conopous, 102.

Coenoscelis, 53.

Colaspis, 109.

Colastus, 57.

Colenis, 27.

Coleocerus, 136.

Collops, 79.

Colon, 27.

Colpius, 19.

COLYDIIDE, 50.

Colydium, 51.

Colymbetes, 22.

Compsa, 96.

Compsus, 135.

Conibius, 120.

Coninomus, 59.

Coniontis, 117.

Conoecus, 115.

Cononotus, 125.

Conosoma, 40.

Conotelus, 58.

Conotrachelus, 142.

Copelatus, 21.
Copidita, 126.

Copris, 87.

Coprophilus, 42.

Coptocycla, 114.

Coptodera, 13.

Coptostethus, 66.

Coptotomus, 21.

Copturus, 143.

Cordylospasta, 130.

Corphyra, 128.

Corthylus, 147.

Corticaria, 59.

Corylophide, 46.

Corylophus, 46 .

Corymbites, 70 .

Coscinoptera, 106.

Cossonus, 146.

Cotalpa, 92.

Cnxelus, 50.

Craniotus, 115.

Craponius, 143.

Cratacanthus, 16.

Cratidus, 118.

Cratoparis, 150.

Cregya, 82.

Cremastochilus, 93.

Creophilus, 33.

Crepidodera, 112.

Crigmus, 68.

Criocephalus, 94.

Crioceris, 105.

Crioprosopus, 97.

Crossidius, 98.

Crymodes, 125.

Cryphalus, 148.

Cryptarcha, 59.

Crypticus, 120.

Cryptobium, 37.

Cryptocephalus, 106.

Cryptoglossa, 116.

Cryptognatha, 47.

Cryptohypnus, 66.

Cryptophagid. 52.

Cryptophagus, 53.

Cryptopleurum, 25.

Cryptorhopalum, 54.

Cryptorh ynchus, 143.

Cryptostoma, 65.

Crypturgus, 149.

Cteniopus, 123.

Ctenistes, 29.

Ctenobium, 83.

CucuJid 251.

Cucujus, 52.

Cupes, 85.

CUPRSID $\approx, 85$.

Curculionid 玉, 136.

Curius, 97.

Cybister, 23.

Cybocephalus, 59.

Cychramus, 59.

Cychrus, 2. 
Cyclocephala, 92 .

Cycloneda, 47.

Cyclonotum, 25.

Cylas, 145.

Cyllene, 98.

Cyllodes, 59.

Cymatodera, 81.

Cymatopterus, 22.

Cymindis, 14.

Cynzus, 120.

Cyparium, 45.

Cypherotylus, 50 .

Cyphomimus, 136.

Cyphon, 64.

Cyphus, 135.

Cyrtinus, 102.

Cyrtophorus, 99.

Cyrtotriplax, 50.

Cyrtusa, 27.

Cysteodemus, 130.

Cytilus, 61.

Dacne, 49.

Dacnochilus, 38.

Dacoderus, 116.

Daetylosternum, 25.

Dascrllide, 63.

Daseyllus, 63.

Dasycerus, 59 .

Dasydera, 89.

Dasyglossa, 32 .

Dasytes, 81.

Dearthrus, 54.

Decarthron, 29.

Dectes, 103.

Deleaster, 42.

Deliphrum, 42.

Deltochilum, 87.

Deltometopus, 64 .

Dendrobius, 97

Dendrocharis, 64.

Dendroctonus, 149.

Dendroides, 129.

Deadrophagus, 52.

Dendrophilus, 56 .

Derallus, 24.

Deretaphrus, 51 .

Dermestes, 54.

DERMESTIDE, 54 .

Derobrachus, 94.

DERODONTID.E, 61.

Derodontus, 61.

Deronectes, 20.

Desmiphora, 103.

Desmocerus. 99.

Desniopachria, 19.

Desmoris, 139.

Diabrotica, 110.

Diachila, 3.

Diachus, 10 7.

Dialytes, 87 .

Diamimus, 134.
Dianchomene, 13.

Dianous, 35.

Diaperis, 121.

Diaphorus, 13.

Diazus, 90.

Dibolia, 113.

Dicælus, 10.

Dicentrus, 95.

Dicerca, 72.

Dichelonycha, 90.

Dichoxenus, 134.

Diclidia, 126.

Dicranopselaphus, 63 .

Dicrepidius, 67.

Dictyoptera, 76 .

Dignamptus, 122.

Dineutes, 23.

Dinoderus, 85 .

Dinopsis, 32.

Diochus, 35 .

Diodyrhynchus, 133.

Dicedus, 120.

Diplochila, 10.

Diplocolus, 53.

Diplotaxis, 90.

Dircrea, 124.

Dirotognathus, 135.

Discoderus, 16.

Discogenia, 118.

Disonycha, 111.

Distenia, 99.

Ditemnus, 79.

Ditoma, 50.

Ditylus, 125.

Dolichosoma, 80 .

Dolopius, 68.

Dolurgus, 149

Donacia, 104.

Dorcaschema, 102.

Dorcasta, 103.

Dorcatoma, 84.

Dorcus, 86.

Doryphora, $10 y$.

Dorytomus, 139.

Drapetes, 72.

Drasterius, 68.

Dromæolus, 64 .

Dromius, 13.

Dryobius, 95.

Dryocœetes, 148.

Dryophthorus, 146.

Dryops, 62.

Dryotribus, 146.

Dularius, 95.

Dynastes, 93.

Dyschirius, 4.

Dyscinetus, 92.

Dyslobus, 134.

Dysmerus, 52.

Dysphaga, 104.

Dystaxia, 74 .

Dysticheus, 135.
I DYTISCIDE, 19.

Dytiscus, 22 .

Eburia, 96.

Echidnoglossa, 31.

Echinocoleus, 26.

Echinodes, 55.

Echocerus, 120.

Ectopria, 63.

Acyrus, 103.

Edaphus, 37.

Edrotes, 115.

Ega, 13.

Eisonyx, 144.

Elaphidion, 96.

Elaphrus, 3.

Elasmocerus, 81 .

Elassoptes, 147.

Elater; 67.

Elateride, 64.

Elateropsis. 94.

Elatrinus, 68.

Elendes, 118.

Eleusis, 44.

Elleschus, 141.

Ellyclinia, 76 .

Elmis, 62.

Elytroleptus, 97.

Embaphion, 118.

Emmenastus, 116.

Emmesa, 124.

Empelus, 28.

Emphyastes, 138.

Emphylus, 53.

Emplenota, 31.

Encalus, 141.

Enchodes, 124.

Encyclops, 100.

Endalus, 139.

Endecatomus, 84.

Endendes, 79.

EхромхсніD Е, 49.

Endomychus, 49 .

Endoplilous, 50.

Euiconyx, 71.

Ennearthron, 86.

Enoplium, 82.

Entumophthalmus, 65.

Entomoscelis, 109.

Entomnsterna, 98.

Ephalus, 120.

Ephelis, 43.

Ephistemus, 53.

Epicerus, 133.

Enicauta, 131.

Epierus, 55.

Epilachna, 48.

Epimetopus, 24.

Fpiphanis, 65.

Epipocus, 49.

Epitragus, 116.

Epitrix, 112. 
Epurrea, 58.

Erchomus, 40.

Eretes, 22.

Ergates, 94.

Eriopis, 46.

Ernobius, 83.

Erodiscus, 140.

Eros, 76.

Erotylathris, 51.

EROTYLID.E, 49.

Erotylus, 50.

Erycus, 139.

Eschatocrepis, 80.

Esthesopus, 66.

Estola, 103.

Euæsthetus, 37.

Eucærus, 14.

Euceratocerus, 84.

Euchætes, 145.

Eucinetus, 63.

Eucrada, 83.

Eucrossus, 95.

Eucyllus, 135.

Eudectus, 43.

Eudera, 32.

Euderces, 99.

Eudesma, 50.

Eudiagogus, 136.

Eudocimus, 138.

Eugnamptus, 133.

Eulabis, 118.

Eumichthus, 97.

Eumicrus, 28.

Eupactus, 84.

Eupagoderes, 134.

Euparia, 87.

Euphoria, 93.

Euphorticus, 12.

Euplectroscelis, 113.

Euplectus, 30 .

Eupogonius, 103.

Eupompha, 132.

Eupristocerus, 75.

Euproctus, 14.

Eupsalis, 145.

Eupsenius, 30.

Eupsophus, 119.

Eurhoptus, 142.

Europs, 61.

Eurygenius, 128.

Eurymetopon. 115.

Eurymyeter, 150.

Eurypogon, 63.

Euryporus, 33.

Euryptera, 101.

Euryscopa, 105.

Eurysphindus, 86.

Euryusa, 32.

Eusattus, 117.

Eusphyrus, 150.

Eustroma, 96.

Eustrophus, 124.
Eutheia, 28.

Euthorax, 32.

Euthysanius, 71 .

Eutochia, 121.

Eutrichites, 30.

Eutyphlus, 30.

Euxenus, 150.

Evarthrus, 9.

Evolenes, 16.

Evoplus, 120.

Evotus, 136.

Exema, 106.

Exochomus, 47.

Falagria, 31 .

Faliscus, 30 .

Faronus, 30 .

Fidia, 108.

Formicomus, 129.

Fornax, 64 .

Fustiger, 29.

Galerita, 12.

Galeruca, 111.

Galerucella, 111.

Ganimus, 95.

Gastrallus, 83.

Gastroidea, 109

Gaurodytes, 21.

Gaurotes, 100.

Geoderces, 135.

Geodromicus, 42.

Geopinus, 16.

Georysid E, 62.

Georyssus, 62.

Geotrupes, 89.

Gibbium, 83.

Glaresis, 89.

Glıpa, 126.

Glipodes. 127.

Glycia, 14.

Glycobius, 98.

Glyphonyx, 68 .

Glyptina, 113.

Glyptoma, 43.

Glyptoscelis, 108.

Glyptotus, 119.

Gnaphalodes, 96.

Gnathium, 130.

Gnathocerus, 120.

Gnathospasta, 131.

Gnathotrichus, 147.

Gnorimus, 93.

Goes, 102.

Gonjoctena, 110.

Gonocallus, 95.

Gononotus, 146.

Gonops, 150 .

Gonotropis, 150 .

Gracilia, 95.

Graphisurus, 103.

Graphoderes, 23.
Graphops, 108.

Graphorhinus, 133.

Graptodera, 112.

Griburius, 107.

Grynocharis, 61 .

Grypidius, 139.

Gyaseutus, 72.

Gymnetis, 93.

Gymnetron, 141 .

Gymnusa, 32.

Gynandropus, 16.

Gynandrotarsus, 18.

Gyretes, 23.

GYRINID E, 23.

Gyrinus, 23.

Gyrohypnus, 35.

Gyrophæna, 32.

Habrocerus, 40.

Hadrobregmus, 83.

Hadromerus, 135.

Hadrotes, 33.

Hæmonia, 105.

Halecia, 72.

HALIPLID E, 18.

Haliplus, 18.

Hallomenus, 124.

Haltici, 112.

Hamletia, 111.

Hammaticherus, 96.

Haplandrus, 119.

Haplidus, 95.

Haploderus, 41.

Haploglossa, 32.

Harmonia, 47.

Harpalus, 16.

Hedobia, 83.

Helichus, 62.

Helluomorpha, 14.

Helodes, 64.

Helopeltis, 25.

Helophorus, 23.

Helops, 122.

Hemipeplus, 52.

Hemiptychus, 84.

Hemirhipus, 65.

Henoticus, 53.

Henous, 130.

Hesperobænus, 61 .

Hetærius, 55.

Heterach thes, 96.

HETEROCERID.e, 63.

Heterocerus, 63.

Heterothops, 33.

Hetœmis, 102.

Hexarthrum, 147.

Hilipus, 138.

Himatium, 146.

Hippodamia, 46.

Hippomelas, 72.

Hippopsis, 103.

Hister, 55. 
Histeride, 54.

Holciophorus, 8.

Hololepta, 54.

Holoparamecus, 59.

Holopleura, 97.

Holotrochus, 41.

Homæsthesis, 94.

Homatium, 43.

Homalota, 31.

Homøusa, 32.

Hoplandria, 31 .

Hoplia, 89.

Hoplocephala, 121.

Hoplosia, 103.

Horia, 130.

Horistonotus, 66.

Hormiscus, 150 .

Hormops, 145.

Hormorus, 133.

Hornia, 130.

Hybodera, y7.

Hybosorus, 88.

Hydaticus, 22.

Hydnobius, 27.

Hydnocera, 82.

Hydræna, 24.

Hydrobius, 25.

Hydrocanthus, 19.

Hydrocharis, 24.

Hydrochus, 24.

Hydrocombus, 25.

HYDROPHILIDE, 23.

Hydrophilus, 24.

Hydroporus, 20.

Hydroscapha, 45 .

HYDROSCAPHID.E, 45.

Hydrotrupes, 21.

Hydrovatus, 19.

Hylastes, 149 .

Hylecoetus, 85 .

Hylesinus, 149.

Hylobius, 138.

Hylochares, 65.

Hylotrupes, 95 .

Hylurgops, 149.

Hymenorus, 123.

Hyperaspidius, 48.

Hyperaspis, 48.

Hyperplatys, 103.

Hypexilis, 95.

Hypocoelus, 65.

Hy pocoprus, 53.

Hypocyptus, 39 .

Hypodacne, 49.

Hypolampss, 111.

Hy pomolyx, 138.

Hy pophlous, 121.

Hy porhagus, 123.

Hypotelus, 44.

Hy pothenemus, 148

Hypotrichia, 90.

Hypulus, 124.
Ichnea, 82.

Idœmea, 104.

Ilybiosoma, 21.

Ilybius, 21 .

Ilyobates, 31 .

Ino, 52 .

Iphthimus, 119.

[pochus, 101.

Ips, 59.

Ischalia, 129.

Ischiodontus, 67.

Ischnocerus, 150.

Ischnocnemis, 98 .

Ischyrus, 50 .

Isomira, 123.

Isoplastus, 27.

Ithycerus, 136.

Kalissus, 44.

Laccobius, 24.

Lacconotus, 125.

Laccophilus, 19.

Lachnocrepis, 15.

Lachnophorus, 12.

Lachnopus, 136.

Lachnosterna, 91.

Lacon, 95.

Lactica, 112.

Læmophlœus, 52.

Læmosaccus, 141.

Lagochirus, 102.

LAGRIIDE, 123.

Lamprohiza, 77.

LAMPYRID.E, 75 .

Languria, 49.

Langurites, 49.

Lara, 62.

Laricobius, 82.

Lasconotus, 50.

Lasioderma, 84.

Lathrimæum, 42 .

Lathrobium, 38.

Lathropus, 52.

LATRIDIID.e, 59.

Latridius, 59.

Lebasiella, 82.

Lebia, 13.

Leistus, 4.

Lema, 105.

Lembodes, 142.

Lepidophorus, 136.

Leptacinus, 35.

Leptalia, 100 .

LEPTINID.e, 26.

Leptinillus, 26 .

Leptinus, 26.

Leptolinus, 35.

Leptoschema, 70.

Leptostylus, 102.

Leptotrachelus, 12.

Leptura, 101.
Lepturges, 103.

Leptusa, 32.

Lepyrus, 137.

Lesteva, 42.

Lichnanthe, 89.

Licinus, 11.

Liestes, 49.

Ligyrus, 92.

Limnebius, 24.

Limnichus, 62.

Limnocharis, 24.

Limonius, 69.

Limulodes, 44.

Lina, 110.

Liodema, 121.

Liodes, 27.

Liopus, 102.

Liparocephalus, 38.

Lispinus, 43.

Lissonotus, 97.

Lissorhoptrus, 140.

Listotrophus, 33.

Listrochelus, 92.

Listronotus, 137.

Listrus, 80.

Litargus, 53.

Lithocharis, 38.

Litochrus, 46 .

Lixellus, 139.

Lixus, 138.

Loberus, 52.

Lobetus, 79.

Lomechusa, 31.

Longitarsus, 112.

Lophalophus, 136.

Lopheros, 76.

Lophoglossus, 8.

Loricera, 4.

Loxandrus, 10.

Loxopeza, 13.

LUCANIDE, 86.

Lucanus, 86.

Lueidota, 76.

Indius, 68.

Luperaltica, 112.

Luperus, 110.

Lutrochus, 62.

Lyeaina. 76.

Lycoperdina, 49.

Lyeostomus, 75 .

Lyetus, 85.

Lycus, 75.

Ly gistopterus, 76.

Lymantes, 146.

LYMEXYLD.E. 85.

Lymexylon, 85.

Lymnoum, 5.

Lypsimena, 103.

Ly rosoma, 26.

Machlotes, 51.

Macrancylus, 147. 
Macratria, 128.

Macrobasis, 130.

Macrodactylus, 90 .

Macromerus, 143.

Macronychus, 63 .

Macropogon, 63.

Macrops, 138.

Macrorhoptus, 141.

Macrorhyncolus, 147.

Madarus, 144.

Magdalis, 140 .

Malachide, 79 .

Malachius, 79 .

Malacopterus, 95.

Mallodon, 94.

Malthinus, 79.

Malthodes, 79.

Mannophorus, 98.

Mantura, 113.

Marolia, 124.

Maseochara, 32 .

Mastinocerus, 77.

Mastogenius, 74.

Matheteus, 76 .

Matus, 21.

Mecas, 104.

Mecomycter, 81.

Mecotetartus, 103.

Mecynotarsus, 128.

Mecysmus, 119.

Megaderus, 97.

Megalodacne, 49.

Megalops, 40.

Megalostomis, 106.

Megapenthes, 68 .

Megarthrus, 43.

Megasoma, 93.

Megasternum, 25.

Megetra, 130.

Megilla, 46.

Megobrium, 97.

Melamomphus, 134.

Melanactes, 71 .

Melandrya, 124.

MelandRYIDE, 124.

Melanophila, 73 .

Melanotus, 68.

Melasis, 64.

Meligethes, 58.

Melœ, 130.

MELOId E, 130.

Melyris, 81.

Meracantha, 122.

Merinus, 119.

Meristhus, 65 .

Merium, 95.

Merotemnus, 120 .

Mesites, 147.

Mesomphalia, 114.

Metabletus, 14.

Metachroma, 108.

Metaclisa, 120.
Metacycla, 110.

Metaleptus, 98.

Metamasius, 146.

Metaparia, 109.

Methia, 104.

Metoponcus, 35.

Metrius, 4.

Mezium, 83.

Miarus, 141.

Michthysoma, 102.

Micracis, 148.

Micradus, 43.

Micralcinus, 142.

Micralymma, 43 .

Micridium, 44.

Micrixys, 5.

Microcara, 64.

Microcholus, 145.

Microclytus, 99.

Microcyptus, 39.

Microhyus, 142.

Mierolipus, 80 .

Micromalthus, 85.

Micromastus 142 .

Micropeplus, 44.

Microphotus, 77.

Microrhopala, 113.

Microrrhagus, 65 .

Microscapha, 125.

Microschatia, 117.

Microsporus, 45.

Microtonus, 125 .

Mimetes, 134.

Minyomerus, 133.

Miscodera, 15.

Mitostylus, 136 .

Molochrus, 97.

Monachus, 107.

Monarthrum, 147.

Monilema, 101.

Monocesta, 110.

Monocrepidius, 67.

Monœdus, 60.

Monohammus, 102.

Monommide, 123.

Mononychus, 143.

Monotoma, 61 .

Monoxia, 111.

Mordella, 126.

Mordellide, 126.

Mordellistena, 127.

Morio, 5.

Motschulskium, 44.

Murmidius, 51 .

Myas, 8.

Mycetæa, 49.

Mycetina, 49.

Mycetochares, 123.

M YCETOPHAGID F, 53.

Mycetophagus, 53 .

Mycetoporus, 40 .

Mychocerus, 51 .
Mycotretus, 50.

Mycotrogus, 120.

Mycterus, 125.

Mylacus, 135.

Myllrena, 32 .

Myochrous, 108.

Myodites, 132 .

Myrmecochara, 32 .

Myrmechixenis, 53.

Myrmecoxenus, 53.

Myrmedonia, 31 .

Mysia, 47.

Mystaxis, 124.

Nacerdes, 126.

Næmia, 46.

Nauophyes, 141.

Nanosella, 44.

Nanus, 141.

Narthecius, 52.

Nausibius, 51.

Nebria, 4.

Necrobia, 82.

Necrophilus, 26.

Necrophorus, 26.

Necydalis, 99.

Nematidium, 51.

Nematodes, 65.

Nematoplus, 128.

Nemognatha, 130.

Nemosoma, 60.

Nemotarsus, 13.

Neoclytus, 99.

Nenptochus, 135.

Nephanes, 45.

Nicagus, 89.

Nicobium, 83.

Nicotheus, 30 .

Niptus, 83.

Nitidula, 58.

Nitidulid e, 57.

Nocheles, 134.

Nomaretus, 3.

Nomaspis, 130.

Nomius, 5.

Noserus, 116.

Nosodendron, 61.

Nossidium, 44.

Nothodes, 70.

Nothopus, 16.

Nothorhina, 94.

Nothus, 125.

Notibius, 120.

Notiophilus, 4.

Notolomus, 141.

Notomicrus, 19.

Notoxus, 128.

Nyctobates, 119.

Nyctoporis, 116.

Oberea, 104.

Obrium, 97. 
Ocalea, 31.

Ochodæus, 88.

Ochthebius, 24.

Octotoma, 114.

Ocypus, 33.

Odontrus, 89.

Odontonyx, 63 .

Odontosphindus, 86 .

Odontota, 114.

CEdemerid E, 125.

Edionychis, 111.

Edostethus, 66.

Eme, 95.

Estodes, 70.

Olibrus, 46.

Oligomerus, 83.

Oligota, 32 .

Olisthærus, 40.

Olisthopus, 12.

Uloglyptus, 117.

Olophrum, 43.

Omethes, 77.

Omileus, 136.

Omophron, 2.

Omosita, 58.

Omus, 1.

Oncerus, 89

Oncideres, 103.

Oniticellus, 87.

Unota, 14.

Onthophagus. 87.

Onthophilus, 56.

Onychobaris, 144.

Onychylis, $13 \%$.

Oodes, 16.

Opatrinus, 119.

Opetiopalpus, 82 .

Ophryastes, 134.

Opilus, 81.

Opisthius, 4.

Opsimus, 95.

Orchesia, 125.

Orchestes, 141.

Orimodema, 134.

Orizabus, 93.

Orobanus, 43.

Orochares, 42.

Orphilus, 54.

Orsodachna, 105.

Orsonyx, 90.

Orthaltica, 112.

Orthopeplus, 59.

Orthoverus, 46.

Orthopleura, 82.

Orthoris, 144.

Orthosoma, 94 .

Orthostethus, 68.

Orus, 38.

Osmidus, 96.

Osmoderma, 93.

Osorius, 41.

Ostoma, 61 .
Othius, 35.

ОTHNIIDE, 123.

Othnius, 123.

Otidocephalus, 140.

OTIORHYNCHID $\boldsymbol{A}, 133$.

Otiorhynchus, 134.

Oxacis, 126.

Oxoplus, 98.

Oxycnemus, 58.

Oxygonus, 71.

Oxylæmus, 51 .

Oxyomus, 88 .

Oxypoda, 32 .

Oxyporus, 40 .

Oxytelus, 41 .

Ozognathus, 83.

Pachnæus, 135.

Pachybaris, 145.

Pachybrachys, 107.

Pachylobius, 138.

Pachyonychus, 111.

Pachyplectrus, 88.

Pachyscelus, 75.

Pachyta, 100.

Pachyteles, 5.

Pachytychius, 139.

Pactopus, 72.

Prederus, 39.

Palaminus, 39.

Pallodes, 59.

Panagıeus, 5.

Pandeletjus, 135.

Panscopus, 134.

Paragoges, 141.

Paramecosoma, 53.

Parandra, 94.

Paranomus, 70.

Paraptochus, 135.

Paratenetus, 121.

Paria, 108.

Parnida, 62.

Paromalus, 56.

Pasimachus, 4.

Passalus, 86.

Patrobus, 7.

Pediacus, 52.

Pedilophorus, 61.

Pedinus, 120.

Pelates, 26.

Pelecotoma, 132.

Pelenomus, 144.

Pelidnota, 92.

Pelioptera, 31.

Pelonomus, 62.

Pelophila, 4.

Peltastica, 61.

Peltis, 61 .

Pentagonica, 14.

Pentaphyllus, 122.

Pentaria, 126.

Penthe, 124.
Penthelispa, 51.

Pentilia, 47.

Peplogly ptus. 56.

Perarthrus, 98.

Pericompsus, 7.

Perigona, 12.

Perimegatoma, 54.

Peritaxia, 134.

Peritelopsis, 135.

Perothops, 72.

Perthalycra, 58.

Petalium, 83.

Phacepholis, 135.

Phrdromus, 111.

Phrenocerus, 64.

Phalacrid Æ, 46.

Phalacrus, 46.

Phaleria, 121.

Phanreus, 87.

Phausis, 77.

Phellopsis, 116.

Phengodes, 77.

Phenolia, 58.

Phileurus, 93.

Philhydrus, 24.

Philonthus, 33.

Philophuga, 14.

Philotecnus, 14.

Philotermes, 32.

Philothermus, 51.

Phlegon, 65.

Phloeodes, 116.

Phloonarus, 41.

Phløenemus, 50.

Phlœophagus, 147.

Phloopora, 31.

Phloesinus, 149.

Phlœotribus, 149.

Phlœoxena, 13.

Phobetus, 92.

Phodaga, 132.

Phonicobius, 150 .

Phønonotum, 25.

Photinus, 76.

Photuris, 77.

Phryganophilus, 124.

Phthora, 120.

Phycocotes, 140.

Phyconomus, 61.

Phylethus, 121.

Phyllechthrus, 110.

Phyllobænus, 82.

Phyllobius, 136.

Phyllobrotica, 110.

Phyllodecta, 110.

Phyllotreta, 113.

Phyllotrox, 139.

Phymaphora, 49.

Phymatinus, 134.

Phymatodes, 95.

Phyrdenus, 143.

Physemus, 62. 
Physetoporus, 40.

Physocnemum, 95.

Physonota, 114.

Phytalus, 91.

Phytobius, 144.

Phyton, 97.

Phytonomus, 137.

Phytosus, 32.

Phyxelis, 134.

Piazorhinus, 141.

Piazurus, 143.

Piesmus, 8.

Pjezocorynus, 150.

Pilema, 97.

Pinacodera, 14.

Pinodytes, 26.

Pinophilus, 39.

Piodes, 100.

Piosoma, 16.

Pissodes, 138.

Pityobius, 69.

Pityophagus, 59.

Pityophthorus, 147.

Placonycha, 64.

Placusa, 32.

Plagiodera, 109.

Plagionotus, 98.

Plastocerus, 71 .

Plateros, 76.

Platycerus, 86.

Platycholeus, 26.

Platydema, 121.

Platynus, 11.

Platypsylla, 25.

Platypsyllide, 25.

Platy pus, 147.

Platystethus, 41.

Plectrodera, 102.

Plectrodes, 90.

Plectromerus, 97.

Plectrura, 101.

Plegaderus, 57.

Pleoroma, 89.

Pleotomus, 77.

Pleurophorus, 87.

Pleuropompha, 131.

Plinthodes, 136.

Plinthus, 138.

Plocamus, 145.

Plocetes, 141.

Plochionus, 14.

Ploosoma, 49.

Plusiotis, 92.

Pnigodes, 140.

Poradius. 58.

Podabrus, 77.

Podagrica, 113.

Podapion. 137.

Podolasia, 89.

Pecilobrium. 97.

Poecilonota, 73.

Pogonocherus. 103.
Pogonodaptus, 16.

Pogonus, 7.

Polemius, 78.

Polpochila, 16.

Polycaon, 85.

Polycesta, 74 .

Polyclasis, 76.

Polygraphus, 149.

Polylobus, 32.

Polymœchus. 92.

Polyphylla, 92.

Polypleurus, 119.

Polystoma, 31.

Pomphopœa, 131.

Porenspasta, 130.

Porphyraspis, 114.

Porrhodites, 42.

Prasocuris. 109.

Prateus, 121.

Priacma, 85.

Priocera, 81.

Priognathus, 125.

Prionochreta, 26.

Prionocyphon, fit.

Prionomerus, 141.

Prionus, 94.

Pristonychus, 11.

Pristoscelis, 80.

Probosea, 126.

Procas, 139.

Proctorus, 141.

Promecognathus, 4.

Promecops, 136.

Prometopia, 58.

Prostenus, 122.

Prostomis, 52.

Prothalpia, 124.

Protheca, 84.

Protinus, 43.

Psammodius, 87.

Psammocils, 52.

Pselaphid f, 28 .

Pselaphus, 29.

Pselaptus, 29.

Psenocerus, 102.

Psephenus, 62.

Pseudebæus, 80.

Pseudobaris, 144.

Pseudomorpha, 18.

Pseudomus, 142.

Pseudopsis, 40.

Psiloptera, 72.

Psilopyga, 58.

Psoa, 85.

Psydrus, 5.

Psylliodes, 113.

Psyllobora, 47.

Ptenidium, 44.

Pterocolus, 133.

Pteroloma, 26.

Pteroplatus, 97.

Pterostichus, 8.
Pterotus, 77.

Pteryx, 44.

P'tilinus, 84.

Ptilium, 44.

Ptilodactyla, 63.

Ptinella, 45.

Ptinellodes, 44.

Ptinid fi, 83.

Ptinodes, 83.

Ptinus, 83.

Ptomaphagus, 26.

Ptosima, 74.

Ptychodes, 102.

Purpuricenus, 98.

Pyanisia, 122.

Pycnoglypta, 43.

Pycnomerus, 51.

Pyractomena, 76.

Pyrochoa, 129.

PyrochroId E, 129.

Pyrophorus, 71.

Pyropyga, 76.

Pyrota, 131.

Pyrotrichus, 99.

Prthid a, 125.

Pytho, 125.

Quedius, 33.

Rharalus, 81.

Rhæboscelis, 75.

Rhagium, 100.

Rhagodera, 50.

Rhanis, 49.

Rhantus, 22.

Rhexius, 30 .

Rhigopsis, 134.

Rhinandrus, 119.

Rhinomacer, 133.

RHINOMACERID , 133.

Rhinoncus, 144.

Rhinoplatia, 126.

Rhinoscepsis, 30 .

Rhinosimus, 125.

RHIPICERID A, 64.

Rhipidandrus, 86.

RHIPIPHORIDE, 132.

Rhipiphorus, 132.

Rhizophagus, 59.

Rhodobænus, 146.

Rhombodera, 14.

Rhopalnphora, 97.

Rhopalopleurus, 149.

Rhopalopus, 95.

Rhoptobaris, 144.

Rhyncheros, 75.

RHYNChitide, 133.

Rhynchites, 133.

Rhynchophorus, 146.

Rhyncolus, 147.

Rhypobius, 46.

Rhypodes, 135. 
Rhyssematus, 142.

Rhyssemus, 87.

Rhyssodes, 51 .

RHYSSODIDE. 51.

Rhytidosomus, 144.

Romaleum, 96.

Rosalia, 97.

Rugilus, 38.

Rutela, 92.

Sacium, 46.

Salpingus, 125.

Sandalus, 64.

Saperda, 104.

Saprinus, 56.

Sarpedon, 65.

Saxinis, 106.

Sealenarthrus, 30 .

Scalidia, 51.

Scaphidema, 121.

ScaplididD A 45.

Scaphidium, 45.

Seaphinus, 94.

Scaphisoma, 45.

Scaphium, 45.

Scaptolenus, 72.

Scarabeide, 86.

Scarites, 4.

Scelolyperus, 110.

Schizax, 98.

Schizillus, $11 \%$.

Schizogenius, 5.

Schizophilus, 65 .

Schizopus, 74.

Schizotus, 129.

Schœnicus, 116.

Scierus, 149.

Sciopithes, 134.

Scirtes, 64.

Scolytide, 147.

Scolytus, 148 .

Scopreus, 38.

Scotobates, 119.

Seotochroa, 124.

Scotodes, 125 .

Scraptia, 125.

Scutopterus. 22.

SCYDMANID A, 28.

Scydmænus, 28.

Seymnus, 48.

Sevphophorus, 146.

Scythropus, 136.

Selenophorus, 17.

Serica, 90.

Sericoderus, 46.

Sericosomus, 70.

Serropalpus, 124.

Siagonium, 44.

Sibynes, 141.

Sicyobius, 103.

Silis, 78 .

Silpha, 26.
Silphid.e, 26.

Silusa, 32.

Silvanus, 51.

Simplocaria, 61.

Sinodendron. 86 .

Sinoxylon, 84.

Sitodrepa, 83.

Sitones, 136.

Sitophagus, 119.

Smierips, 59.

Smieronyx, 139.

Sinierus, 45.

Smodicum, 95.

Soronia, 58.

Sosylus, 51.

Spalacopsis, 104.

Spathizus, 77.

Spermophagus, 114.

Sphrenothecus, 98.

Sphæridium, 25.

SPHARIIDE, 45.

Sphrerites, 26.

Sphærius, 45.

Sphæroderma, 113.

Sphæromorphus, 89.

Sphalma, 125.

Sphenophorus, 146.

Sphenostethus, 94.

SPIIndid.E, 86.

Sphindus, 86 .

Spilotus, 124.

SPONDYLID E, 94.

Spondylis, 94 .

Spongopus, 18.

StapHYlinid.£, 31 .

Staphylinus, 33.

Statira, 123.

Stelidota, 58.

Stenaspis, 97.

Stenelmis, 62.

Stenispa, 114.

Stenochidus, 122.

Stenocolus, 63.

Stenodontes, 94.

Stenolophus, 17.

Stenomimus, 147.

Stenomorphus, 16.

Stenopelmus, 139.

Stenopodius, 114.

Stenoscelis, 147.

Stenosphenus, 98.

Stenotarsus, 49.

Stenotrachelus, 125.

Stenotricus, 119.

Stenus, 35.

Stenusa, 32.

Stephanocleonus, 138.

Stephostethus, 59.

Stereopalpus, 128.

Stethobaris, 145.

Stethon, 64.

Stibia, 115 .
| Stictocranius, 37.

Stilicopsis, 39.

Stilicus, 38.

Sitolonis, 10

Strangalia, 100 .

Strategus,' 93.

Strigoderma, 92.

Stromatiunn, 96.

Strongylium, 122.

STYLOPID.E, 132.

Stylops, 132.

Styloxus, 104.

Sunius, 39.

Suphis, 19.

Symbiotes, 49.

Symphora, 124.

Synaphota, 102.

Syncalypta, 62 .

Synchita, 50.

Synchroa, 124.

Syneta, 105.

Syntomium, 42.

Systena, 112.

Tachinus, 39.

Tachycellus, 18.

Tachygonus, 143.

Tachy porus, 39 .

Tachypus, 5.

Tachys, 7.

Tachyusa, 31.

Tanaops, 79.

Tanarthrus, 129.

Tanygnathus, 32 .

Tanymecus, 135.

Tanyrhinus, 42.

Tanysphyrus, 139 .

Taphrocerus, 75 .

Taphroscelidia, 51.

Taricanus, 103.

Tarsustenus, 81.

Tecnophilus, 14.

Tegrodera, 132.

Telephanus, 52 .

Telephorus, 78.

Telmatophilus, 52 .

Temnopsophus, 79 .

Tenaspis, 76 .

Tenebrio, 119.

Tenebrioides, 60.

Tenebrionellus, 119.

TFNeBRIONIDE, 115.

Teretriosoma, 57.

Teretrius, 57.

Tetracha, 1.

Tetragonoderus, 13.

Tetraonyx, 131.

Tetraopes, 104.

Tetrapriocera, 85 .

Tetratoma, 124.

Tetropium, 95.

Tetrops, 104. 
Thalpius, 13.

Thalycra, 58 .

Thanasimus, 82 .

Thaneroclerus, 82 .

Tharops, 64.

Tharsus, 121.

Theea, 84.

Thecesternus, 133.

Thermonectes, 22 .

Thesium, 30 .

Thia, 104.

Thiasophila, 32.

Thinobius, 42.

Thinopinus, 33 .

Thinoxenus, 135 .

Thricolema, 105.

Thricolepis, 135.

Thricomigus, 134.

Thrincopyge, 74.

ТнRoscid $2,72$.

Throscinus, 62 .

Throseus, 72 .

Thyce, 92.

Thymalus, 61.

Thysanocnemis, 141.

Thysanœs, 148.

Tilea, 42.

Tillomorpha, 99.

Tillus, 81.

Timarcha, 109.

Tisiphone, 59.

Tmesiphorus, 29.

Tomarus, 52 .

Tomicus, 148.

Tomoderus, 128.

Tomoxia, 126.

Toposcopus, 132.

Toxidium, 45.

Toxonotus, 150.

Toxotropis, 150 .

Toxotus, 100.

Trachodes, 140.

Trachykele, 73.

Trachypachys, 2.

Trachy phlous, 135.

Trachyscelis, 121.

Tragidion, 98.

Tragosoma, 94.

Trechicus, 12.

Treehus, 8.

Triachus, 108.

Triarthron, 27.

Tribalister, 55.

Tribalus, 55 .

Tribolium, 120.

Trichalophus, 136.

Trichischius, 146.
Trichius, 94.

Trichobaris, 144.

Trichodes, 81.

Trichodesma, 83.

Trichonyx, 30 .

Trichopsenius, 39 .

TRICHOPTERYGIDE， 44 .

Trichopteryx, 44.

Trichotheca, 108.

Trichoxys, 98.

Trierania, 130.

Tricrepidius, 67.

Triga, 44.

Triglyphus, 136.

Trigonodemus, 42.

Trigonogenius, 83 .

Trigonophorus, 33 .

Trigonoseuta, 134 .

Trigonurus, 44.

Trimium, 30 .

Trimytis, 115.

Triorophus, 115.

Triphalus, 115.

Triphyllus, 53 .

Triplax, 50.

Trirhabda, 110.

Tritoma, 50.

Trogloderus, 118.

Trogodendron, 81.

Trogoderma, 54.

Trogophlœus, 41.

Trogosita, 60 .

Trogositide, 60 .

Trogoxylon, 85.

Trophimus, 79

Tropideres, 150.

Tropisternus, 24.

Trox, 89.

Trypherus, 79.

Trypopitys, 83.

Tychius, 141.

Tychus, 29.

Tyloderma, 142 .

Tylonotus, 96.

Tylopterus, 141.

Tylosis, 98.

Tymnes, 108.

Tympanophorus, 33 .

Tyndaris, 74 .

Typhoea, 53.

Typocerus, 100.

Tyrus, 29.

Tytthonyx, 77 .

Ulkeus, 55.

Ulochretes, 99.
Uloma, 121.

Ulosonia, 120.

Ulus, 120.

Upis, 119.

Urodera, 106.

Urographis, 103.

Usechus, 116.

Valgus, 94.

Vrilletta, 84.

Wollastonia, 147.

Xanthochroa, 126

Xantholinus, 35.

Xanthonia, 108.

Xanthopygus, 33.

Xenistusa, 39.

Xenomycetes, 49.

Xenorchestes, 150.

Xenorhipis, 73.

Xenos, 132.

Xestobium, 83.

Xestonotus, 18.

Xyleborus, 148.

Xyletinus, 84.

Xylita, 124.

Xylobius, 65.

Xylocleptes, 148.

Xylocrius, 95.

$\mathrm{X}$ ylophilus, 128.

Xylopinus, 119.

Xyloryctes, 93.

Xylosteus, 100.

Xyloterus, 148.

Xylotrechus, 99.

Yuccaborus, 146.

Zabrotes, 115.

Zacotus, 15.

Zaglyptus, 142.

Zagymnus, 99.

Zalobius, 42.

Zamodes, 96.

Zaplous, 103.

Zarhipis, 77.

Zascelis, 143.

Zenoa, 64 .

Zeugophora, 105.

Zilora, 124.

Zonitis, 130.

Zopherus, 116.

Zuphium, 12.

Zygobaris, 145.

Zygops, 143. 


SMITHSONIAN INSTITUTION LIBRARIES 